\title{
U. S. NATIONAL MUSEUM
}

\section{BULLETN}

NO. 40

1892

SMITHSONIAN

I.IBRARY 


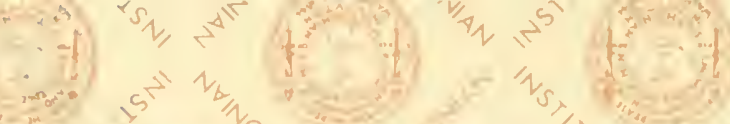

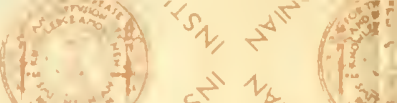

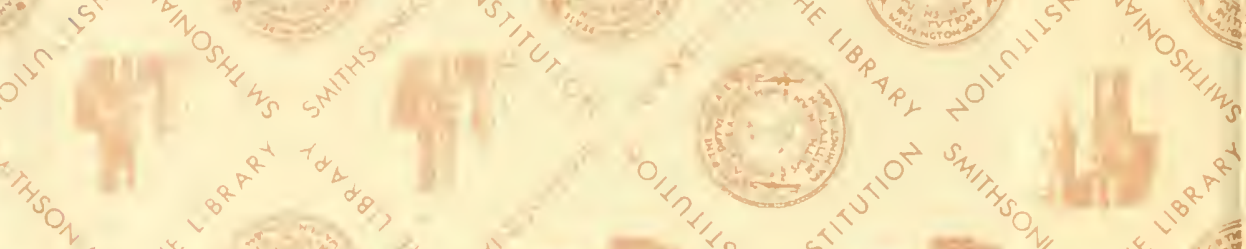

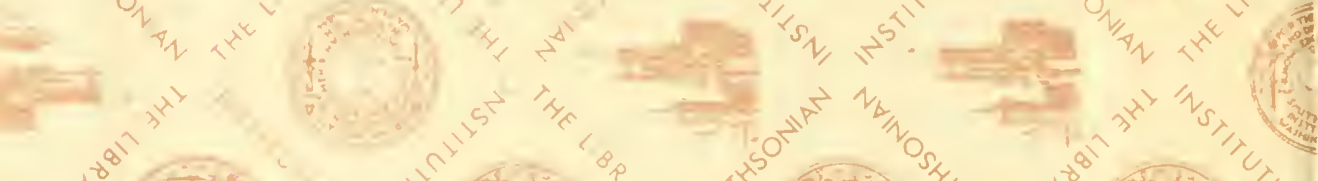

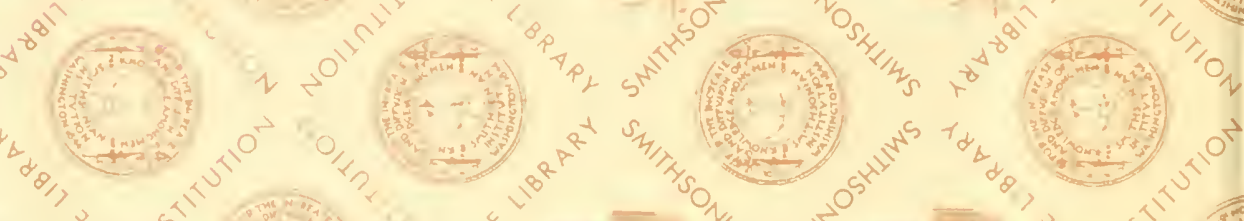

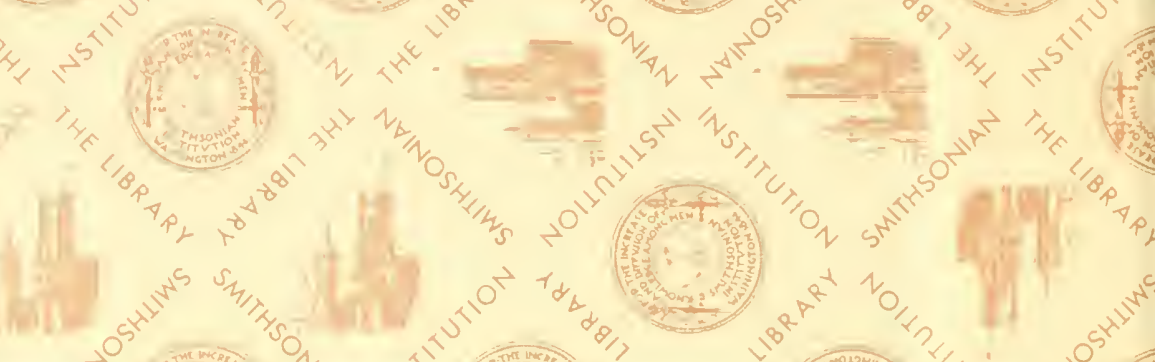

at

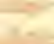

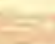

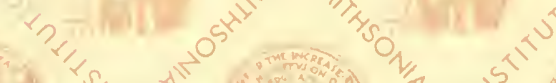

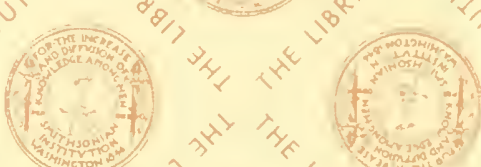<smiles>[CH]C</smiles><smiles></smiles>

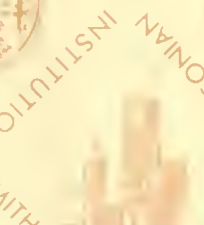

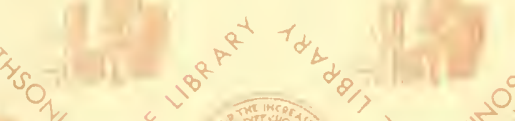
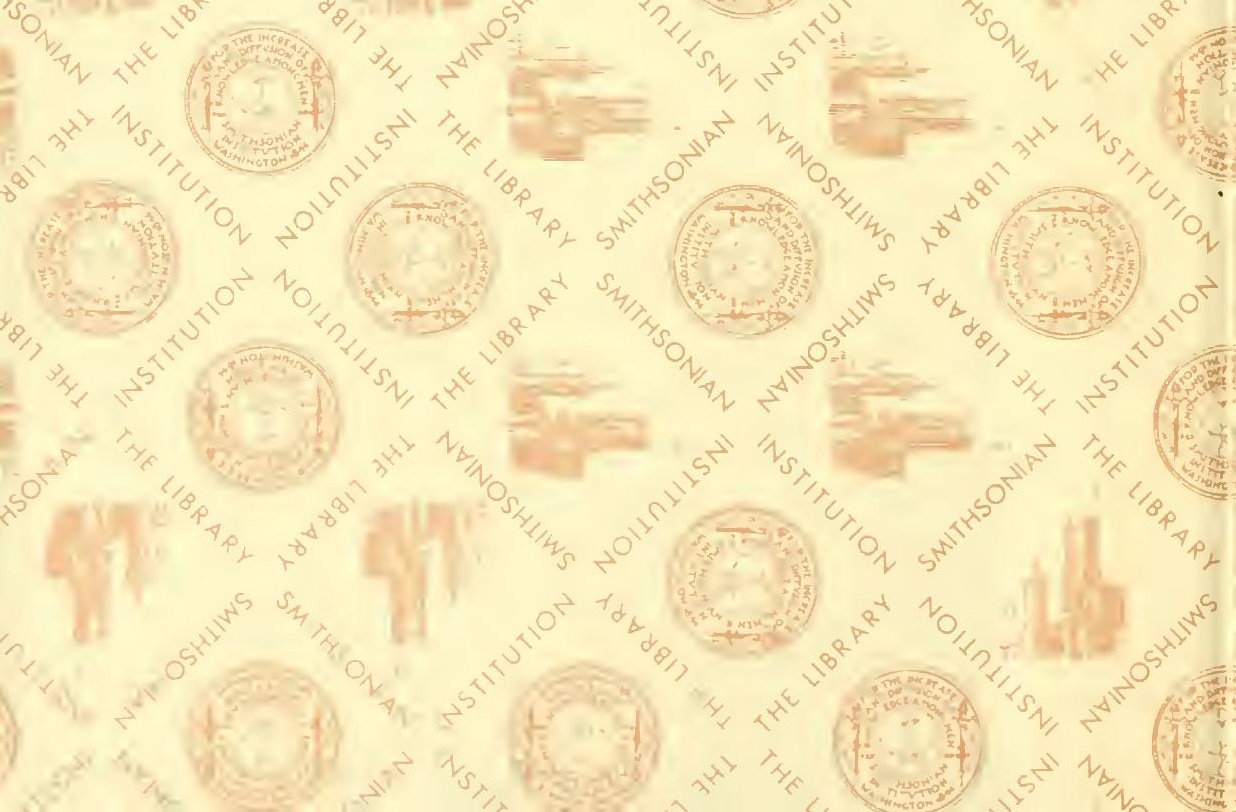





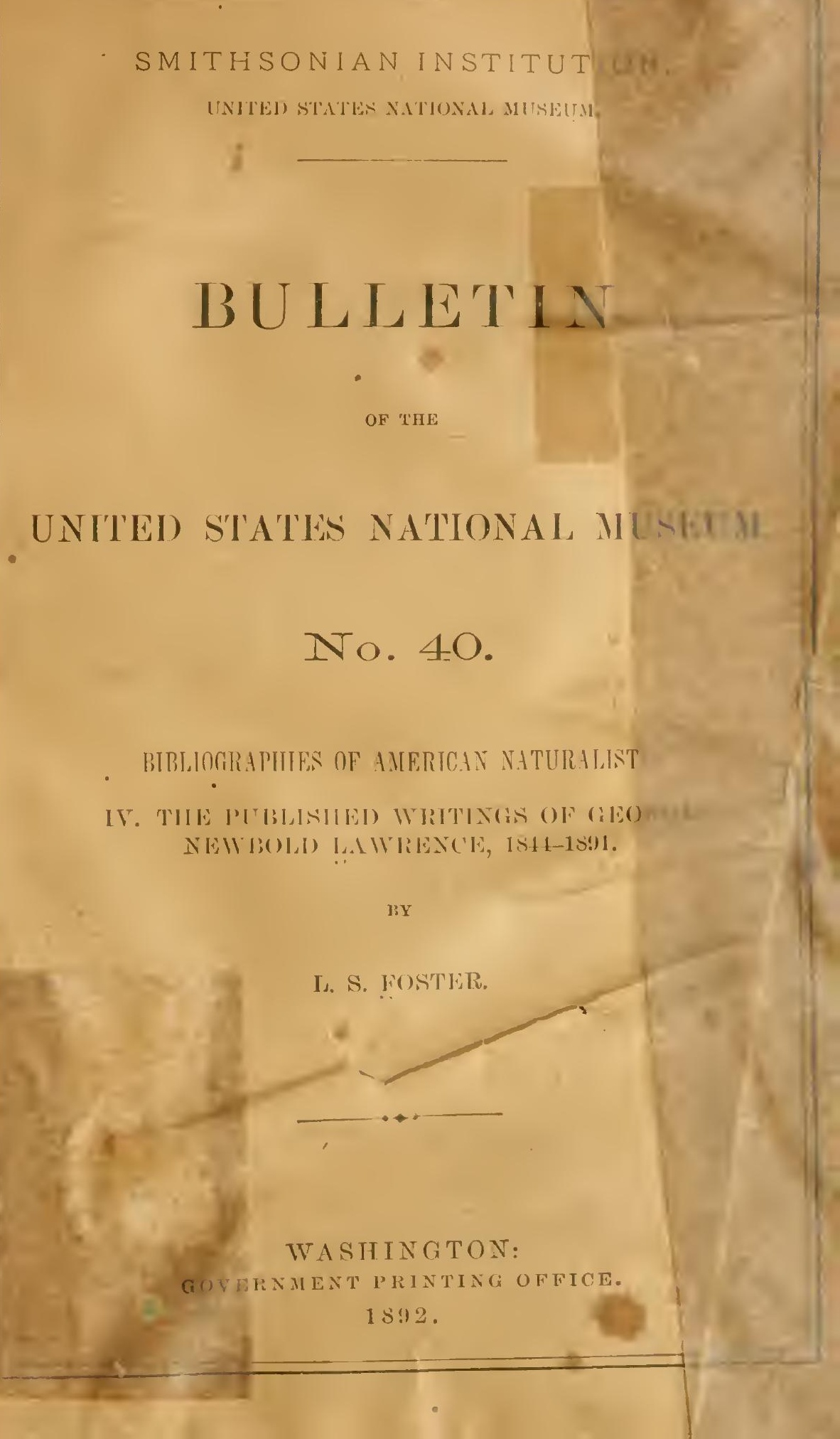


$4=$

$1+\frac{1}{2}=$

$\operatorname{lig}$

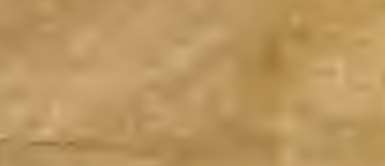

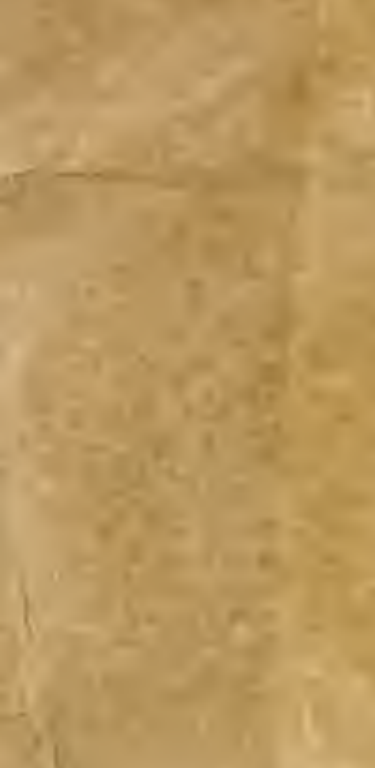

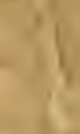

$4=$

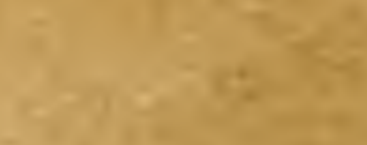

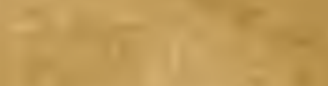

$=$

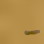

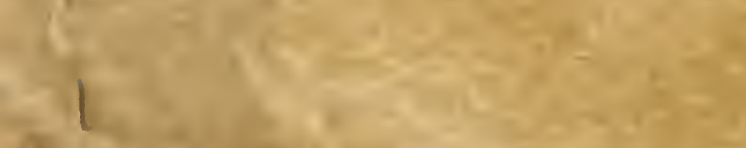

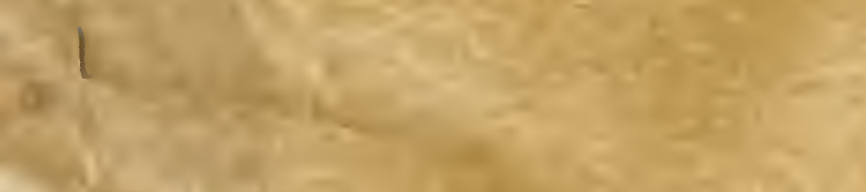

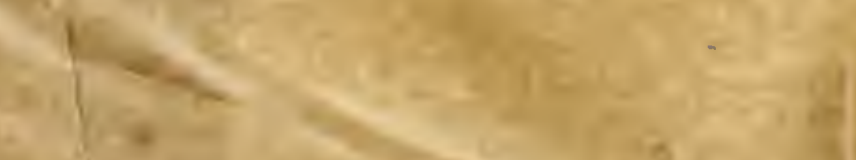

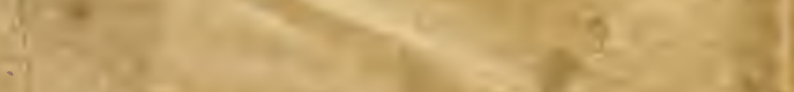

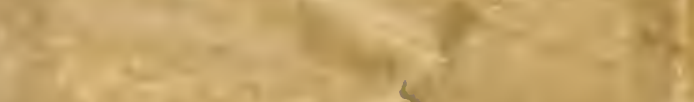

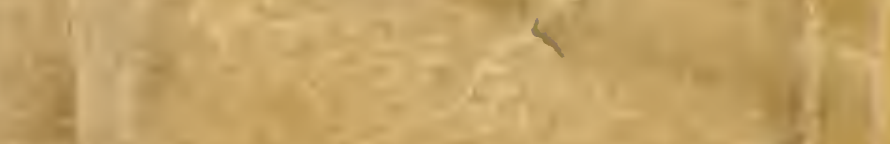

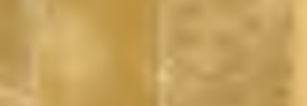

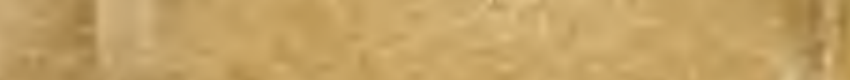

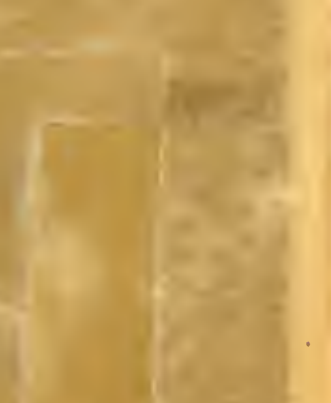

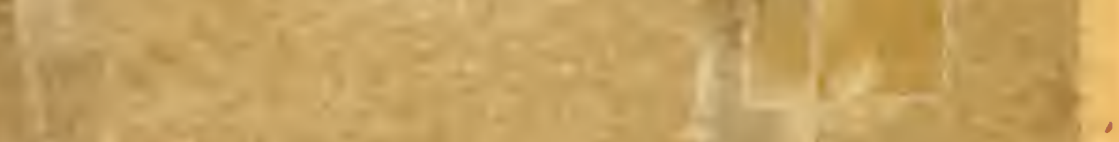

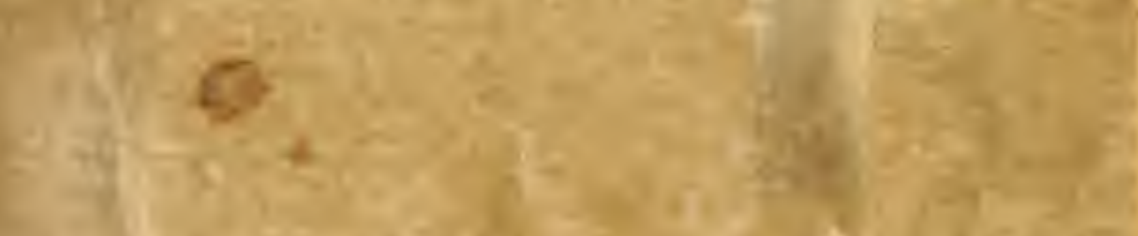

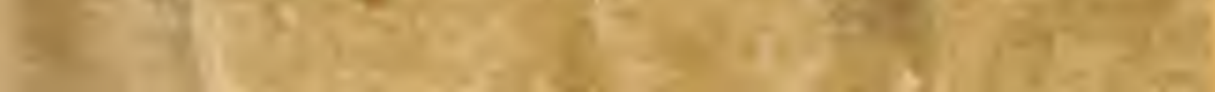


BRDSMITHSONIAN INSTITUTION, UNITED STAES NATIONA, MUSEUM.

\section{BULLETIN}

of $\mathrm{rtw}$

\section{UNITED STATES NATIONAL MUSEUM.}

\section{No. 10.}

BIBLIOGRAPHIES OF ANERICAN NATURALISTS:

IV. THE IUUBLSHED WRITINGS OF GEORGE NEWBOLI) LAWRENCE, 1St4-1S!1.

L. S. FOSTER.

WASHINGTON:

GOVERNMENT PRINTING OFFICE. 


\section{AIVERTISEIENT.}

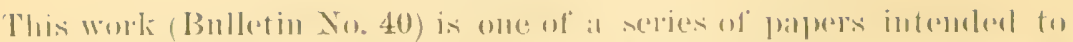

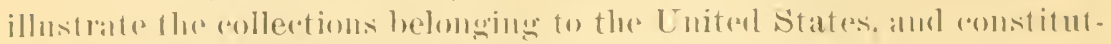
ing the National Musemu, of which the sumithsmian Institution was

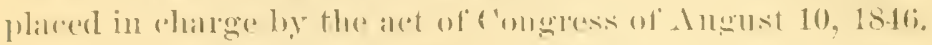

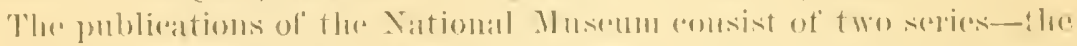

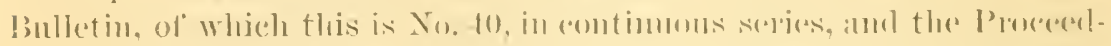
ings. of which the fifternth volume is now in press. A small edirion

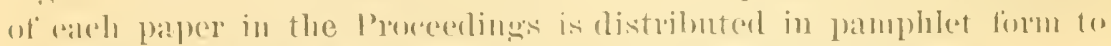
sereialists in alvance of the pulblieation of the homul volume.

T'he Bulletiu of the Sational Musemm, the pulbliention of whirele was (4)

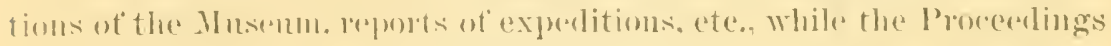

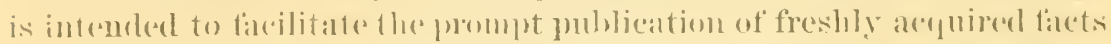

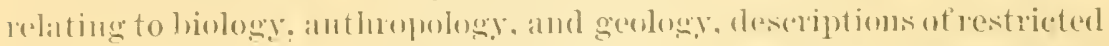

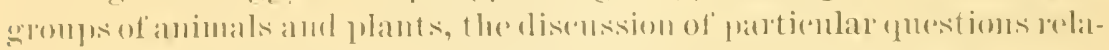

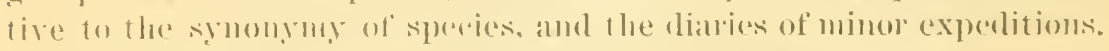

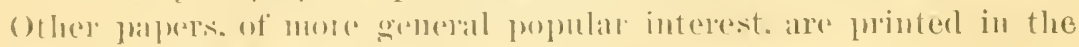
Appendix to the Ammual liepurt.

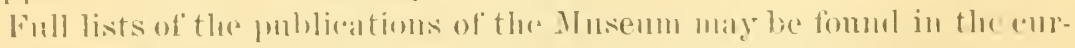

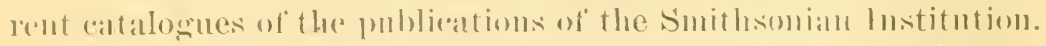

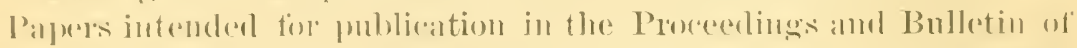

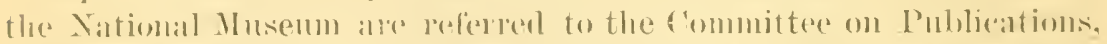

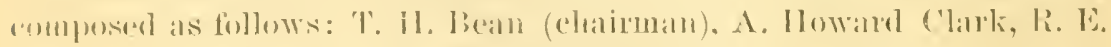

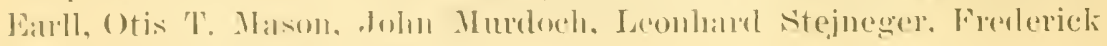
II. Jrine, and lester li. Mark.

S. P. LANGLEL,

siceretery of the simithsoniun Institution.

11.111

II 


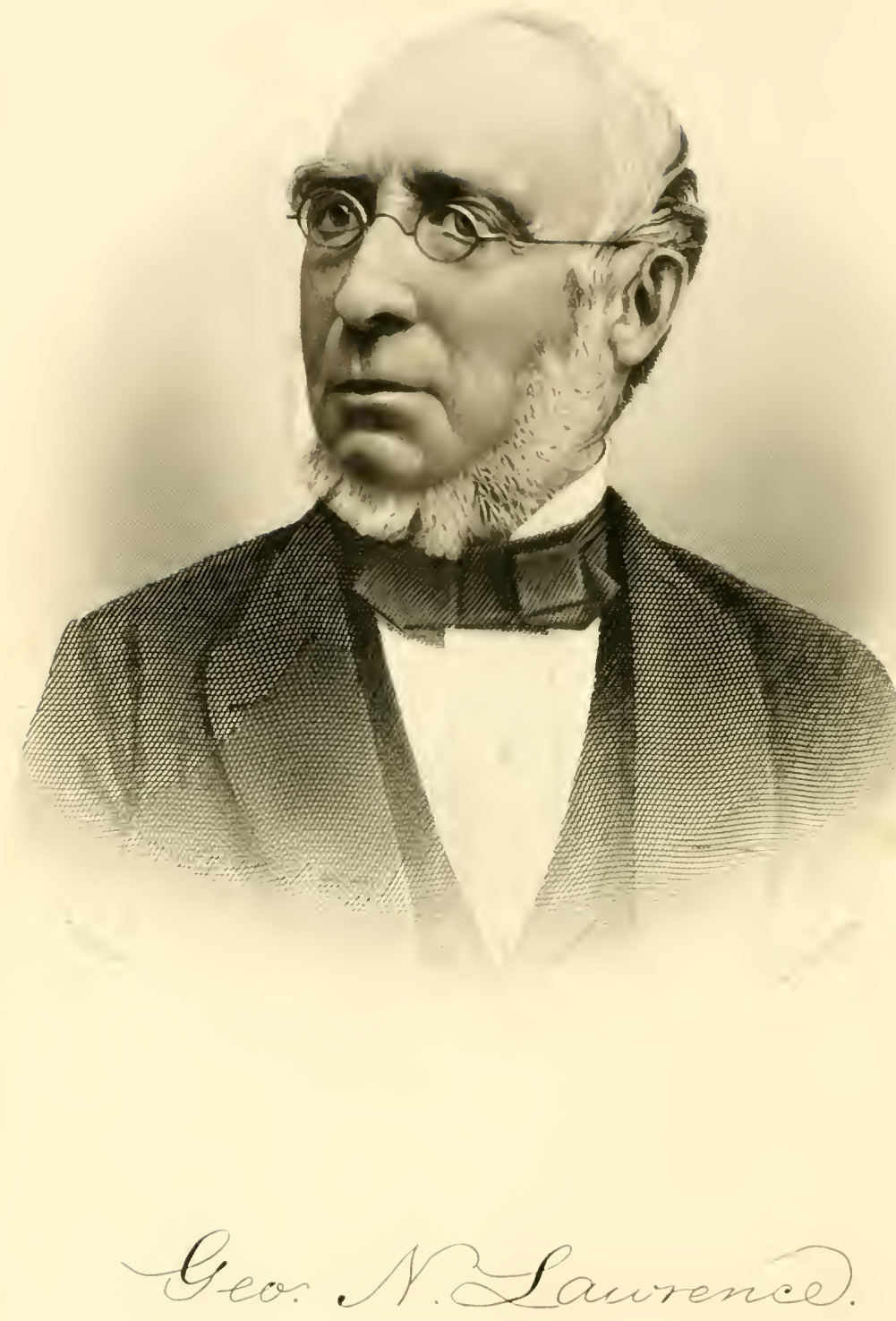


BHBLOGRAPHIES OF AUERICAN NATURALISTS

$$
\text { I V. }
$$

\section{THE PUBLISHED WRITINGS}

\section{GEORGE NEWBOLD LAWRENCL, 1844-1891.}

L. S. FOSTER.

WASIIINGTON:

GOVERNMENT PRINTING OFFICH.

1892 . 



\section{CONTE N TS.}

Frontispiece, portrait of Mr. (ieorge N. Lawrence.

I'ape.

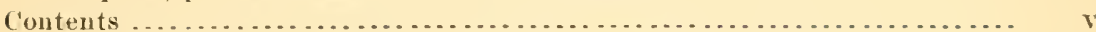

Biographical sketrh ...................................... vil

Avian genus named in honor of Mr. George N. Lawrene .............. IX

Species of hirls named in honor of Mr. George $\mathrm{X}$. Lawrence........... IX

Chronological catalogne of the publications of Mr. George N. Lawrence, 1844 to 1891

Alphabetical list of new sprecies and subspecies of birts, deseribed ly $\mathrm{Mr}$.

Grorge $\mathrm{N}$. Lawrence, 1846 to 1891 , with habitat of type sperimen........ 101

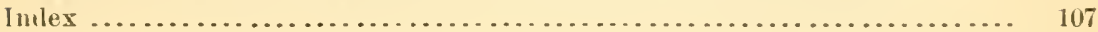





\section{BIOGRAPIICAL, SKETCH OF GBORGE NEWBOLD LAIIRENCLE.}

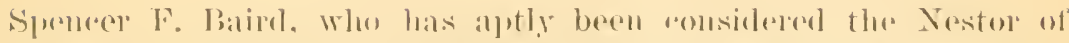

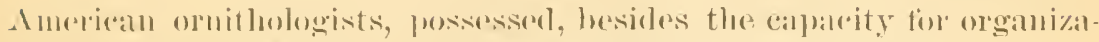

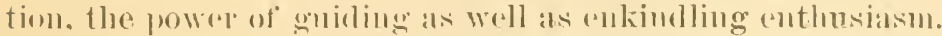

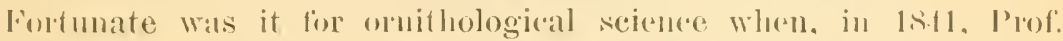

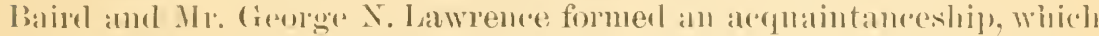
smon ripeneel into a close and lasting intimacy. Stimulated by this.

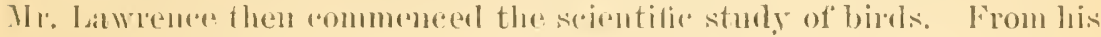

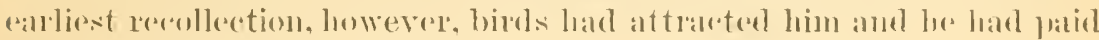
a)siclerable attrition to them.

George Newbold Lawrence was born in the reity of New York, where

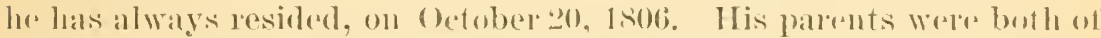

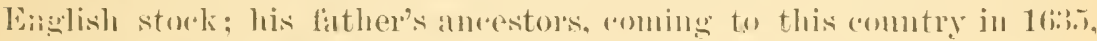
fouslly settled in New Furk, and those on his mother's sicle lonated in liullington Comnty, N. J., in rosit.

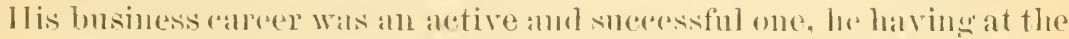
early age of twenty anterod into a latinel'slips, with his father and others, in the wholusald dlug business in Now Yorli City. In this lae ("ontinued for thirty-six years, devoting assioluonsly his spare time fo

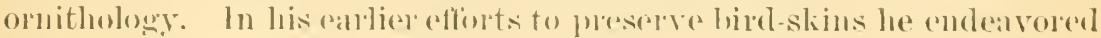

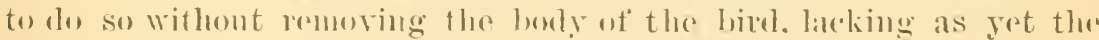
commsel of a slibled taxidemist. This indiontes that he folt an ine-

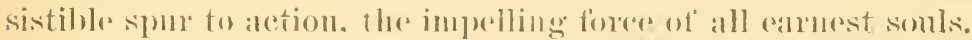

liepratedly he was of selvire to the sulthsonian lustitution in the letails of onthtting expeslitions for ficld work, and in many ways aided

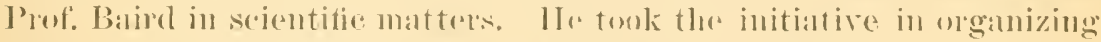
the Ober experlition to the lasiser Antilles.

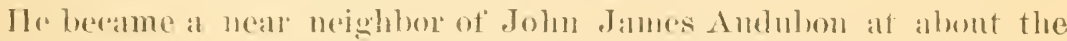
close of the lite of that illustrims matmalist. amel was very lambliar

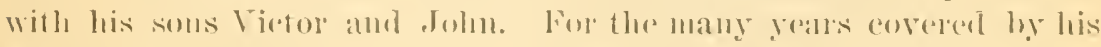

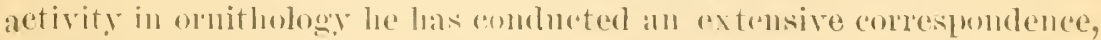

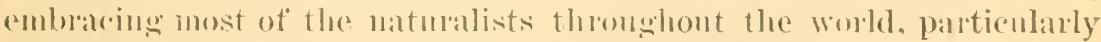
those interesterl in olıitholong.

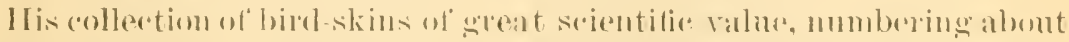

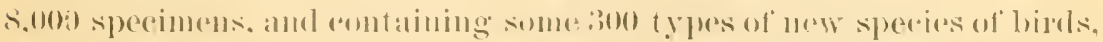

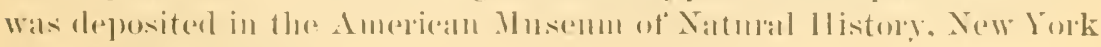
City, in Maly, 1sist. 


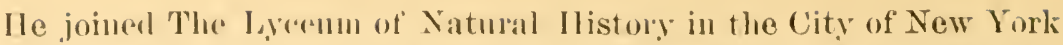
in 1st.t, aml mw is a member, a fellow, and a batron of its surcessor, The Now York Arademy of Soienres. Upon the organization of 'The American Onithologists' Union, in 1sis, lıe breame an active member, and at Its biohth Congress, November, 1890, lie was eleeted an honorary member. (of 'The Limanan Sorety of New York, ho likewise is an homorary member, having been chosen as such April $1: 3,18 T 8$. The liritish Ornithologists Union made him a foreign member in 157:, and lie is also eonnected with a large mumbor of kindrod sorietios in this and other comuties.

Thromghout his soientifir carex Mr. Lawence has ammanded the confidence and respert of all of his rontemporandes, with many of whom he las formed rorlial fiemdships, and it is with amazement that we "onsider the long list of great mames, fom Viablot to thr present time, that since the birth of Mr. Lawence have inporessed indelihly the brilliancy of their splendirl genius on Amerioan ornithology. Wilson, Andubon, Bonapartr, Brewer. Nuttall, Baird, Cassin, Girand, De Kay, and mamy moro have eome, and passed on, during the streteh of one life. Of a verity American ormithological soience has been favored in its leadris doring the last fourscole years.

Tha beneficial influence of the lahors of' Mr. Lawence, with pen and pencil, on the progless of Anerin'an ornithology has heen gleat and undisputed, but it was particularly amoug the avifauna of the West Indies, Mexiro, Central and somtl Amorica that his most stremums efforts were exerted. All but 17 of the 31 ! new spories of birls describud ly him eame fiom that region. Endowed with great power of analysis and a remarkable patience, lie applied these to the investigation of speeific distinctions, and his conclusions have but rarely been questioned.

A scrutiny of the titles of his numeroms papers will finlly indieate the wide scope and excellent character of the work of this veteran ornithologist.

1. N. F.

New York City, A "umst 1, 18.91. 
AVIAN GENUS NAMED IN HONOR OF MR. GEORGE N. LAWRENCE.

Laurenciu, Riolgway. Deseription of a new gems of Tyrannida fiom Sauto Domingo. By Robert Rirlgway. <The Auk. Vol. III, No.:3,

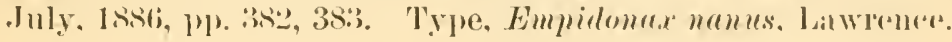

SPECIES OF BIRDS NAMED IN HONOR OF MR. GEORGE N. LAWRENCE, ARRANGED IN ORDER OF PUBLICATION, WITH BIBLIOGRAPHICAL DATA AND HABITAT.

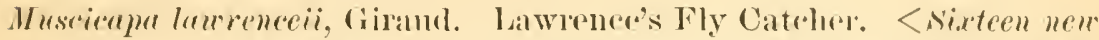
spectes of Texos birds. By lacol, P. Girand. jir. Now Tork, 1841. p. !. Pl. I, Fiฏ. ‥ Ilabitat, Texas.

This species is now Myluerche laurenceii ((iIr.), No. [455] of the A. (1) U. Check-list of Xorth American Pirds.

Carduelis luereneci, Cassin. Lawreners's (ioldtinel. Deseriptions of new speries of Birds of the Genera l'arns, Linn.; Emberiza, Limm.; Carduclis, Briss.; Myothera, Ill.; and Lemonerpes, Sw., specimens of which are in the collection of the Academy of Natural siriences of Philadelphia. By Jolun Cassin. <Proce Acad. Nut. sci. Philu., Vol. r, Oetober, 1s,o), 1. 10.5, Pl. v. Habitat, Sonomal and sau l)iego, California.

Now spinus lawencei (CAss.), No. 5331 of A. O. T. Cherk-list.

Fregettr laserencii, Bonaparte. The Black and White Stormy Petrel. <Cons. Gen. Arimm, C. L. Bonaparte, Vol. Ir, 18.r., p. 198. I Ialoitat, Florida.

Cyphorinus leurencii, Selater \& Salvin. Lawrence's Wren. Notes on a collection of birck fiom the Isthmms of P'anama. By l'. L. Selater

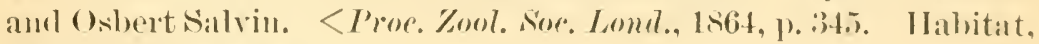
Veragua, P’anamá.

A colored plate of this bird may lue fomnd in Exotic Omitlology. Selater of Silvin. London, 1869 .

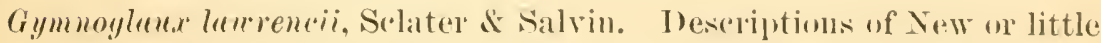
known Amerien lirds of the Families Fringillida. Oxyrlamphidat, Bucconidate and Strigida. By P. L. Schater and Osbert Salvin.

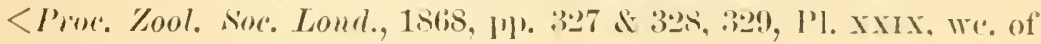

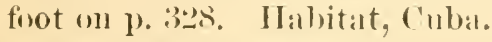

Helminthophreya lanerenii, Herrick. Lawrences Warbler. Description of a new species of Hehuinthophaga. By Harold Herrick. < r'roe.

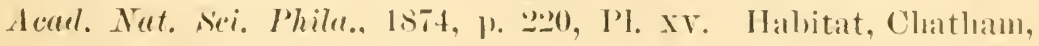
New Jersey.

Now Helminthophila lanemed (Honuck), No. 20 of A. (1. U. Hypothetical list. Considered al hybrisl. 


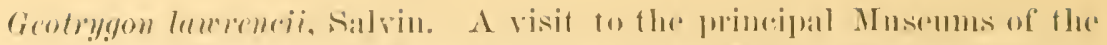
United states, with Notes on sume of the Birds rontained therein.

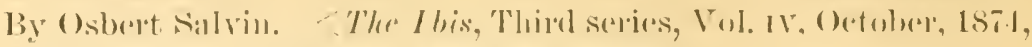

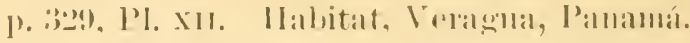

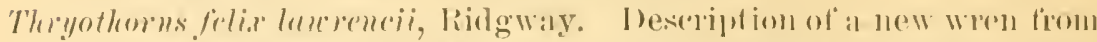

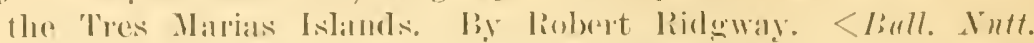

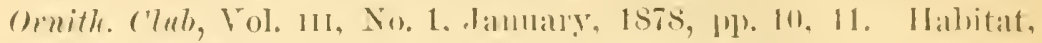

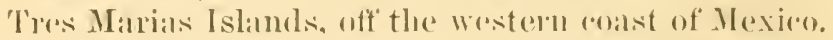

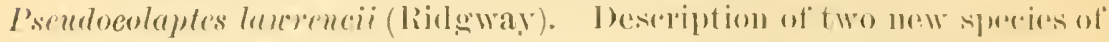
lirels from Costa Rica, and motes on other lare sjecios firm that

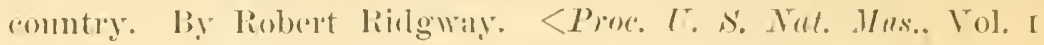

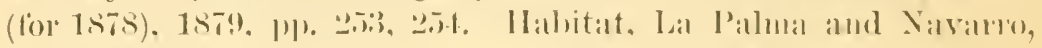
Costa Rirail.

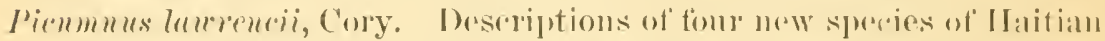

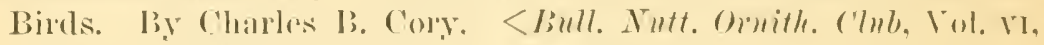

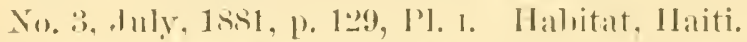

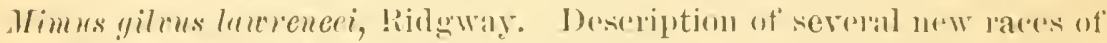

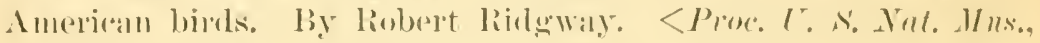

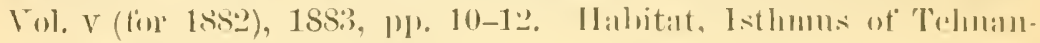

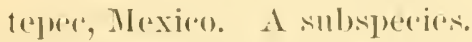

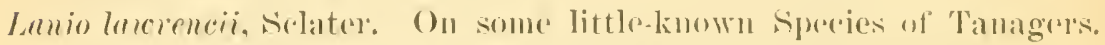

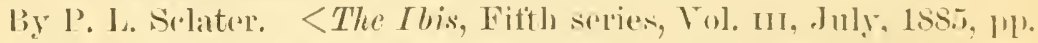

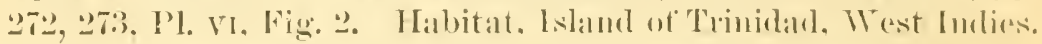

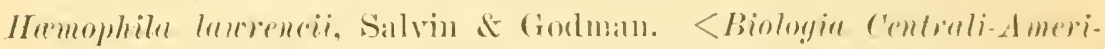
emm, Vol. 1, 187!-1857, 1\% 397. 'This signature issmol August. 1886. Ilabitat, Juchitan, near 'Tehumutepere, Mexico.

Ieterus lurvencii, Cory. Descriptions of six supposed new spores of

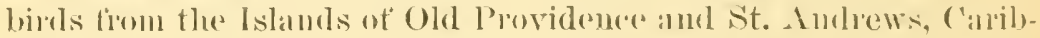

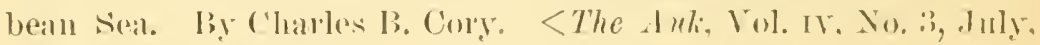

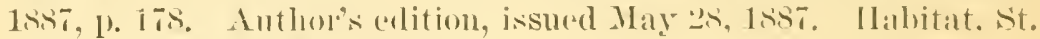
Androws Island, iVrast Indies.

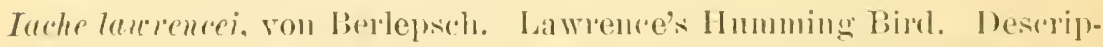
tions of mew sumedes and Subsperies of 'Trorhilida. liy llams vom

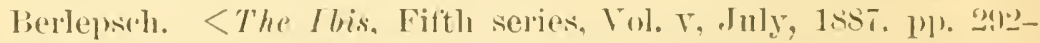

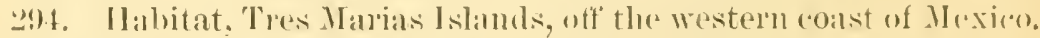

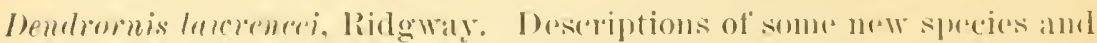

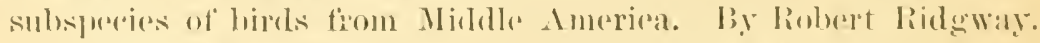

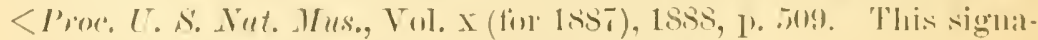
tmre issmorl dugnst ti, 18Ss. Ilalitat. Panamí.

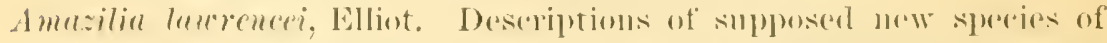

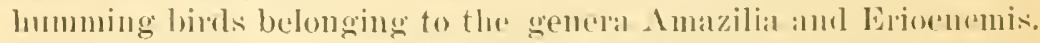

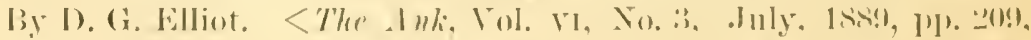

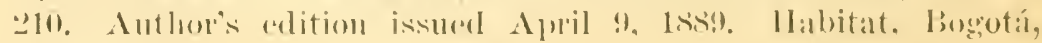
(olombia. 
Empidonax lanrencei, Allen. Deseriptions of New speries of Sonth Amerien Birds, with remanks on varions of her littleknown Speries.

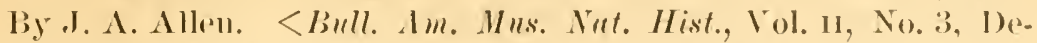

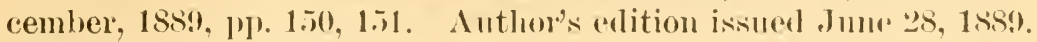
Habitat, South America.

sclermus larrencei, lidgway. A review of the genus Sclermus of

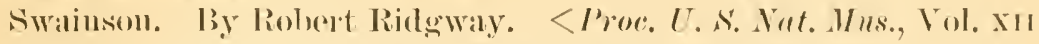
(for lss!), 1890, 11). 2!), :30. This article, No. 762, was publishe+l Fehruary 5, 18!0( Ilabitat, Bahia, Brazil (?) 



\section{BIBLIOGRAPHY OF' 'THE PUBLISHED WRITINGS}

\section{(1) \\ GEORGE NENBOLD LAWRENCE, 1844-1891.}

\section{CHRONOLOGICAL CATALOGUE.}

1.

1814. Lawrexce, fienkcis $\mathrm{N}$. Letter dated New York, Deetmber 3, 1812, to J. P. Girnud, jr. <The Birds of Long Island. By .J. P. Girand, jr. New Yurk, 1814, 1. 309 .

1. A nas penelope .......................... Long Island, X. I page 309

\section{2.}

1816. Lawricace, George $N$. Deseription of a New Speeies of Anser. By George X. Lawrenee, Read March 16th, 1816. LAmu. Lye. Nat. IFist. New York. Vol. IV, 1817, pp. 171, 172, plate No. xil of Anser nigricans. Issued in Nos. 6, 7 , August, $1 \times 46$.

1. Anser nigrirans, n. s..................Egg Harbor, N. J...........pages 171, 172

3.

1847. LAWRECE, GEonge N. Extract from the Minutes of the Lyeeum of Natural History of New York, February 8th, 1847. Mr. Lawrence read a descruption of at new species of Procellaria from Florida, which he proposes to eall Brevirostris. <Aun. Lye. Tat. Hist. Tew York. Vol. IV, 18t7, third page of cover of Nos. 8, 9, Aluil, 1817.

1. Procellaria bervirostris, n. s...... Florida. Third page of corer.

z.

1847. Lawrexce, Georie $N$. Description of a New Species of Procellaria. By George N. Lawrence. Real Fobruary 18th, 1817. <Aun. Lyc. Tat. Hist. New Fork. Vol. IV. 18.17, pp. 475,476, plate No. $\mathrm{x}$ of Procellaria meridionalis, Lawrence. Issued in Kos. 10, I1, July, 1847.

1. Procellaria meritionalis, п.. pages 475,476

\section{5.}

1819. LAWRACE, GEORGE X. Observations on the preeeding Paper (one by John Bell, "Observations on th" Limosa Scolopacea of say"). By George $x$. Lawrenee. Rad January 7 , 1849. <Ann. Lye. Nat. Hist. Ner Tork. Vol. v, 1852, pp. 4.5, plate No. I of Macrorhamphus scolopaceons, Latwr., \& in summer and $\rho$ in winter. Issued in No. 1, May, 1819.

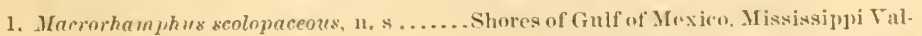
ley and westward ............... pages 4,5

Bull. 40, N. M. - 1 
6.

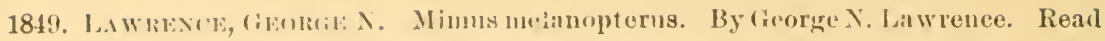
16th April, 1819. <.tun. Lyr. Nat. Hist. New Fork. Vol. v, 1852, 11. 35, 36,

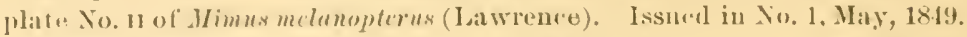

1. Mimns melanopterus, 1. s ............ Veneznela.................. pages 35, 36

\section{7.}

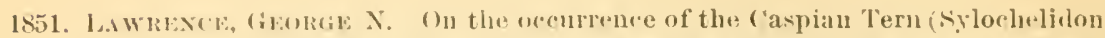
('ispins) in North Americal. By Georgr X. Lawrence. Read Jaỵ bith, 1850. <inn. Lyc. Nat. Hist. New Jork. Vol. v, 1852, 1']. 37, 3x. Issued in No. 2, April, 1851.

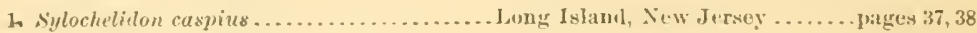

8.

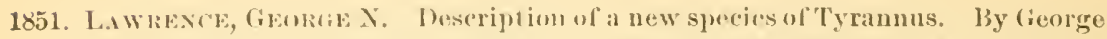

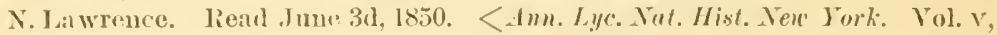
18.52, 1p. 39, 10, plite No. n of Tyramms cussinii, Lal wrence. Issued in No. 2, $\Lambda_{\text {pril }} 1851$.

1. Tyrannux cassinii, n. s............. Texas ................. pages 39,40

9.

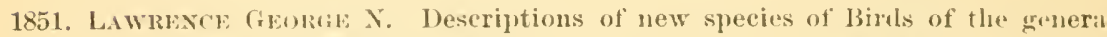
Conirostrum, Do(1)b, et Lafr., Embernagra, Less. and Canthornus, Jiriss., together with a List of ofleer species not heretofore noticeal as being fomm within the limits of the l'nited states. By George $x$. Lawrene. Reaul

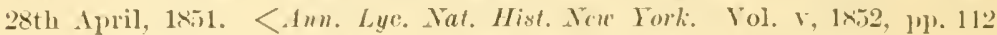
-117, plate No. v of Conirostrum ormatum and Embermagra ruficingata. Issued in No. 3, May, 1851.

TIREE NEW SPECIES.

1. Conirostrum ornatum, u. s.............. Rio Grande, Texas................... 11.

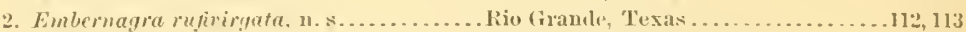

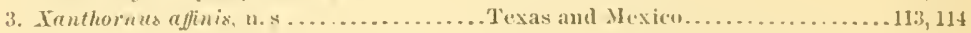

LIST OF BIRDA FRUM TEXAS (XEW TO THE V. S.) COLLECTED BO CAI'T. J. l'. M'COWN, U. S. A.

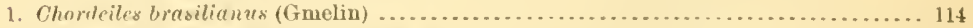

2. I'icolaptes brunneicapillus, La Frusnaje. . . . . . . . . . . . . . . . . . . . 114, 115

3. Pyrocephalus rubincus, Budk. - Inscicapa roronata, Gmel ................. 115

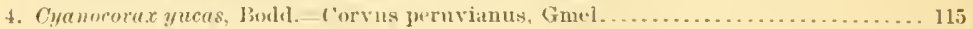

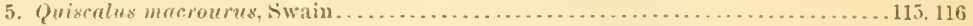

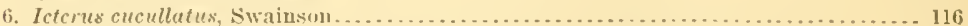

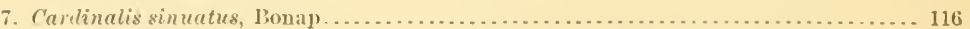

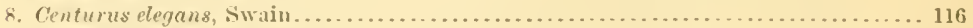

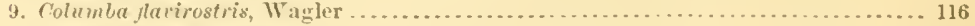

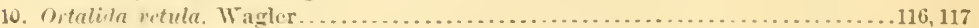

11. Dentrocygma autumalis (Liun.), Eyton ................................ 11.

10.

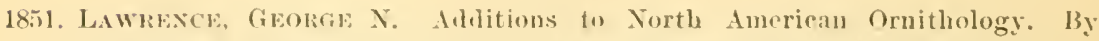
George N. Lawrener. Ni. 1. L.lnn. Lyc. Tat. Hist. Vew Jork. Vul. V. 1852, 1p. 117-119. Issterl in No. 3, May, 1881. 
11.

18.5. Lawrexce, GEorge N. Deseriptions of Now species of Birds of the Genera Toxostomal Wagler, Tyranmula Swainson, and Plectrophanes Meyer. By George N. Lawrence. Reacl sth sept., 185i. <Anu. Lye. Nat. Hist. New Sork. VoI. v, 1852, pp. 121-123. Issued in No. 4, September, 1851.

1. Toxo Prome.

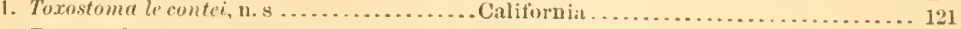

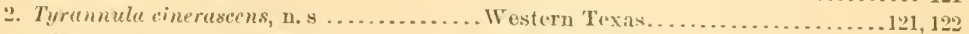

3. Plectrophaner me cownii, n. s..........W Western Texas.................. 122, 123

12.

1Ris. Lawriace, Geolite N. Additions to North Ameriean Ornithology. By fieorge

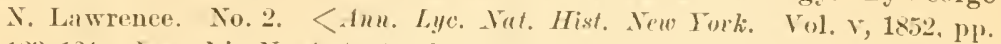
12:3, 124. Issued in No. 1. Feptember, 1851.

1. Centunus ont Page.

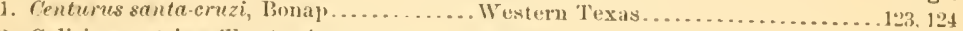

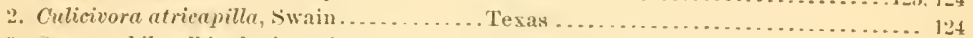

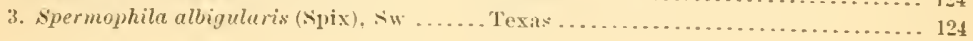

13.

1852. Lawrexce, Geurie N. Oruithological Nutes. Jy (ieorge N. Larrence. Reat April 24th, 18.2. <.tun. Lye. Nat. Hist. New York. Vol. v, 1852, pp. 220-223. Notes on nime species of North Ameriean birds.

1. Buteo harluni. and. Page,

2. Procellaria meridionalis, Lawrenew

1. Procela

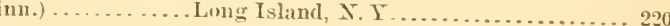

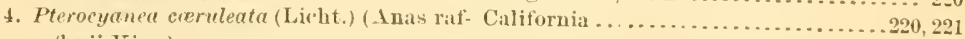
Hesii King).

5. Turdus nevius, Gmelin............... Vicinity of New Tork...........221, g9o

b. Ntrobilophaga enucleator (Lun.) ........... Massachusetts, Xew Jersey. Pennsyl. vauia, vicinity of New Fork Cily..... 222

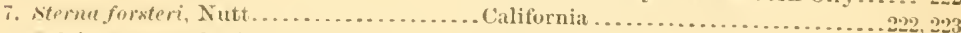

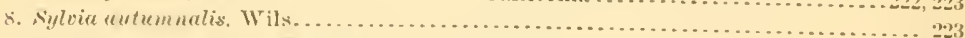

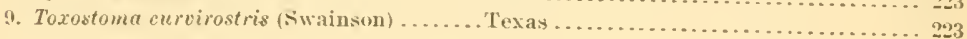

14.

1853. L.twexck, Faokge N. Deseriptions of New species of Birls of the fencra Ortyx Stephens, Sterna Linn., and leteria Vieillot. By George X. Lawrence. React Feb. 1.t, 1853. <Anи. Lyc. Nut. Hist. Net Tork. Vol. vi, 1858, pp. 1-t.

Page.

3. Icteria longicauda. n. s.

15.

18.3. Lawrexce, George N. Adlitions to North American Ornithology-No. 3. By George N. Lawrence. Real March 2x. 1853. <Anu. Lyc. Tat. Hist. Nine Tork. Vol. 11, 18: 5, pp. 4-7.

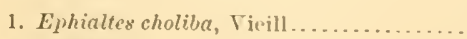

2. Puffinux ?

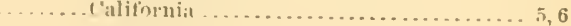

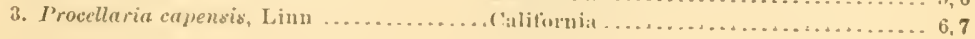


16.

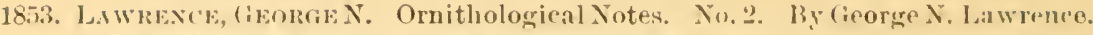

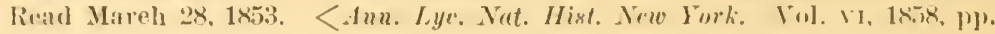
$7-14$.

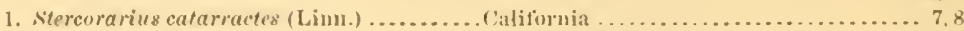

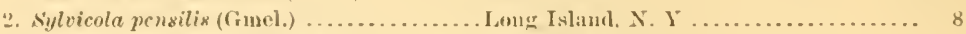

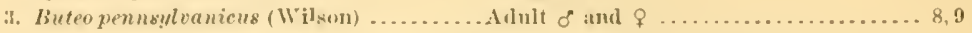

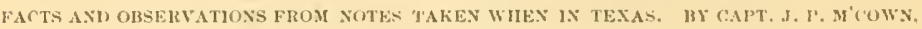
U. \&. ARMY.

1. Conirostrum ornatum, Lawrenee.................................... 9

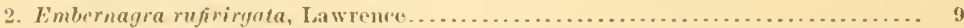

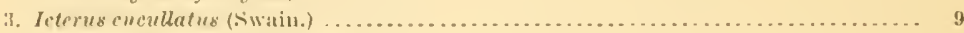

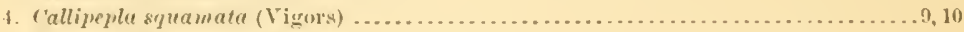

5. Cyrtonyx masena (Less.) ............................................ 10

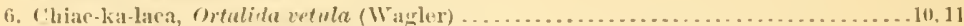

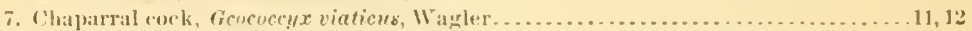

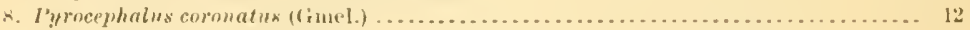

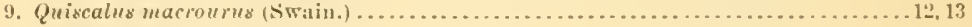

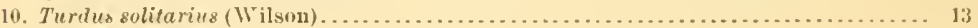

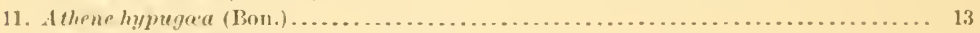

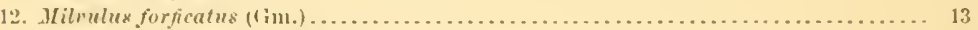

13. ("yanocorax luxuosus (Lesson) ...................................... 13

14. Columba flavirostris, Wigler........................................ 13

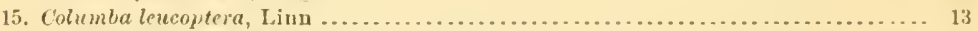

16. Macrorhamphus scolopaceus (Say), lawrenpe ........................... 14

17. Plectrophancs me cownii, Lawrence ................................. 14

18. Tyrannula cinerascens, Lawrence ..................................... 14

17.

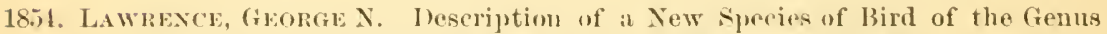

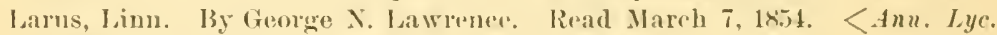
Nat. Hist. Now Fork. Vol. vi, 185s. plp. 79, 80.

1. Larus califormicus, n.s. pages 79,80

\section{8.}

185\%. Lawrince, Genkge $N$. Deseription of a New Species of Humming Bird of thu fienus Mellismga Brisson, with a note on Trochilus aquila Bonreier. By George N. Lawrence. Real April 2, 1855. <Anu. Lye. Nat. Hist. New Jork. Vol. VI, 1858, pr. 137-142, plate No. IV of Mellixuya ulbo-coronala. Issued in No. 5, Octoher, 15.5.

1. Mellisuga alho-cornuata, n. s............. Veragnas, New Granada

Page

2. Trochilus aquila, Bourcier. Verilguas, New Granalia 137-139 $139-142$

\section{9}

1856. LAwrisce, (reorge N. Descriptions of Sew Speeies of Birls of the Genera (hordeiles, swainson, and Polioptilit. Selater. By Genrge X. Lawrence. Rearl $22 d$ Derember, 18int. <Ann. L.yc. Nut. Hist. New Fork. Vol. VI, 1858, ㄱ. $16.5-169$.

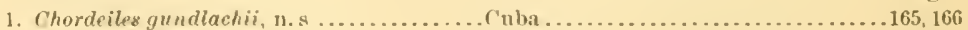

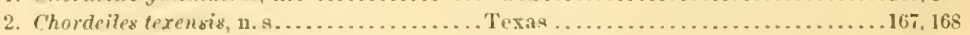

3. Tolioptila melanura, n. s.............. Texas, Califoruia ..............16 . 169 
20.

1858. LaWRFXCE, Glokge N. Deseriptions of Seven New Species of Humming-Birds. By George N. Lawrence. Read Fehruary 15th, 18in. <.lun. Lyc. Nat. Hist. Vere Jork. Vol. vi, 1858, p1). 258-264.

Page,

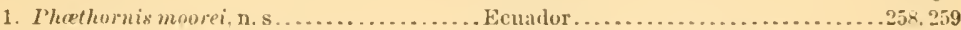

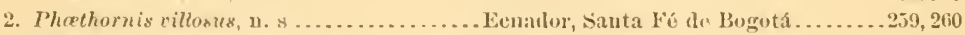

3. Phethomis atrimentalis, $11.4 \ldots \ldots \ldots \ldots$. Ecuator....................... 260

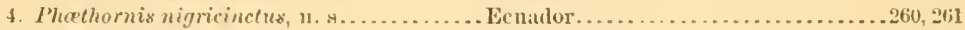

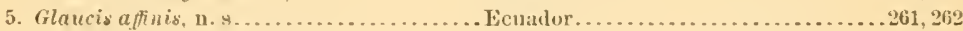

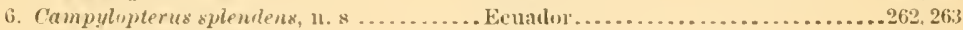

7. Ionoluima frontalis, n. s.............. Eenador......................263, 264

21.

185x. Lawrexce, Geolias X. Deseriptions of two New Spreies of Gutls in the Insem of the Smithoonian Institution at Washington. By George N. Lawrence. Real June 29th, 1857. <Ann. Lyc. Nitt. Hist. New Fork. Vol. VI, 1858, pl. $261-266$.

Page.

1. Larus suckleyi, n. s.................... Puget Siund, Nortl Pacific........264, 265

2. Riswa septentrionalis. $11.8 . . . . . . . . . . .$. . Pacitic ('oast of North America, Puget

sound............................ 266

22.

1858. Lawrexce, GEokg: $\mathrm{N}$. Observations on the preceding Paper (one hy Johu Gmudlach, Ph. D. "Notes un some Culsan Birds, with Descriptions of three New species"). <Ann. Lye. Sat. Hist. Neu Tork. Vol. vi, 1858, pl). 275-277. OBSERVATIONS ON FOUR MTIN IHIRS MEXTIONEN IN DR, GUNOLACI'S PAPER.

Page

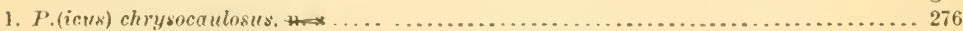

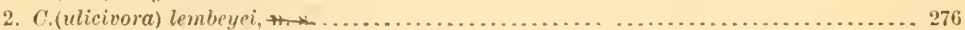

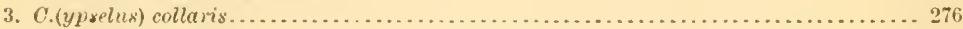

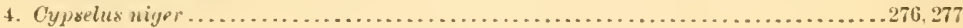

23.

1858. Lawrexce. Grorgr: N. Longipennes, Totipalmi, Brachypteri. By George N. Lawrence. CExplurations and surveys for a railioal route from the Mississippi River to the l'arific Geean. Vol. Ix, War Department, Birds, Washington, 1858, 1"p. $8: 20-8 ! ! 9$. Reissued."

\section{LONGIIENNES.}

PIUCELLARIU.E.

Page.

1. Diomedu exulanx, Limman............. Pavific Ocean.................... 821

2. Iriomeda brachyura, 'Temminck .......... Nurth Pacidie teean, coasts of California

and $($ regron........................ $8: 2$

* Batrd, ‥ F. The Birls | of | North Ameriua; the deseriptions of species based chielly on the col-

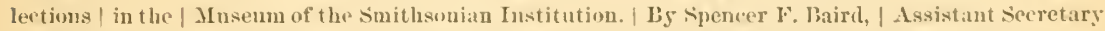

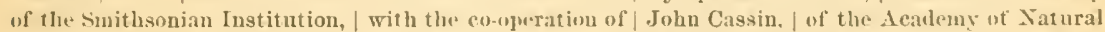
Sciences of P'hilatelphia, | and | George $N$. Lawrenee, fof the Lien of Natural History of New York. With an atlas of one huntred plates. | Text. | - | Philadelphia: | J. I3. Lippincott \& Co. | 1860. 4to. - p. ill. (Title, Adrt.), pp. i-lvi, 1-1005. Atlas, pll. 100. A reissuc, retitled, of Vol. Ix, Pacific Railrond Reports, $1858, q . v$. The main text is ilentical, apparently from tho same plates; and so the

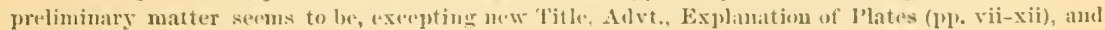

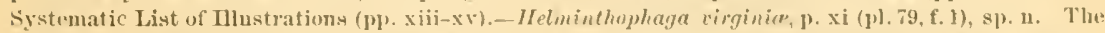
Atlas d'onsigts of 100 pll., alwoll half of which an" new, the remainder heing from the l'acific Railroad Reports and Mexican Boundary surves, returelwel aud relettered, in amme catses reelrawn, for this edition. Bibliographical Appendix to birds of the Colorado Valley. I'art first. Ly Elliot Couses, p. 65ö, Washingtou. 1878. 


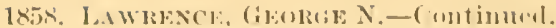

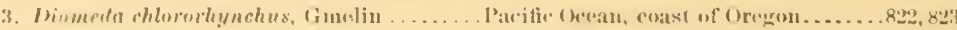

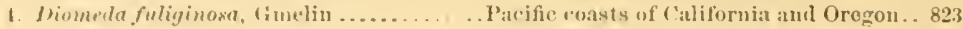

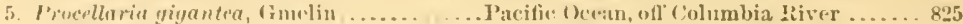

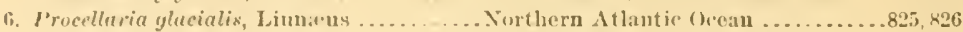

7. Procelluria pucifiea, Audulon ...... . . l'acifoc coast of Nurth Auerica........ 826

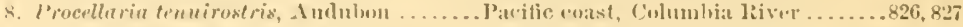

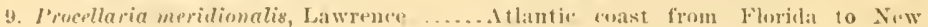

York ........................ 827,828

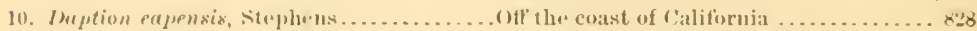

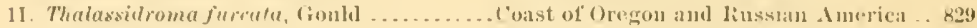

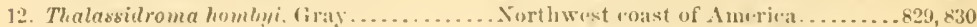

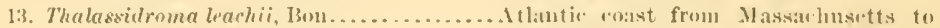

Battius bily..................... $8: 30$

14. Thalassidroma melnuia, Bonapartı ....... Coast of California .............. $\times 30$

15. Thalassidroma wilsoni, Bontunte ........ () the Atlantie cuast from the Gulf of

Ifexien to batlins Bity .............. 831

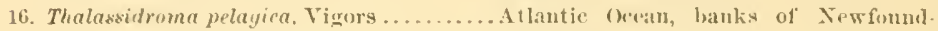
b:ud ............................. $831,832_{2}$

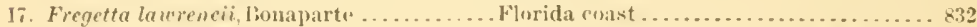

18. P'uffinus major (Faler) ................ Atlautic Ocean, Florida coast to the Gulf of st. Lawrences ................... 833

19. I"ufinus fuliginosus, Strickland ........... Allantic coast of the Forthern States,

banks of Jewtoumland.............. 834

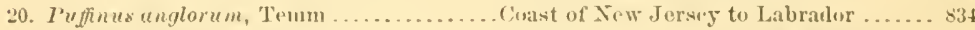

21. I'uffinus obscurus, lathinn...............

of Jixico .......................... $\times 33^{5}$

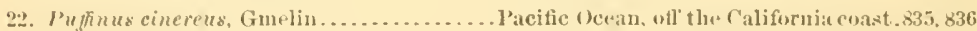

\section{LARIDE.}

23. Stereoraries catarractes, 'Temm ......... ('bast of California ............... 838

24. Ntereorarius pomarinus, 'Temminck...... Lalmador, as far south as Now York in winter, 11 arrishurg, $\mathrm{P}: 1 \ldots \ldots . . . . . \times 38,839$

25. Ntercorarius parasiticus, Temminek........Arctic America; const of the Enited States from New Jork northwari .... 839

26. Stercorurius cepphus (Briinnich)..........Aretie seacoast.s of Anerien; Bathins liag ............................. 840

27. Larus glancus, Brïnuich ................ Aretit seas, Labrador; New York iı winter rare $]$.................... 842

2.. Larus glauceseens. Tichtenstein.......... Nortluwest woast of Forth America... 842, 843

29. Larus lcuenpterus, Fabr. ............. Ireic seas. Baltins Bas, Labrador..... 843

30. Larus ehalcopterus, Licht................ American coast of Bering Strait and (irtenland ....................... 843

31. Larus marinus, Linnatus............... Nortl Atlantic, Labralor; as far sonth as Florila in winter.............. 84

32. Larus urgentatus, Brümuich

Allantic cuast from Texas to Newfound land; Western states; Ohio and Mississippi rivers...................844.845

33. Larus oceidentulix, Aulubon ............ Nurthwest coast of Nort h America..... 845

34. Larus californicus, Lawrence..........W West coast of Jorth America........ \& 46

35. Lams delauarensis. Ort ................. Aretic Anerica; 'Texas to Labrador; western rivers; nort hwest coast. ...,466,847

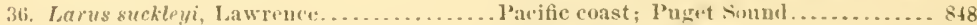

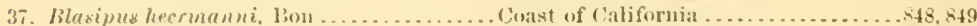

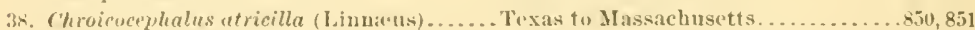

39. Chroiencephalus franklinii, Bruch .......... Missonri River, interior of fur entries. 851

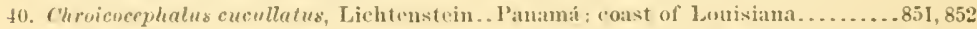

41. Chroicocephalus bhiladelphia (Ord.)...... Texas to Novascolia; Mississippi River: fur eomntries; l'acifie roast of North

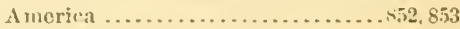

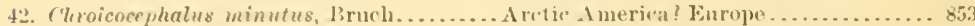

43. Rizza tritactylus, Jonap ................. Fur count ries; Lahmadur : sonthern eoast in wiıler..........................

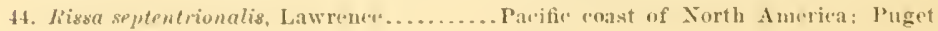
sonnd 


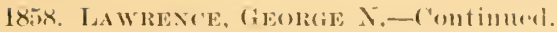

Page.

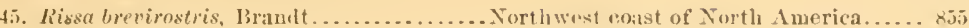

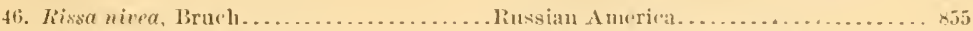

47. Pagophila eburnea, Kaup................. ('onsts of Aretic America, Labralor,

Newfoundlund .................... 856

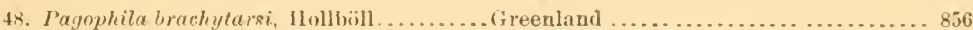

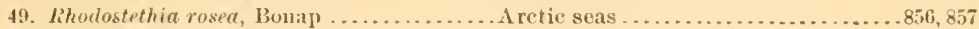

50. Creagrus fureatus, Neboux ........... California .................. 857

51. Fema sabinii, Sabino ................... . Nova Seotia nortl warel; Aretir seas ..... 857

52. Sterna aranea. Wilson................. Cuast of the Lnited States as fir north

as Comerticut .................... 859

53. Sternu easpia, Pallas .................. Coast of New Jersey northward....... 859

54. Sterna regia, Gambel ................... Atlantie "oast of the Southeru and Mid-

Ill: States aud California ......... . 859,860

55.) Nterna elegans, Gambel .................. Pacific coust of Mi'xico; coast of southern

Cillifornia, nort hern Califoruia? ...... 860

56. Sterne aeuflavida, Cabot ................ Texas to Florilla . . ............... 860

57. Sterna havellii, Andubon ............... Texas to South (Caroliua . . .......... 861

58. Sterna trudeanii, Aulubor .............. Coasts of Now Jersey anil Long Island. . 861

59. Sterna fuliginosa, Gmelin.............. Texas to Florida ................. 86I

60. Stema wilsoni, Bonaparte .............. Texas to Labrador ................ 861, 862

61. Sterna macrura, Naumanm ............. Coasts ot the New England states to

Aretic seas; fur countries.......... 862

62. Sterna forsteri, Nnttall

Louisiana to Florida: New Tork, fur countries, and California..........862,863

63. Sterna paradisea, Briiunieh............ Florida to New York............. 863

64. Sterna pikei, Lawrence ................ Coast of California . . . .............. 863

65. Sterna frenata, Gambel................. Texas to Labrador; Westeru rivers...... 864

66. Hydrochelidon plumbea (Wilson) ......... Texas to the New Hugland States; Mississipyi Riverand tributaries; furconntries. .......................... 864,865

fii. Anous stolidus, Leach................ . Texas to Florida . . . . . . . . . . .... 865

68. Rhynchoys nigra, Linurens ............ From Texas to New Jersey .......... 866

\section{TO'TIPALMI.}

PELECANIDE.

69. Pelecanus erythrorhynchus, Gmelin ....... Tnited States; raro on cuasts of Mrildle and Northeru States; fur countries up to the 61 st parallel ............... 868,869

70. Pelecanus fuscus. Linnasus.

Frum Texas to North Carolina; Californiat coast ..................... 870

STLIDE.

71. Sulu bassana (Linnarus) ................. Atlantic coast from Lalorador to the Gulf of Mexiro .................. 871,872

72. Nula fiber (Linneus)

Gulf of Mexico: - Hlantic coast from

Georgia southward................ $87^{2}$

TAC'ISPETID.E.

73. Tachypetes aquilus, Vieillot ........... Texas to Florita ; Calitornia .......873,874

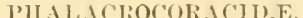

74. Graculus carbo, Gray .................. Labrator and Atlantir coast as fiur south as Jew Jersey in winter ........... 8,6

75. Graculus perspieillatus, Pallas......... linswian Ameriq $\ldots \ldots \ldots \ldots \ldots \ldots \ldots .877$

76. Graculus cinciunatus. Gray ............. Ninthwest eoast, Sitha.............. 87 T

77. Graculus dilophes, Gray ................. Atlintio coast from Labrador to Caroliun: fiur countries: Pacitic coast from Washingtou 'Torritoryto ('alitornial 877.878

TR. Graculus foridanus, Bon Aloue the coast from Texar to Florida; Mismissifpi liver............... 879

79. Graculus mexicanus, Bon .. Texils on the lio cirande; Cuba......879, 880 


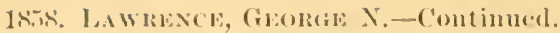

s0. Graiulus penticillutus, Gray

West coist of Xorlh $\Lambda$ muriea $\ldots . . . .8660,881$

81. Oraculus violacens, liray.

Weslerin coast of North Ameriea; Cali-

fornia; W:ashington 'Jerritory...... $\times$ nl, 832

JUOTIDA

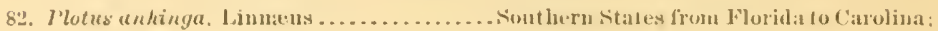

"Texas ......................... $88.3,894$

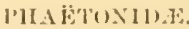

83. Thäton flavirostris, lsandt...

Floridlit roast . . $.8 \times 5,886$

\section{BRACHYTIERI.}

CULYMBIIN.

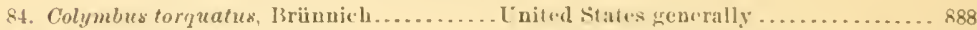

85. Colymbes arcticus, Linmaus .............. Texas; Columbia River ? ........... . 888, 889

86. Colymbus pacificus, n: s................. Coist of Califuruia ; Puget Somud .... 884,890

87. Colymbus septentrionalis, hinn ........... Duriner the winter ats fir soul lo as Marylitud; Arctie seas; l'acifie crast. . .890, 891

sx. I'oticeps griseigena Gray. Fur countries; in the Atlantic siates as litr south as Pcunsylvania, in win-

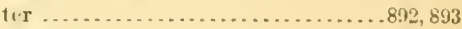

89. I'viliceps cristatus, Lath Atlantic: States from Novia Scotia southward; Texas in winter; fur conntries; Pacitit side of the contin'nt; Washing ton T'errit ory .................. 893.894

91. Poticeps occidentalis, n. s.... ........... Pacific coast from Washington Territury to Ciliforniat ..................... 894

91. Puliceps clarkii, n.

California anu New Mexico ........... 895

9:. Podiceps cornutus, Lath ............... United States germ rally . . . . . . . . . 895,896

93. I'odiceps californicus, Heermann...........California; heas] waters of Missouri liver . . . . . . . . . . . . . . . . . .896, .997

94. Iodieps auritus, Latham ............... Aretic America ? .................. 897

95. Jodilymbus porliceps, Lawrenco........... Aflantic States geuerally; Texas and New Hexico; California and ()regon.....898, 899

24.

1860. Lawmexce, Glomge $\mathrm{X}$. Deseriptions of three new species of Jlumming-loirls of the finera lleliomaster, Amazilia, and Mellisuga. By George N. Lawrunce. Rual Ipril !, 1860. <Anu. Lyc. Wat. Hist. Wew Fork. Vol. vil, 1862, pp. 107-110. Issued in Nos. 4-9, April-May. 1860.

Page.

1. Meliomaster stuartee, n. \& ............... Santa Fé de Bugoth. Now Grunada...107-109

2. Amazilia xantusii, 11. s ................. Cape St. Lucas, Soutl C:lliformia....109, 110

3. Mellisuga merrittii, n. \& .............. Veraguas, New Grauda..........110,111

\section{5.}

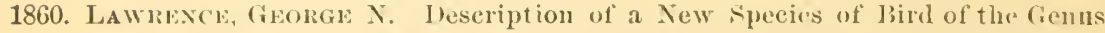
Phatou; also of a new spucies of Ilumming Birsl of the grems Jleliopirdicat. By George $\mathrm{N}$. Lawrence. Read April 23, 1860. <.1nn. Lye. Iut. Hist. Wew Fork. Vol. vil, 1862, 1P. 112-145. Issued in Vo. 4-!), April-dlay, 1860.

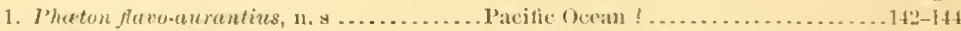

2. Ileliopedica cartareocauda, n. .......... Cape St. Lueas, Sout li California...... 145

26.

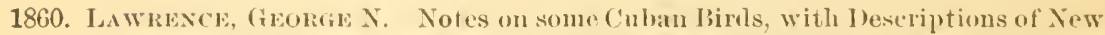
Spereirs. liy fororge X. Isawrence. liead May 21, 1860. L.Inn. Lye. Vut.

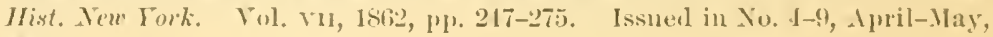
1860 .

1. Tiиnunculus sparveroiles (Vigors) .....................................

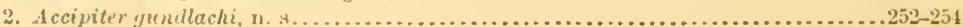




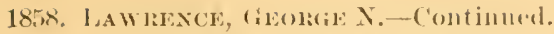

3. Accipiter fringilludes, vigurs . . . . . . . . . . . . . . . . . . . . . . . . 255-25i

4. Cymindis vilsoni, Cassin ....................................... 257

5. Gymnoylaux nudipes (1)audin) . . . . . . . . . . . . . . . . . . . . . . 25i-259

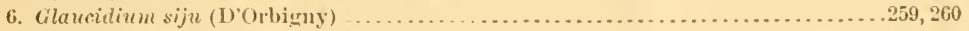

7. Antrostomus exbanensis, $\mathbf{n}$. . . . . . . . . . . . . . . . . . . . . . . . . . . . . 260-262

8. Churdeiles minor, Calmuis......................................262, 2633

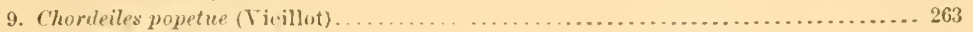

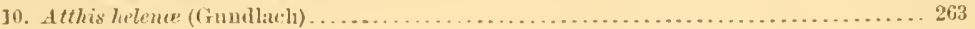

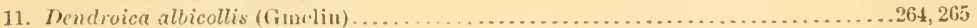

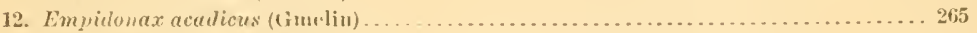

13. Virensylvia altiloqua (Vieillot) . . . . . . . . . . . . . . . . . . . . . . . . . . 266

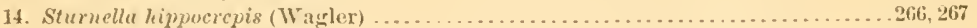

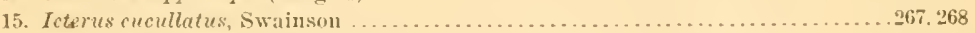

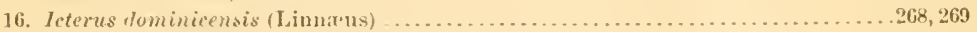

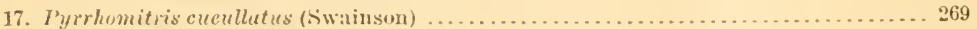

18. Spizella socialis (Wilson) . . . . . . . . . . . . . . . . . . . . . . . . 269, 270

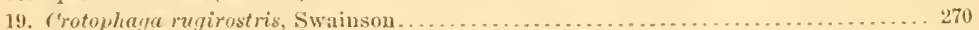

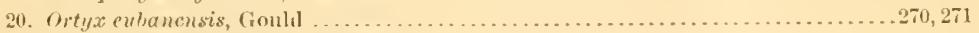

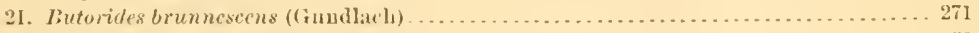

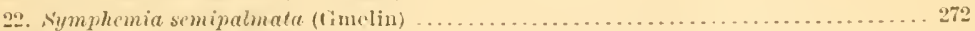

23. Haceramplius seolopeceus (Siny) . . . . . . . . . . . . . . . . . . . . . . . 272, 273

24. Nacroramphus griseus (Gmeliu) . . . . . . . . . . . . . . . . . . . . . . 273

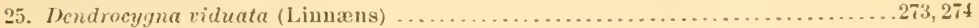

26. spatula clypeata (Linurus) . . . . . . . . . . . . . . . . . . . . . . . . . 274,275

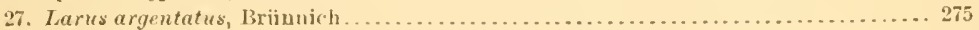

27.

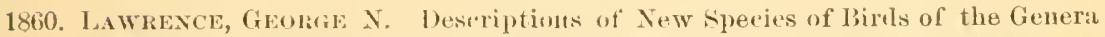
Myiarchns and I'hlogopsis. By (ien)rge $\mathrm{N}$. Lawrence. Read 21st May, 1860. <Auu. Lyc. Nat. Mist. New Yorl. Vol. Vil, 1862, 12. 28.1-286. Issned in No. 4-9, April-May, 1860.

Page

1. Myiarchus punamensis, n. s ............ Isthmus of Pamamá ....... . . . 284,285

2. Phlogyopsis me lecennani, и. . .......... lsthmus of P’anamá ............285, 286

28.

1861. LAwrexce, Grolat: N. Catalogute of a collection of Birds, made in New Grenada. By James Mcleamman, Esq., of New Jork. With Notes and De-

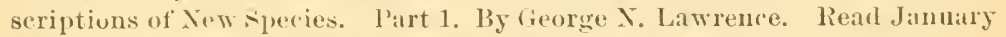
28th, 186I. Ltun. Lyc. Tat. Hist. Sew Fork. Tol. V11, 1862, pl. 288-302.

FALCONIIT.K.

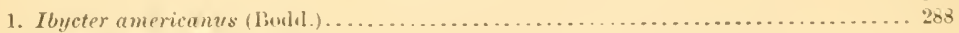

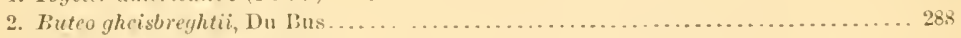

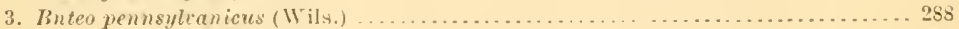

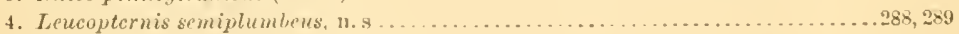

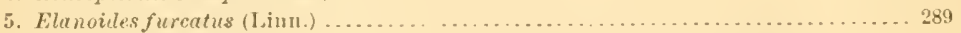

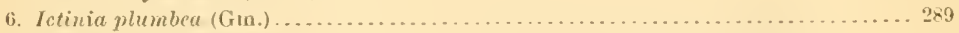

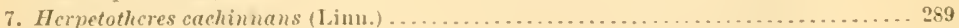

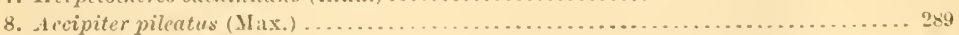

9. Geranospiza derulescens (Vieill.) .................................. 289

STIITIII).

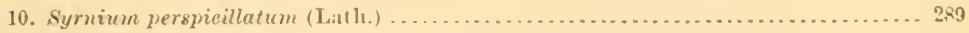

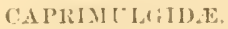

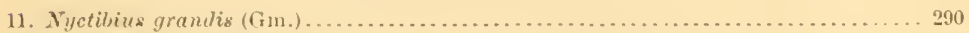

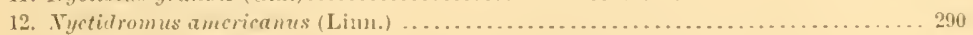

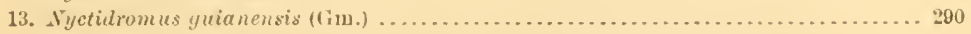




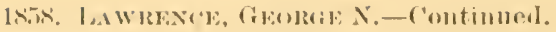

MUMUTIOA:

Page.

14. Momotus martii (Svix.) ...................................... 290

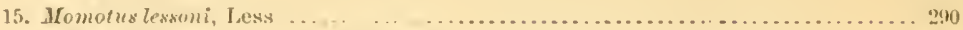

16. Crypticus platyhumehus $\{$ l.

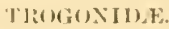

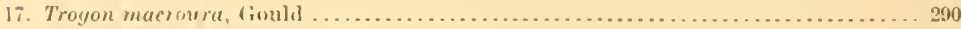

18. Trogon massena, Gonlı1......................................... 290

19. Trogon virilis, Liun.............................................. 290

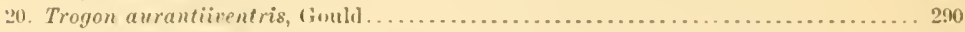

21. Trogon ealigatus, (ioulı ....................................... 290

HIC(O)XID.

22. Malacontilu panamensis, Lafr.

Al('WIINIII:

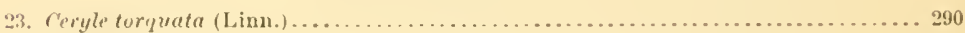

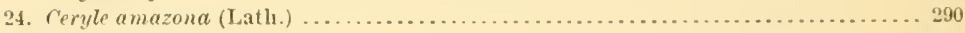

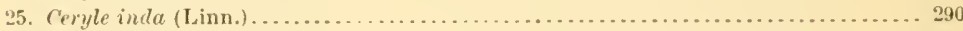

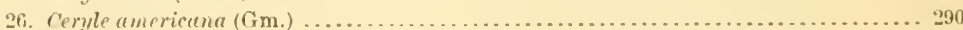

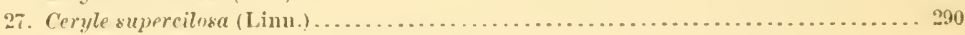

(AILBIIII) $\mathrm{F}$.

28. Jacameraps grandis (Gin.)

\section{C.FIEBII)}

29. Coereba carneipes, Sel. .......................................... 291

30. Careba lucida, Sclater and Salvin . . . . . . . . . . . . . . . . . . . . . . . . . . . 991

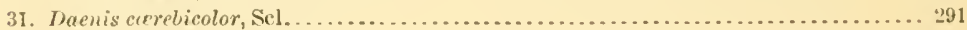

32. Certhinla luteola, Całan ......................................... 291

'PROC'HILII.E.

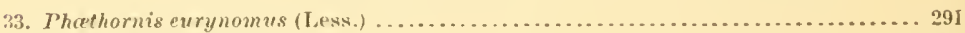

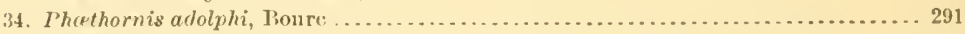

35. Heliothrix auritus $(\mathrm{Gm}$.$) . . . . . . . . . . . . . . . . . . . . . . . . . . . . 291$

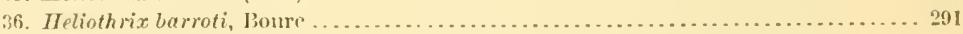

37. Heliomaster stuarte, Lawr . . . . . . . . . . . . . . . . . . . . . . . . . . . . 291

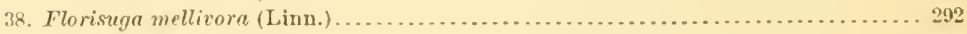

39. Thalurania venusta, foult . . . . . .

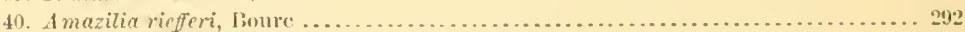

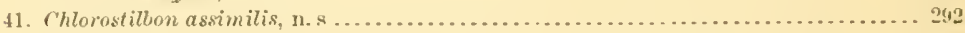

42. Erythronota edvarti (lourr. et Delatt.) ............................ 292

43. Juliamya typica, Bonap.............................................

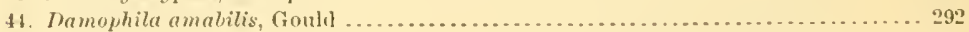

\section{C'ERTIIIDE.}

45 Rhodinocichla rosea, I.ess. . . . . . . . . . . . . . . . . . . . . . . . . . . . 292

46. Dendrornis guttatus (Licht.) . ................................... 292

4. Dendrornis tennirostris (Licht.) . . . . . . . . . . . . . . . . . . . . . . . 292

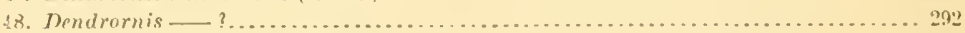

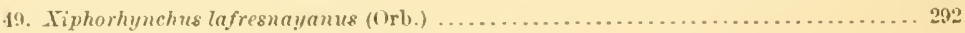

TIROGLODITI1).

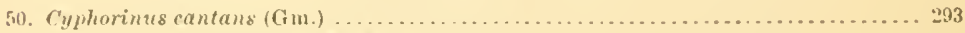

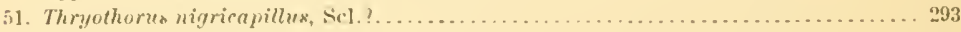

MNHTILID,

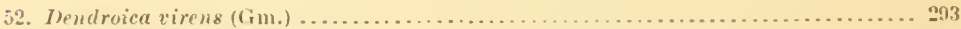

53. Helminthophayg shrysoptera (Linn.) ................................. 293 
1858. LAwrexce, Georit N.-Contimuel.

FOLMICARIINE.

54. Cymbilanius lineatus (Leach) ..... Page.

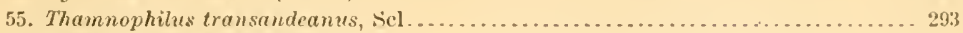

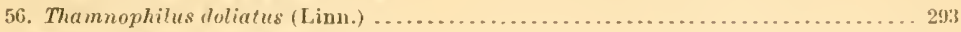

57. Myrmetherula surinamensis (fin.) . . . . . . . . . . . . . . . . . . . . . 293

58. Pithys rufigularis (Bodd.) ?..................................... 293, 294

59. Gymnocichla nudiceps, Cassin. . . . . . . . . . . . . . . . . . . . . . . . . . 294

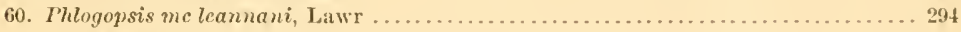

61. Pitusoma michleri, Cass ....................................... 294

ANABATII.E.

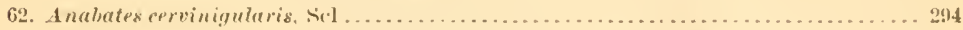

TYRANNIDA.

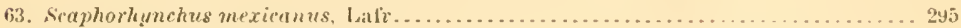

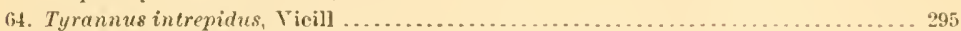

65. Tyrannus melancholicus, Vicill. . . . . . . . . . . . . . . . . . . . . . . . . . 295

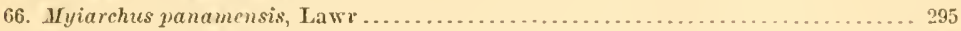

67. Myiodynastes nobilis, Sel ............................................

68. Milvulus monachus, Hart] ......................................... 995

69. Myiozetetes cayenneusis (Linn.) . . . . . . . . . . . . . . . . . . . . . . . . 295

70. Todirostrum cinereum (Linn.) . . . . . . . . . . . . . . . . . . . . . . . . . . 295

71. Muscivora mexieana, Scl ...................................... 295

COTINGID.F.

72. Tityra albitorques, 1) Bus . . . . . . . . . . . . . . . . . . . . . . . . . . 295

73. Tityra personata, Jard. and Sclby . . . . . . . . . . . . . . . . . . . . . . . . 295

74. Pachyrhamphus cinnamomeus, n. s............................... 295, 2906

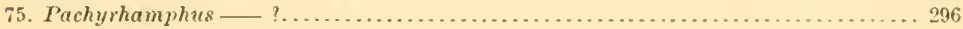

\section{IMPELIDE.}

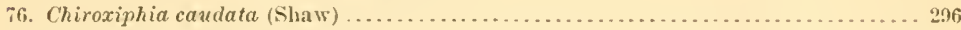

77. Miroxiphia mentalis, Scl....................................... 296

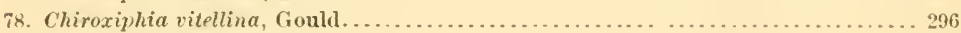

79. Chiroxiphia cyaneorapilla, Wagl ................................ 996

80. Querula eruenta (Botld.).......................................... 296

('IRTII).F

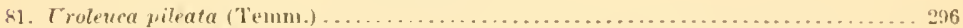

STTRNII).E.

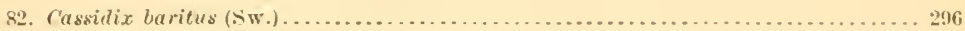

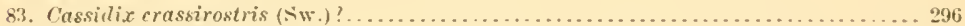

81. Ostinops montezuma, Lisg ....................................... 297

85. Ostinops cristatus $(\mathrm{Gm}$.) . . . . . . . . . . . . . . . . . . . . . . . . . . . . . 297

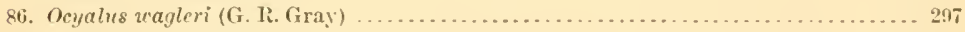

87. Cassiculus prevosti (Less.) . . . . . . . . . . . . . . . . . . . . . . . . . . . . 29i

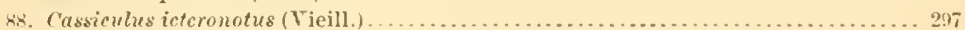

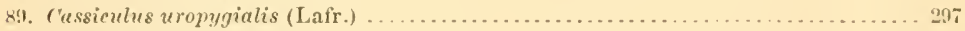

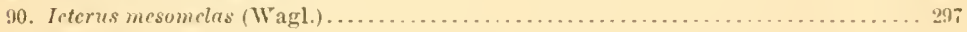

91. Icterus yiraudii, Cassin . . . . . . . . . . . . . . . . . . . . . . . . .

FIRINGILI,II).1:

92. Cyanoloxia eyanoides (Lafr.) . . . . . . . . . . . . . . . . . . . . . . .

93. Hedymeles ludoricianus (Wils.) . . . . . . . . . . . . . . . . . . . . . . 297

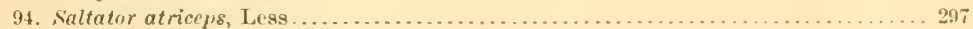

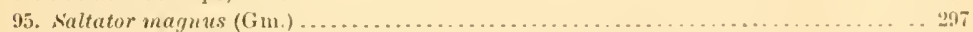

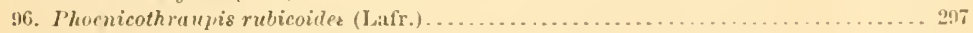

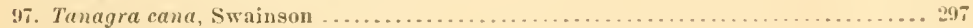

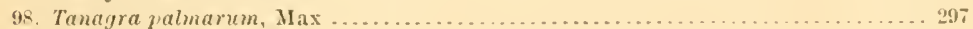

99. Rhamphocelus icteronotus, Bonap................................. 297

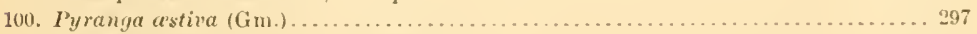

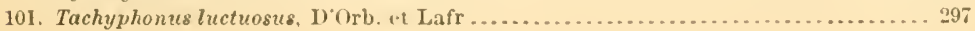




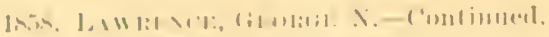

l'agi.

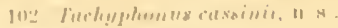

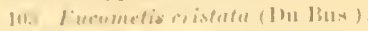

lu1 C'ullixte inurnatu, lionld

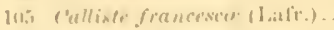

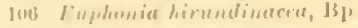

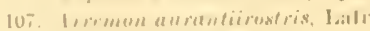

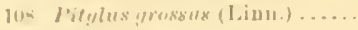

14!! Enspizu americana (litu.)

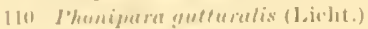

111. I'homipare pustllu (Sw.) ......

$.297,298$

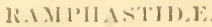

11:. Rihamphastus twertad, Virill.

299

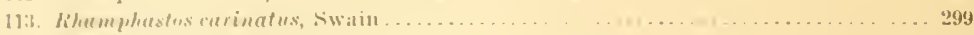

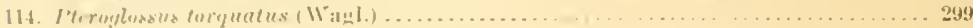

\section{I'SI'IAIII, X.}

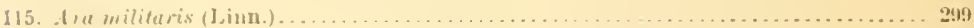

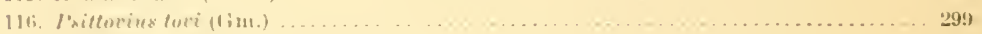

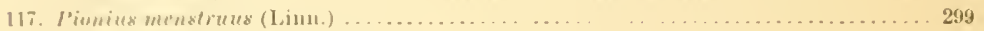

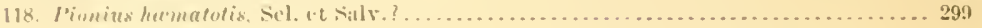

\section{I'ICII)A.}

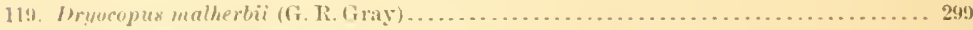

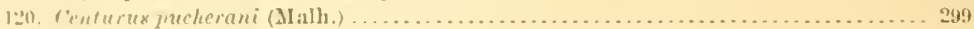

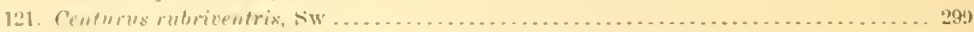

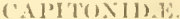

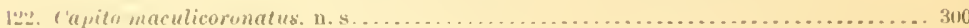

\section{C'CCTLIIIE.}

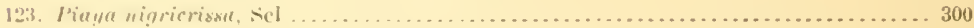

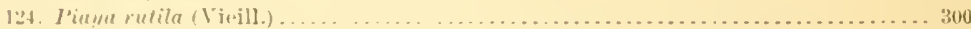

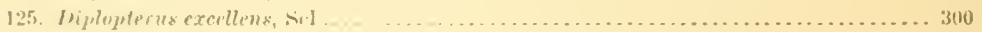

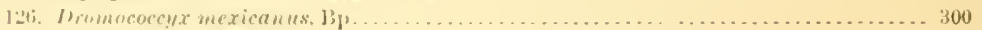

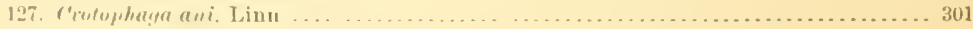

(1)L MBII).

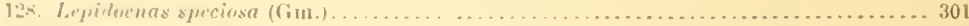

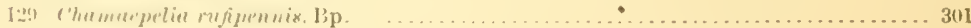

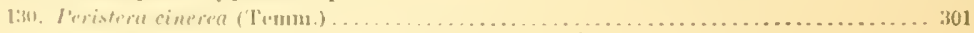

('I:A ('I1).]:

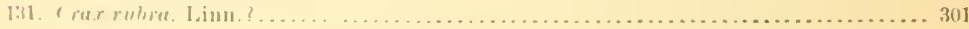

THTR.MNTH.E.

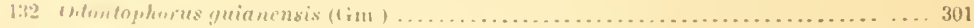

AliDIIJ).T:

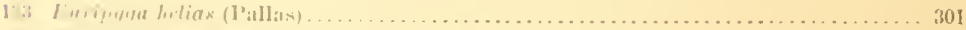

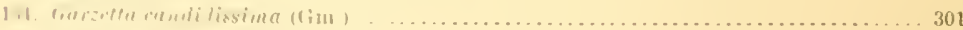

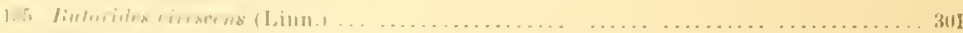

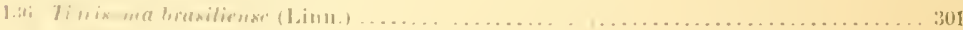

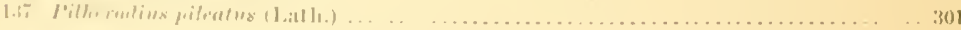

S1 $111,119.1111) .1$

I in rellina!n milanuii, Inp. 
1858. LAWREXCE, (iEuRGR N.-Contiumed.

\section{R.ILLIIIE.}

Page.

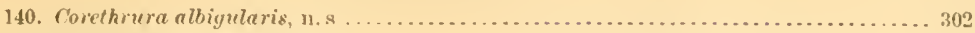

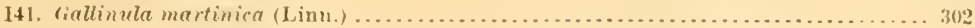

\section{(O) YMBIIDS}

142. Heliornix fulio (liosla.)

29.

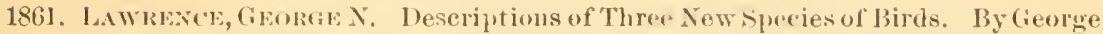

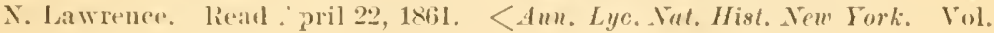
เ11, 1862, 1p. 303-305.

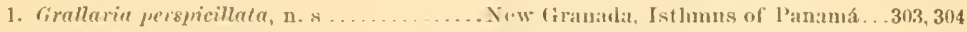

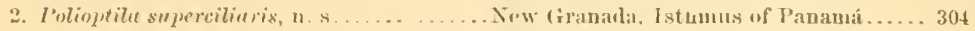

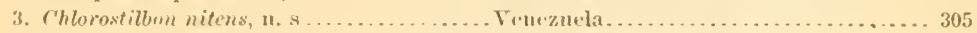

30.

1861. Lawresce, Geones $\mathrm{X}$. Catalogue of a Colleretion of birels made in New Grenada, by James MeLeannan. esq.. of New Vork, with Notes and Descriptious of New species. l'art 11. By George $\mathrm{N}$. Lawrence. Read May 27, 1861. <dun. L.ye. Nat, Hist. Nen Fork. Vol. Vu, 1862, pp. 315-334.

\section{FALCONIILE.}

Page.

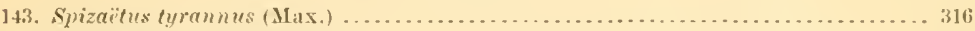

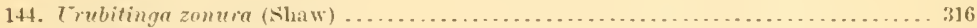

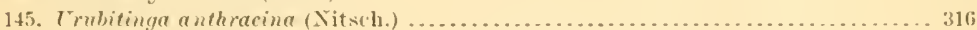

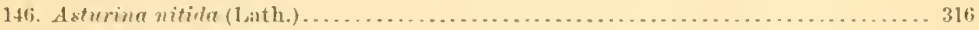

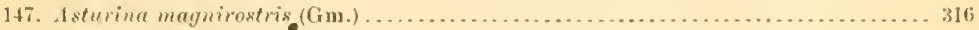

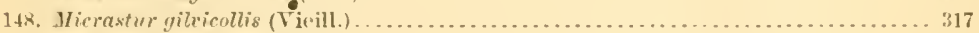

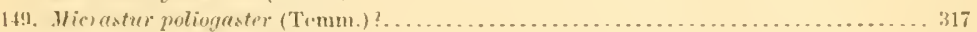

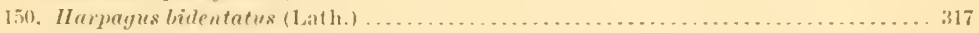

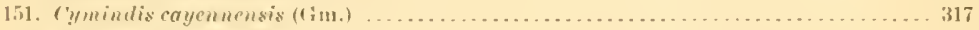

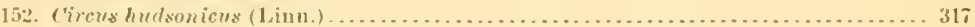

\section{S゙FIRIUE.}

153. simnium virgatum, Ciss . .

IIIINIXIDN.

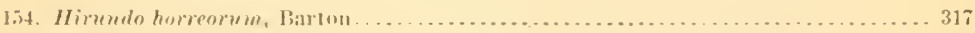

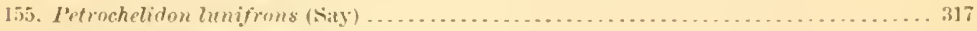

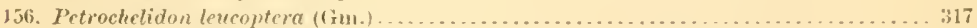

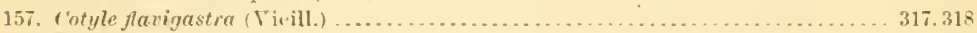

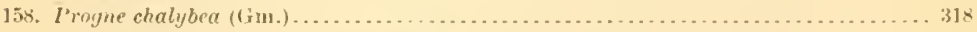

\section{MIUMTIDE.}

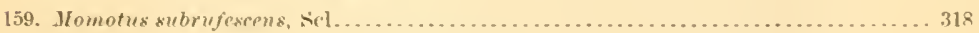

BCCCONID,E.

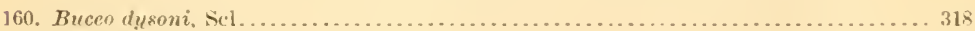

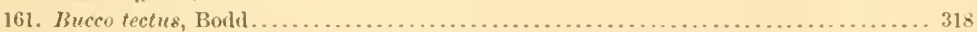

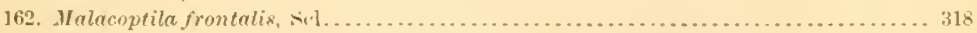

ALCENINID.E.

163. Feryle alcyon (Linn.)

CEREIIID.E.

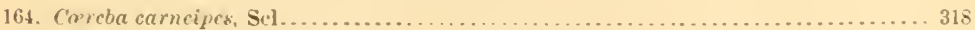

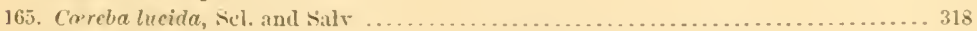

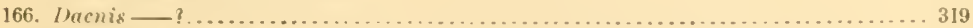

167. Chlorophanes atricapilla (Vieili.) ..................................... 319 


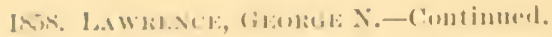

TTUCIILIDE.

Page.

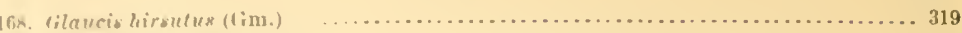

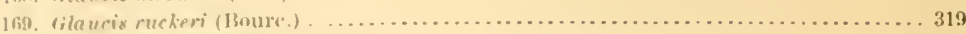

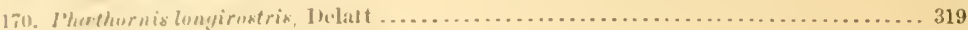

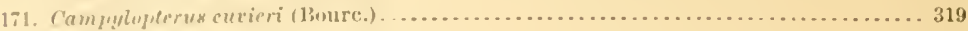

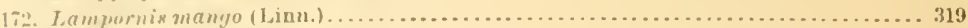

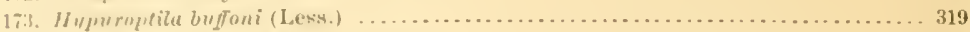

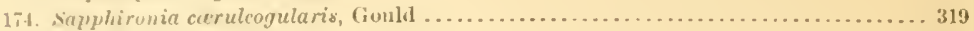

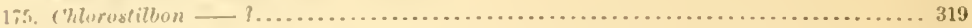

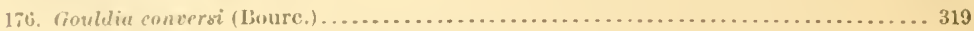

\section{ANABATIDE.}

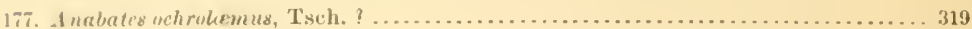

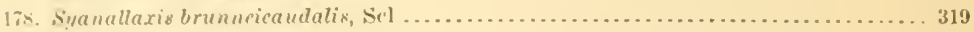

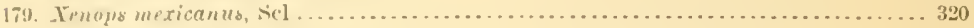

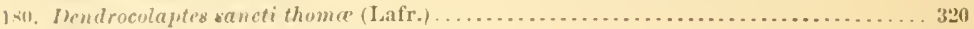

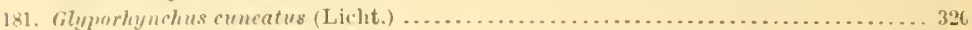

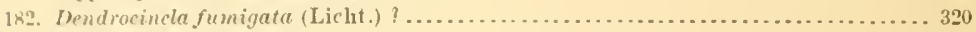

183. Dendrocincle — ?.................................................. 320

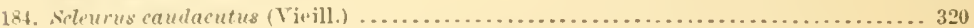

TROGLOINTII.F.

1.5. Cyphminus bambla (Bodl1.) ? ................................... 320

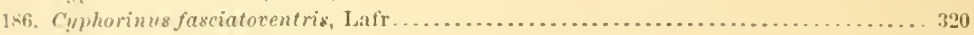

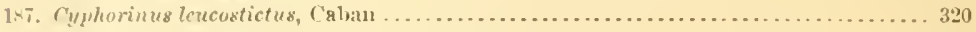

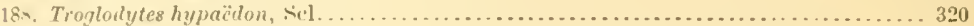

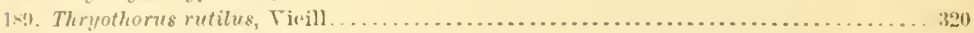

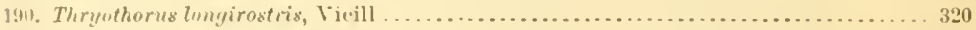

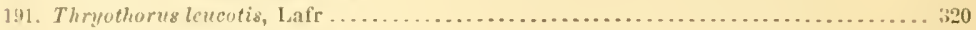

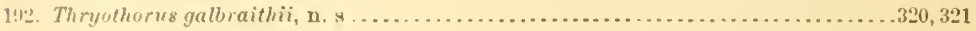

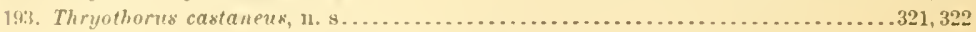

\section{MOTARILLIDE.}

194. Inthus rufus (Gm.) 322

PARID.F.

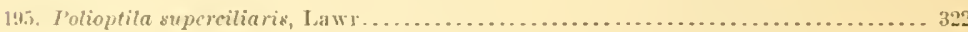

\section{MNIOTILTILE.}

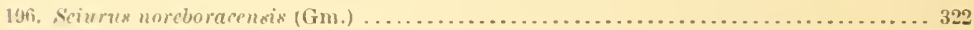

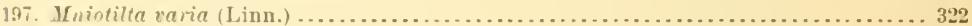

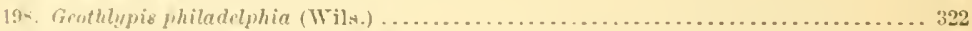

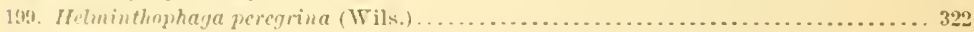

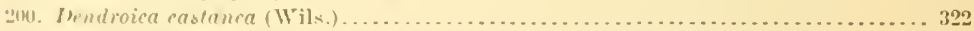

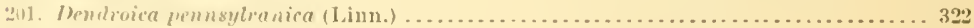

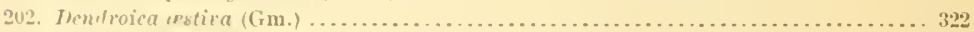

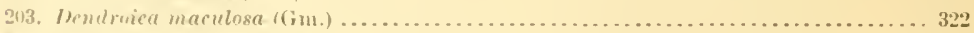

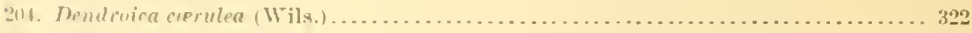

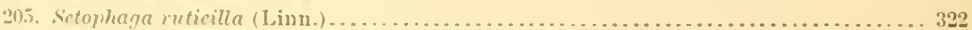

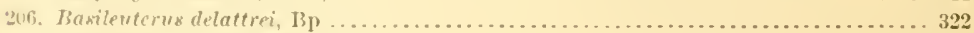

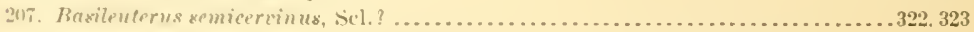

TIREONID.E.

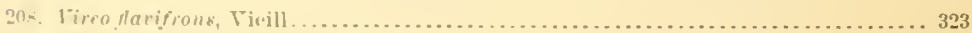

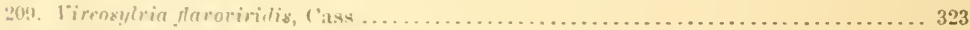

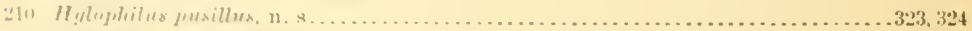

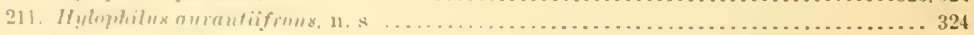

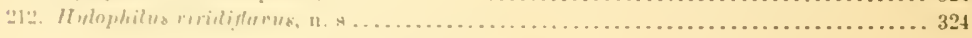

F(1).11C.1111).

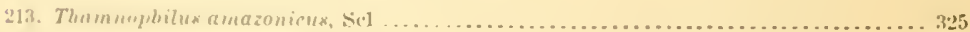

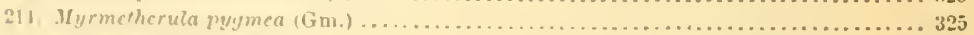

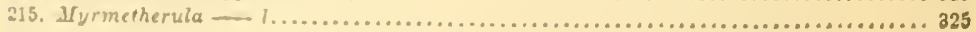




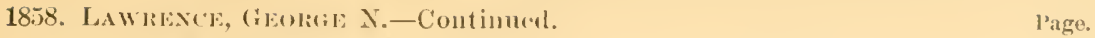

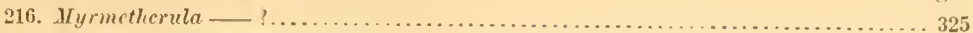

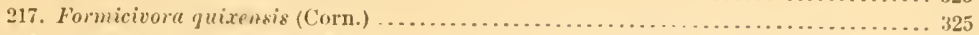

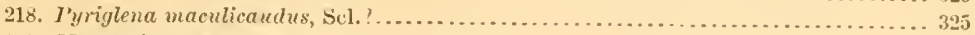

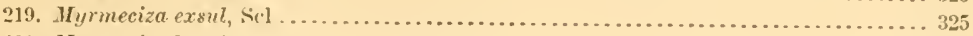

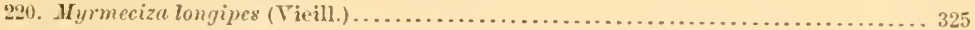

221. Iypocnemis schistacea, sel . . . . . . . . . . . . . . . . . . . . . . . . . . . . 325

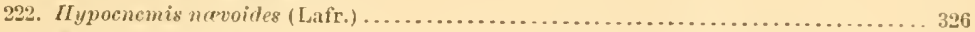

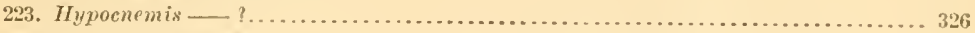

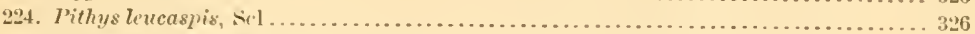

225. Formicarius analis (D'Orb. et Lafr.) .................................... 326

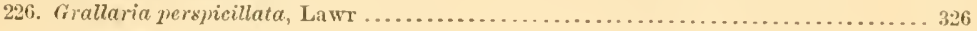

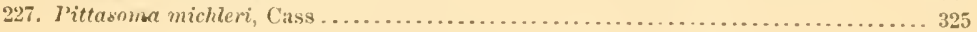

\section{TERDIDE.}

228. Turdus fucsecens, Steph . . . . . . . . . . . . . . . . . . . . . . . . . . . 326

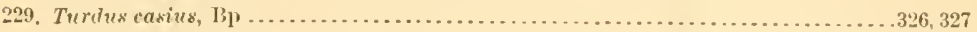

TYRANNIDE.

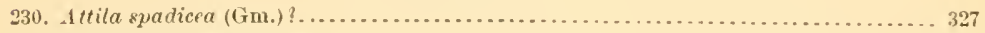

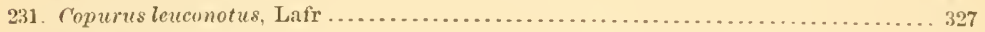

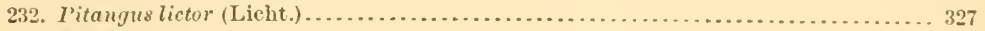

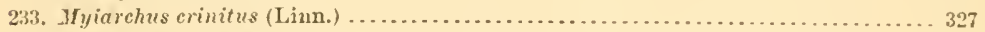

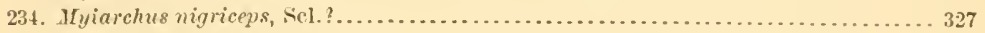

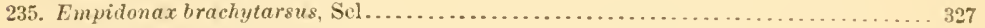

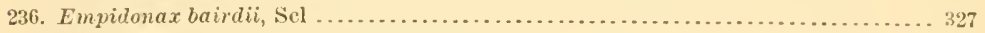

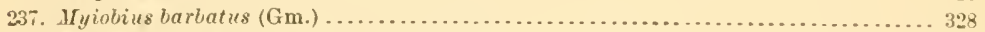

238. Myiobius cinnamomeus (Lafr. et D'Orb.) ?............................... 328

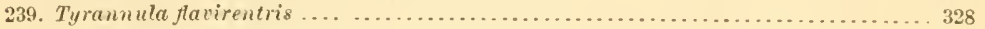

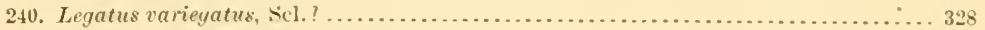

241. Myiozctetes columbianus, Cah. \& Hein............................... 328

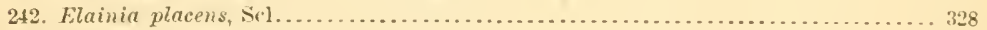

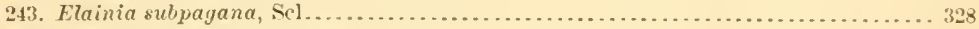

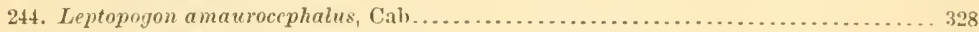

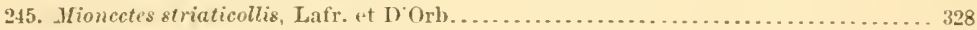

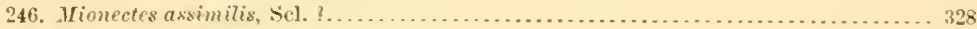

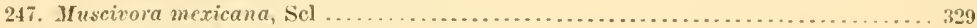

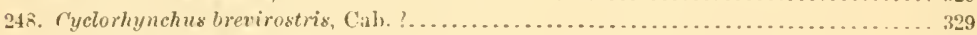

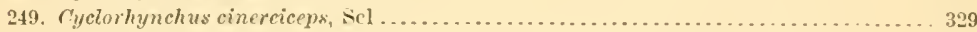

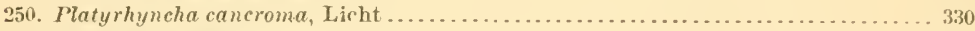

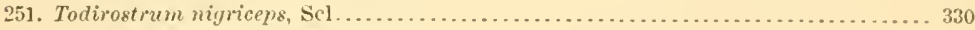

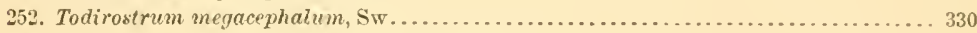

COTINGIDE.

253. Pachyrhamphus marginatus (Licht.) ?............................. 330

254. Pachyrhamphus cinereus (Borld.) ? ................................. 330

255. Lipaugus unimfus, Sel ........................................ 330

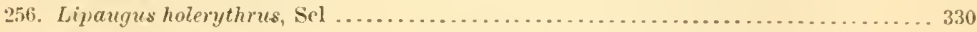

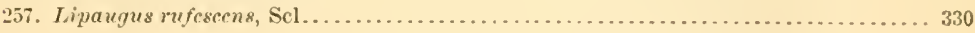

\section{ICTERDÆ.}

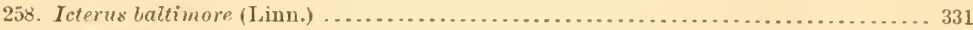

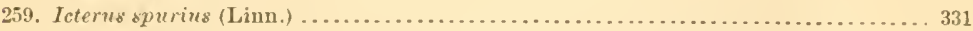

TANAGRIDE.

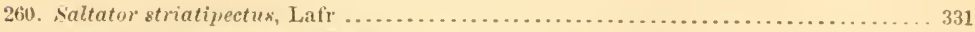

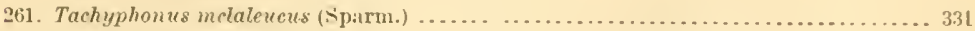

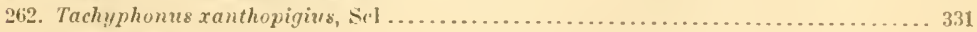

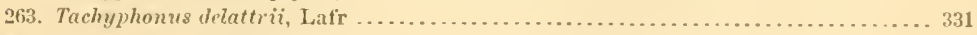

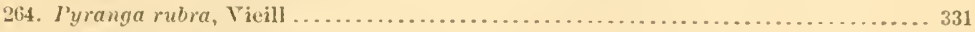

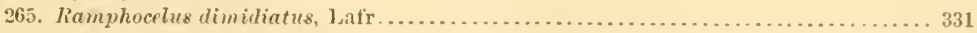

266. Ramphocelus luciana, Lafi . . . . . . . . . . . . . . . . . . . . . . . . . . . . . 331,332

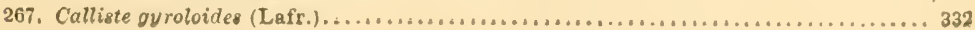




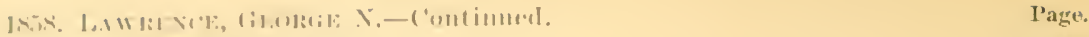

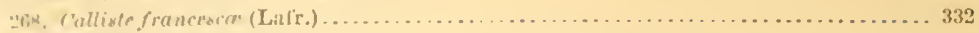

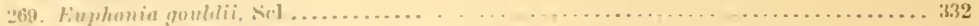

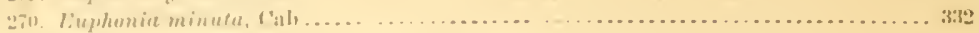

Flil Nitill,III:

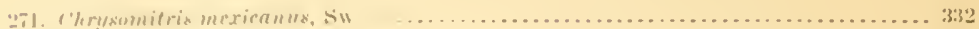

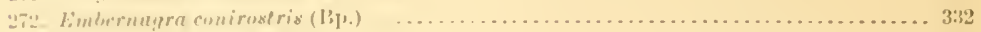

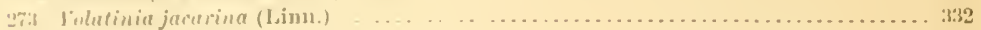

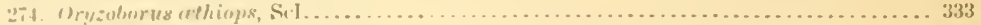

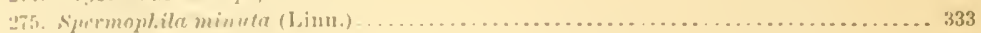

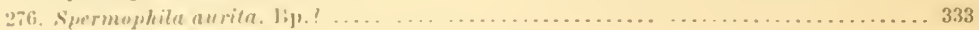

I'SIT'TCIII E

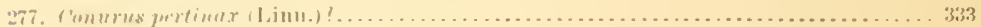

\section{JIII) E.}

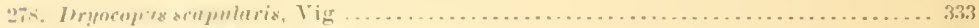

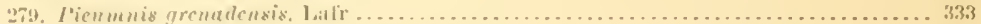

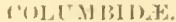

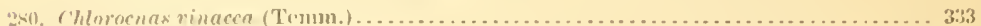

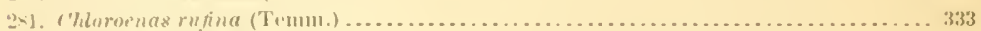

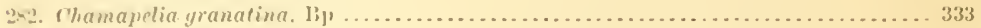

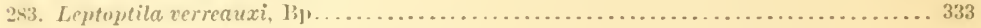

P'RACIDE.

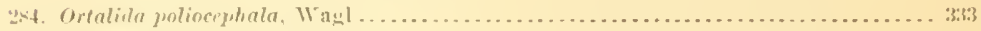

TIXAMII)

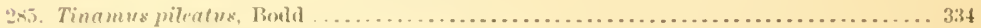

('II.IR.II)II AD.F.

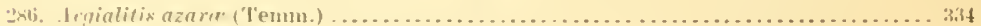

ARIHEIII.E.

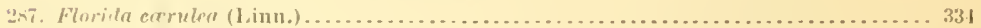

'IAX'AIIIF.

2ax Tuntalus loculatur (1,inn.) 334

ה

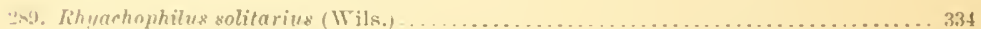

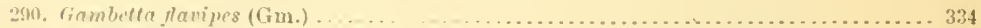

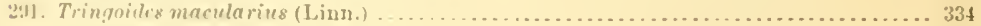

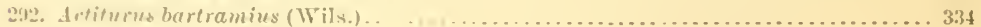

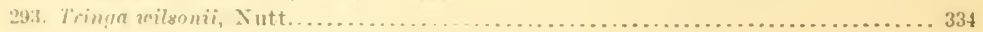

I'II, X HISII).

294. I'ndicepls dominirns (K,inu.) 334

31.

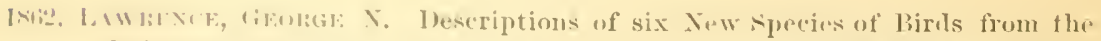

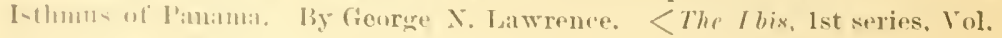

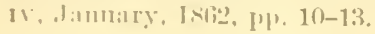


1862. DaWBNCE, (isorak N.-Continued.

TYRANNIDE.

2. I'itangus albovittutus, I' Page.

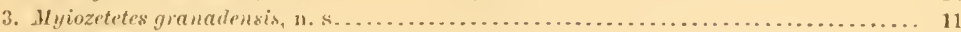

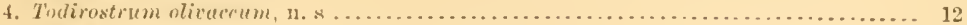

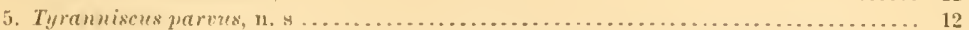

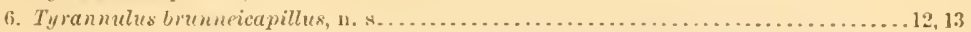

32.

1862. Jawrexce, Geobrik $\mathrm{N}$. Descriptions of Six New species of lirds of the Families Charadrida, 'Trochilidie, and Caprimulgidar. By Goorge N. Lawrence. liead Felruary 10th, 1862. <Amn. Lyc. Nat. Hist. New Fork. Vol. vit, 1862, $455-460$.

1. Aeginlitis temuirostris,

2. Thaluenia luciu,

3. Chlorostilbon insuleris, n. . ............ Tres Marias Islanils, Mexiro.......... 457

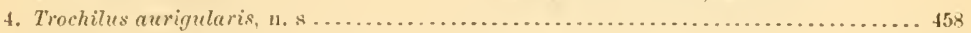

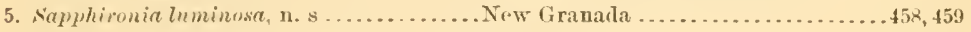

6. Stennysis maculicaudus, n. s........... Para ......................459,460

33.

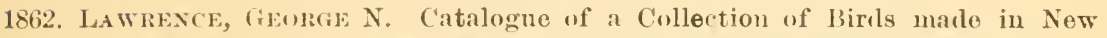
Granada. By James MeLeaman, Esı., of New York, with Notes and Deseriptions of New Species. I'art III. By George N. Lawrence. Read Felornary 10, 1862. <Ann. Lye. Nat. Hist. New Fork. Vol. v11, 1862, pp. 461-479. Contains corrections of some ascertained errors of previons parts.

\section{FALCONIDE.}

Page.

295. Thrastëtus harpyia (Liun.) .................................... 461

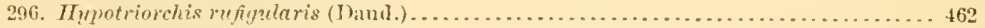

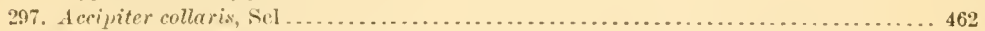

298. Micrastur scmitorquatus (Vieill.) .............................. 462

STRIGIDE.

299. Scops choliba (Virill.) ......................................... 462

300. syrnium liwatum. 11. s. ....................................... 462,463

TlOG()X11)

301. Trogon comeinums, n. s

$46: 3,464$

J)IC'ONIDE.

302. Rueco pectoralis, Cr. IR. ('say. . 464

('TREBIDE.

303. Dacnis renusta, n.s. 464,465

* Figurerd in The I bis, lstis, Pl. vir.

THO('IIILII)E.

304. Chrysureniu clicie, Boure, it Muls 465

3015. Lophornis delattrei. Jess .

\section{I)ENIPOCOLAPTIDE.}

30fi. Selemms mexicanus, Srl.?.

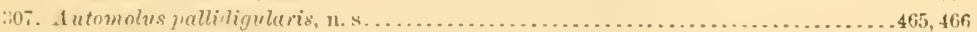

308. Dendromanes homochrous, Sil . . . . . . . . . . . . . . . . . . . . . . . . . . 460

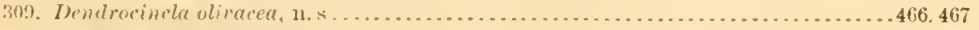

310. Dendrom is lethrymosux, и. . .................................. 467

Bull. 4(), N. MI._- ' 


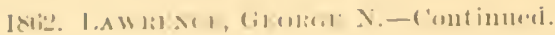

Tl(K)L(1I)YTII)

31. Cyghorintes ghilumela sills.

M.TITI,TH.F.

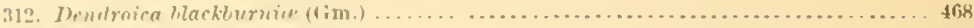

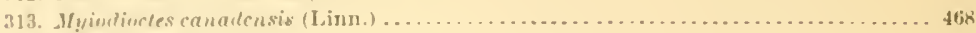

314. Operomis formosus (Wils.) . . . . . . . . . . . . . . . . . . . . . . . . . . 468

\section{VIRENIHE.}

315. Virenlaviws gulchellus, incl.

468

\section{IUIRMICARIDE.}

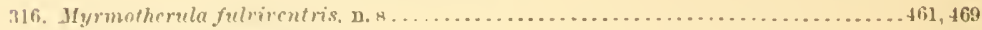

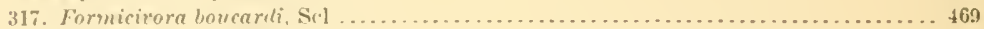

318. Kamphncanus semitorynatus. n. s............................... 469

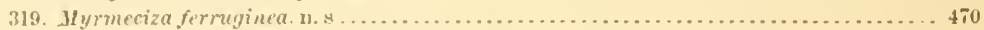

'1'1 RIIII.

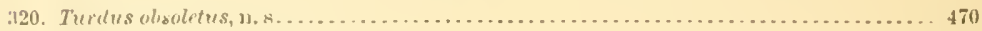

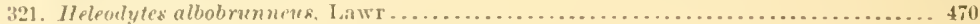

TYLANIIDE.

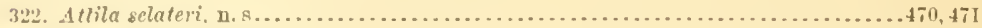

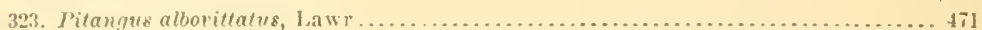

324. Empidonax grixeigularis, n. s .................................... 472

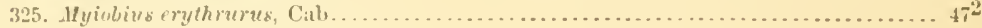

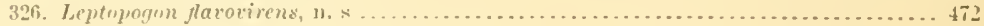

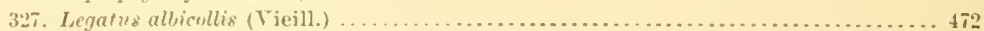

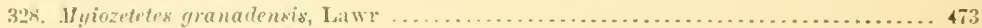

3:?. C'(emptoroma imlerbe, Sil...................................... 47

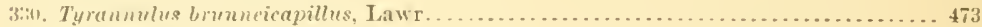

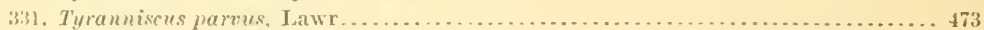

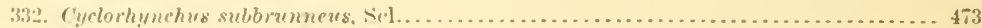

333. Cychorhynchus arquinoctialis, sel. ................................ 473

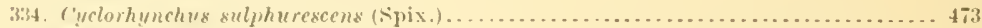

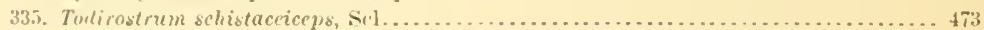

336. Oneostoma olivacea, Lawr ........................................... 47

CUTINGIINA,

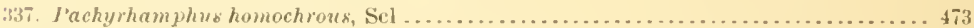

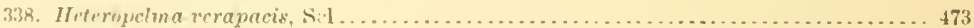

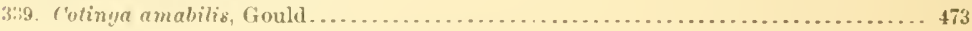

FIIXI:II,LII),

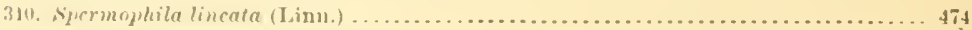

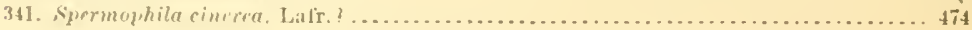

\section{RAMIUASTII)E.}

442. Nelenidera spectabilis, Cass.

\section{PSITTACID.F}

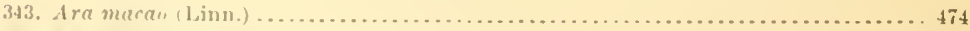

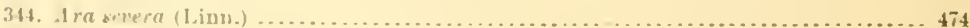

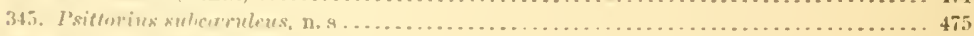

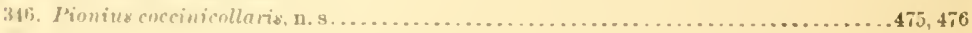

I'I('II).F,

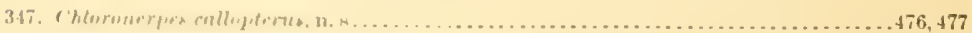


1862. LA Wrence, GEORGi N.-Contimued.

CICUI,II), E.

348. Cocengus erythrophthalmus (Wils.)

(YULUMIIDF.

349. Geotrygon violacea (Temm.)?

TINAMIII.

350. Tinamus robustus, sel 477,478

\section{('HARADRIDF.}

351. Algialitis vociferus (Limn.) 478

ARUEIDAF:

352. Botaurux lentiginosus, stepl. 478

C.ANCROMII)E.

353. Cancroma cochlearia, Linn

EURIPYGIDA.

354. Euripyga major, Hartl $4 i x .479$

TANTTALIDE.

355. Harpiprion cayennensi: (Gm.)

\section{SCOLOI'ACIDL.}

356. Macrorhamphus grizeus (Gm.) ................................. 479

357. Gambetta melanoleuca (Gm.) . . . . . . . . . . . . . . . . . . . . . . . . . . . 479

RALLIHAS.

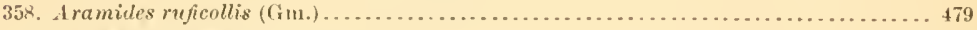

359. I'orzana carolina (Linu.) ........................................ 479

STERTIDE.

360. Hydrochelidon plumbea (Wils.) 479

34.

1863. Lawnesch, Geonge N. Deseriptions of Eight New Species of Birds trom the Isthums of Panama. By Geo. N. Lawrence. <The I bis. 1st series, Vol. v, April, 1863, pp. 181-184.

\section{HIRTXIXIDE.}

Page.

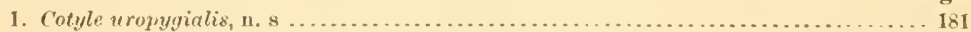

DENDROCOLA I'TIJ.T.

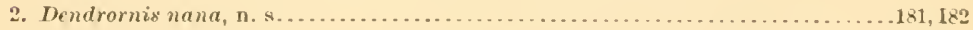

HORAICARIIIIL.

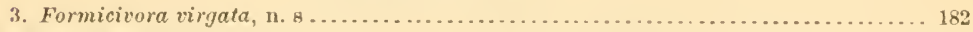

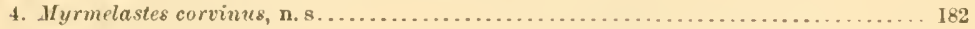

TIRANXIIA.

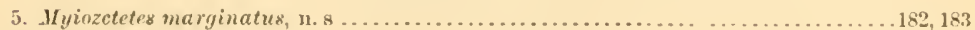

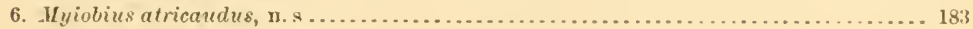

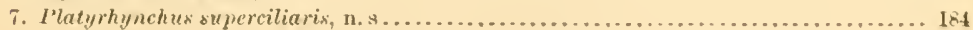




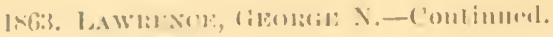

J'I('11). E.

I'ige.

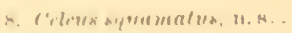

35.

18ï3. I A

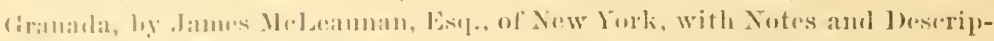

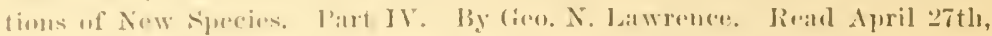

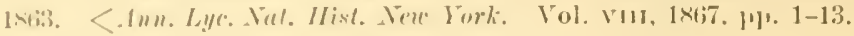

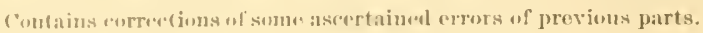

\section{VTLTTRIITE.}

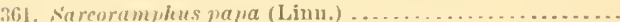

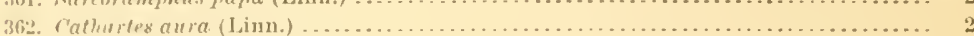

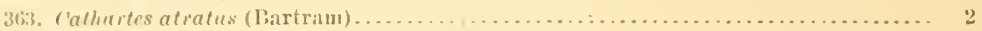

FALCONI1). E.

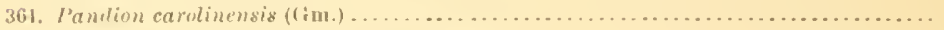

IIIRTNIIIIT.

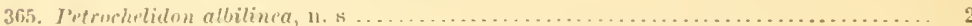

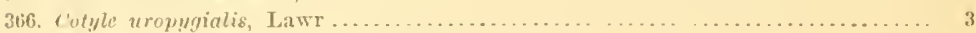

TROG(INII) $\mathrm{F}$.

367. Trogon tenellus, rah.

BTCCONII).T.

368. Malasoptila inermata (1) Bus).

TROCILILID.E.

369. Helimaster selatri, Cith.

IENIMROCOLA I'THDE.

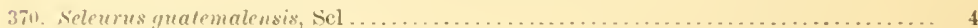

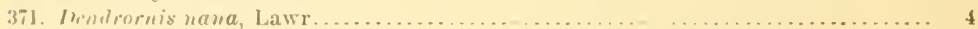

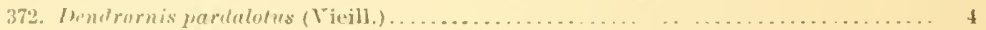

TI() )

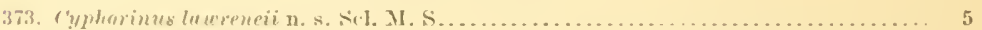

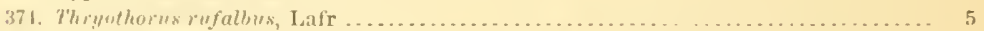

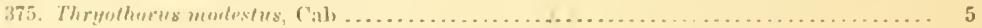

\section{MNIOTITIJ.T.}

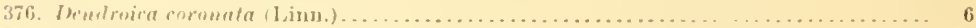

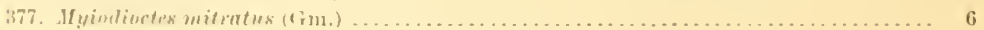

\section{VIREONIIAE.}

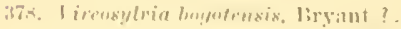

F(I:MISARIII.

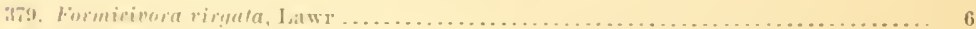

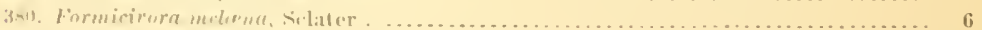

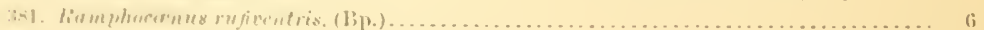

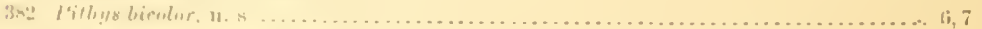

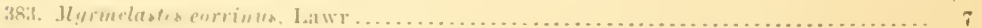

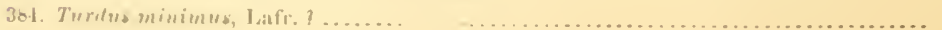


PUBLISIED WRITINGS OF GEORGE NEWHOLD LAWRENCE. 21

1863. L.AWRince, George N.-Contimmol.

TYRANNIIA.

Page-

385. Ltila citreopygius (Bom.).

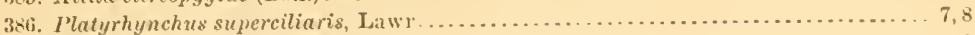

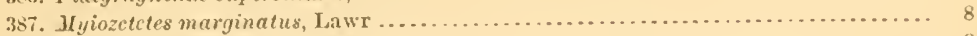

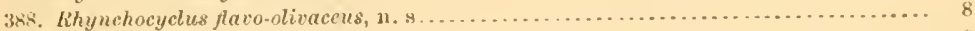

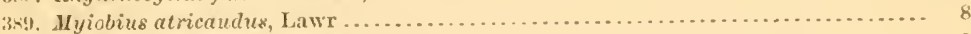

391. Myiobius sulphureipygius, sicl .................................. 8

391. Empidonax traillii (Aud.) ....................................... 8

392. Empidonax flaviventris, biairl................................... 8

\section{COTINGTHE.}

3!33. Lipaugus alboyrisem, n. $\mathrm{s}$

\section{TANAGIIDE.}

394. Fhanicothraup is fuscicaula, Cab.

FHINGILLIDE.

395. Spermophila semicollaris, n. s ................................. 10

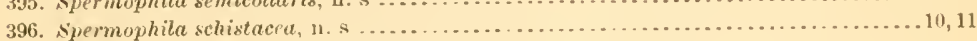

\section{PITTACIDE.}

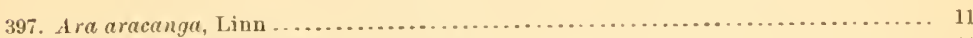

398. Conurus chrysogenys, MLass. et Sonance............................ 11

399. Prittacus pulverulcntus, Gm.................................. 11

PICIDE.

400. Celeus squamatus, Lawr

\section{CUCULIDE.}

401. Crotophaga sulcirostris, $\mathrm{Sw}$

\section{COLINIBIUE.}

402. Streptopelia risoria (Limn.)

CRACI1).E.

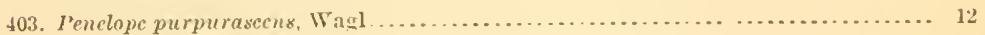

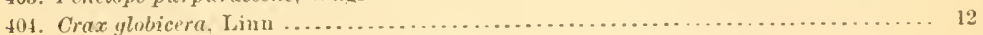

\section{ARIOEID.E.}

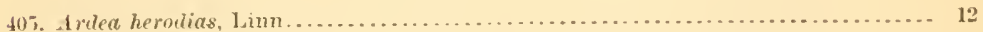

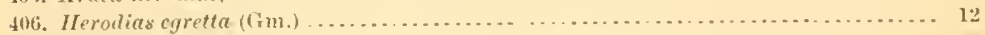

407. Ardetu cxilis (Gm.). ............................................ 12

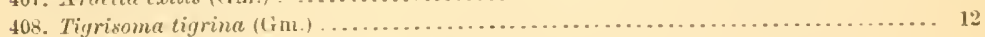

\section{$\therefore(\because 1)$ (H) ICII) F}

409. Himantopus nigricollis, Vivill ................................ 12

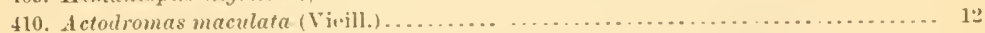

\section{INITIIE.}

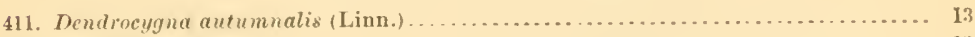

412. Anas bosehas, Linn ............................................. 13

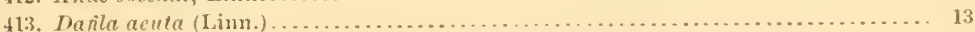

414. Cairina moschata (Linn.) ...................................... 13

PEI.EC.INIIIE.

415. Prlecanus fuscus, linn 
36.

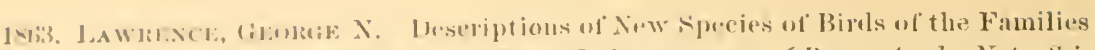

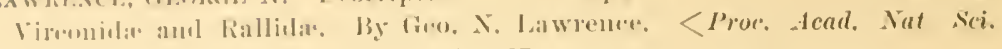
l'hilu. Vol. A 1, spril, 18ti3, pu. 106, 107.

VIREINII)E.

Yagre.

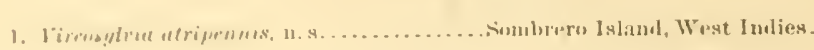
106

\section{RALLIII:E.}

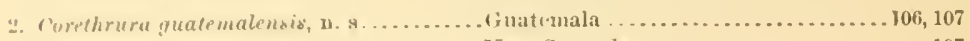

3. Aramides axillaris, n. s ............... New Granada .................. 107

\section{7.}

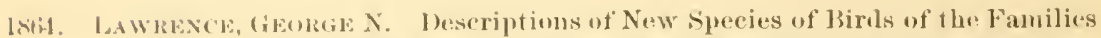

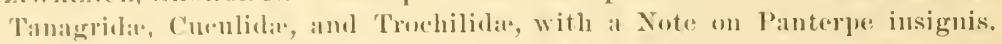
By. (Gen. N. Lawrence. Re:al .June 13th, 1864. <.Inn. Lyc. Nat. Hist. New Fork. Vul. vill, 1867, 1]. 41-16.

TANAGIII)E

Page.

1. valtator fulviventris, $11.8 \ldots \ldots \ldots \ldots \ldots .$. Paraguay .................... $\$ 1$

2. Tachuphonus tibialis, u. s...............San Jose, Costa Riea ..............41, 42

3. Tachyphonus nupensis. n. s............. Naju River, F.ualur.............. 42

CUCTLIDE.

4. ("uccyzu julieni, 11. s.................... Sumbrero Islaud, West Iudies.........42, 43

\section{TROCHILIH.F.}

5. Trochroa lencura, n. s................. Enualor......................43,44

1. I'rosticte ruficrisia, n. s............... Ecuallor ..................... 44

7. Lamphomicron olivaceus, w. s . ......... La Paz, Bolivia .................44,45

x. Panterpe insignis, Cab................ Costa Rica ................... 46

38.

1861. LAWRENC', (iEoroE N. Catalogue of Birds collected at the Island of SomIrerm, IV. I., with Ohservations by A. A. Julien. By (Beorge N. Lawrence. Re:ad llecember 12, 1864. <Aun. Lyyc. Nat. Hist. Nin Jork. Vol. vin, 1867, PI. !)-106.

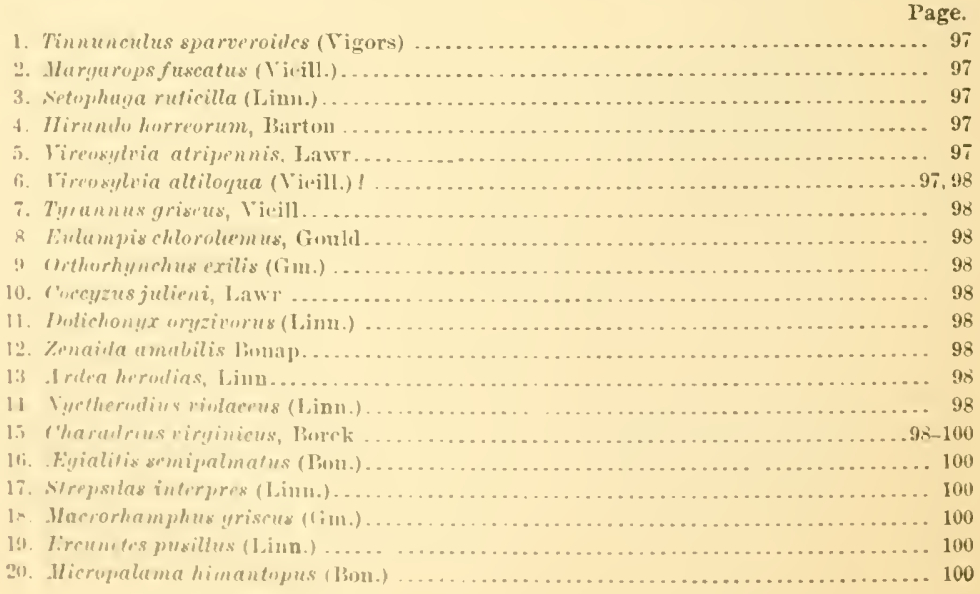




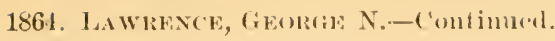

.................... 100

22. Vumenius hudsonicus, Lath:m... . . . ........................ 100

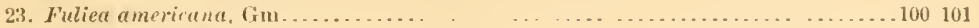

24. Querquedula tiscors (Lim.) ........................................ 101

25. Pelccanus fuscus, Limm ............................................. 101

26. Sula jiber, Linn ............................................. 101

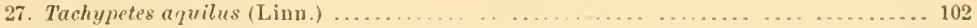

28. Phaton acthereus, Linn. ........ . . . . . . . . . . . . . . . . . . 102, 103

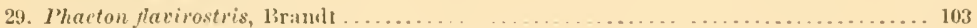

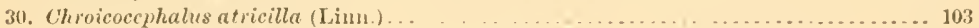

31. Thalasseus regius, Gambel......... _.. . . . . . . . . . . . . . . . 103, 104

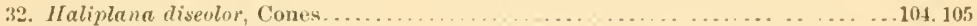

33. Anous stolirus (Linn.) .......... . . . . . . . . . . . . . . . . . . . . . . . 105, 106

34. Sterna antillarum Less........................................... 106

39.

1864. Lawkexce, Geomas $\mathrm{N}$. Deseriptions of New speries of Burts of the Families Cir rebida, Tanagrida, Irterida, and Scolopacidit. lig Geo. N. Lawrence. <Proc. Acad. Nat. śri. Phila. Vol. xvi, April, 186t, pp. 106-108.

\section{CEREBIIE}

1. Dacnis altrage.

TANA(TRII)E.

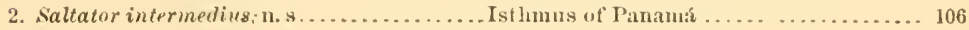

ICTERID F.

3. Cassieus vitellinus, n. s ............ 1sthnus of 1'anamá and Nicaragua .... 107

SCOLOPACIDE.

4. Erenenetes occidentalis, n. s............. Pacific coast, California ; Oregon.... 107, 108

40.

1865. Lawrence, (xEORGE $\mathrm{T}$. Descriptions of New species of Birds of the Families Tamagride Dendrocolaptidie, Fomicalide. Tyramidie, and Trochilidar By Geo. X. Lawrence. Read May 1st, $1865 .<$ Llmu, Lyc. Nat. Hist. New Sork. Tol. vill, 1867, pl]. 126-135.

TANAGRID,E.

Page.

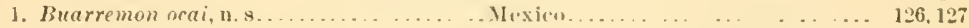

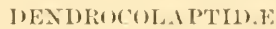

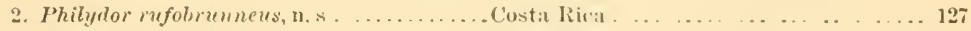

3. Inabazenops lineatus, n. \$. . . . . . . Custil Riea ... . . . . . . . . . 127, 128

4. Margarornis mbiginosa, $11.8 \ldots \ldots \ldots \ldots$. Costa lica . . . . . . . . . . . . . . . 128

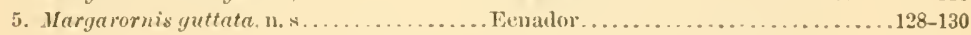

H(IRAITALII),E.

6. Dysithamnus striaticeps, n. s............ Costa lica ..................130,131

7. Dysithamuus rufeentris, n. s.......... New (ranada . . . . . . . . . . . . . 131

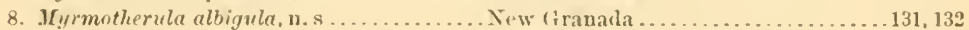

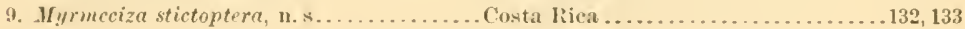

TYRANNI1):

10. Empidonax flavescens, n. . . . . . . . . Costa Rica . . . . . . . . . . . . 133

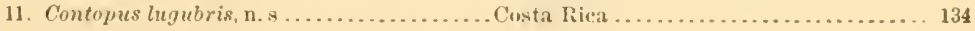


18เі) l.

\section{'Tl(1):I11.11).E}

12. Fupherasa nivercaudu, 1.,

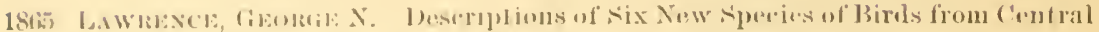

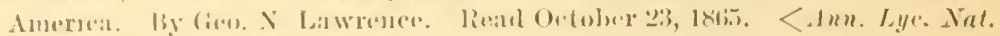

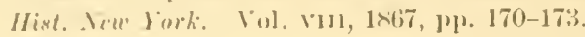

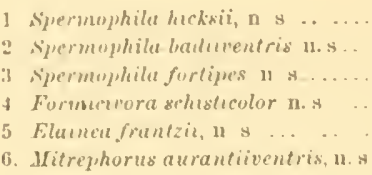

\section{2}

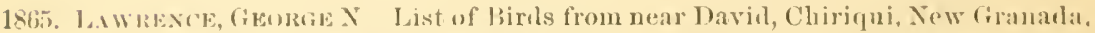
collneted for the Sunthsonian Institution, Wishington, hy Mr. Frod. Ilieks, with Descriptions of New Species. By Geo. X. Law rence. lieal Outuber 23, 1865. LAnn. Lye. Nat. Hist. Niw Fork, Vol. vill, 1867, pl, 171-178.

\section{TURDIDE.}

Page.

1. Turilus grayiz $\mathrm{Bj}$ ).

TROGLOHYTLE.

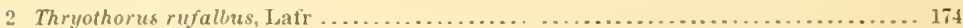

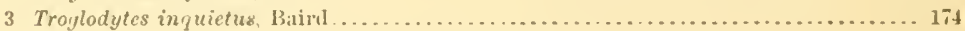

MONOTILTH.E.

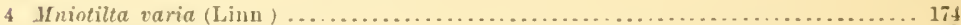

5 Melminthophaga peregrina (Wils.) ..... ............................ 1 it

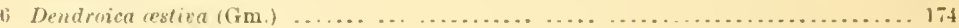

7. Setophaga ruticilla (Linn.) ..................................... 1 it

\section{C.EIEIIII.T:}

8. Chlorophanes spiza (Linu.) ................................... 174

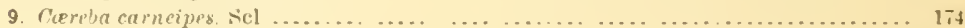

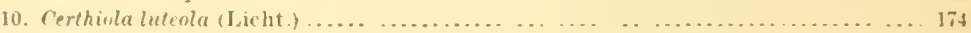

TANA(ilil1).E

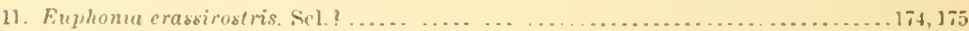

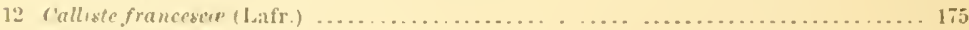

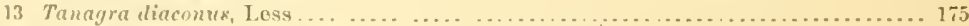

11 Ramphuselus dimidiatus, Lafr.................................. 175

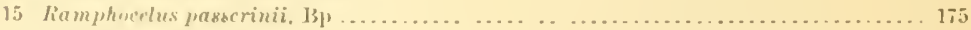

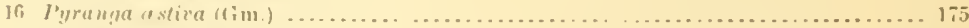

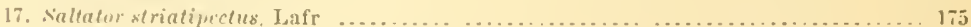

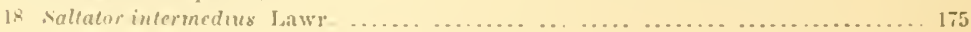

FIINTILLIDEE

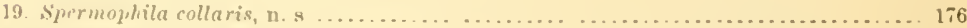

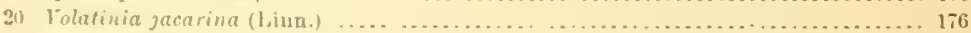

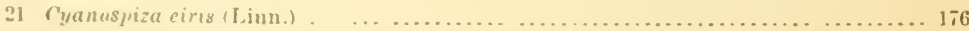

If'TElill.

22 Icterus spurius (Linn.)

2 Interes lialtimore (1,imin.)

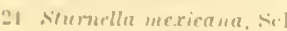


1865. LAW

TYIIAXII)E.

I’age.

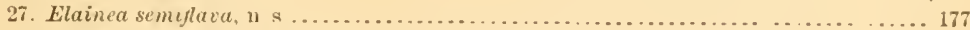

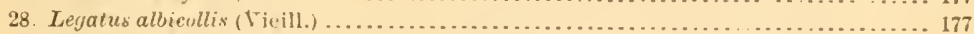

20. Hyiozetetes columbianus, Cab. et Hнin ............................. 177

30. Myiodynastes nobilis, S.1................................. 177

31. Jilvulus tyrannus (Linn.) ....................................... 177

MOMUTI1)E.

32. Homotus lessoni, Less........................................ 17

Tli()CIILID\&.

33. Lampornis veraguensis, Gould.

CICLLII)A.

34. Piaya nigricrissa, Scl .

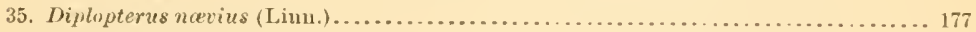

\section{RAMPHASTIUસ.}

36. I'teroglossus erythropygius, Gould 178

I'ALCONII) E.

37. Asturina magnirostris (Gm.) 178

COLUMBIDE.

38. Chamapelia nupennis, Bp. 178

RALLIDE.

39. Aramides rufieollis $(\mathrm{Gm}$.$) ?$ 173

43.

1865. Lawrexcr, Geolige X. Catalogue of a Collection of Birds in the Mnsenm of the Smithsonian Institution, mase hy Mr. H. E. Holland, at Greytown, Nicaragna, with Hescriptions of New species. By Geo. N. Lawrence. Real October 23, 186.5. <Anu. Lye. Nut. Hist. New Fork. Vol. VIn, 1867, pp. 178-184.

1. Polioptila supereiliaris, Lawr 178

\section{ILOGLODITIDA.}

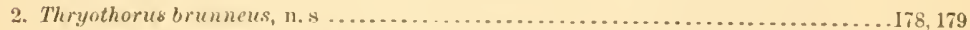

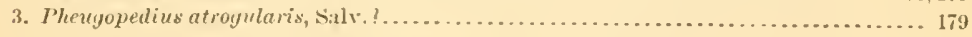

\section{MNIOTIITIDE.}

4. Henicociehla aurocapilla (Limn.)................................... 179

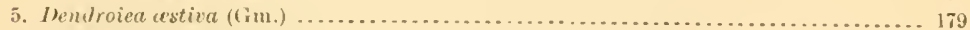

6. Basileuterus uropygialis, Srl........................................... 179

VIREONID.E.

7. Hylophilus pusillus, law 179 


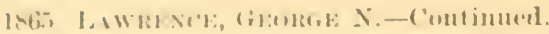

TAXAGIIINE.

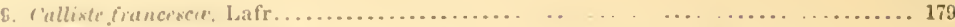

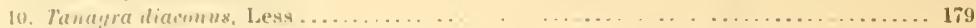

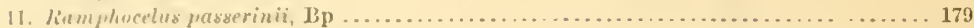

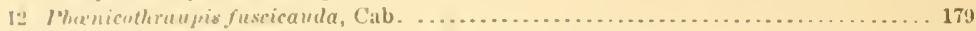

1: Irremen uuraneirnstrix, Jatir..................................... 179

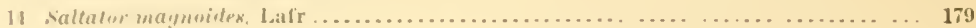

F'1RIN(i11,1,11).

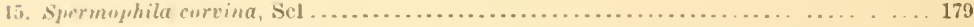

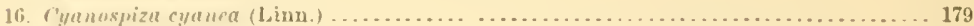

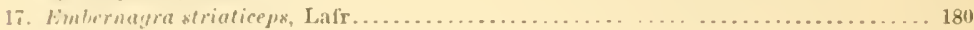

18 Enspiza amerncana (1)

\section{1(TEL11). J".}

19. Cassiculus prevosti (L.es..) ...................................... 180

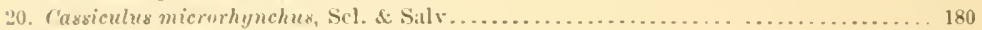

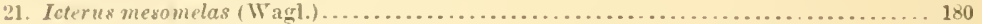

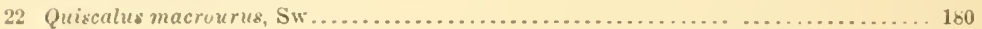

\section{1)ENDROCOLAPTII.F.}

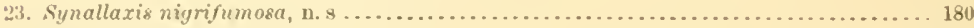

24. (ily horhynchus pectoralis, Scl. \& salv .............................. 180

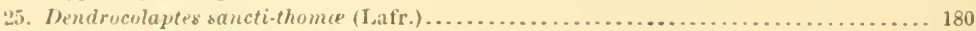

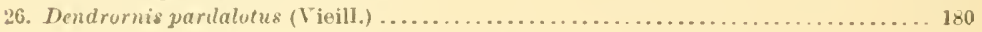

\section{FORUICARIDE.}

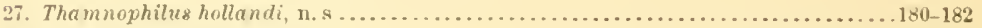

2.. Thamnophilus a finis, Cab ....................................... 182

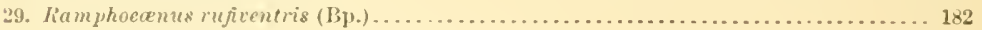

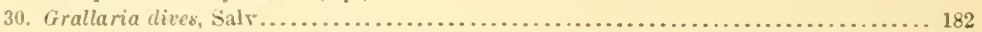

\section{TYIANNID.E.}

31. Todirostrum einereum (Linn.) ..................................... 182

32. Tyrannulus semihavus, scl. \& Saly . . . . . . . . . . . . . . . . . . . . . . . . 182

33. Elaine semiflara, Lawr ....................................... 182

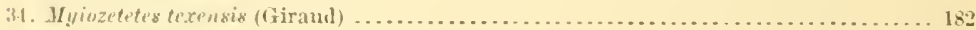

35. Megarhyuchus mexicanus (1,afr.) ................................. 182

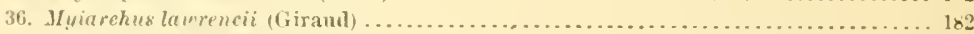

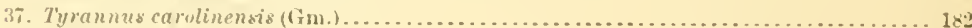

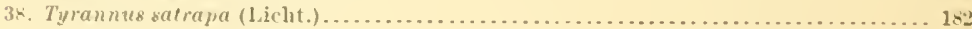

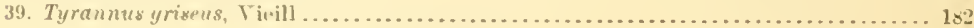

COTINGIUE.

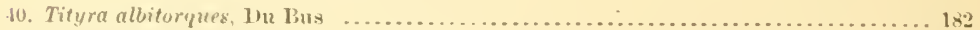

41. l'achyrhampus polychropteres (Vieill.) ? ............................... 1s?

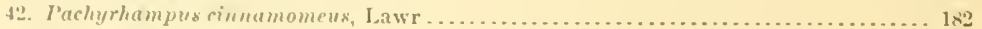

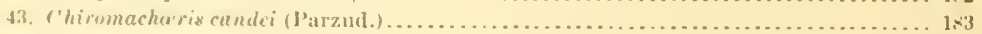

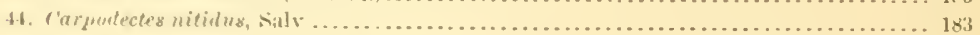

\section{ALALDINIJEE.}

15. Coryte imiln. Linun

B1\% \%ONID.F.

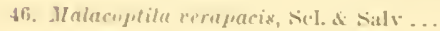

Tlumionat].

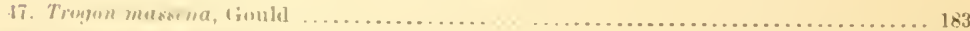

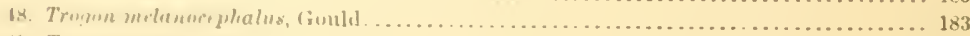

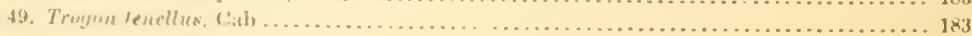


1865. Lawrexer, Groma N.-Continuel.

TROCHILIDE.

Page.

50. Pyrrhophana riefferi (Boure.)

\section{RAIPIASTIDE.}

51. Ramphastos piscivorus. Linu 183

IIIIDE.

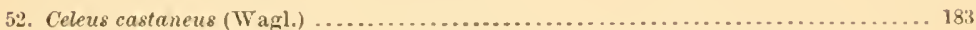

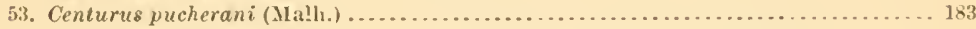

\section{PSITTACID.E.}

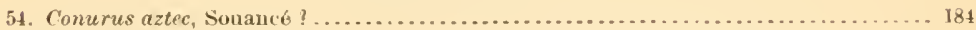

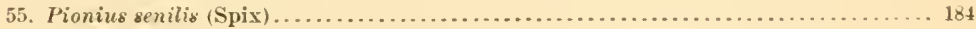

FALCONIDE.

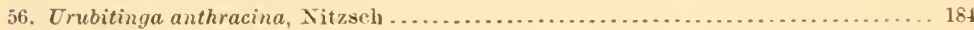

ARDEIDE.

57. Botaurus pinnatus (Licht.)?...

RALLIHA.

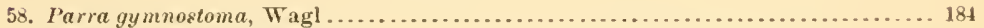

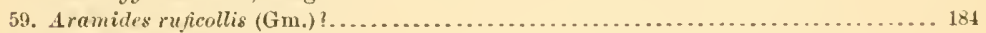

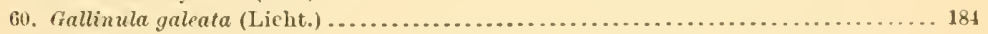

LARID正.

61. Haliplana fuliginosa (Gm.) 184

44.

186.5. Lawrence, George, N. Descriptions of new species of Birds of the Families Parida, Vireonida, Tyrannidi" and Trochilide, with a note on Mviarchus Panamensis. By Geo. N. Lawrence. SProc. Acad. Vat. Sei. Phila. Vol. xvir, Fehruary, 1865, pp. 37-39.

The note (p. 38) on M. panamensis describes a more perfect plumage tlan that of type speeimen.

1. Polioptila ylumbiceps, n.s.........

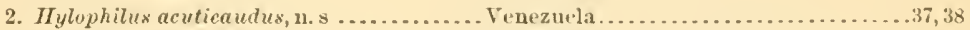

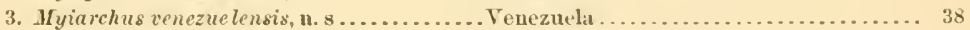

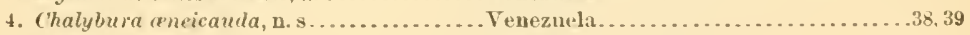

5. Chalgbura carnioli, n. s ............... Costa Rica, Angostula ............. 39

6. Panychlora parvirostris, n. s ............ Costa Riea, Angostura ............. 39

45.

1865. Lawrexce, Grokge N. Deseriptions of four new species of Birds from the lsthmus of Panama, New Granada. By Geo. X. Lawrence. <Proc. Acad. Nat. sci. Phila. Vol. xvir, June, 18tis, pp. 106-10x.

I’age.

1. Tachyphonus rubrifrons, n. s ............ Line of Pananá kailroad ........... 106

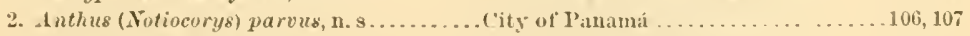

3. Thamnophilus nigricristatus, n. . ......... Line of l'anami Railroad .........107, 108

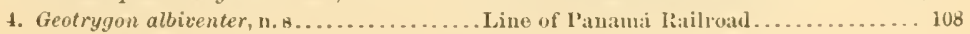


46.

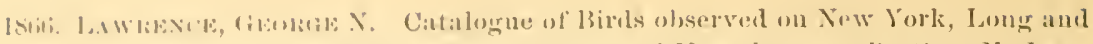

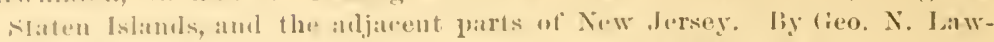

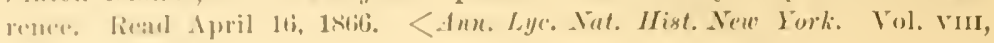
1ล(iT. ] [0. 279-300.

\section{YELTCRII.}

P’age,

1. ('athartes aura (liuu.) $27 !$

\section{FALCONID.T.}

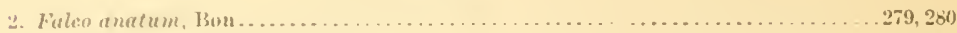

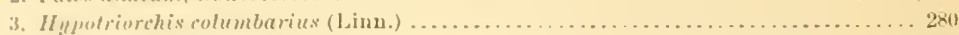

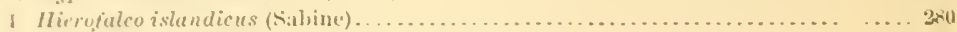

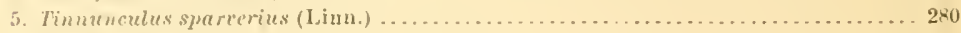

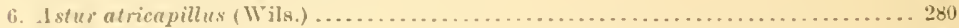

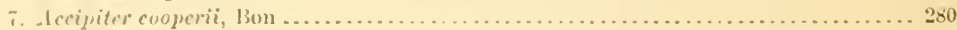

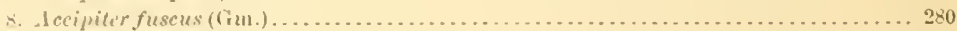

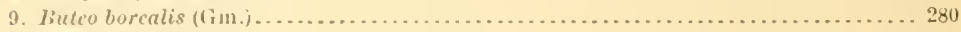

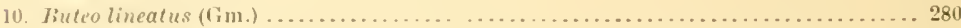

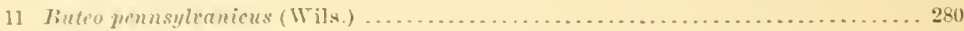

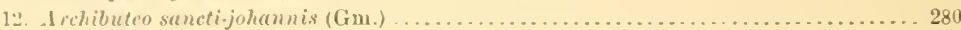

13. Vaucleves furcatus (Linu.) . ..................................... 280

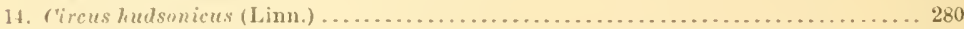

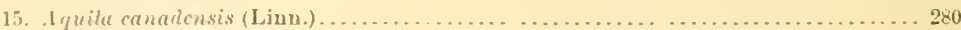

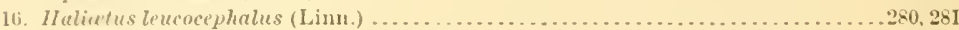

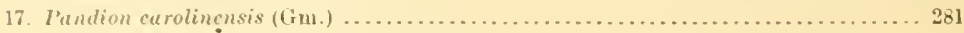

STRIGID.E.

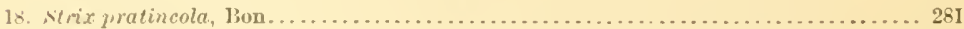

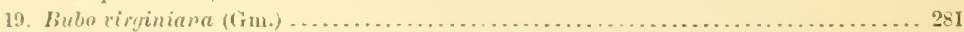

20. Neops asio (Linn.) . . . . . . . . . . . . . . . . . . . . . . . . . . . . . . . 281

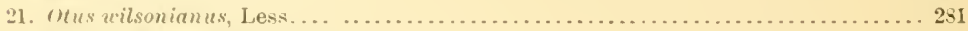

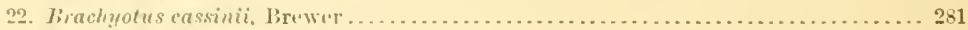

23. symium nelulosum (Forster) . . . . . . . . . . . . . . . . . . . . . . . . . . . 281

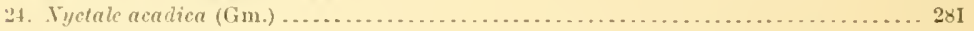

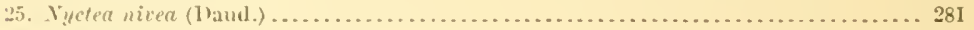

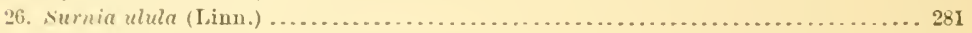

TIVIDID.E.

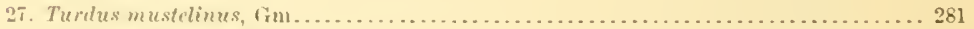

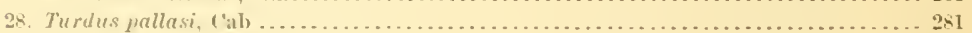

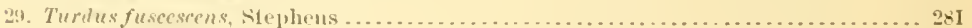

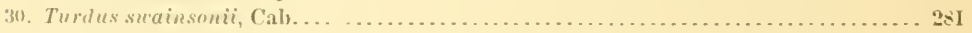

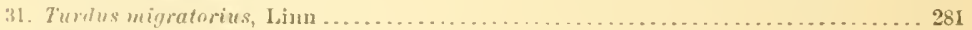

32. Turlus merius, lion ........................................... 281

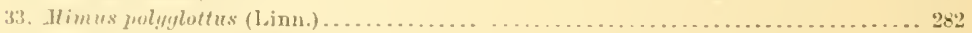

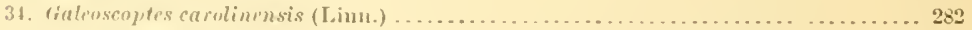

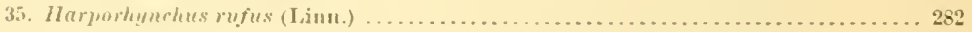

SAXICULII).

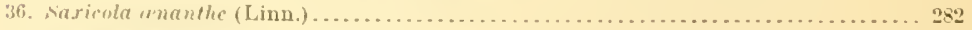

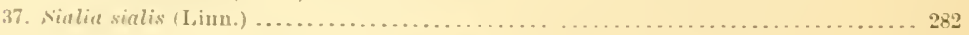

SYLVIIH.E.

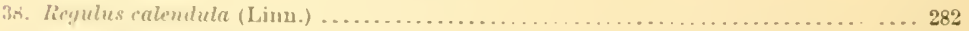

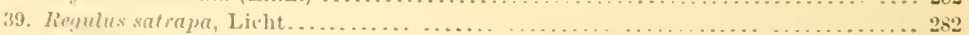

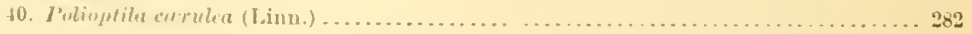

PARIII. F,

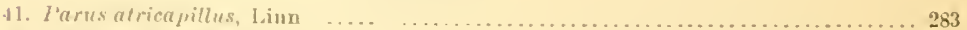

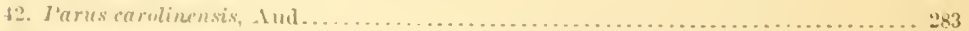

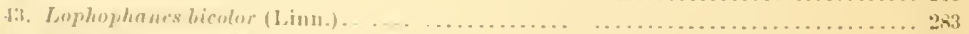

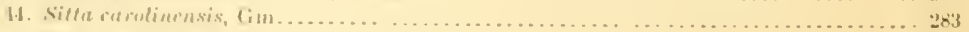

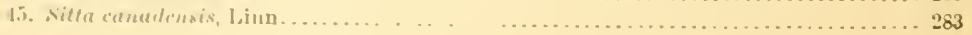


1866. LAWRENCE, GEOHEF N.-Continued.

('ERTHIADK.

46. Certhia amerioana, IBon

Page. 283

TH()(xL()I) Y'T1D.E.

47. Thryothorus ludovicianus (Gm.)

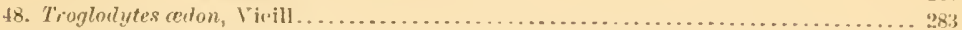

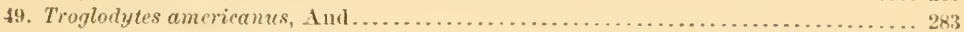

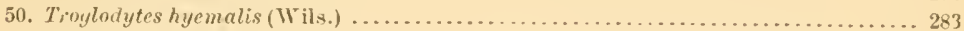

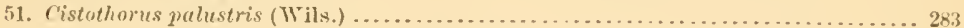

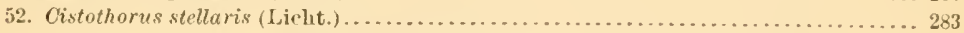

MOTACILL11).E.

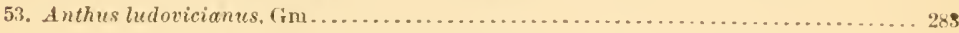

SILVICOL1WE.

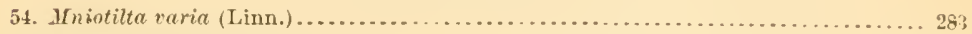

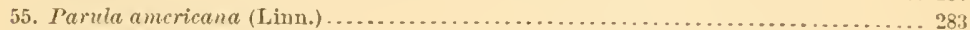

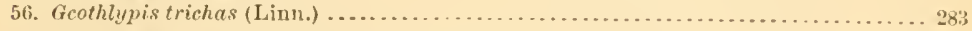

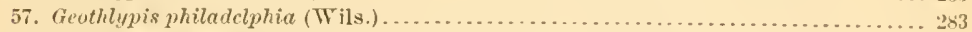

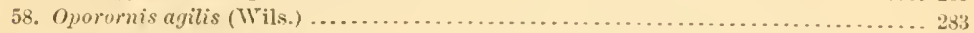

59. Oporornis formosus (Wils.) ........................................

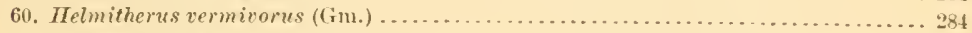

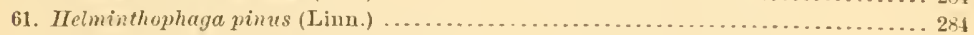

62. Helminthophaga chrysoptera (Lim.) ................................... 284

63. Helminthophaga vificapilla (Wils.) .............................. 284

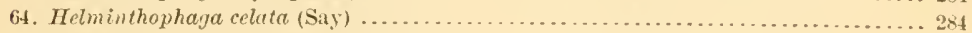

65. Helminthophaga peregrina (Tils.) .................................. $2 \times 4$

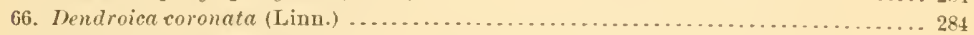

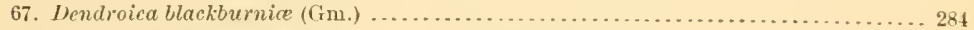

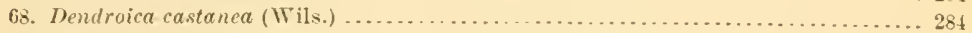

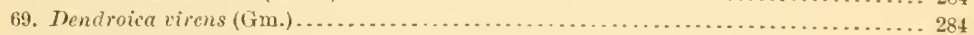

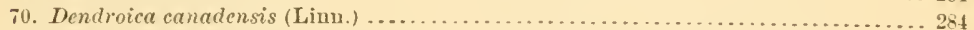

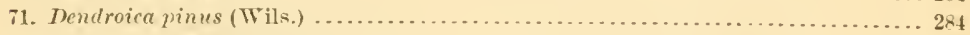

72. Dendroica pennsylvanica (Linn.) ................................... 284

73. Dendroica striata (Forster) . . . . . . . . . . . . . . . . . . . . . . . . . 284

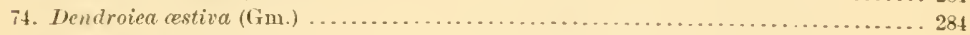

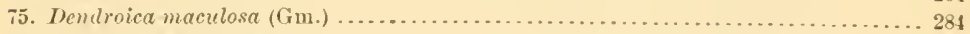

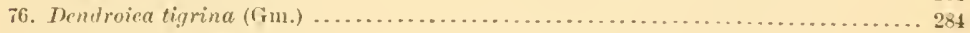

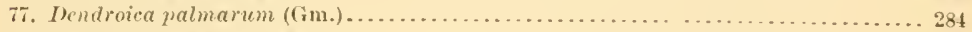

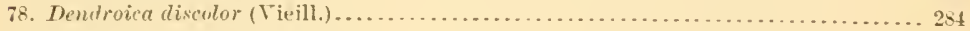

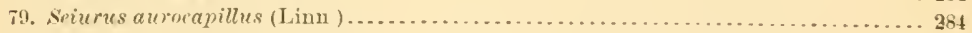

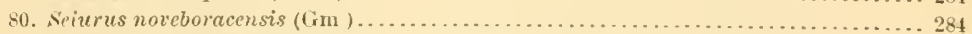

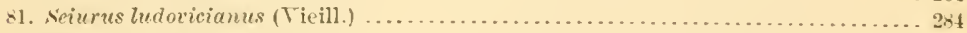

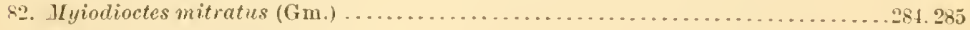

83. Myiodioctes pusillus (Wils.) .................................... 285

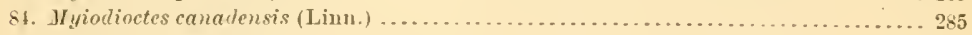

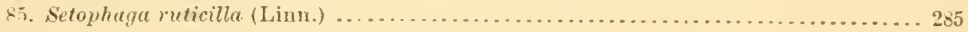

\section{IIRUNDINIDE.}

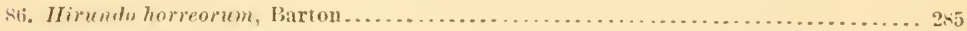

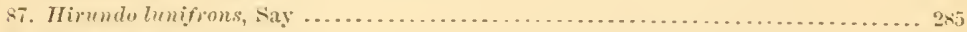

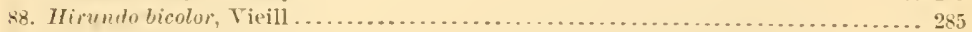

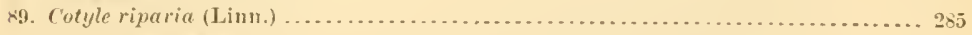

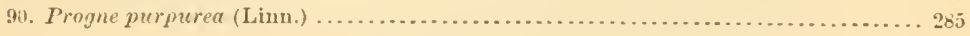

\section{VIREONHDE.}

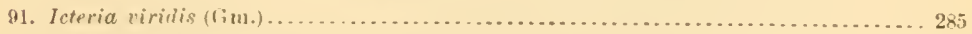

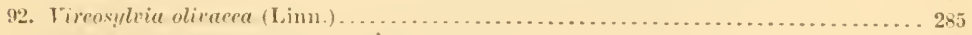

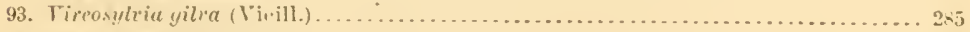

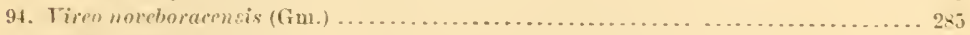

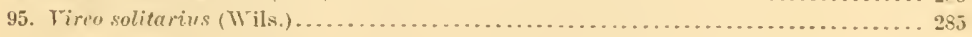

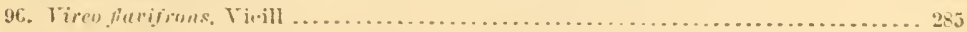


18ำ. 1.Aw

LAXIIII.E.

Page.

97. Collyrio buretatis (liwn.) ....

ANI'LL11) l:

25. Anpelis garrulus, Linn.

99. Ampclis celrorum (Vinill.).

TANATIIIDA.

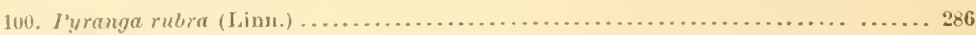

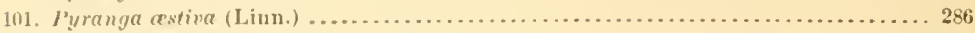

FRINGILI,IINE.

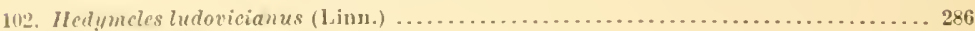

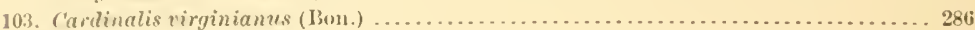

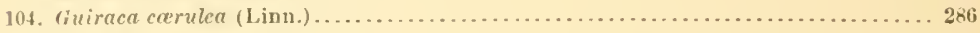

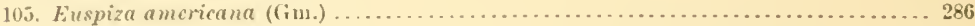

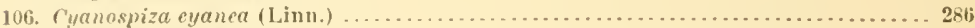

107. P'ussereulus savanиa (Wils.) . .................................... 286

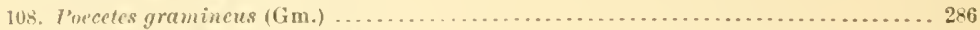

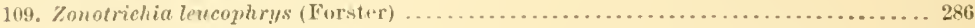

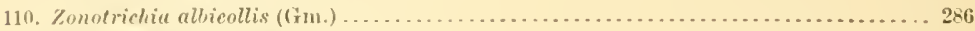

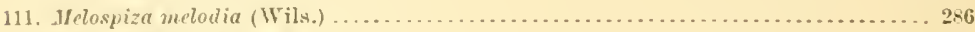

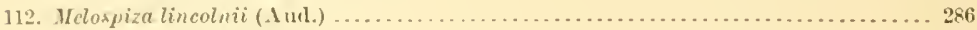

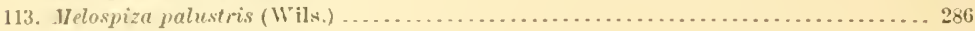

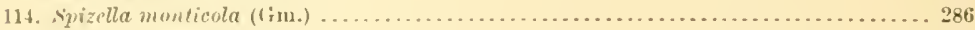

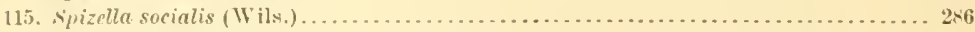

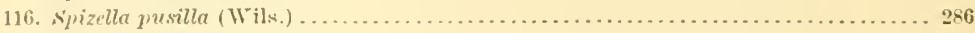

117. Junco hyemalis (Linn.) ....................................... 286

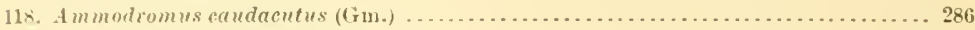

119. A mmodromus maritimus (Wils.) .................................. 286

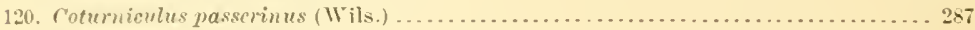

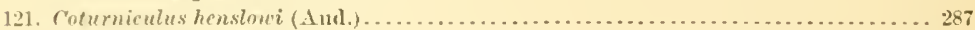

122. I'asscrellu iliaea (Merren.) . . ..................................... 287

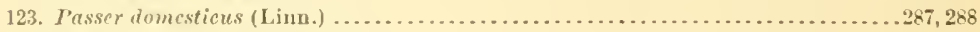

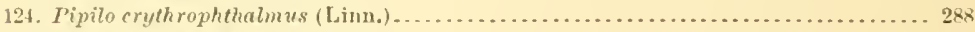

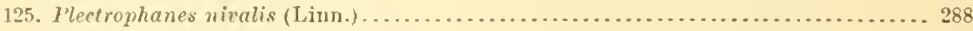

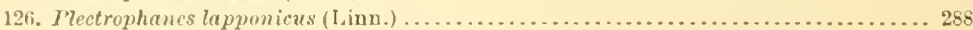

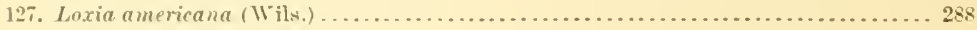

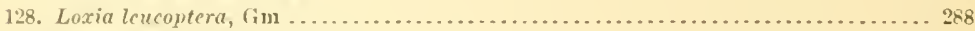

129. I'inicola canaden sis (Bris.s.) . . . . . . . . . . . . . . . . . . . . . . . . . . . . . 288

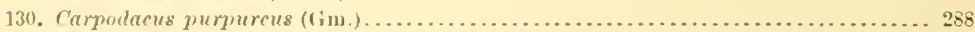

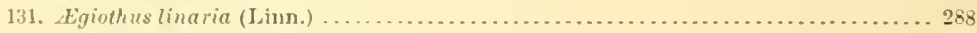

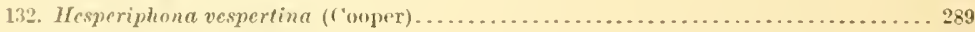

133. Chrysomitris tristis (Linn.) . . . . . . . . . . . . . . . . . . . . . . . . . . . 289

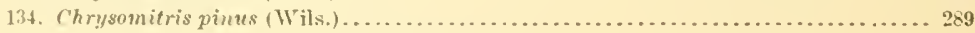

ALATIIS. E.

135. Frmophila cornuta (Wils.) .......................................... 289

16"TERIDE.

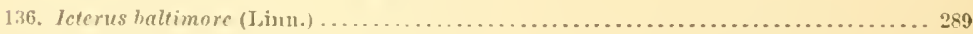

137. Ictcrus spurius (Linn.) . . . . . . . . . . . . . . . . . . . . . . . . . . . 289

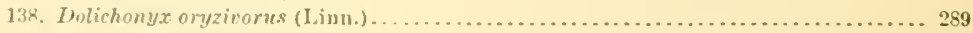

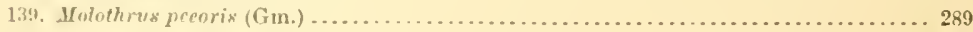

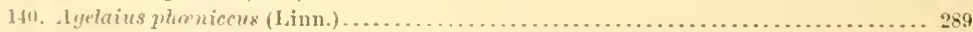

141. Sturnclla magna (Linn.) . ........................................... 289

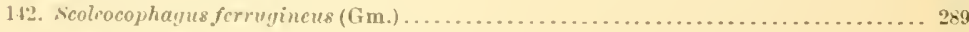

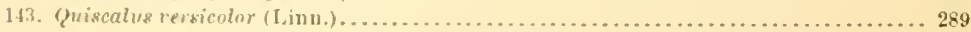

('ORVII)E.

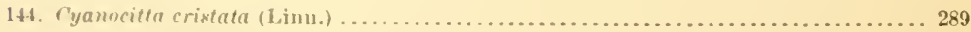

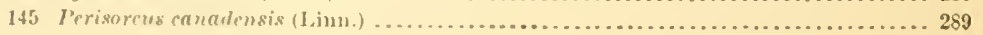


PUBLISHED WRITINGS OF GEORGE NEWBOLD LAWRENCE. 31

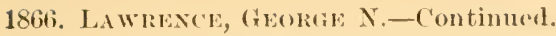

146. Corve carnivorus, Bart ram Page.

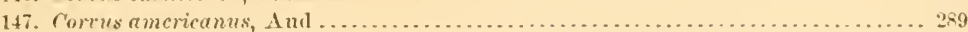

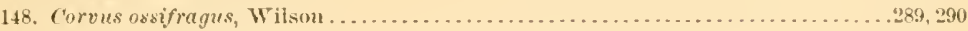

TYIRINID.F.

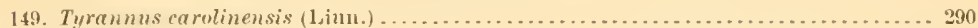

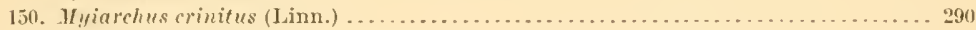

151. Empidias fuscus (1)on.) . . . . . . . . . . . . . . . . . . . . . . . . . . . . 290

152. Contopus borcalis (\$w.) . . . . . . . . . . . . . . . . . . . . . . . . . . . . . . . 290

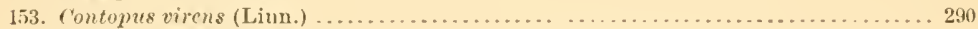

154. Empidonax traillii (And.) ......................................... 290

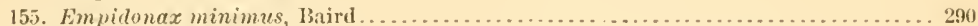

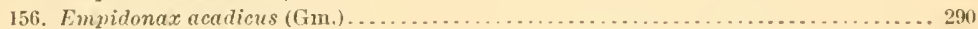

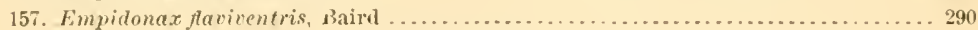

ALCEIUTIDA:

158. Ceryle alcyon (Linu.) .............................................. 290

(APRIMULGII):

159. I ntrostomus vociferus (Wits.) .................................... 290

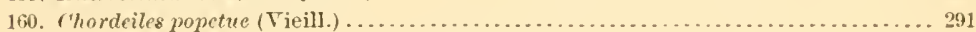

\section{CTPSELIDE.}

161. Chatura pelasyia (Linn.) 201

TROCHILIDE.

162. Trochilws colubris, Linn 291

(TICLIDE.

163. ('oceygus americanus (Linn.) 291

164. Coccygus erythrophthalmus (Wils.) 291

PICIDE.

165. Picus villosus, Linn ............................................. 291

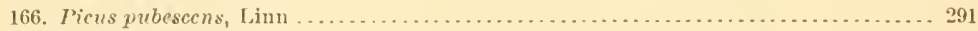

167. Picus borcalis, Vieill ........................................... 29!

168. Sphyropicus varius (Linn.) . . . . . . . . . . . . . . . . . . . . . . . . . . . 291

169. Hylotomus pilcatus (Linn.) . . . . . . . . . . . . . . . . . . . . . . . . . 291

170. Centurus carolinus (Lim.) ........................................ 291

171. Melanerpes erythrocephalus (Limn.) .................................. 291

172. Culaptes auratus (Linn.) . . . . . . . . . . . . . . . . . . . . . . . . . . . . . 291

(CLLIIBII).E.

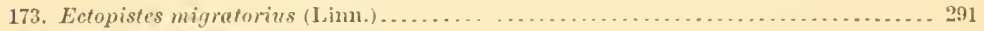

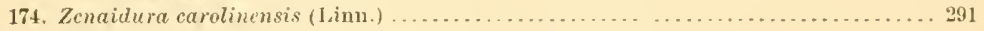

TETIR.LONII).F.

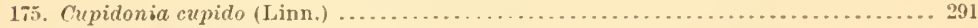

176. Bonasa umbellus (Linn.) ........................................... 291

PERDICID.E.

177. Ortyx virginianus (Linn.)

\section{ARDEIIIE.}

178. Demiegretta ludoriciana (Wiknu) ................................. 292

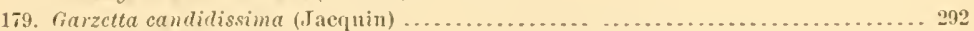

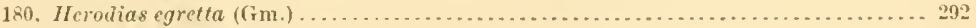

181. Irdea herodias, Linn ......................................... 292

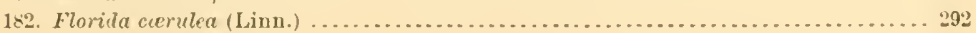




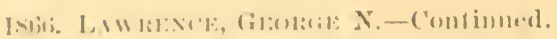

P':tge.

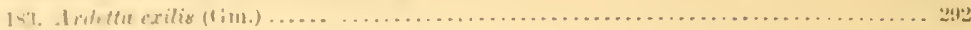

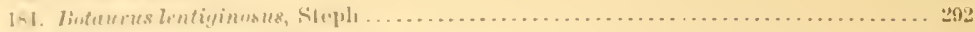

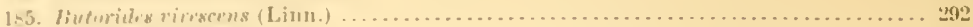

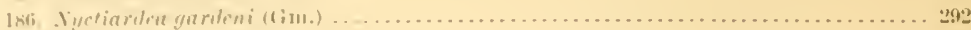

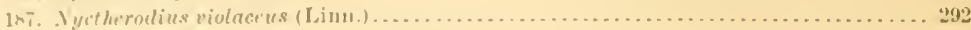

T.1NTALII).F.

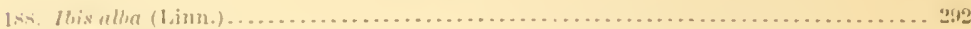

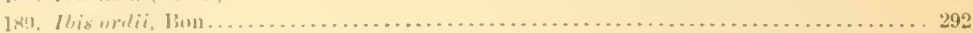

i(:OLUI'AO'IH,E.

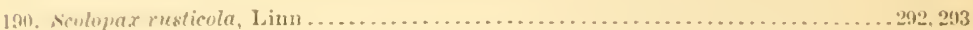

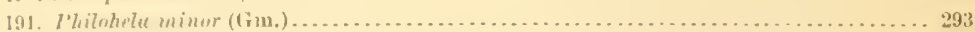

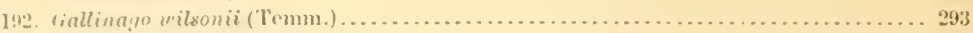

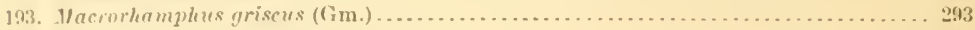

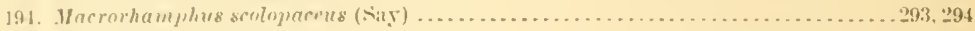

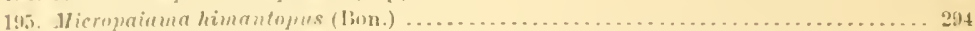

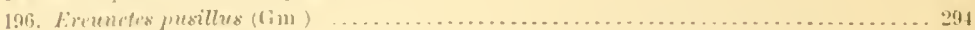

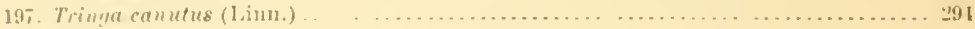

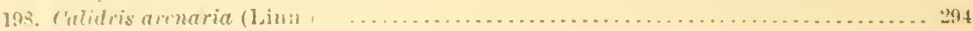

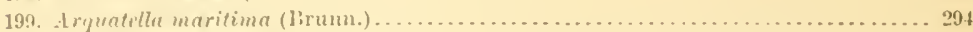

200. Aneylordeilus subarquata (Guld.) .................................. 294

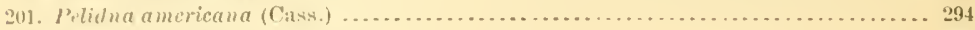

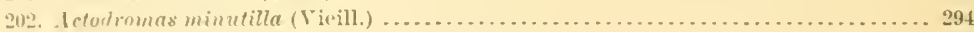

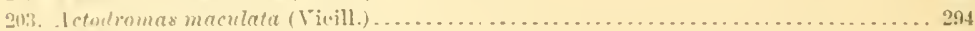

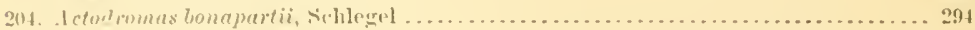

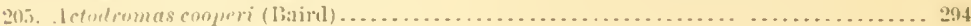

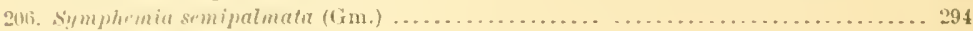

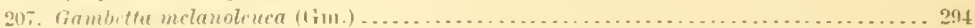

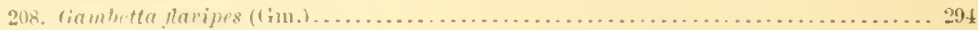

204. Rhyacophilus solitarius (Wils.) . . . . . . . . . . . . . . . . . . . . . . . 294

210. Tringoides macularins (Linn.) . . . . . . . . . . . . . . . . . . . . . . . . . . 294

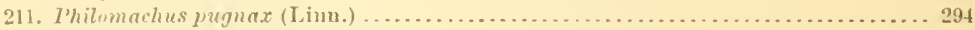

21:. Ictiturus bartiamius (Wils.) . . . . . . . . . . . . . . . . . . . . . . . . 294

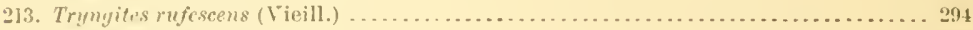

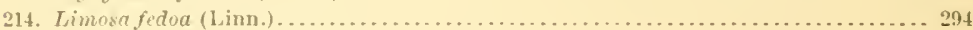

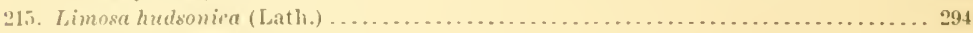

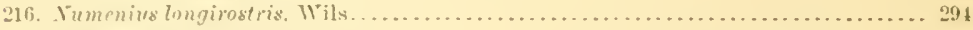

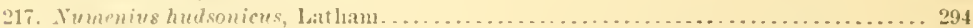

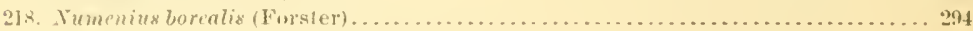

('IIAl:A1)IIII).E.

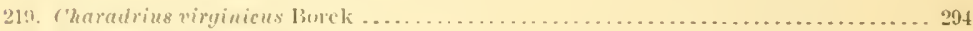

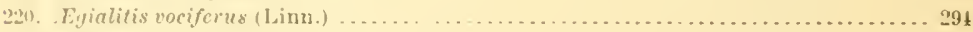

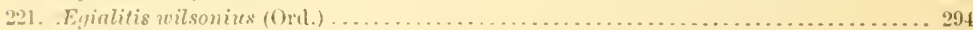

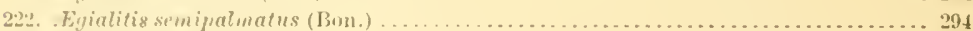

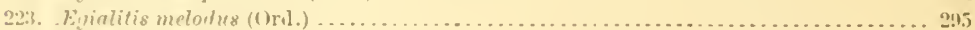

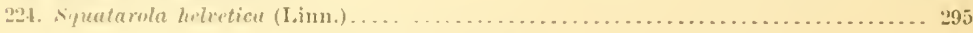

\section{II.EHATOIPIII).E.}

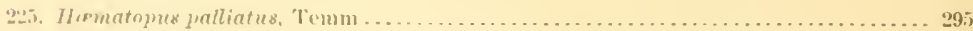

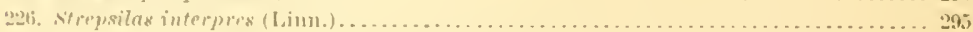

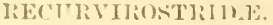

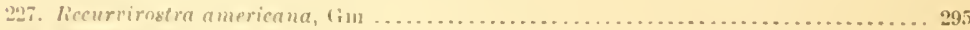

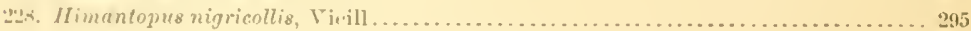

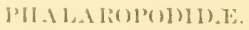

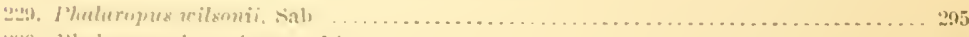

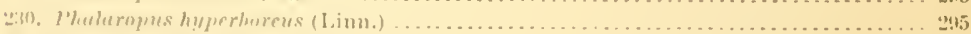

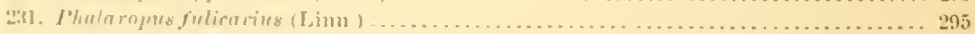


PUBLISHED WRITINGS OF GORGE NEWBOLD LAWRENCE. 33

1866. Lawhexce, George N.-Contimerl.

KILLII)E.

Page.

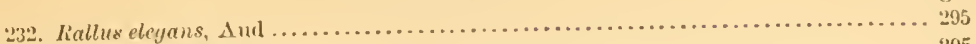

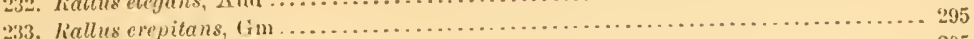

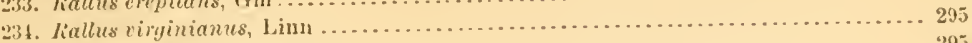

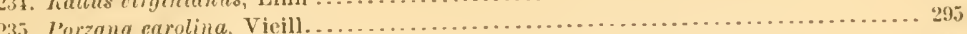

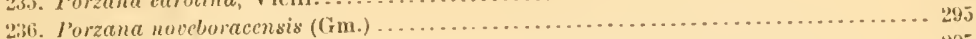

237 . Fuliea emericana, Gm ............................................. 295

238. Ciallinula guleata (Licht.) ......................................... 295

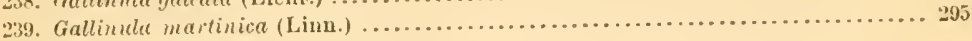

INATI1)

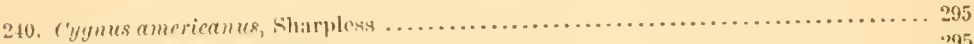

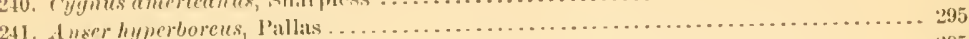

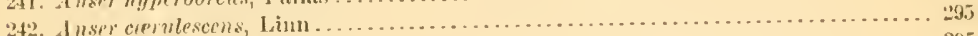

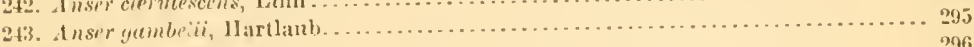

241. Berniela cand lensis (Lim.) . ...................................... 296

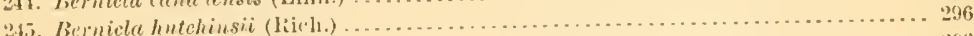

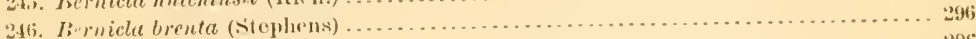

17. Jiruirla migricans (hatwr.) . . . . . . . . . . . . .

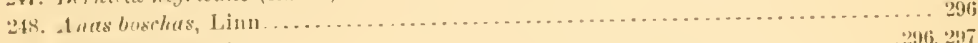

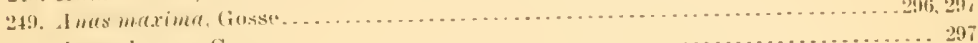

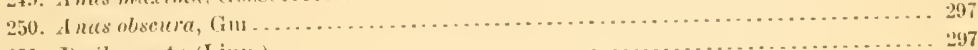

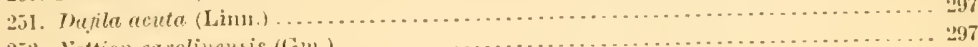

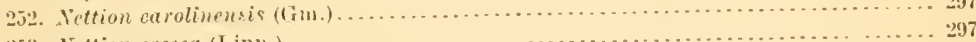

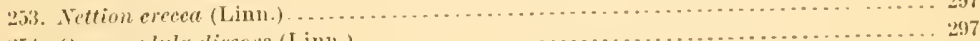

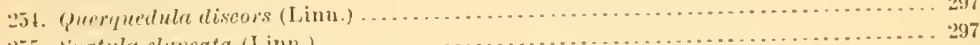

255. spatule elypeata (Linn.) . . . . . . . . . . . . . . . . . . . . . . . . . . . . . . . . . . 297

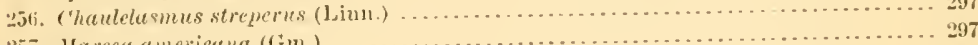

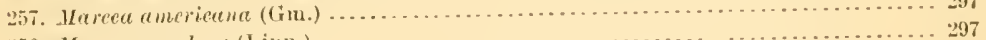

258. Harea penelope (Linn.) ........................... . . . . . . . . . . 297

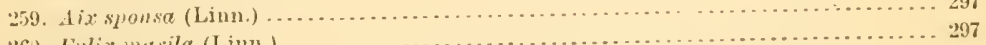

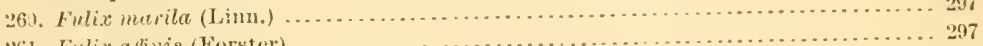

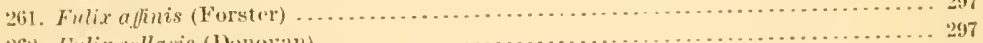

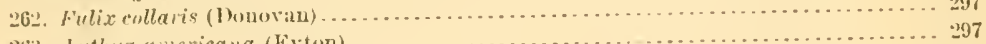

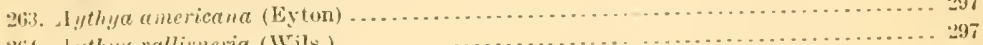

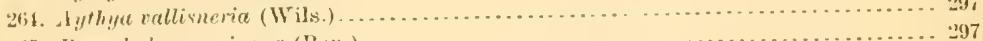

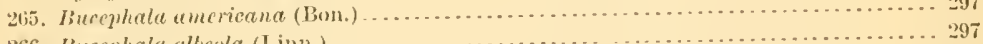

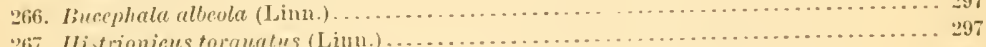

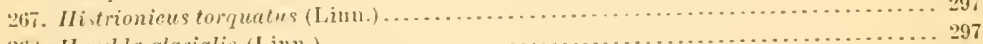

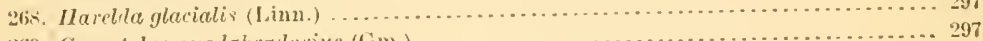

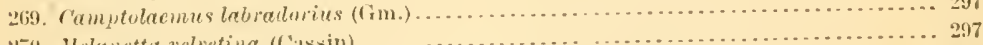

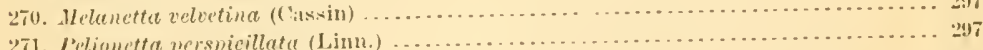

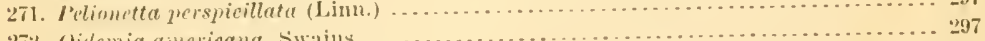

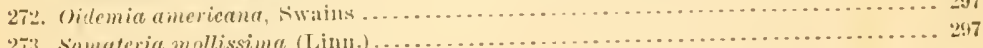

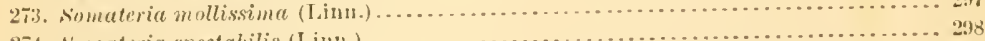

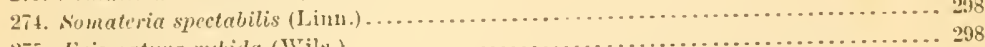

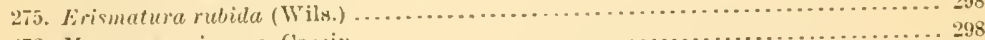

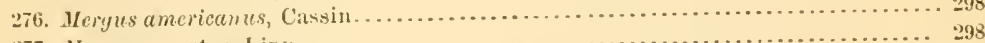

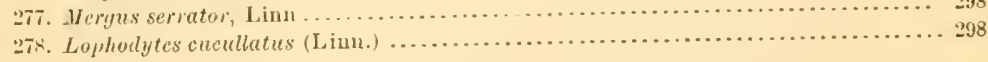

P'LLE( AXI1).F.

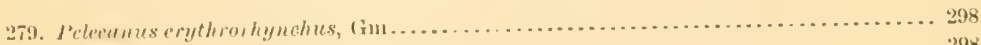

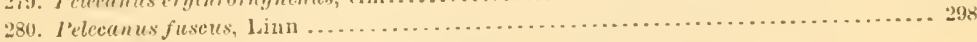

SILLII).E.

281. Sule bassena. Hris:.

PHALACHOCOHAC'HE.

28:. Graculas carbo (Linn.)

283. (iraculus dilophus (太心)

Bull. 40, N. M. - 3 


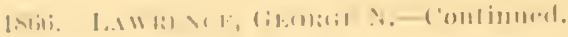

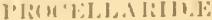

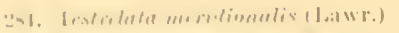

l'age.

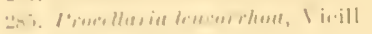

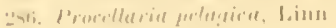

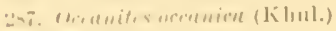

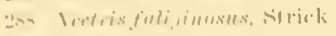

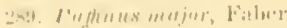

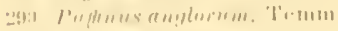

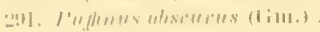

\section{T.IIIII.L.}

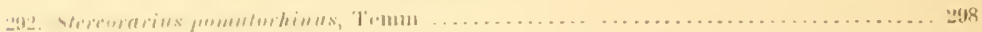

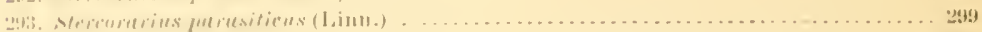

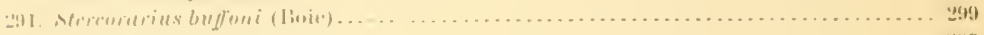

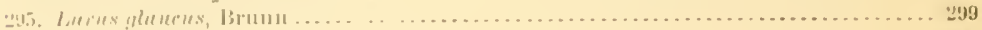

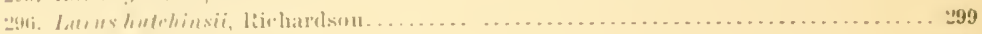

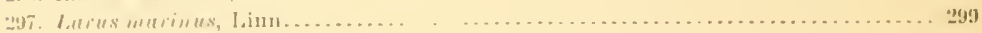

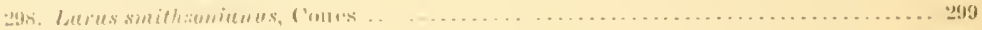

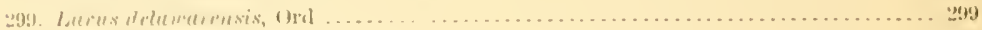

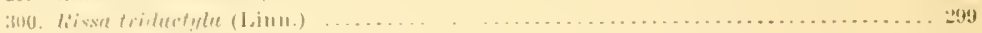

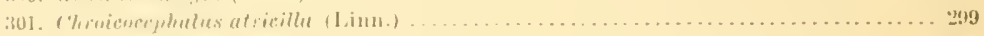

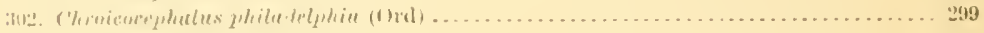

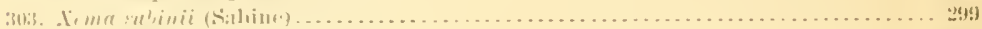

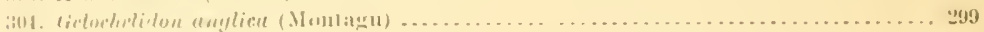

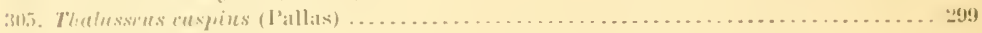

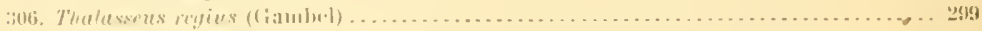

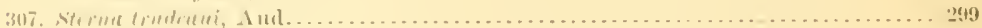

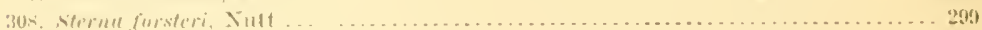

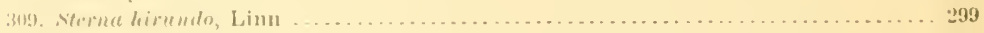

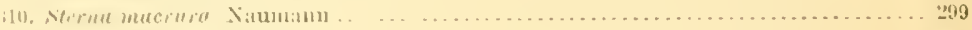

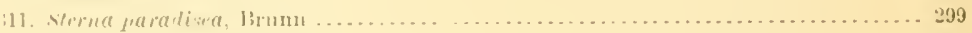

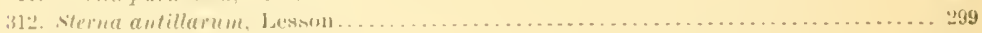

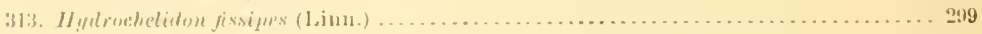

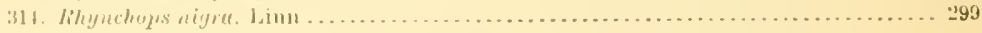

(2)I.Y III:II).E.

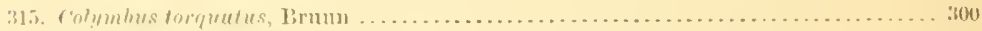

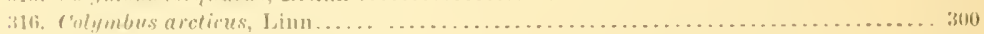

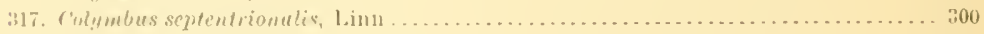

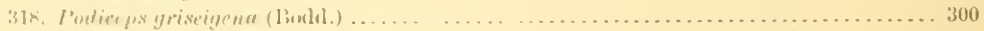

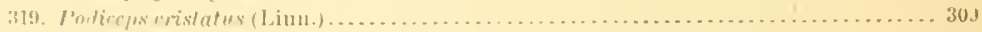

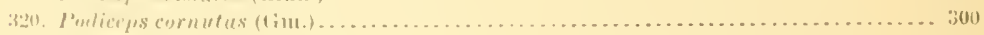

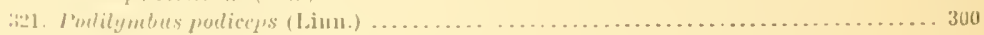

MLCIII

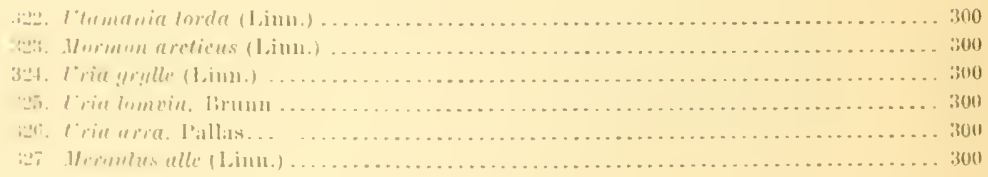

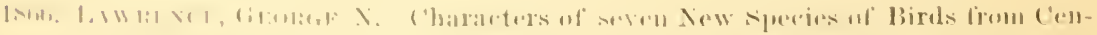

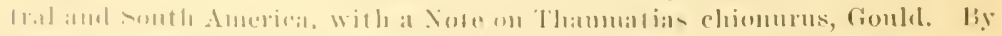

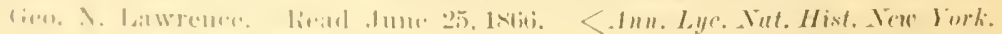

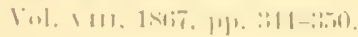


18ibi, L.A

5. J'herthormis ressinit 11 .

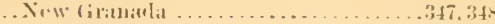

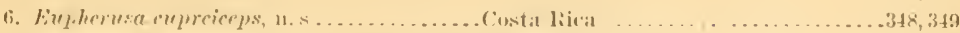

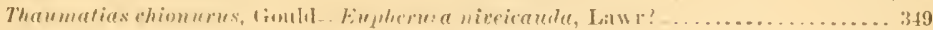

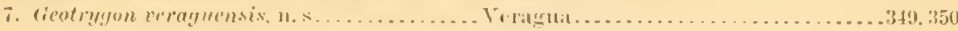

48.

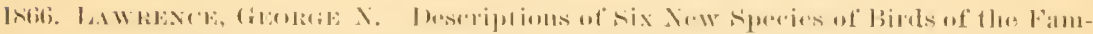

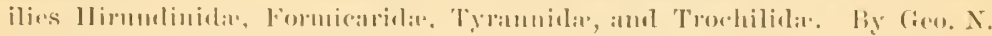

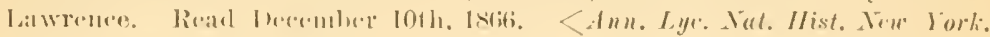

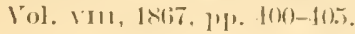

HIIIIIINII).K.

1'age.

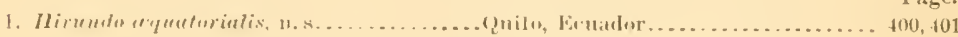

FUI:MIIAIII.

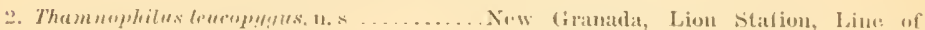

l'anami Railruad ...............411, 102

TYRAXXII.F.

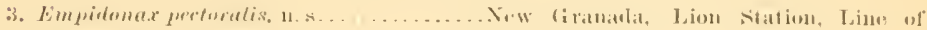

I':tnamit liailıoad................. 102

JHUCHILIH.E.

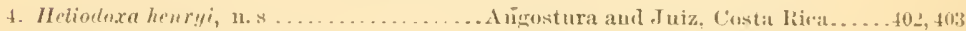

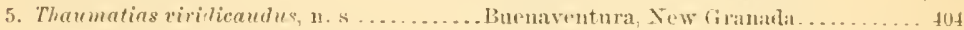

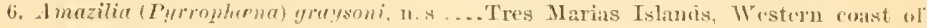

Mexire..................... f(1)4, 4105

49.

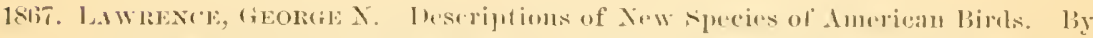
fieo. X. Lawrence, liead May 13, 186it. Limn. Lye. Lal. Hist. For Jork. Vol. vili, 18it, Pi. Atif-482.

1.

1. Fuphoniu purpurea. n.s..............

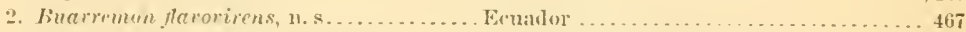

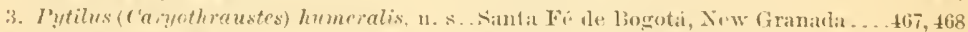

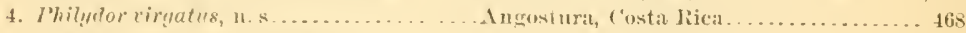

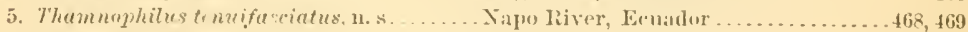

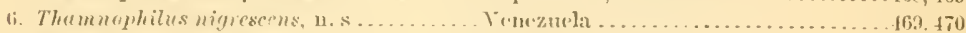

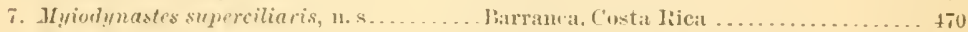

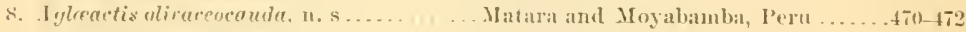

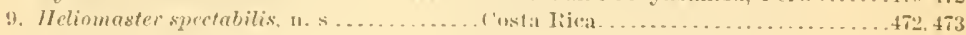

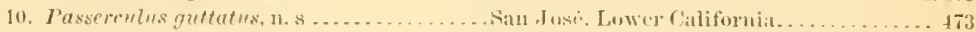

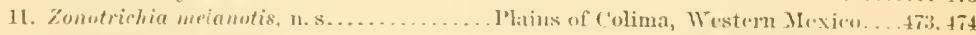

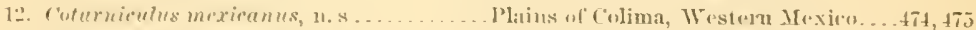

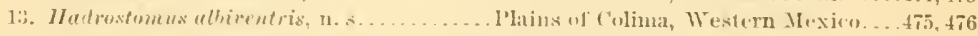

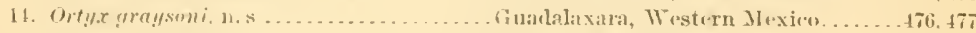

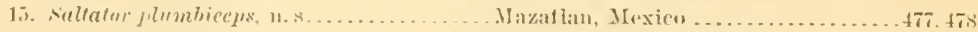

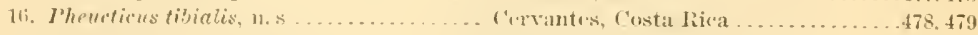

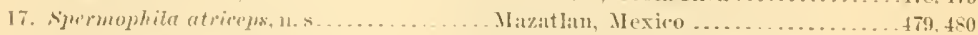

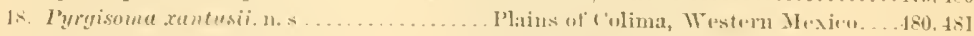

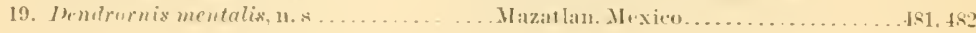

50.

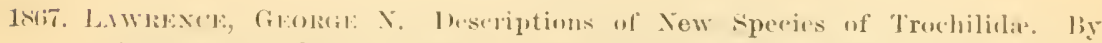

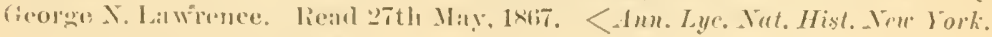

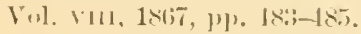

1. Merichu bryanter, n., s.

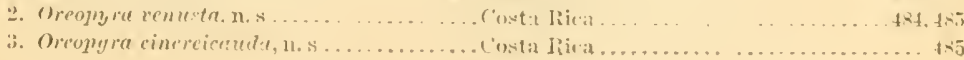


51.

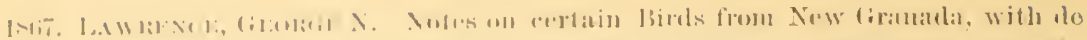

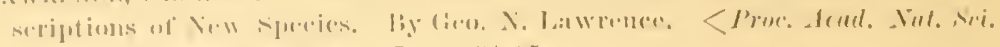

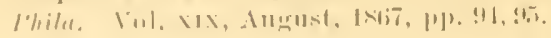

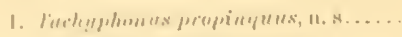

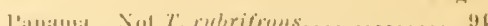

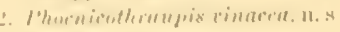

New (i raumla, Line of Jamimat liailruat. 94

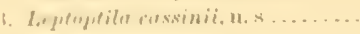

52.

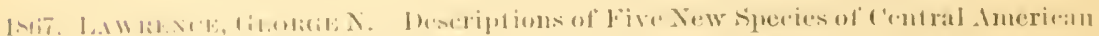

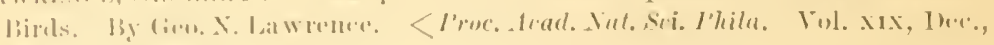

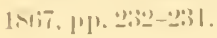

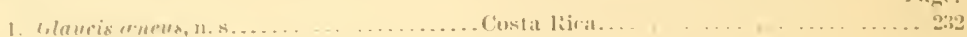

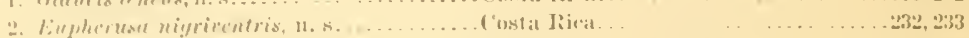

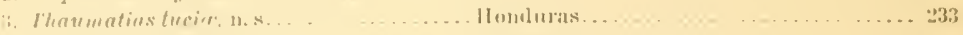

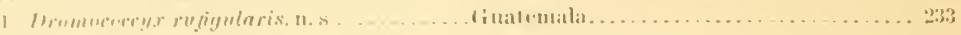

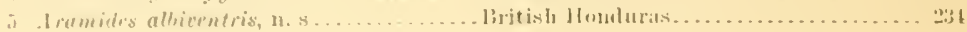

53.

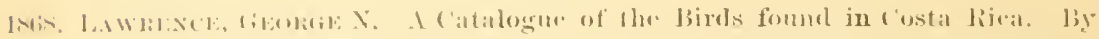

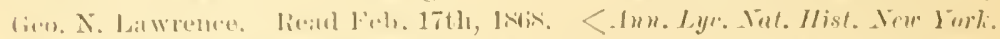

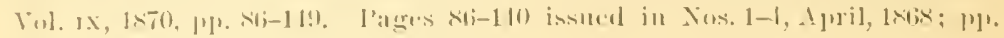

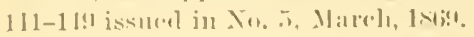

'T['RI)II, lis.

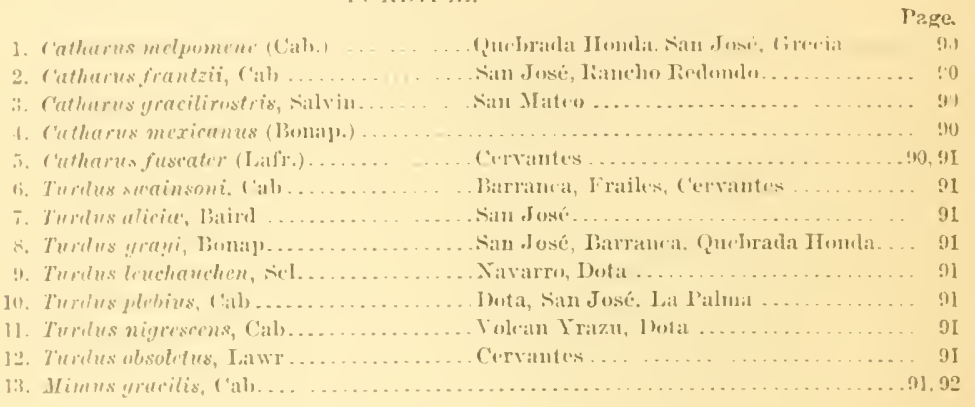

('IXI'LIIS.L.

11. Cinches urdesimens, Nils.

Intit......

SII.11111.1:

15. Iolioplitu sufwriliaris, Latwr.

Anerstuma, Miro, linimil

92

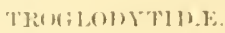

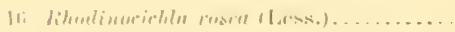

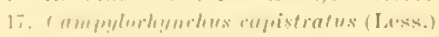

18. I'mmunlorleynehes zunatus, Jass.

12). I"Iplustiuns lewrostictus, ('al)

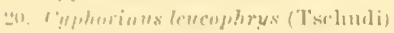

¿1 ruphurinus lemerencei, Sell

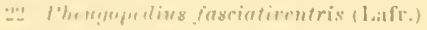

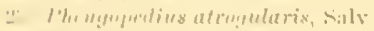

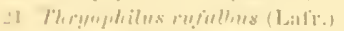

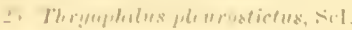

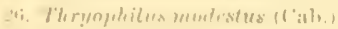

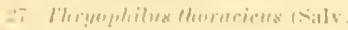

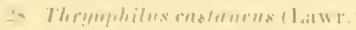

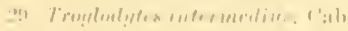

dil. Trmoloulyter unymictus, liairil

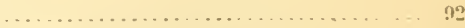

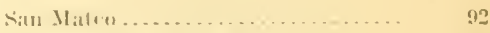

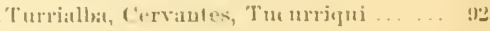

Aumostura, Turriallna .............. 92

siul Jusé ....................

Allgostura . . . . . . . . . . . . . . . . .

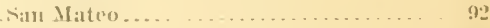

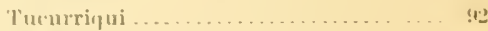

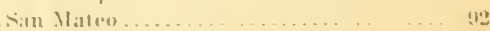

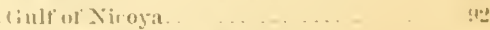

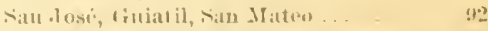

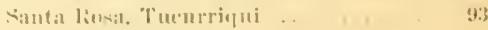

l'alliure, dugnstura ...... ....... 93

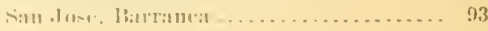




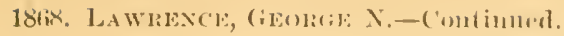

SYLYITHAII.T.

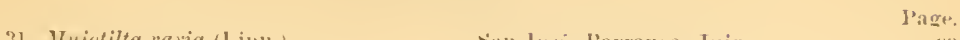

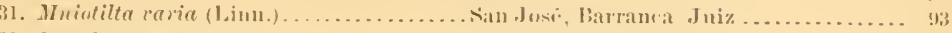

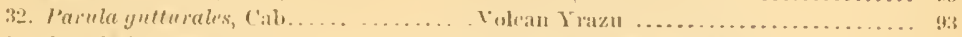

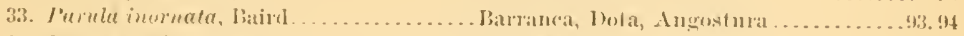

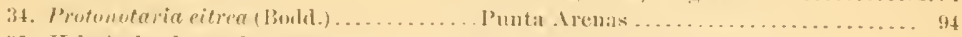

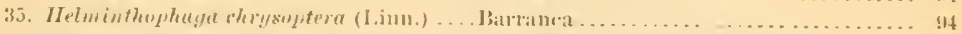

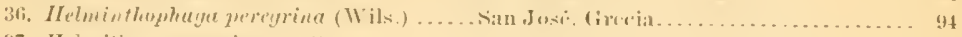

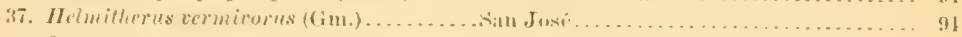

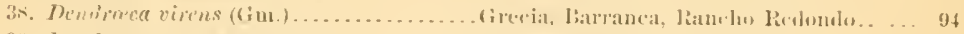

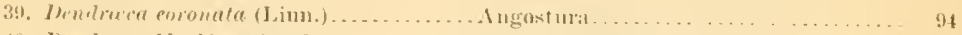

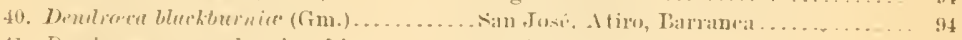

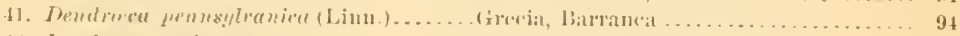

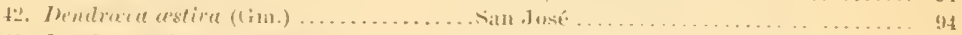

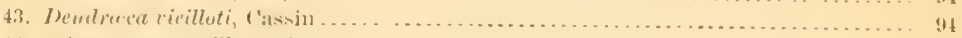

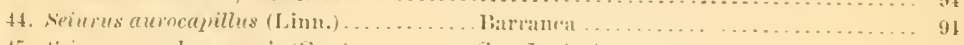

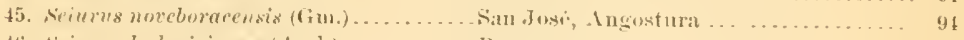

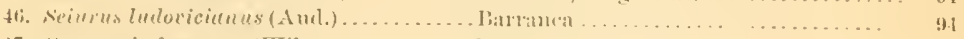

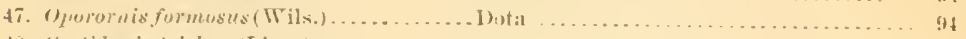

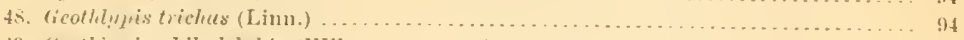

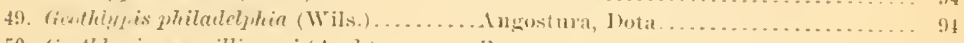

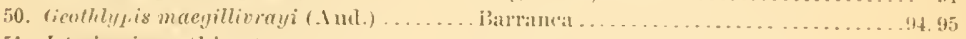

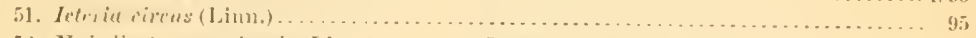

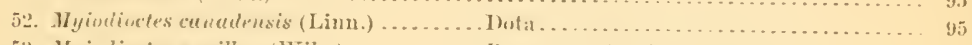

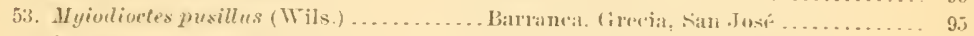

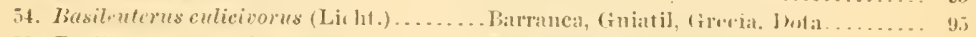

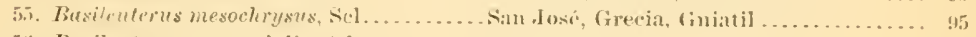

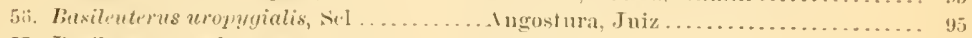

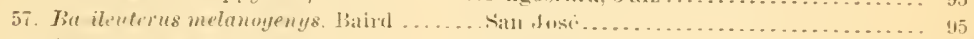

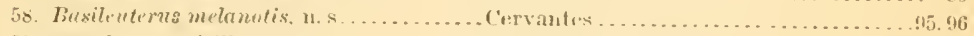

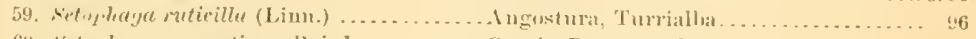

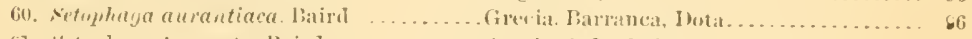

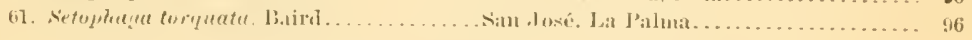

HIRT XIHXTDE.

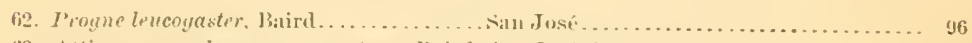

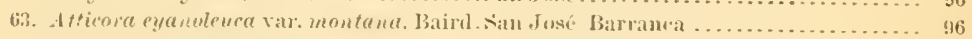

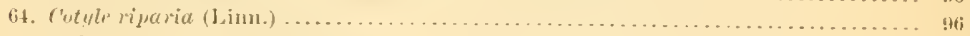

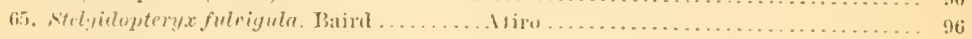

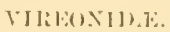

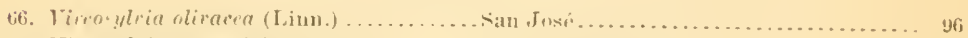

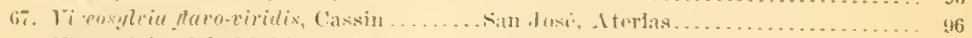

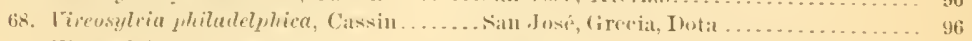

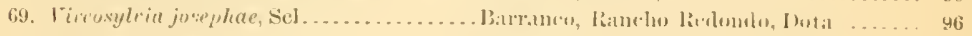

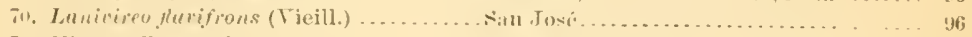

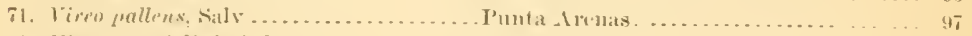

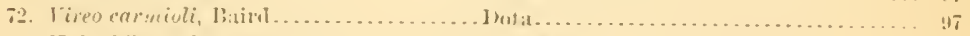

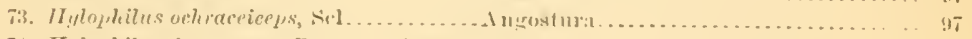

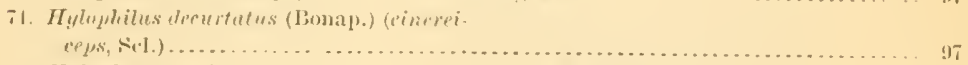

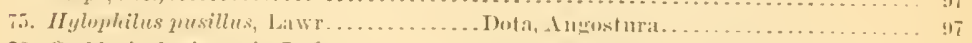

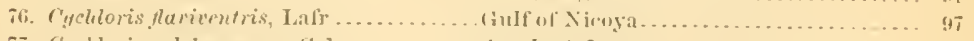

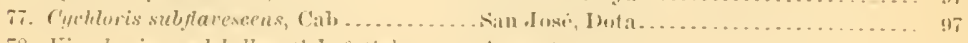

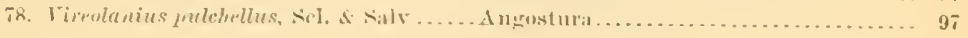

IMIPLIII.E.

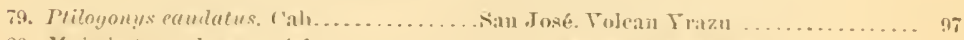

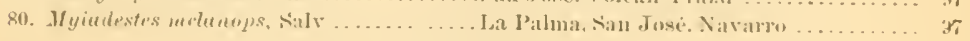

IELELIIt. T\%.

81. Miglossa plumber, ('ab

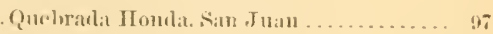

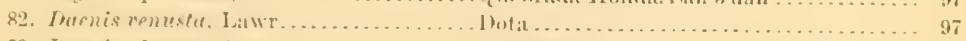

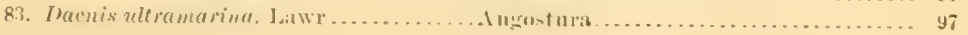




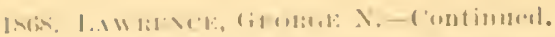

Page

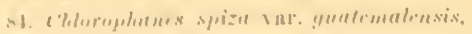

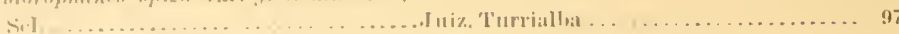

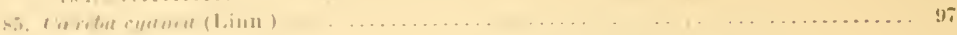

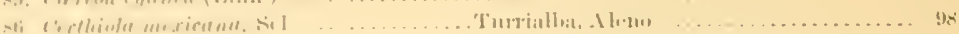

T.INilikils. I:

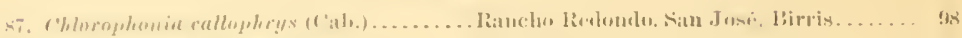

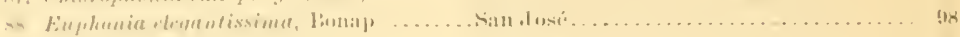

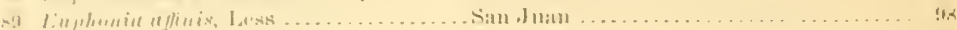

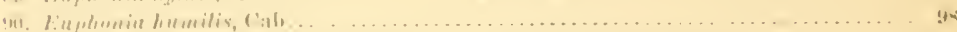

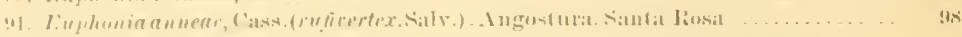

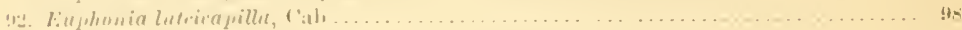

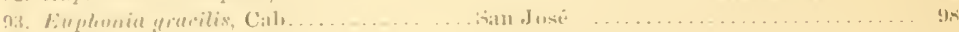

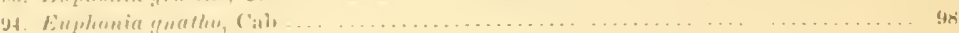

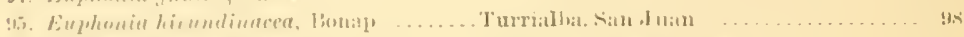

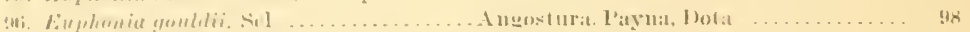

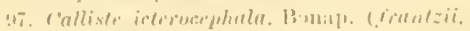

('ish.)

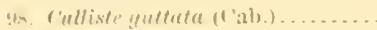

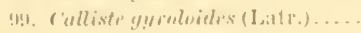

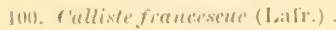

111. C'alliste durvii, sills......

laz. Fana we diatemus (Lass.)

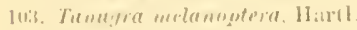

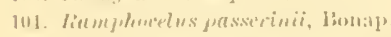

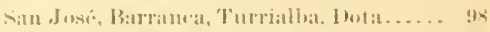

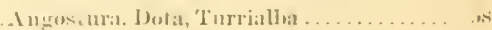

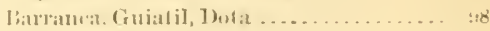

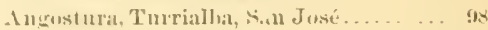

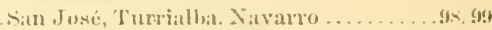

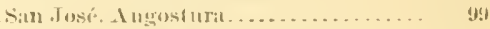

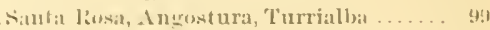

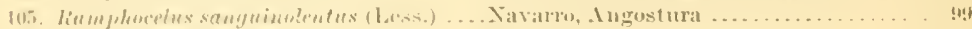

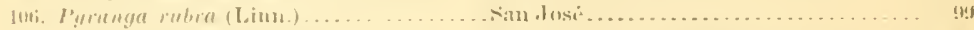

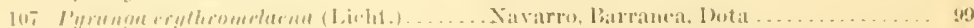

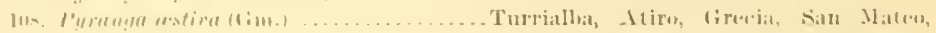

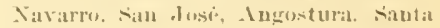

Jiosa....................

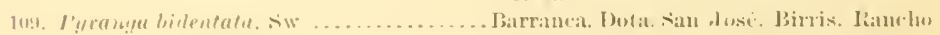

lievirmulo ...................... 99

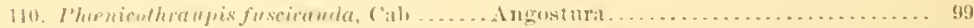

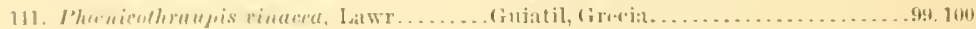

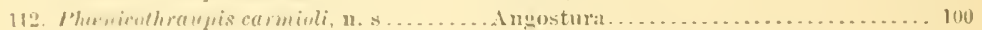

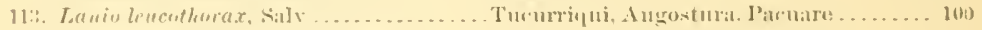

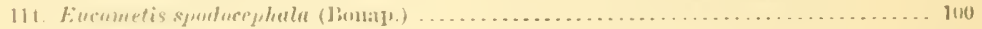

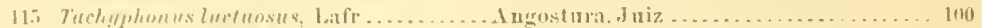

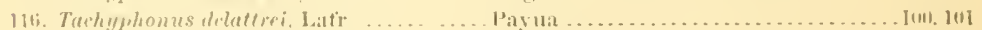

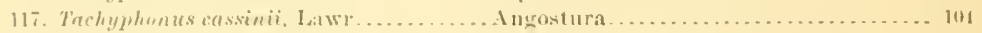

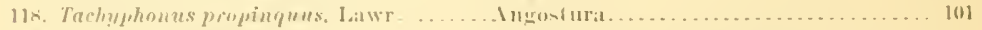

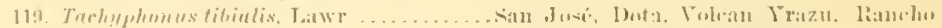

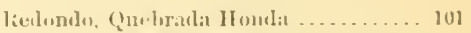

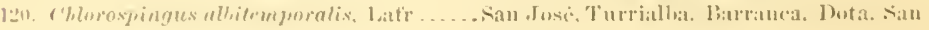

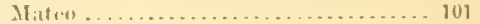

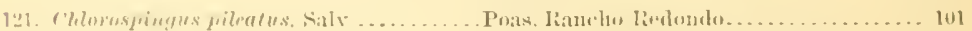

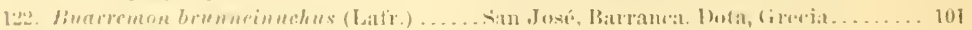

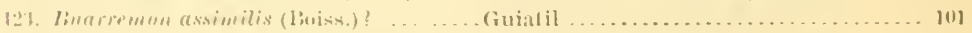

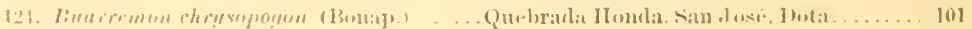

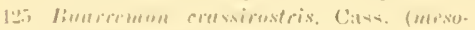

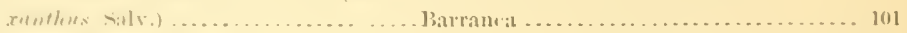

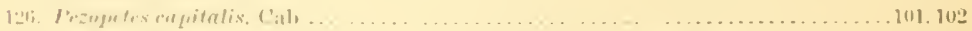

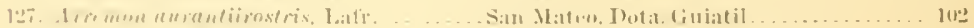

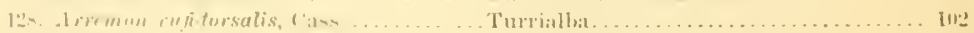

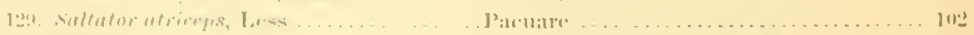

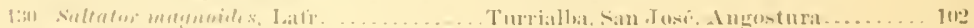

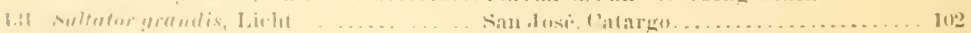

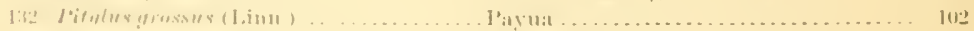

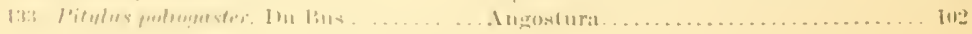

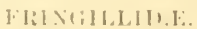

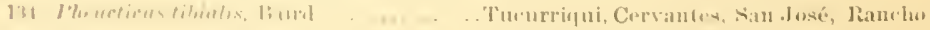

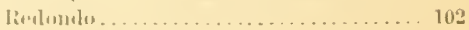

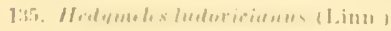

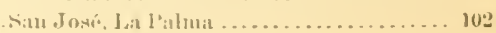




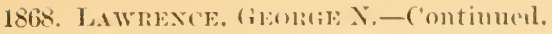

l'age.

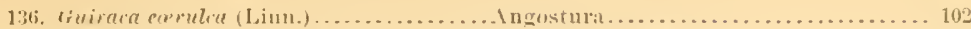

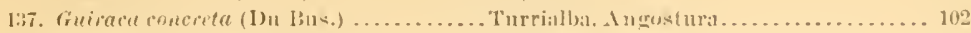

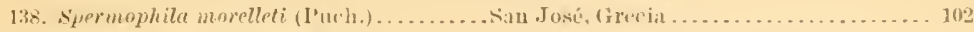

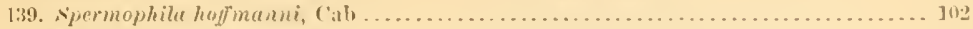

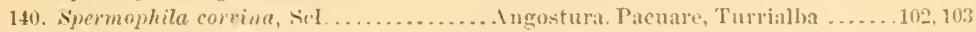

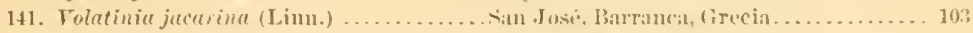

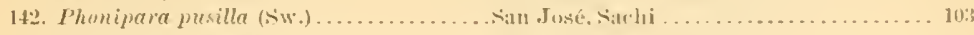

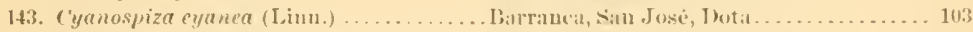

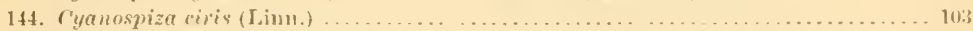

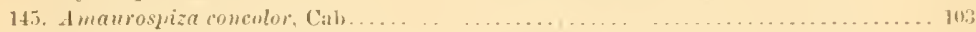

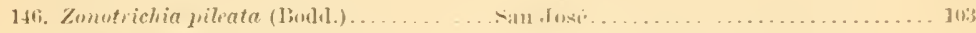

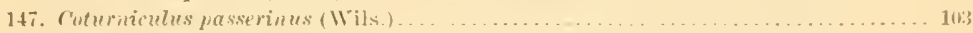

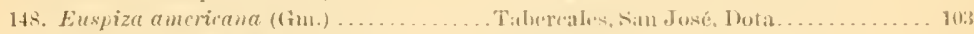

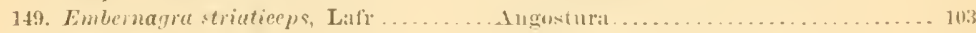

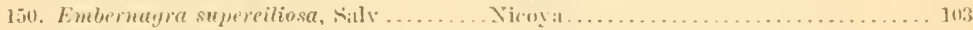

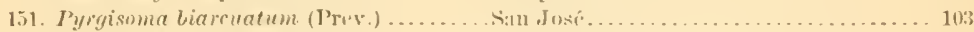

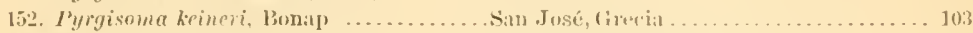

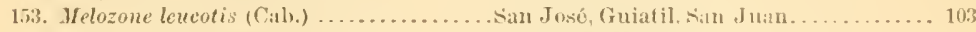

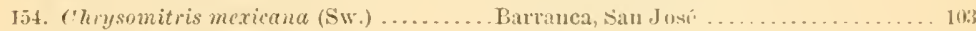

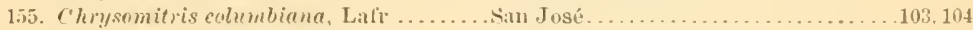

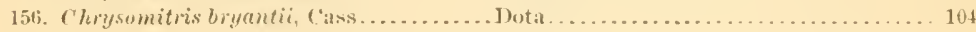

16"TELI11.E.

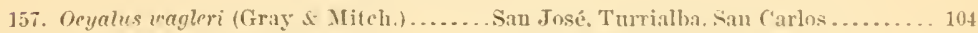

158. Ostinops montezumac (Less.). .......... San Carlos, Angostmat.............. 104

159. Amblycercus prevosti (Less.) . . . . . . . . . san José. Turrialba . . . . . . . . . . . . 10. 10.

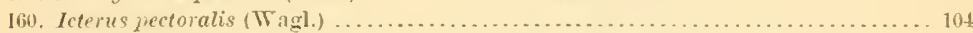

161. Jeterus salvini, Cass................ Turrialla. ...n Carlos . . . . . . . . . 104

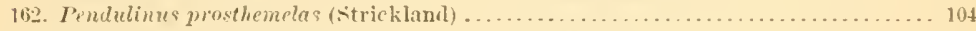

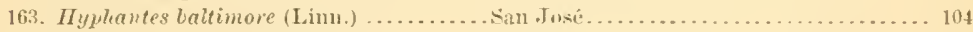

164. Santhorntes s.urius (Linu.) . . . . . . . . . San . José . . . . . . . . . . . . . . . . . 104

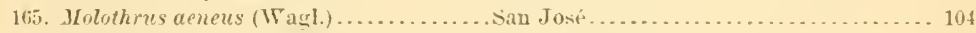

16if. Ayelaius phoniceus (linn.) ...........Gull of Nicoya.................. 104

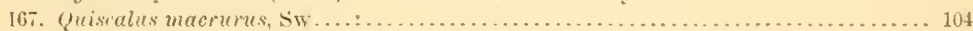

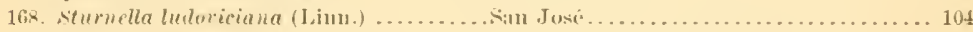

(ORTII).E.

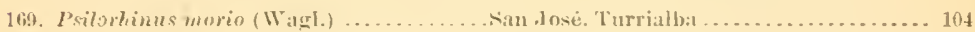

\section{HE.TIR() ')L.A ['T1D.E.}

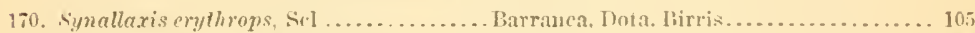

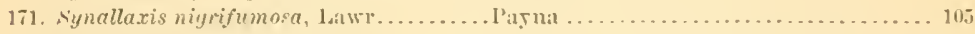

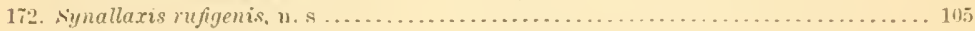

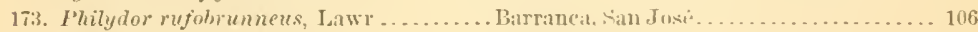

174. Philydor virgatus, Lanr............ Angosturil................... 106

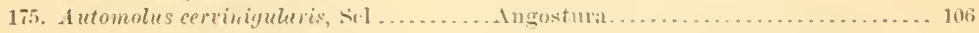

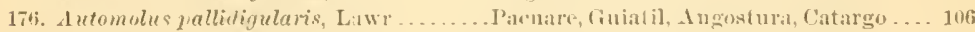

177. I utomolus rufescens, law ........... Rirris........................ 106

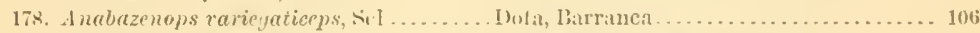

179. Anabaznops lineatus, Lawr.......... Ingnstura, Birris, C'Prantes .......... 106

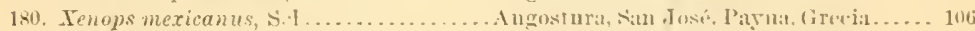

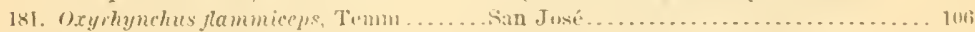

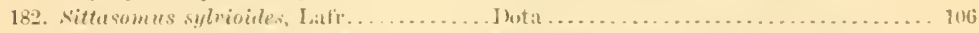

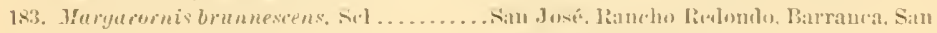

Mator, Birris ..................... $100 ;$

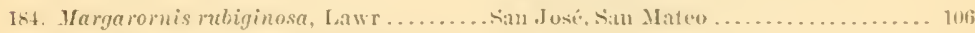

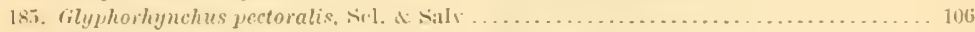

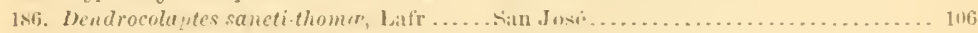

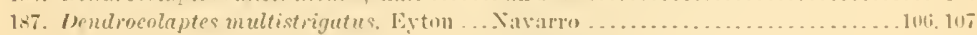

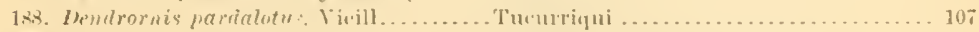

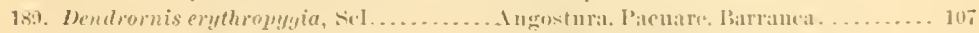

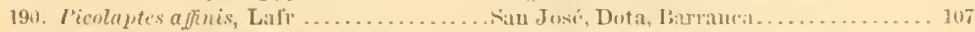

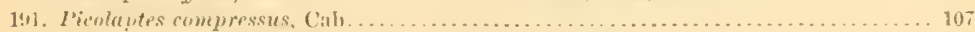

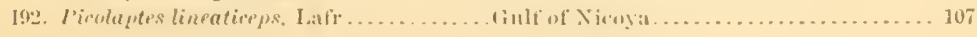




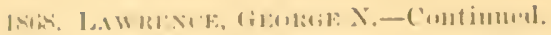

[F(1):J](:A]:I1) R.

I':ast:

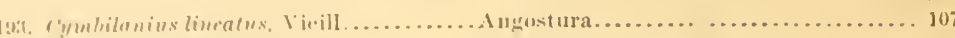

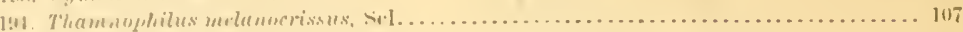

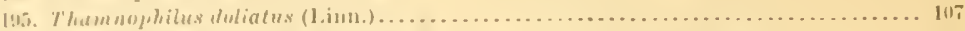

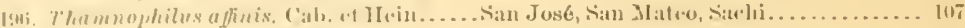

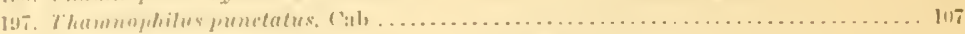

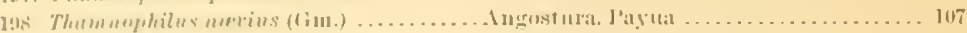

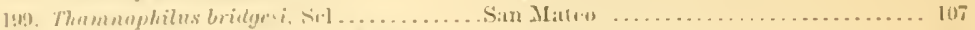

2nu. Thum

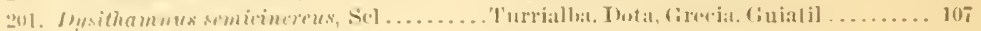

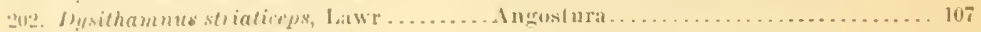

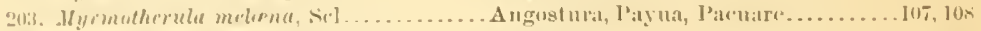

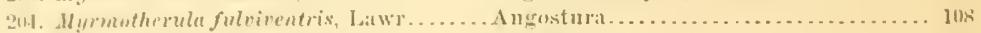

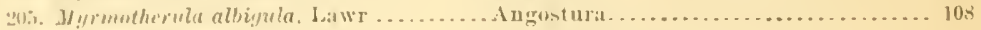

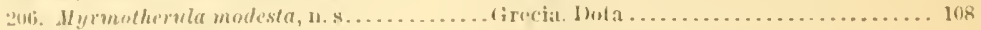

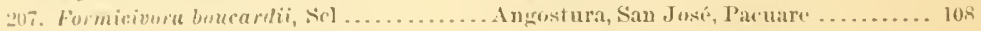

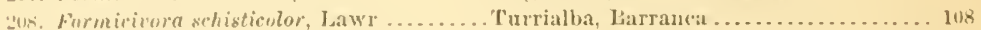

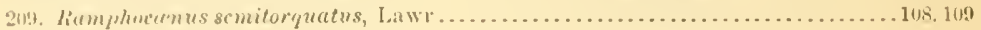

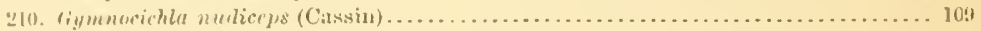

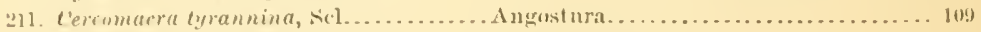

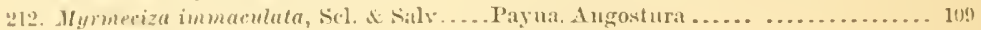

213. Myrmeriza lamosticta, Silv .......... Tu.urriqui .................... 109

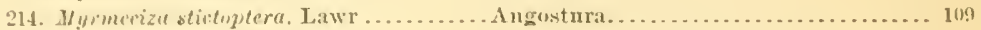

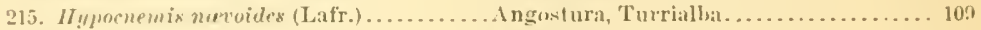

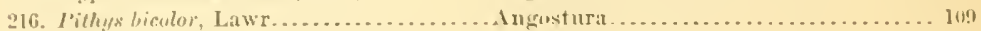

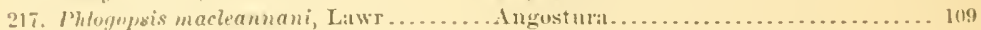

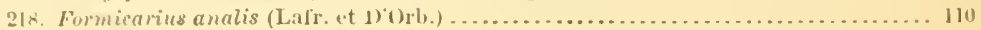

219. Formicarius holfmanni, (ab..................................... 110

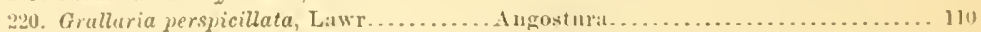

221. Girallaria diecs, Salv ............... Tucurriøui ................... 110

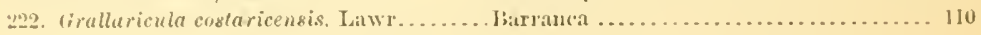

\section{TYIANNIIIE}

223. dtila selateri. Lawr.............. Guiatil ...................... 110

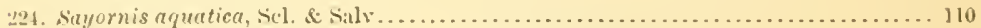

2.5. Copume leveonotus, Lalir .................11 Jusc, l'acuare................. 110

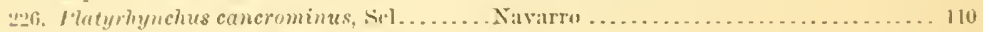

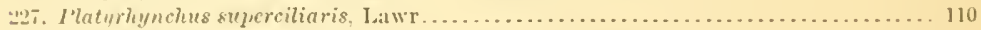

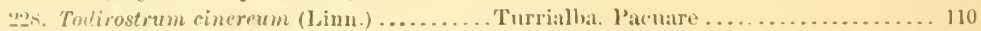

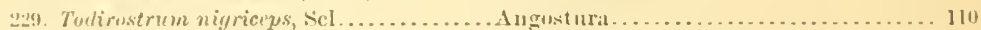

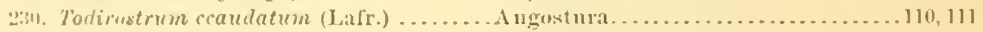

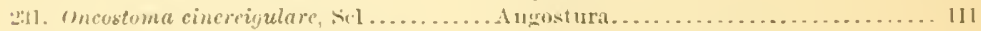

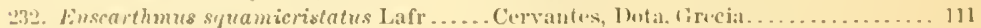

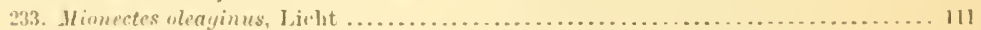

234. Mionectes assimilis, ..ं. ............. Angustura. (iniatil, Payna .......... 111

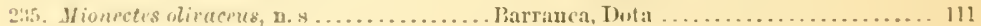

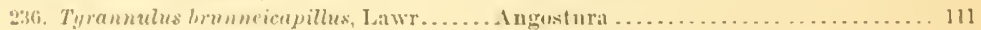

237. Tyranniscus villissimus, ticl. \& sillv.... Ingostura, Doto, Turriallya, liarranea... 111

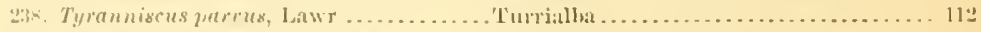

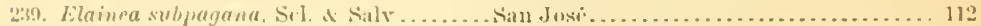

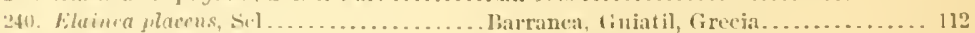

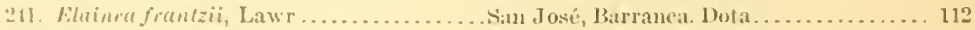

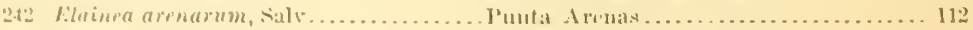

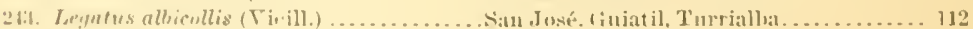

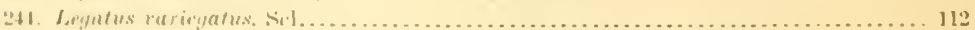

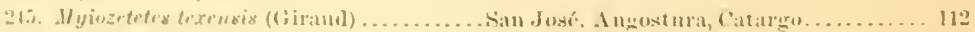

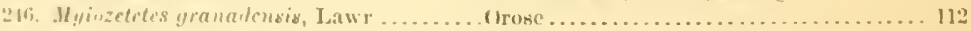

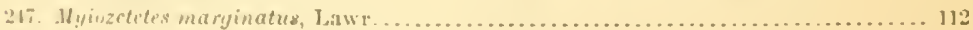

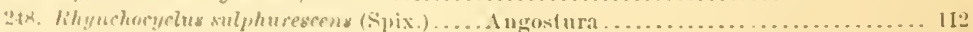

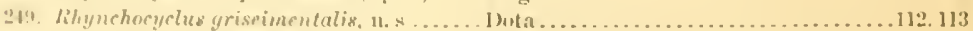

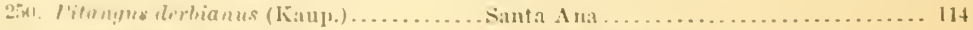

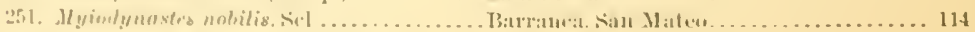

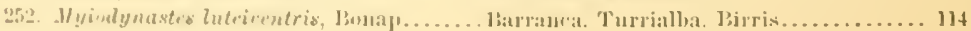




\section{PUBLISHED WRITINGS OF GEORGE NEWBOLD LAWRENCE. 41}

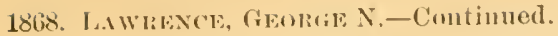

1'uge,

253. Myiolymustes hemichrysus, Cah. (super.

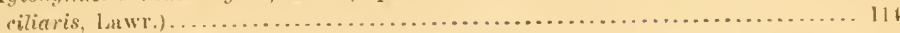

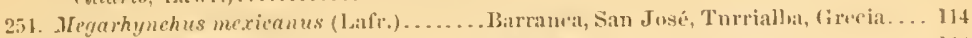

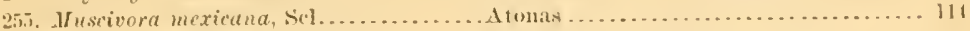

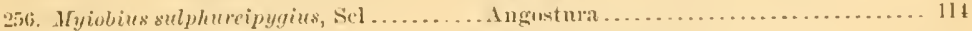

257. Myiobius erythrume. ('ib ............ Angostura, Patuar' .............. 111

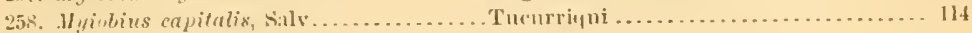

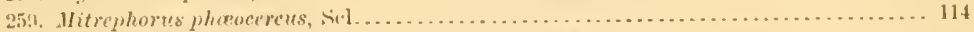

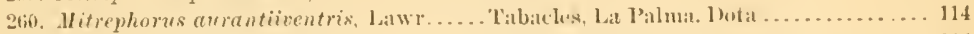

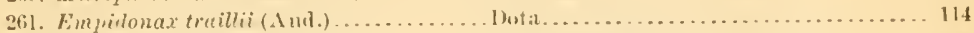

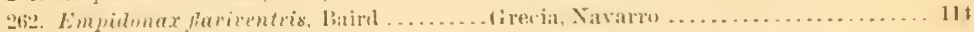

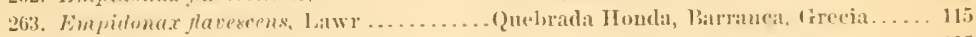

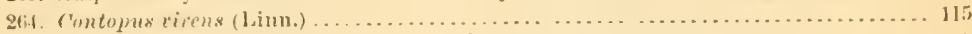

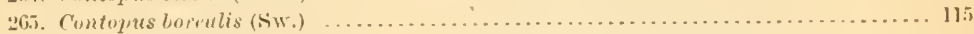

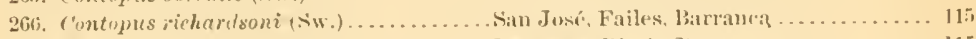

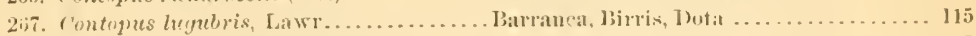

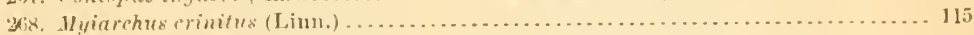

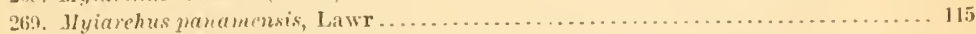

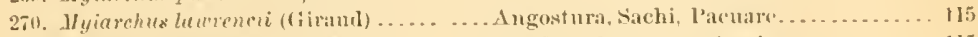

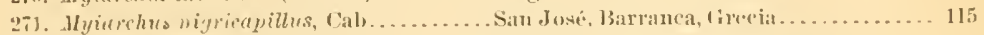

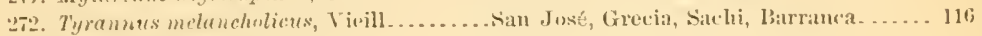

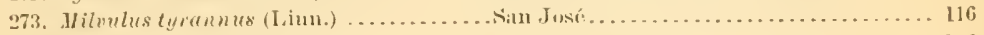

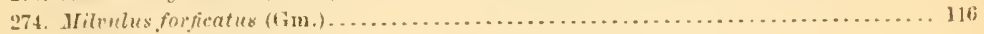

\section{COTINGID.E.}

275. Tityra pessonata, Jarl. \& Selby . . . . . . San Jusi, Guiatil, larranea ............ 116

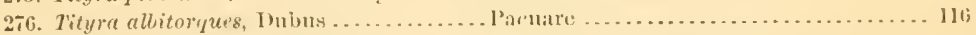

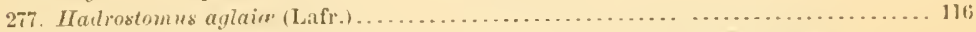

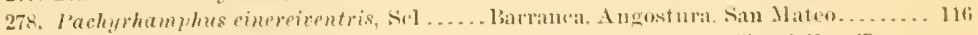

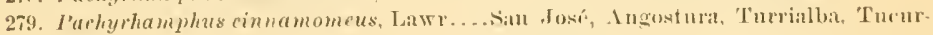

rinini.......................... 116

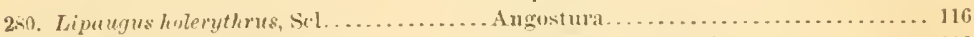

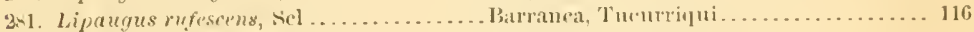

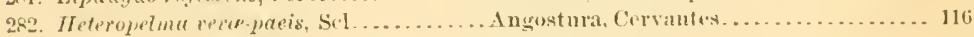

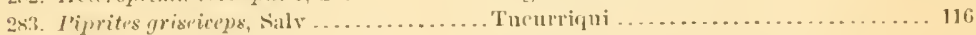

2st. Jipre mentalis, siel ................ Angustura, Paiz, Tueurriyui.......... 11f

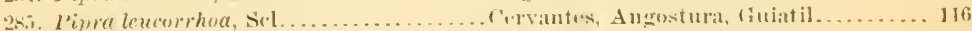

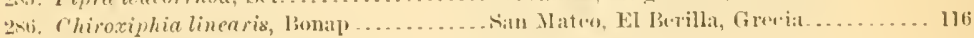

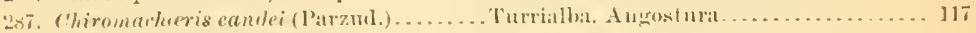

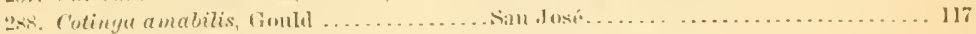

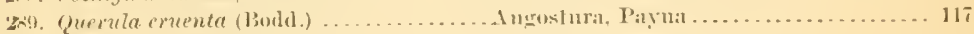

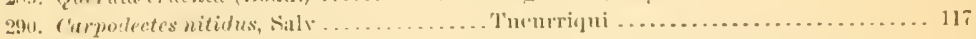

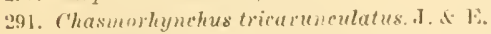

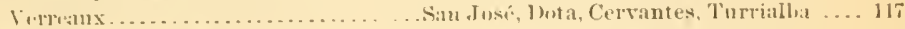

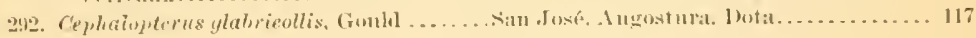

\section{MOMOTI1) E}

293. Yomotis mavtii, spix .............. I'atuare ....................... 117

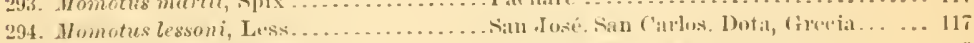

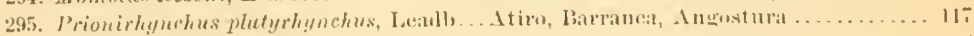

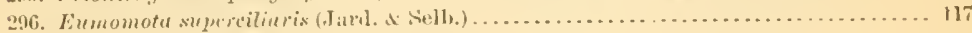

\section{ALCWLIXIDA}

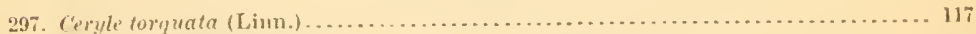

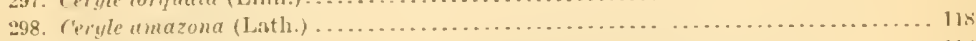

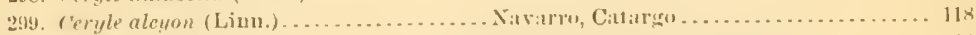

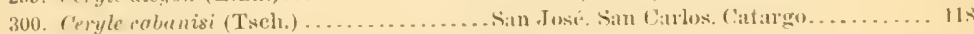

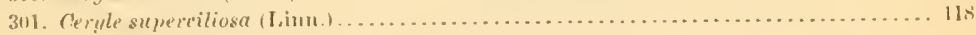

G.ALIBITINA.

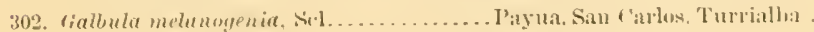




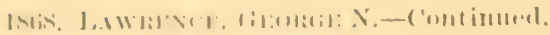

$131 \% 1 \%, 11 \% . \mathrm{F}$.

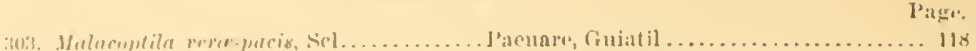

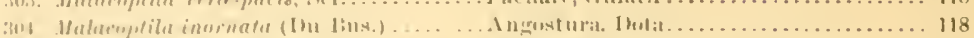

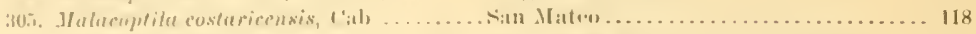

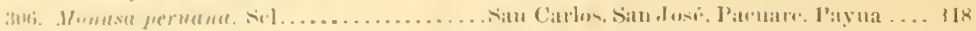

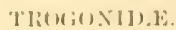

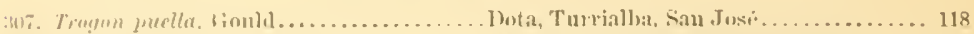

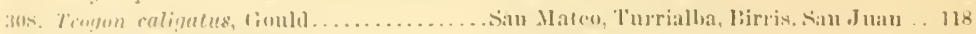

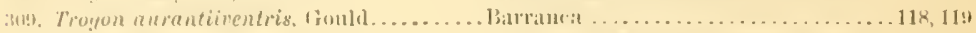

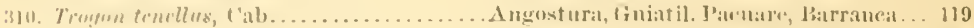

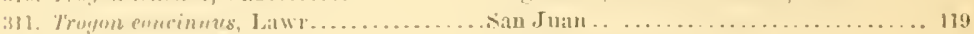

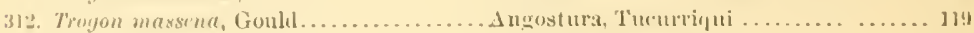

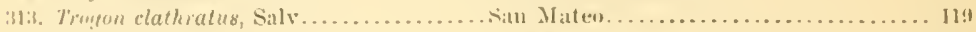

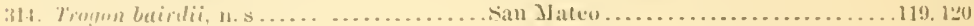

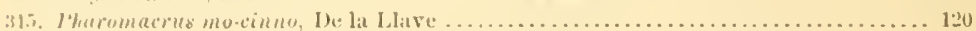

1.APLIMLLGIH,E.

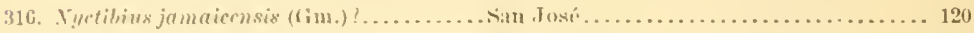

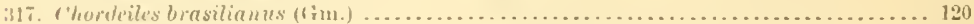

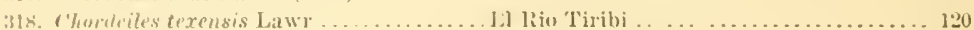

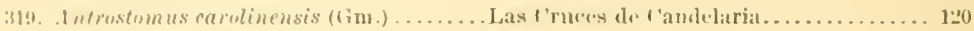

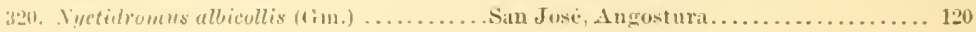

T1:MIHLIH.W.

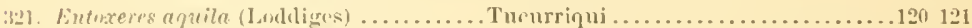

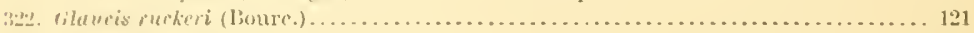

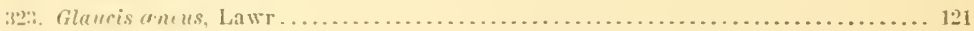

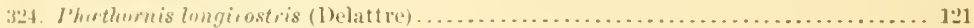

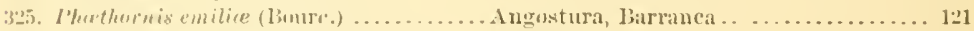

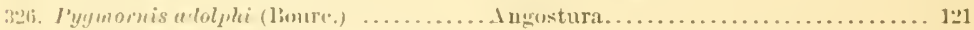

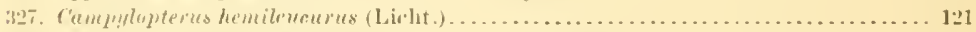

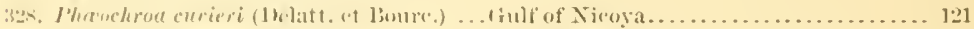

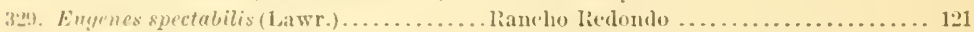

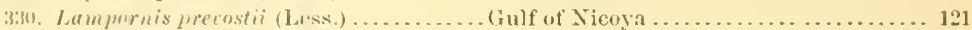

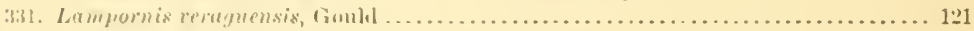

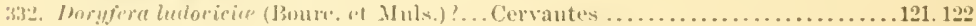

33is. ('balybura melenmrhou, Nalv. (C. cami-

wi. l.nwr.) ..................... Angosturn, Pacuare............. 122

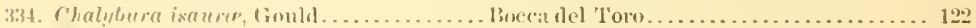

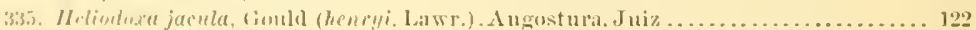

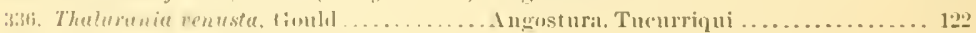

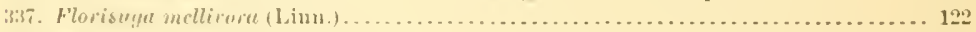

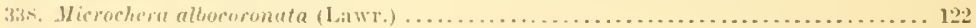

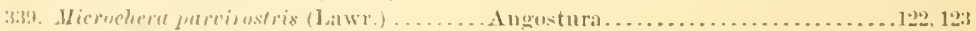

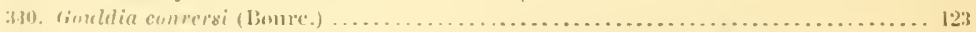

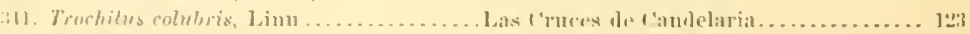

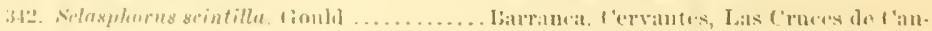

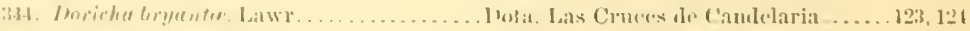

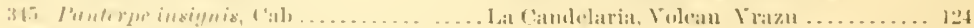

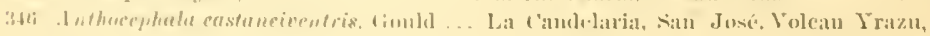

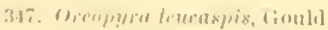

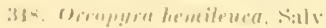

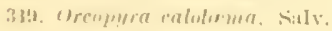

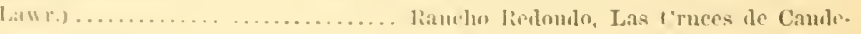

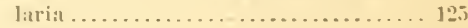

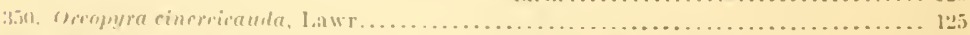

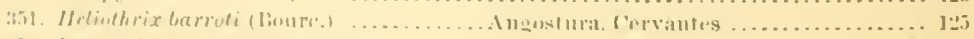

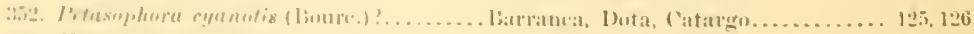

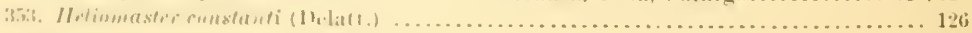

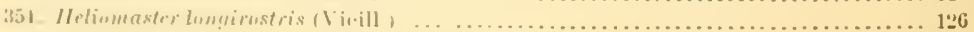




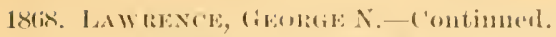

$1:: 1: 0 \%$

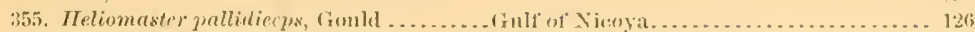

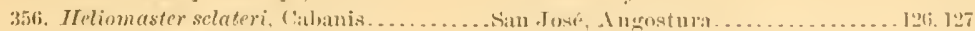

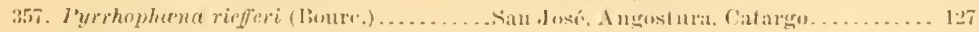

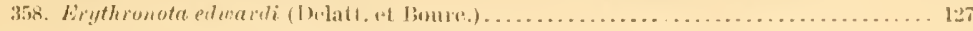

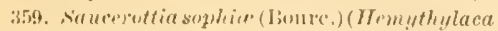

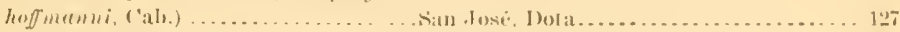

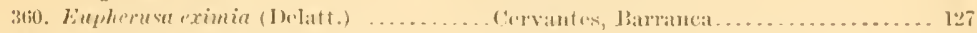

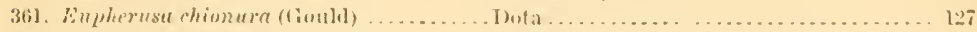

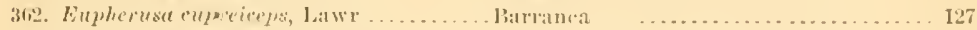

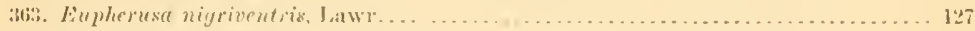

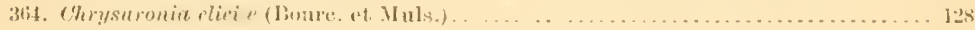

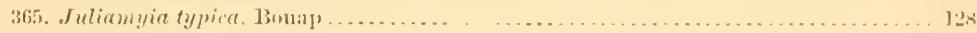

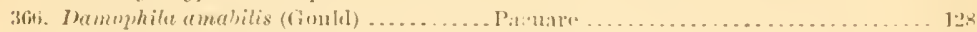

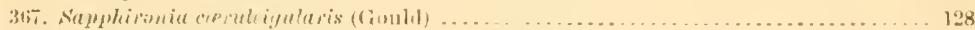

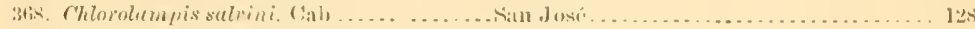

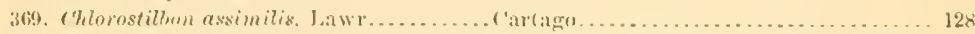

\section{('I'ILIJ)E.}

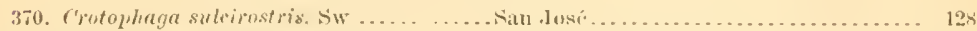

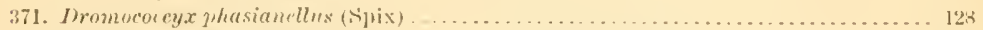

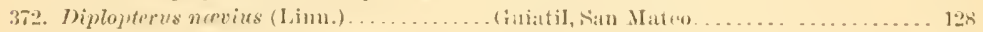

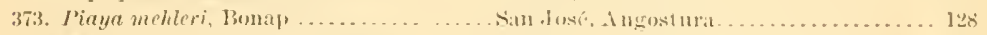

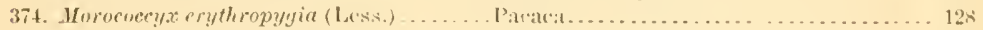

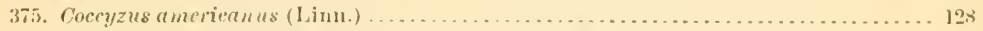

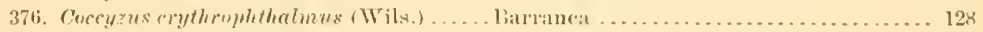

\section{RAMPHASTIIUE}

37. Ramphastes tocart, Virill............. Ingustur'a, San Carlos, Turrialla ...... 128

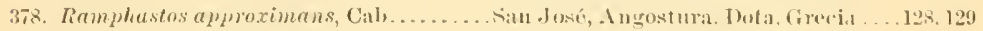

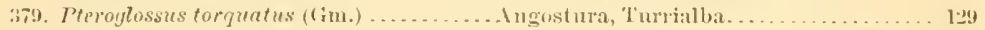

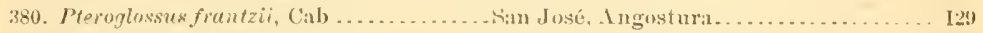

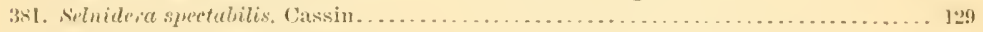

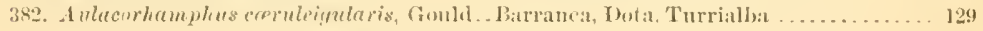

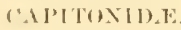

383. Crpito bonveieri (Latr.) 3it. raprito hartlambi (Latr.) 3*5. Tetragomes frantzii. Ni.I
Marranc: Turrialja. ................ 130

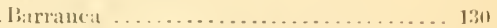

Sim Jusi, Cerviuldes, Navarro, Birris, la

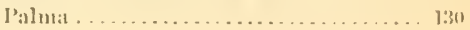

I'IIII.F.

386. Campephilus guatemalensix (Hart1.) . . . . san .Iust, Angostura, fireria ......... 130

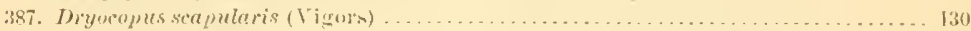

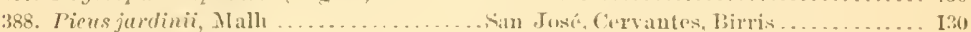

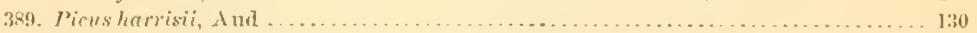

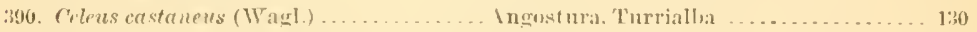

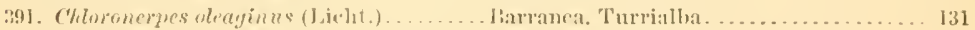

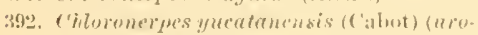
puginlis, (als.) ................ Thurriallat, Barrauca............. 131

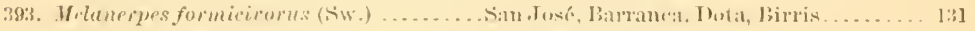

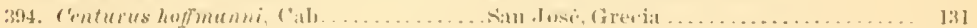

395. ('enturus yerini (Trmms.) (puelereni,

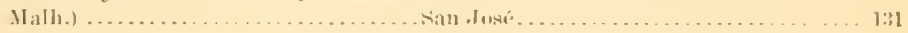

I'SITYICHI.E.

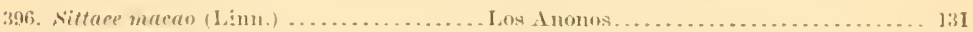

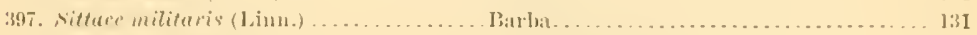

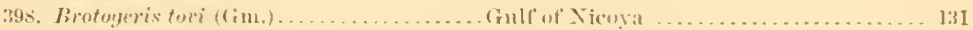

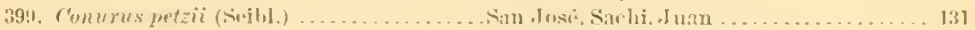

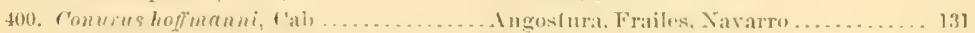

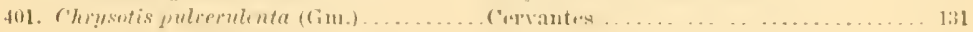

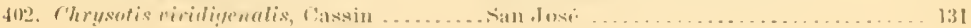




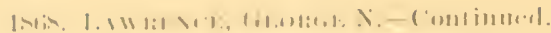

1'a:re.

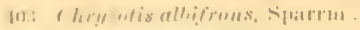

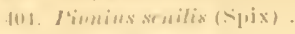

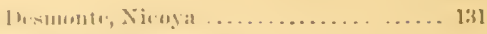

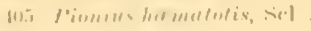

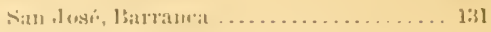

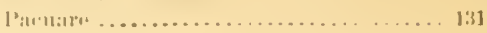

S'll:It11)

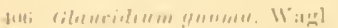

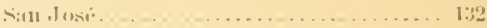

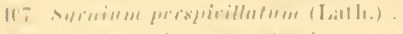

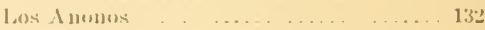

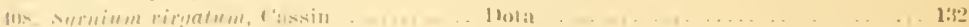

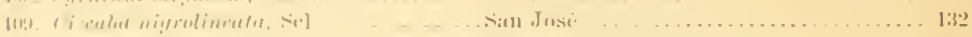

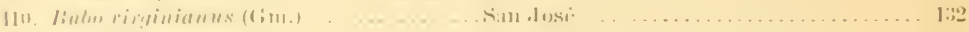

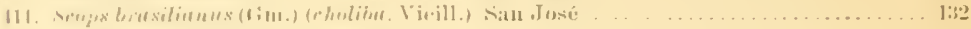

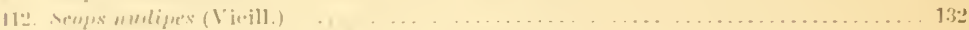

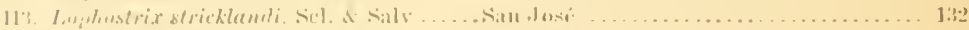

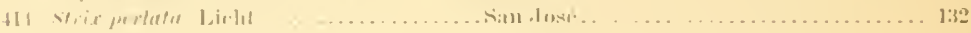

I.AT (1) II).E.

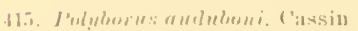

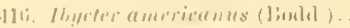

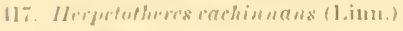

II. sprizatus armatus (1)amil.).

41!. spizurtus tyiamuแs (Max.)

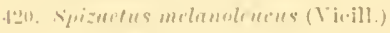

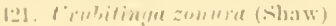

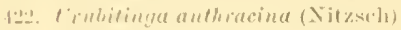

I2:3 Jinter horealis var. montenus, Xutt

121. Sintro jem sulvanirus (IVils.) ........

1:-5. Sintere erythromotus (King)

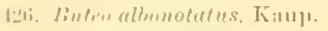

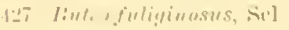

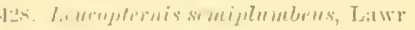

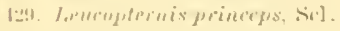

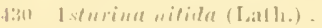

l:31. Istmina ma!mirm.tris (Gin.).

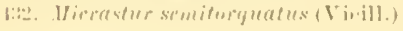

1:3. freprifer fuscus (lim.) .

1:4. Lecipiter piluates (Mlax.)

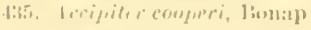

[:66. Jinnunsulus sparretus (].inn.)

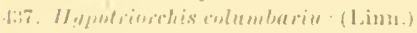

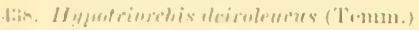

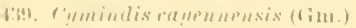

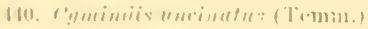

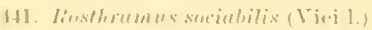

1f2. Rimusiles furratus (1 ir.jll.)

Ha. I imes helsenius (1.jun.)

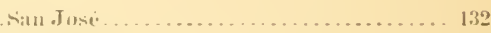

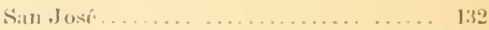

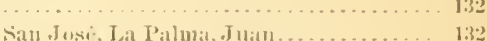

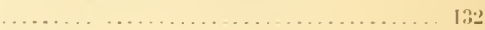

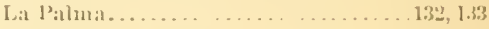

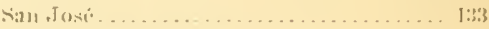

S:ıll . Tosé, A newat urat . . . . . . . . . . . . . . 133

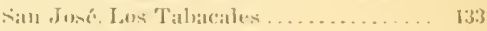

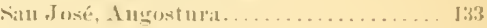

Sitn Josi, titu Intnnio. . . . . . . . . . . 133

Sin Juxé.................... 1:33

Lit l'alma . . . . . . . . . . . . . . . . $1: 33$

$\ldots \ldots \ldots \ldots \ldots \ldots \ldots \ldots \ldots \ldots \ldots \ldots \ldots \ldots \ldots$

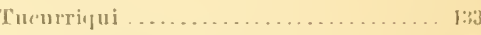

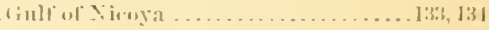

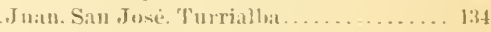

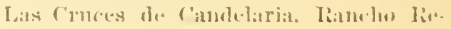

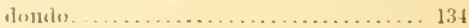

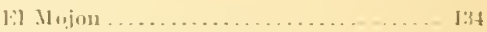

Sils Juxí, llutat. Turvialloal . . . . . . . . . I:H

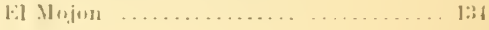

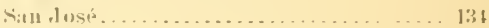

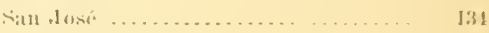

1,i P:1lntat ... . . . . . . . . . . . . . . . . 1:31

fiulf $u f^{\circ}$ Nie.oya ..................... 134

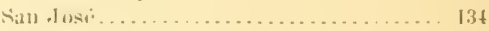

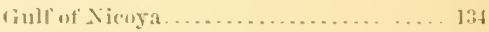

l'irris. . . . . . . . . . . . . . . . . . . 1:4

nill buat ........................ 134

\section{IITITIII.T:}

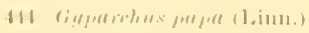

tinll ut Ximy:

$1: 3$

$(11,7 \times 11 \% 10.1 \%$

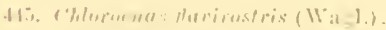

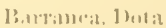

134.1 .35

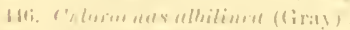

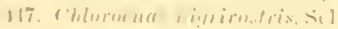

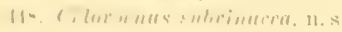

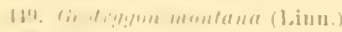

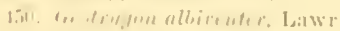

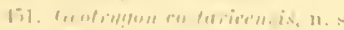

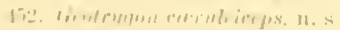

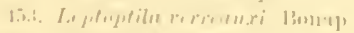

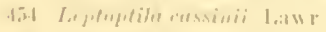

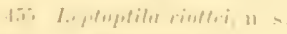

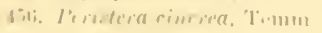

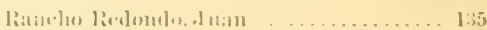

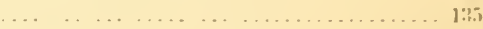

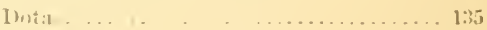

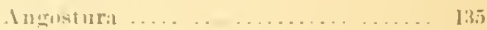

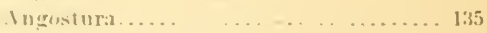

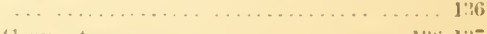

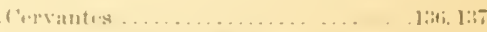

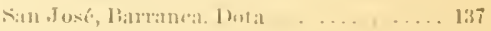

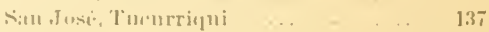

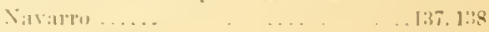

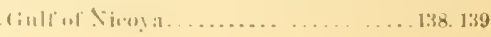




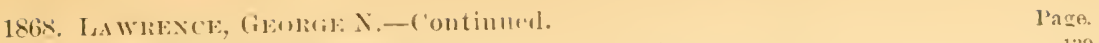

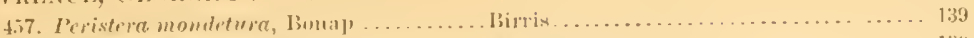

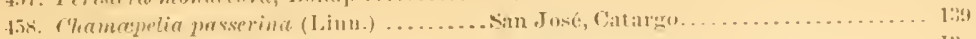

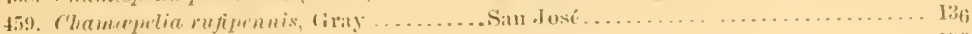

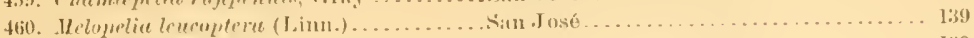

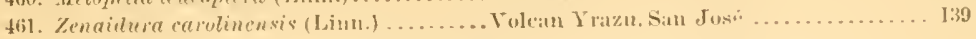

\section{PENEIAIPIIE.}

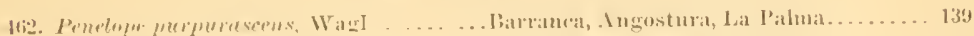

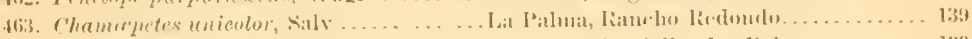

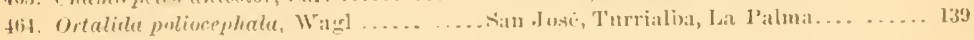

('RACIII)

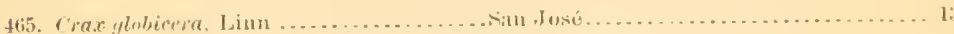

\section{I'ERIII'II).E.}

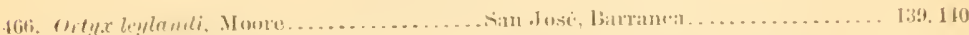

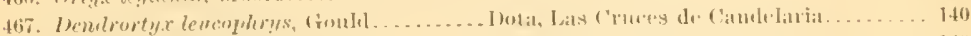

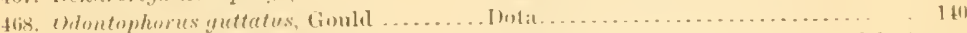

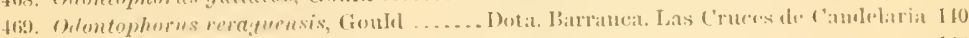

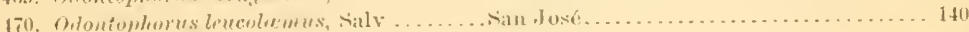

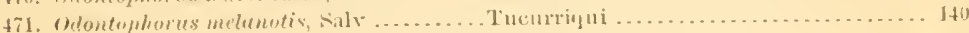

\section{('IVYTUTIITA.}

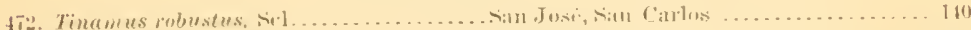

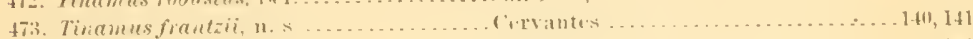

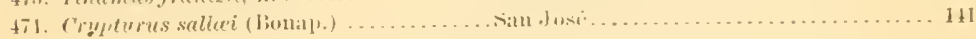

('H.IIIIIIII1)E.

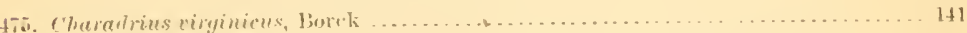

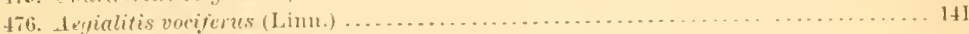

\section{IEITT()IPI)II). F.}

47. Huematopus palliatus, Titmu.

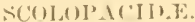

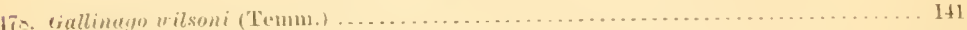

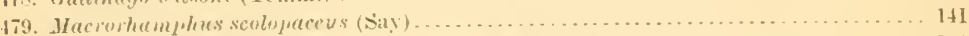

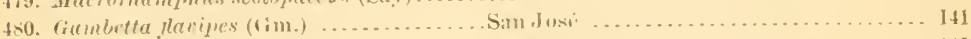

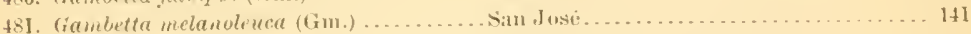

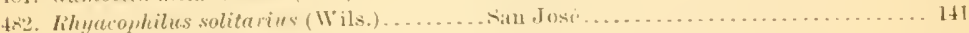

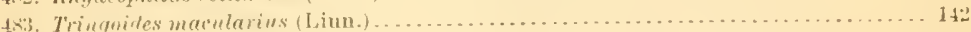

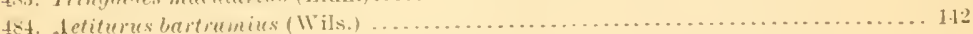

TANTALII.1\%.

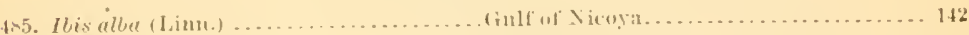

IPATALEII)E.

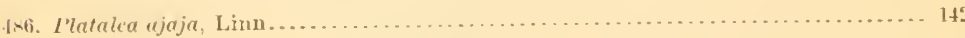

('ANCIRAIII).

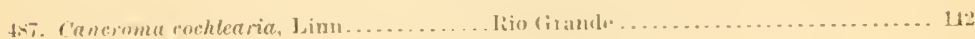

\section{MRIIIIS.}

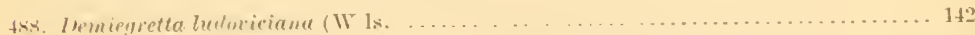

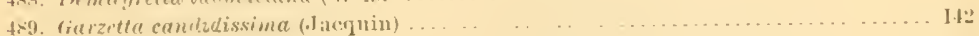

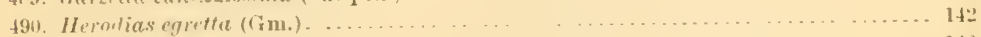

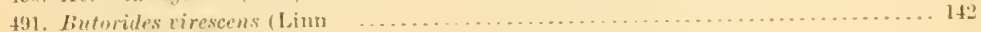

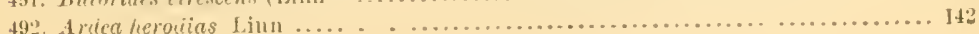




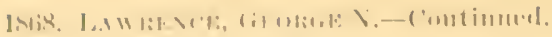

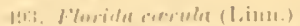

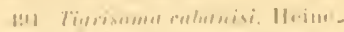

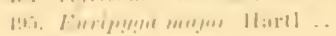

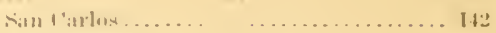

1112uturi......................... IH2

Ii.II,I,III.K.

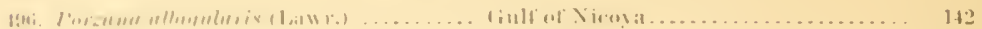

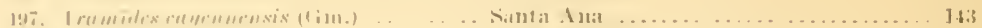

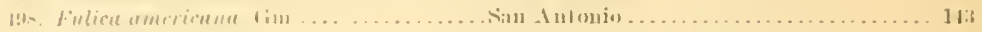

1. . . 1 1110.1\%.

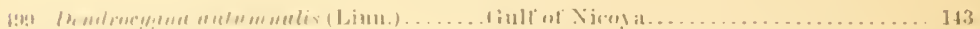

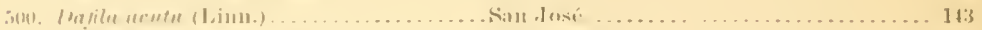

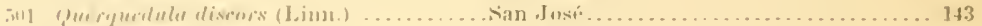

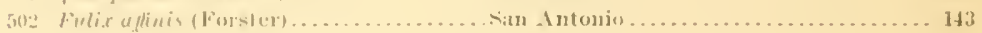

PIATII.T:

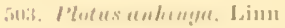

liult ol Nirovit.

I'UIICIIII.F.

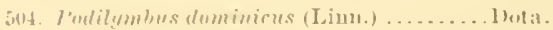

Al'will.

TYRAXXII).K.

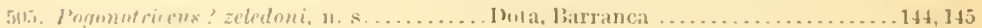

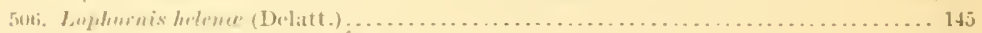

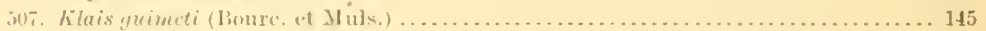

PSITHCID.E.

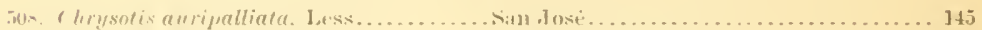

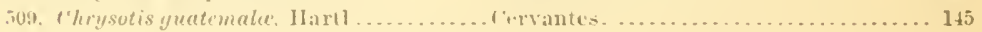

F.LCONID.E.

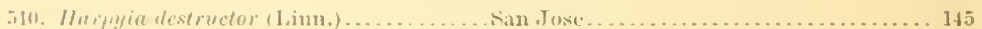

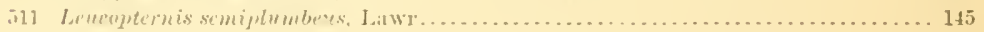

\section{NiTr:}

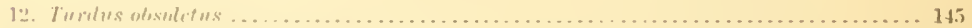

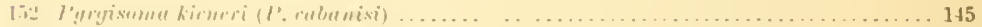

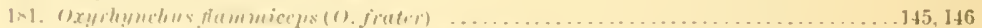

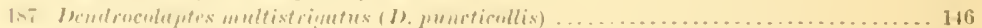

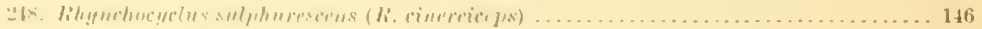

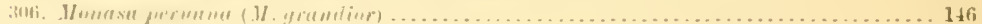

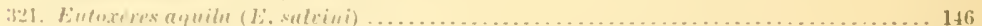

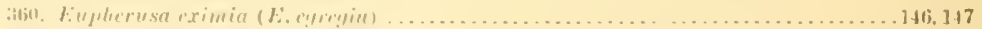

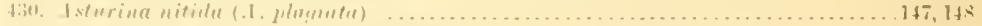

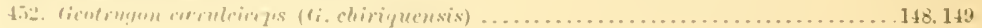

54.

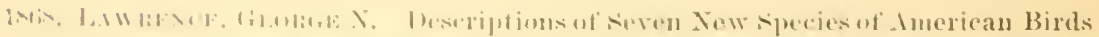

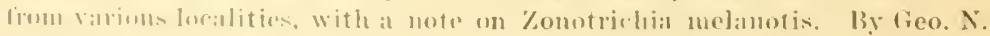

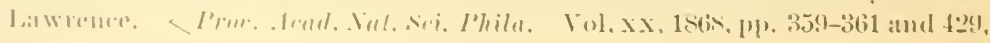
$1: 11$.

l'age.

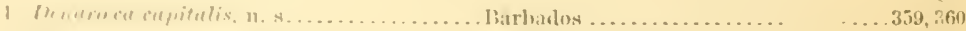

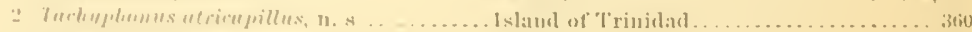

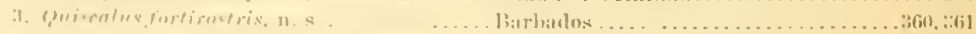

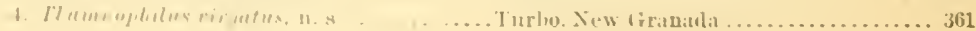




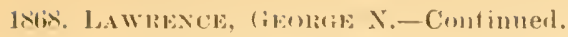

I'age.

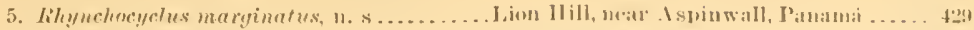

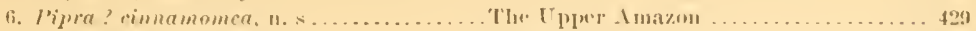

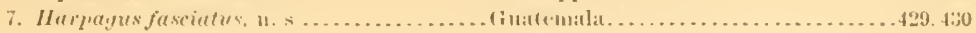

\section{Nori:}

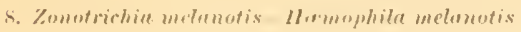
1:30

55.

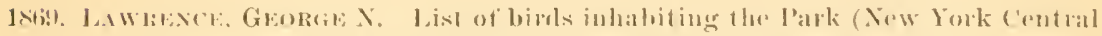

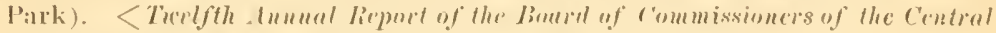

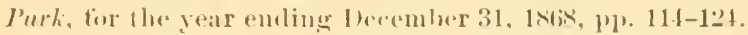

Mr. Lawreme here is anlunily simply for the nomenclature.

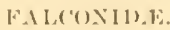

1hupotramehis columburius (Linn) - Tage.

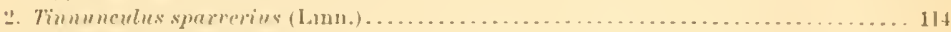

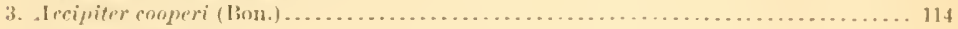

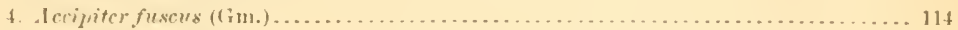

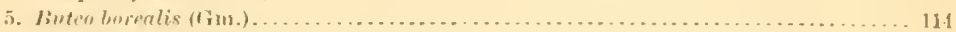

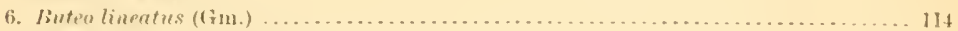

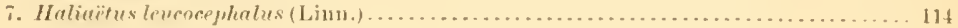

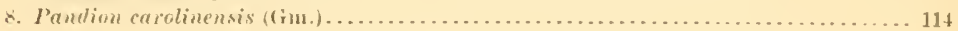

\section{STRIGI1).E.}

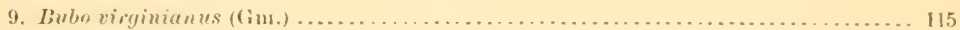

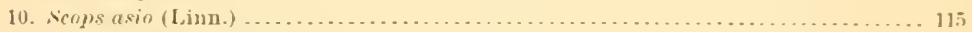

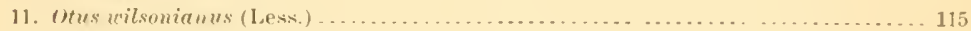

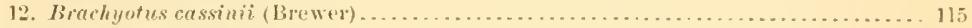

13. Viveted nirere (1)

I'I 'I'LIITE.

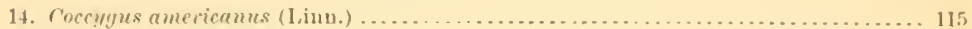

15. Cocengus crythropthalmus (Wils.) ................................ 115

PICII,F.

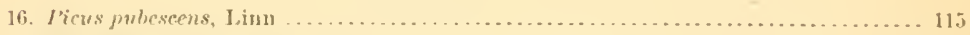

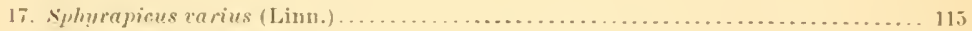

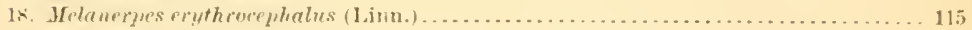

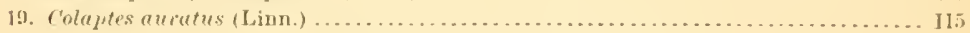

TIRO('IIII,ILE.

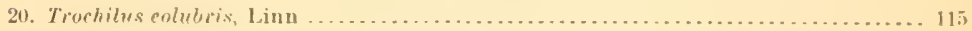

('TPSELIT).E.

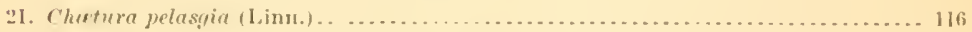

I.ATRIMILLGID.E.

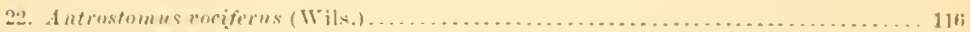

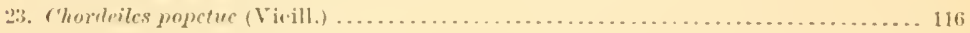

Al.'EDISII).1:

24. re'ryle alcyon (linu.)

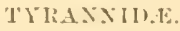

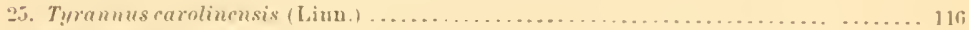

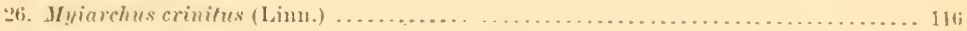

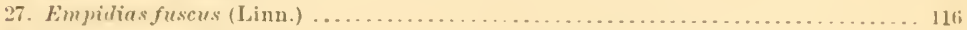

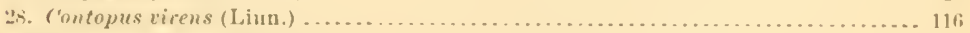

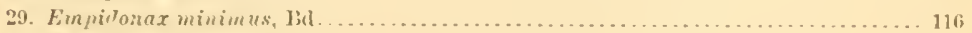

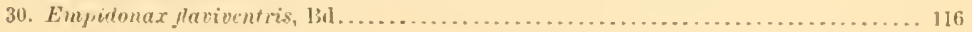




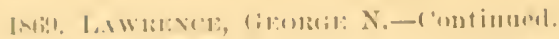

'I'IIII).

31. Trurolus mustelinus, Gim

:2. Turelus jullusio (1':als.) ...

il Turelus mimeforius, I,im

$\therefore A X I 1) 1,11) 1 \%$

35. Nulia sialis (1.inn.)

SII IIII, I:.

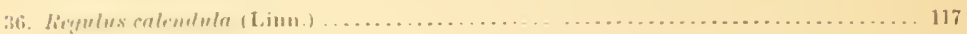

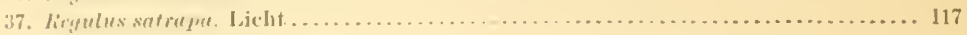

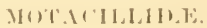

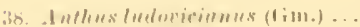

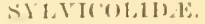

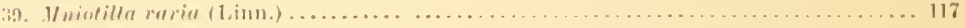

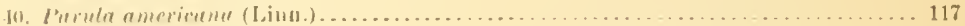

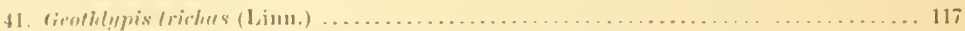

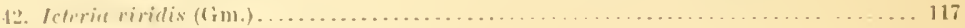

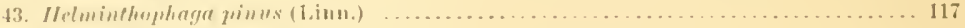

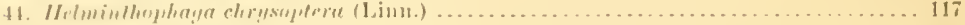

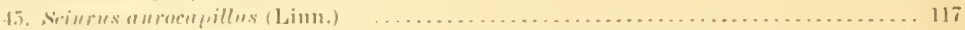

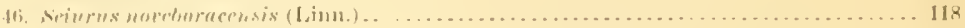

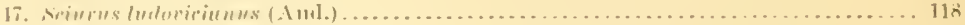

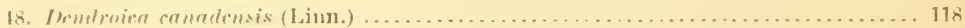

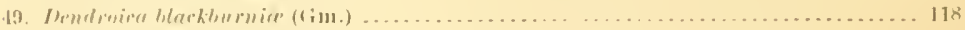

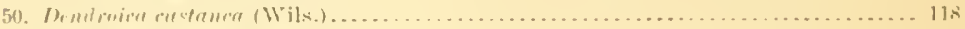

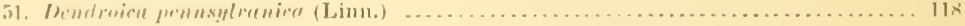

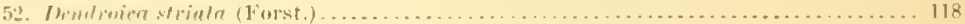

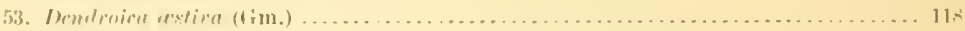

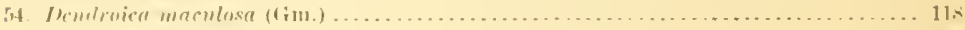

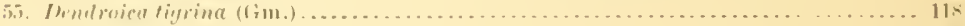

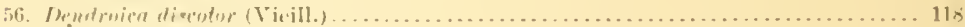

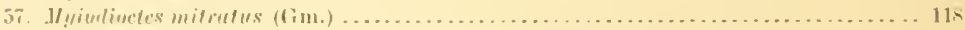

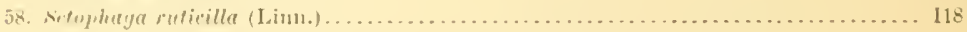

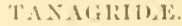

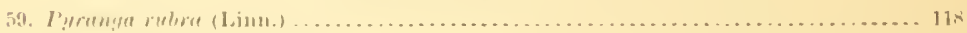

\section{IIIILXIINII)}

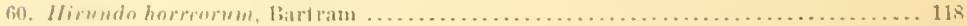

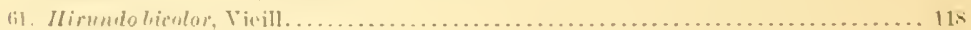

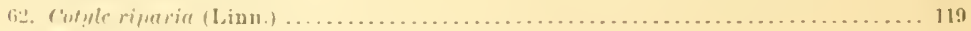

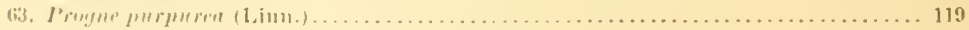

I.I ['E:L,II].1:.

61. Impelis robromem (Viejill.)

1.A.11 1.1.

lii. Collurio boretelis (Vieill.)

\section{Y IIEUXก.T}

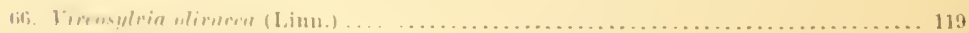

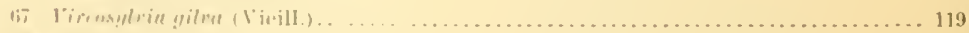

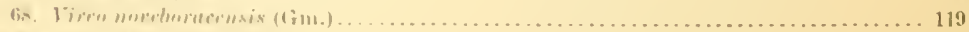

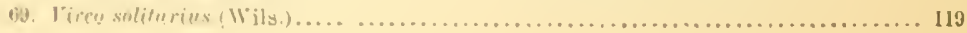


1869. LAWRENCE, (iEORGE N,-C'untimuel.

TULIIIF.

70. Mimus polyglottus (Linn) Page.

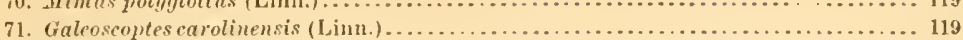

72. IIarporhynchus mufus (Limu.) ................................... 119

TKO(L)IIT1DA.

73. Troylodytes ä̈don, Virill ....................................... 119

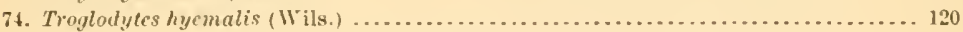

('ККНИU.Е.

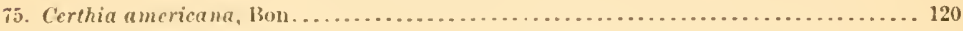

PARIDE.

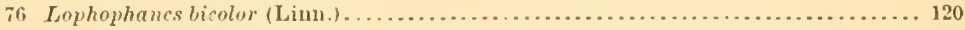

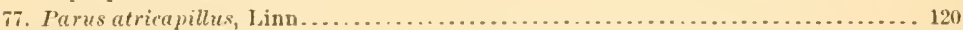

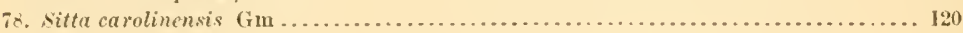

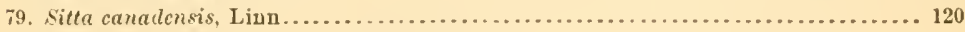

A I.AT'DIDE.

80. Alanda arvensis (Linn.)

FIINGILLID.E.

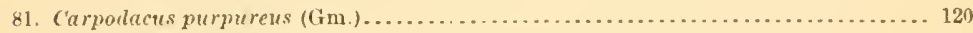

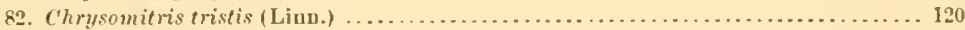

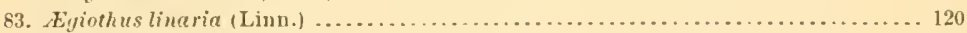

84. I'lectrophanes vitalis (Lim.) ........................................ 120

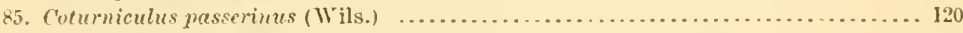

86. Zonotrichia leucophrys (Forst.) ....................................... 120

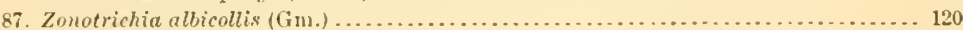

88. Junco hyemalis (Linn.) ............................................ 121

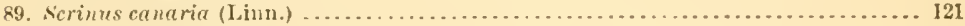

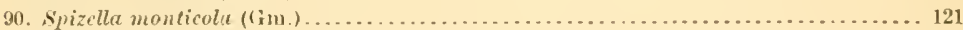

91. Spizclla pusilla (Wils.) . . . . . . . . . . . . . . . . . . . . . . . . . . . . . . 121

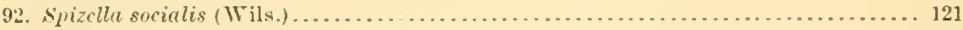

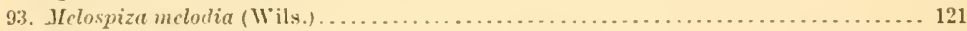

94. Melospiza malustris (Wils.) . . . . . ............................... 121

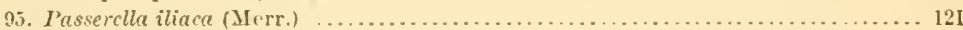

36. I'asser domesticus (Lim.) ....................................... 121

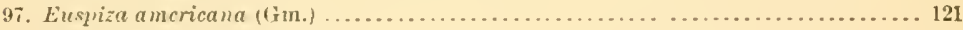

98. Cyanospiza cyanea (Linu.) ........................................ 12I

1(TERIDE.

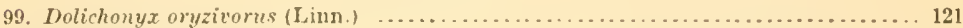

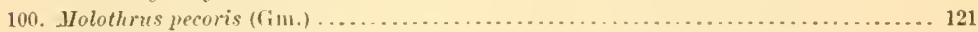

101. Agelaius phon iceus (Lim.) . .................................... 122

102. Sturnella magna (Lim.) ......................................... 122

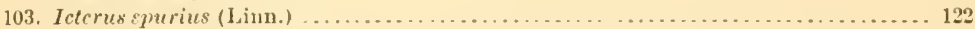

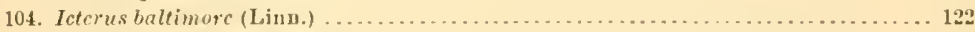

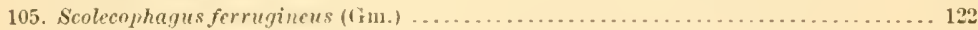

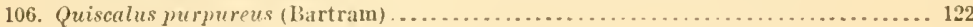

COLVIDE.

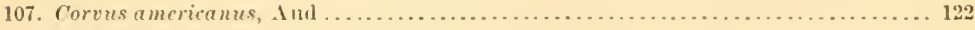

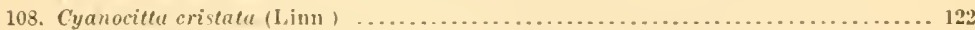

('()LUMPII).E

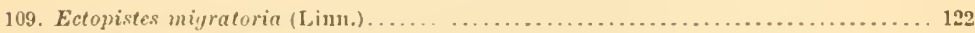

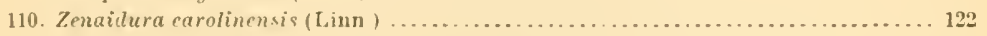

TETIAONIDE.

111. Bonasa umbcllus (Linn.) ........................................ 122

Billl. 40, N. MI.-1 


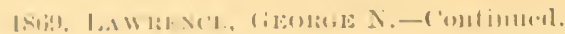

PEIIIIIH, I:

11:. Urly.r viruiniunus (1,im)

AliNFII)E

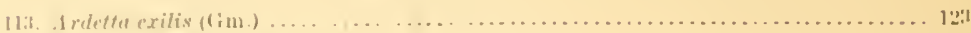

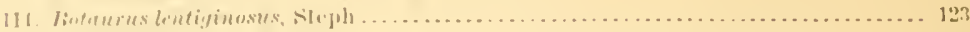

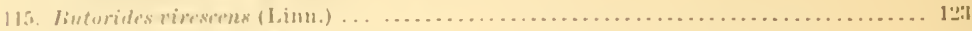

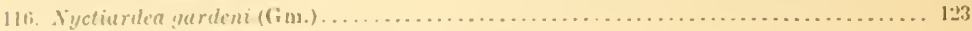

\section{I'IARADRIIJ.}

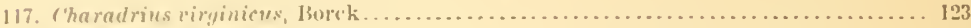

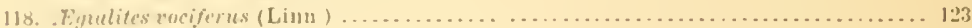

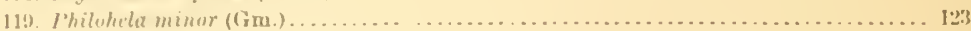

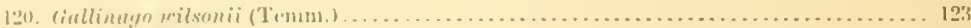

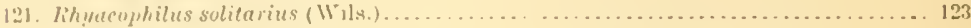

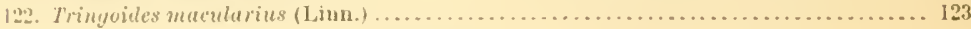

\section{RALLIIL.F.}

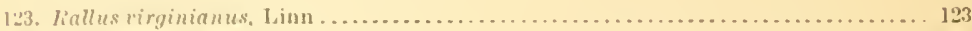

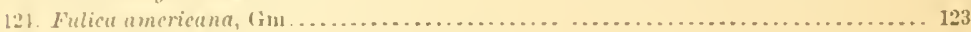

ANATIDE.

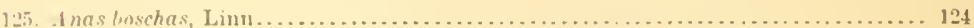

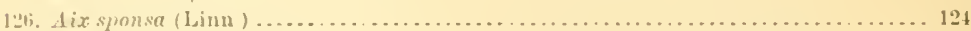

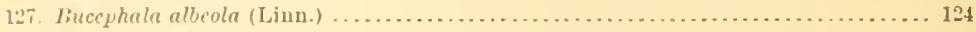

LARIDE.

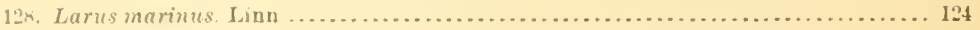

(YLIIBII) F:

129. Colymbus torruatus, Brunn 124

56.

1864. I,

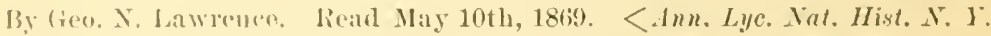

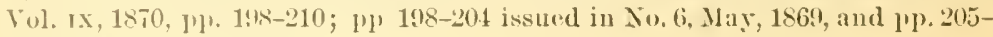
:10 in No. 7, June, 1869.

TIRDID.F

Page.

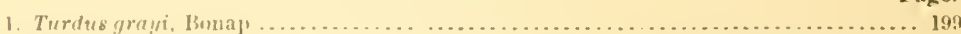

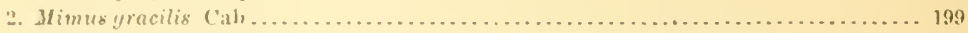

TROMLONITID.K

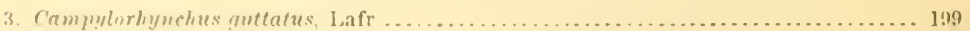

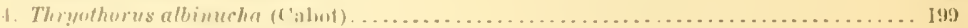

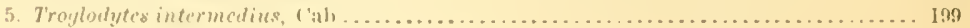

SYLVIIIS.

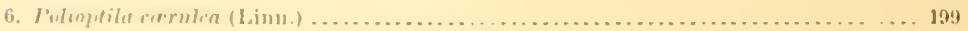

SYJICULIII:

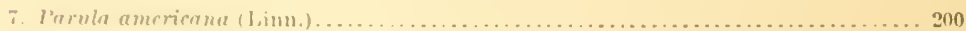

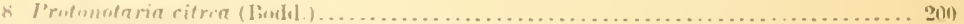

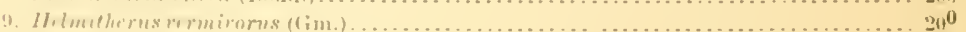

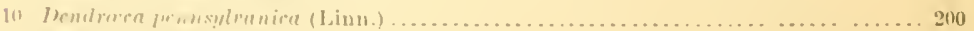

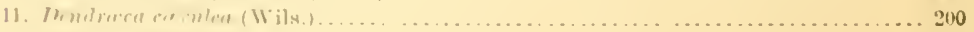

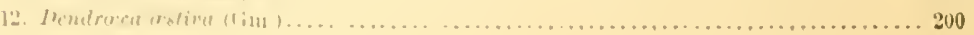




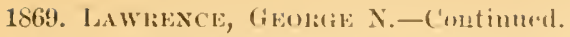

I'itro.

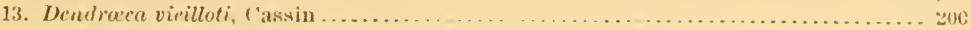

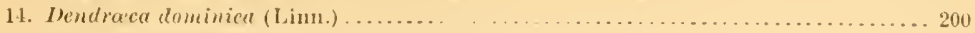

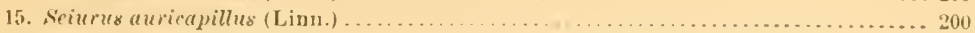

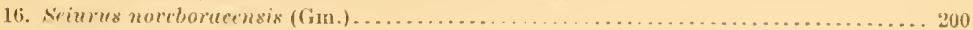

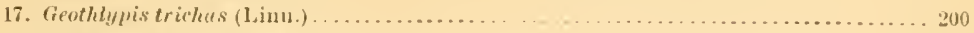

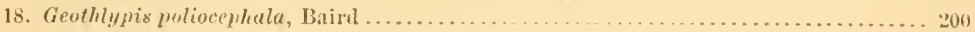

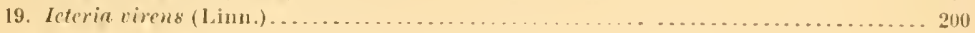

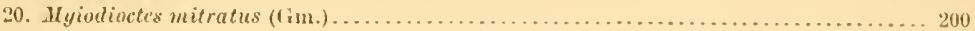

\section{IIIILNINIH.}

21. Sitelgidepteryx fulvijennis (Sicl.) 200

TIIENIIIX.

23. Cychloris davientris, Lafr... 290

TANAGIIILE.

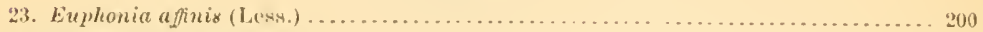

24. Saltator utriceps, Less.......................................... 200

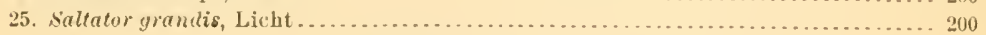

FIINGILLII).F.

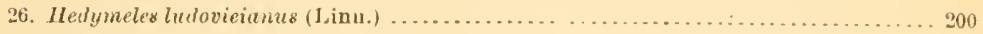

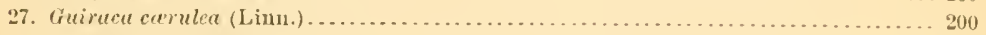

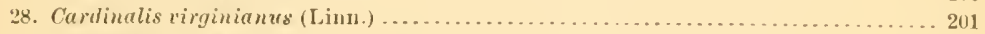

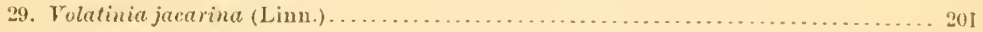

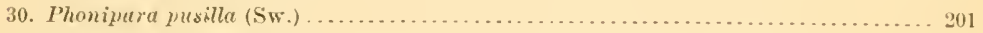

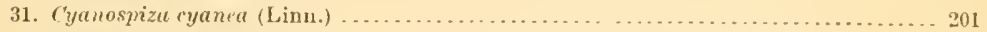

32. Cyanospiza eiris (Linn.) . . ........................................ 201

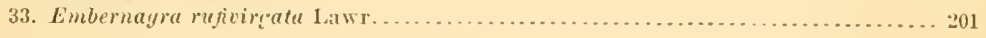

('O)IVI1).E.

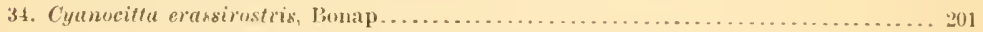

35. Cyunocorax luctuosur (Less.) . ...................................... 201

IEXIDUCOLAI'TID.E.

36. Dentromis eburneirostris (Less.) . . . . . . . . . . . . . . . . . . . . . . 201

FURIII:A IRID.F.

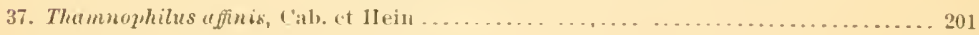

TYRINIIIA.

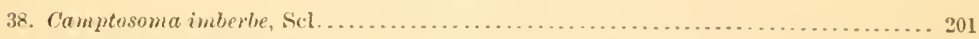

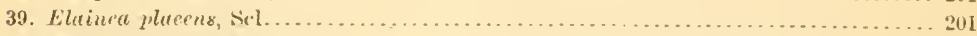

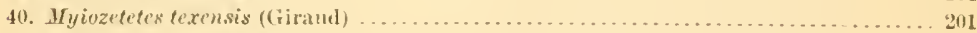

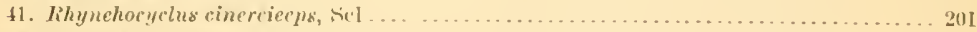

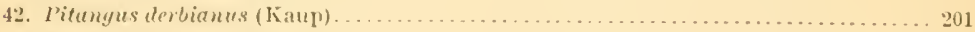

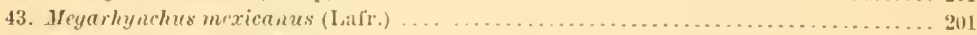

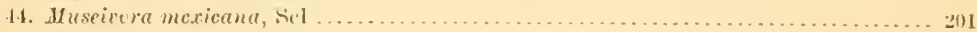

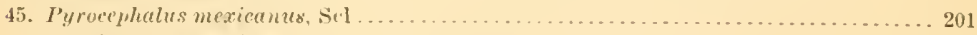

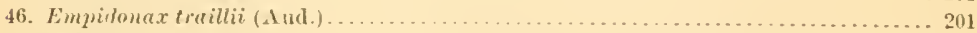

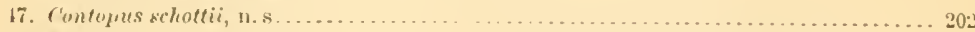

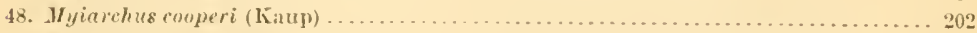

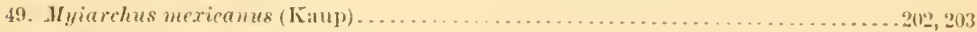

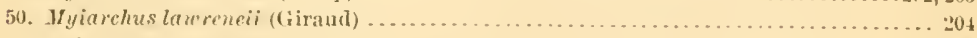

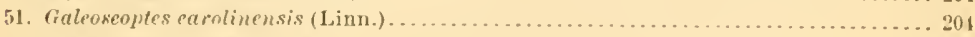

52. Tyranus satrapa (Licht.) ........................................ 204

\section{COTINGIIIA:}

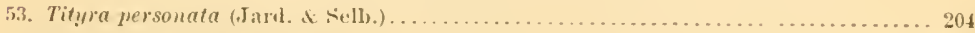

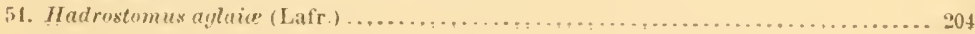




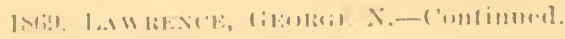

.III.IITII).F:

I'ago.

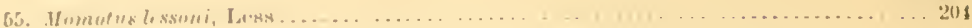

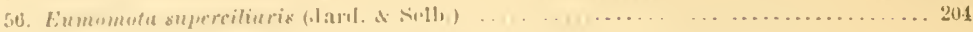

II. IEIIIII. E.

57 frryle sumerciliusio (limu.).

('AI'IIIMITLAIII,I:

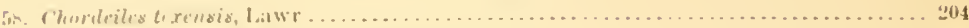

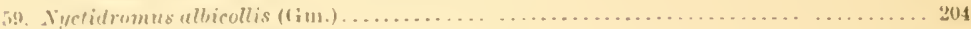

('SISELI,II).

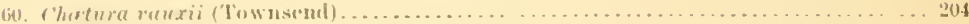

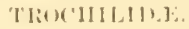

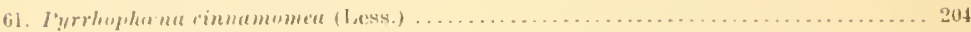

(1TILIII.

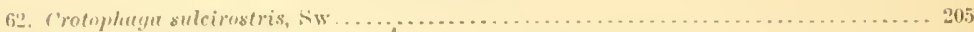

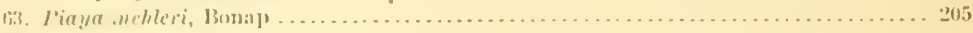

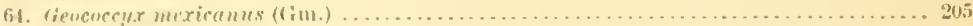

1'11'II).1:.

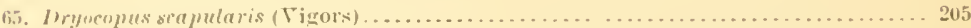

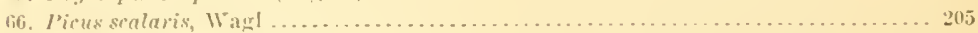

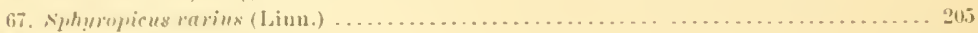

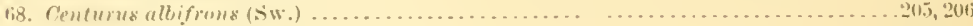

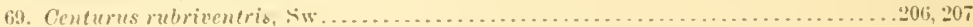

PIITACIIDE.

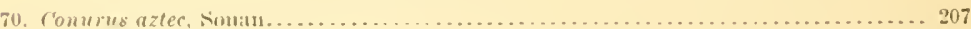

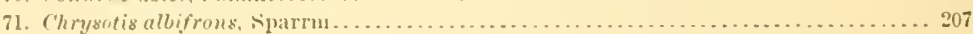

\section{S'TlitgII).}

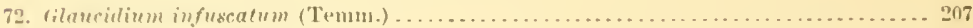

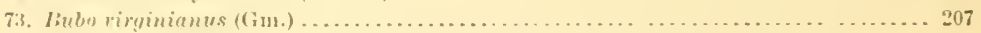

FALCONIIIE.

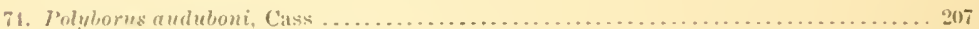

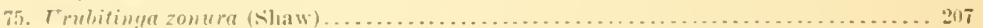

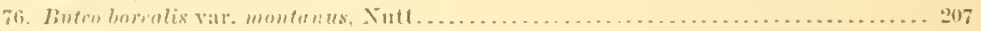

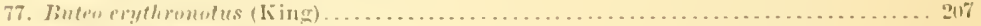

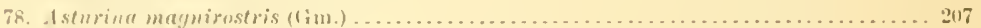

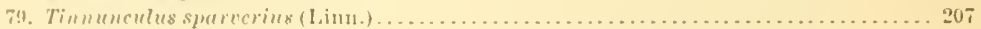

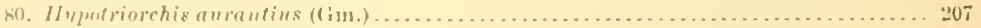

\section{(')1. ['MBII)}

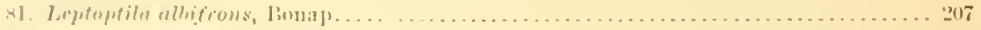

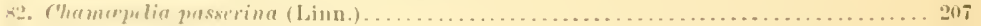

43. Cham

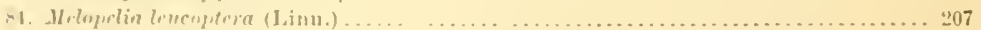

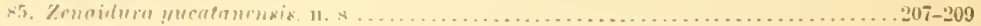

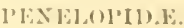

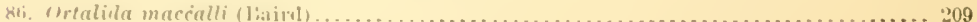

1'1:1:111']1).E.

*7. Hifur ni runulares (timuld) 
PUBH,ISIED WRITINGS OF GEORGE NEWBOLD JAWRENCE. 53

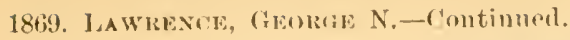

('HARIITRIIN.T:

Pagre.

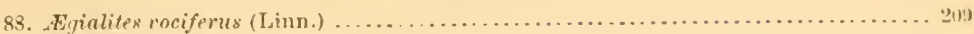

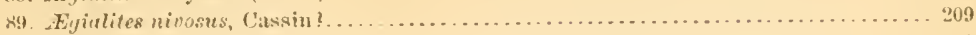

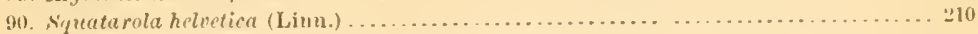

II. ENA TOI'OI)1 I.F.

91. IIamatopus yalliatus, T'rmun.

\section{SCOLORTURIII.E.}

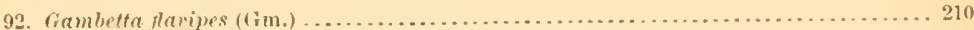

93. Calielris areneria (Linn.) . . . . . . . . . . . . . . . . . . . . . . . . . . . .

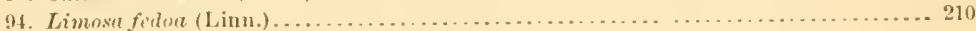

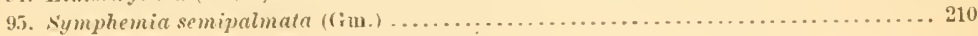

ARIEIDE.

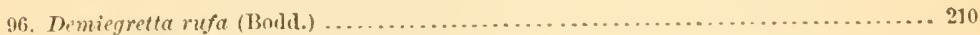

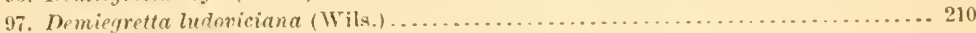

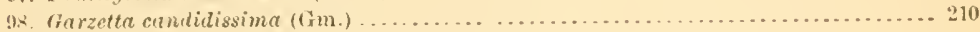

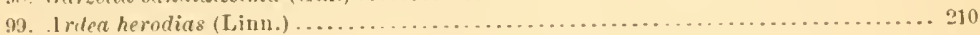

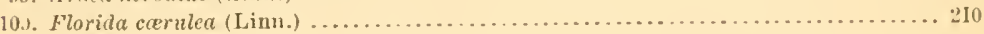

ANATIDE.

101. Fulix affinis (Forster) 210

L.A IIII).E.

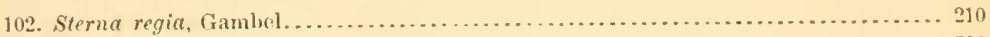

103. Sterna acuflavila. Cabot .......................................... 216

57.

1869. Idwrexce, Georef N. Catalogue of Birds from Puma Island, Gulf of Guayaquil, in the Mnsenm of the Smithonian Institution, rollecterl hy .J. F. Reve. Ese. By Geo. X. Lawrence. Read Mas 10th, 1869. <Ann. Lyye. Nat. Hist. New Fork. Vol. rx. 1870, pp. 231-238; pp. 231-236 issued in No. 7, June, 1869 ; pp. 237, 238, in No. 8, Derember, 1869.

TURDIDE.

Page.

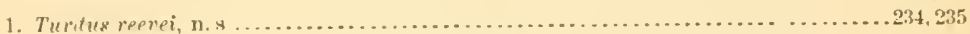

\section{TROGLODTTIDX.}

2. Thryothorus superciliaris, n, 4 . . .

\section{SILTI('()LIDD.}

3. Parula pitiaunui (Vieill.). 236

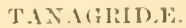

4. Tana!ra cana, sw. 236

FORMICAIIJW

5. Thromnophilus albinuchalis, Scl. 236

TYR.INNIDE.

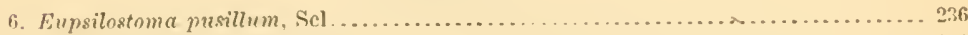

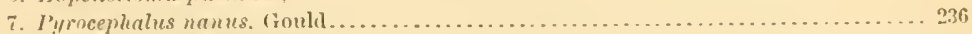

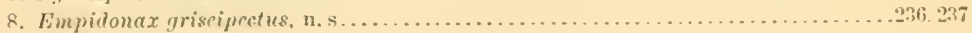

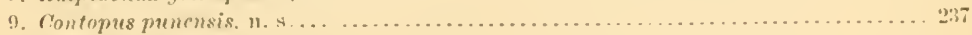

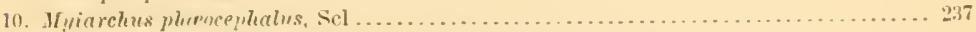

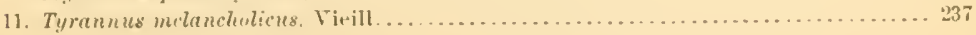




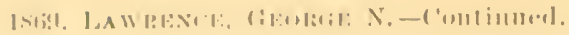

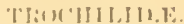

Page.

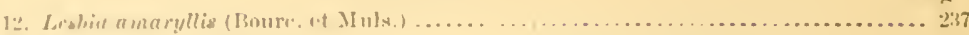

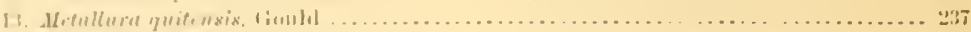

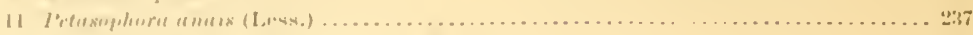

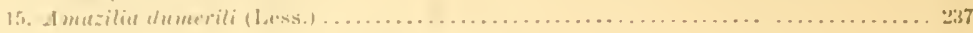

('1 (1) 111$) .1 \%$

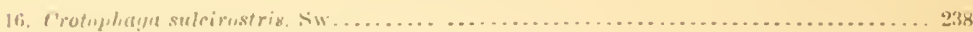

J'111.1.

17. r'hloronerpes collonotus (Waterh.). $23 x$

\section{FALCONIDE.}

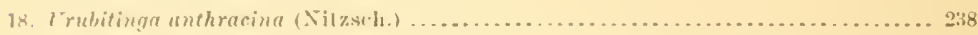

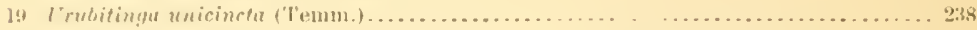

\section{('HALAJUIIU,E.}

20. . Einalilis esmipalmatus (Bunajp.) $2: 38$

RAI,III. F.

21. Jarra intermedia, Jonap' 238

58.

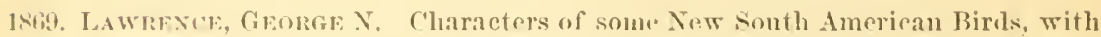

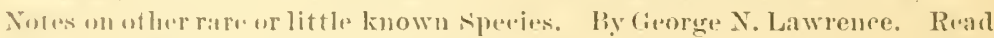

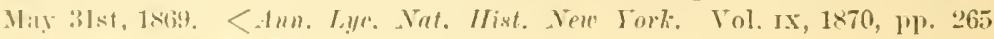
-275. [ssure] in No, 8, December, 1869?.

('HARACTERS,

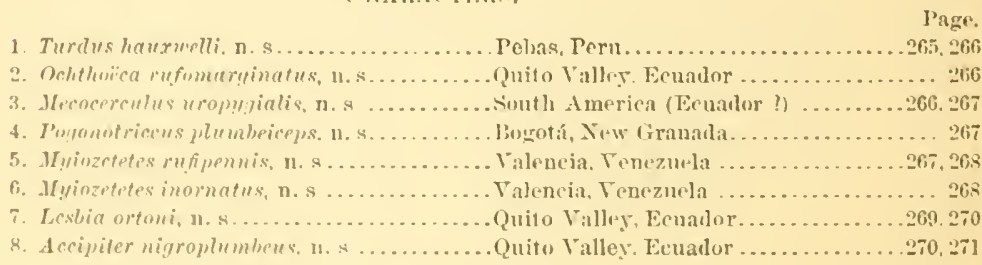

Notes.

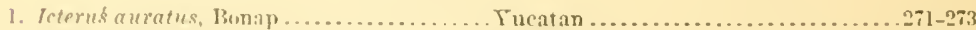

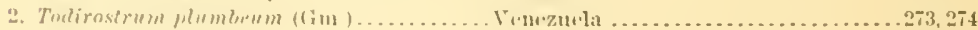

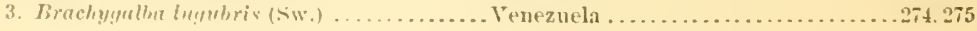

59.

1871. J.AWR

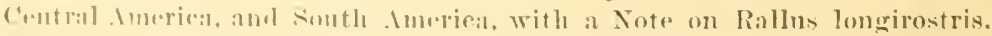

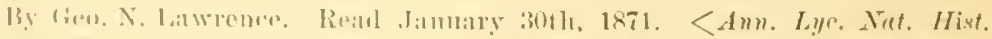

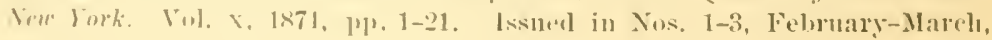
$1 \times 71$.

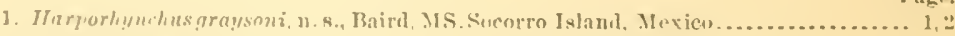

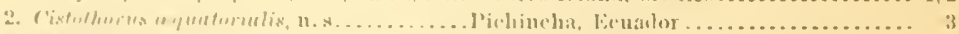

3. Trupleulules insuln

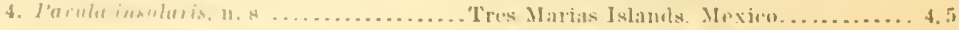

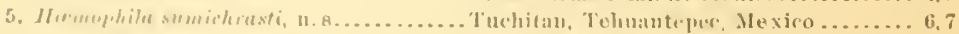

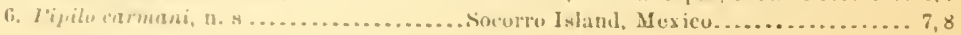




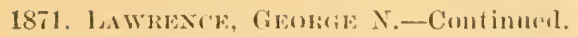

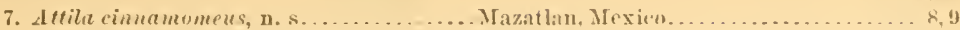

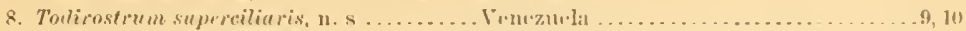

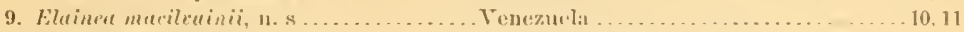

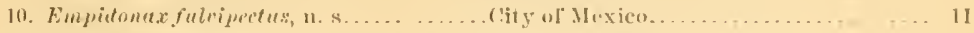

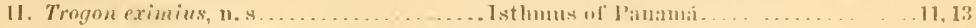

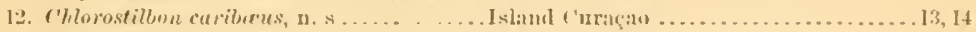

13. C'onnerus holochlores vat. bremipes, Batird,

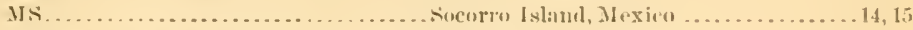

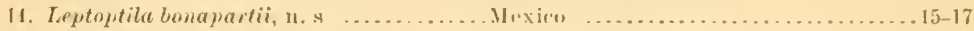

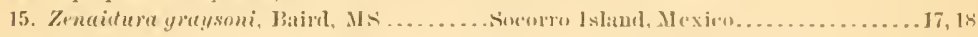

NoTL: ET⿱

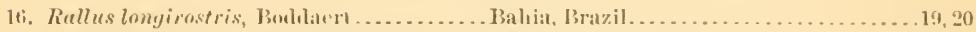

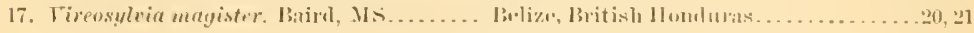

60.

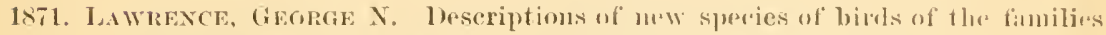

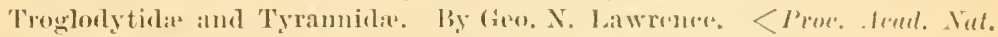

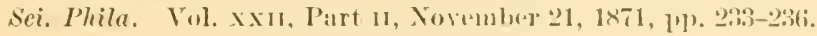

TROGIAHYTID.E.

Page.

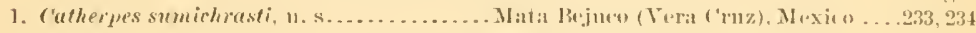

TTRANXIDE.

2. Myiozetetes grandis, n. s................ Prorince of' Tumbes, Peru ........... 234

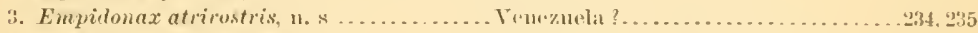

4. Myiarchus yucatunensis, n. s........... Fucatsu .....................235, 236

61.

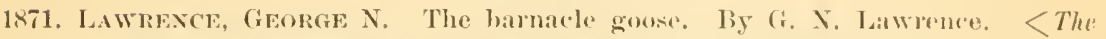

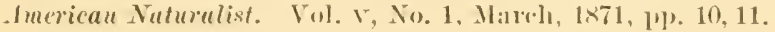

1. Brantule'ucopsis (Brelist.)

lage. C'urritu.k soumi. Nortlı f'irolina...... I0. 11

\section{2.}

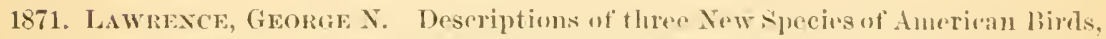
with a Noteon Eugenesspectabilis. By foro. X. Lawrence. Rearl Nov. 27th, 1871. LInn. Lye. Tat. IIist. Vew Fork. Vol. X, 1871, 111. 137-140. Issuerl in Nos. 4,5, July-Novermber. 1871.

1. Mimus niqriloris, n. s..... Page.

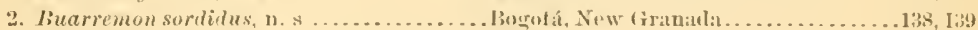

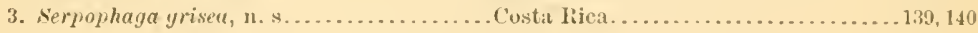

NuTk.

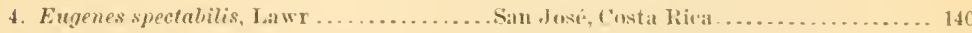

63.

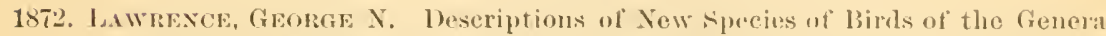

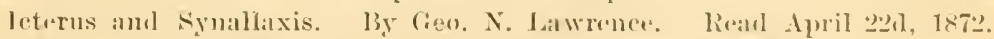

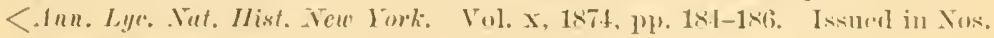
(i, 7, Mareh-May, 18т:2.

2. Sijuallaris munculatn,

Tumb's, J'erí .................... 186 
64.

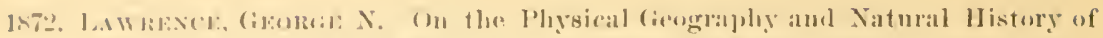

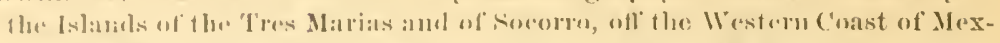

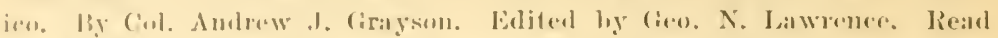

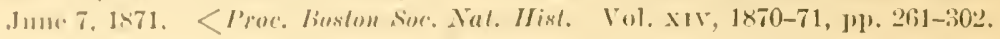

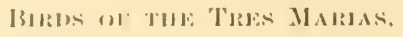

VILTERID.F.

Page.

1. ("athartesumru (Lime). 267.268

\section{H.II ( $)$ NIII.}

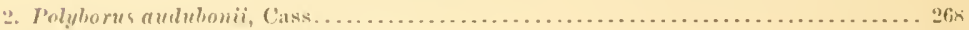

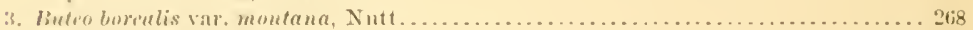

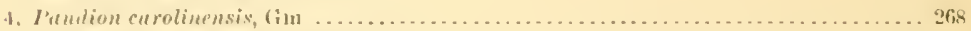

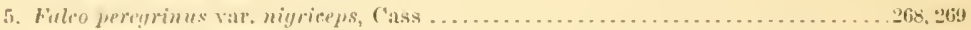

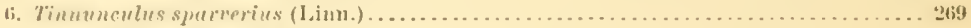

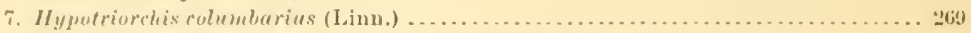

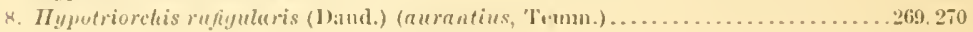

STRIGIII.

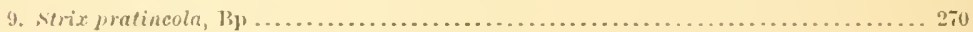

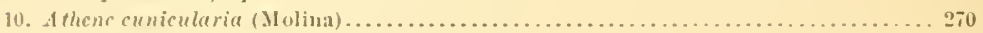

PSIT'TAC'III.E.

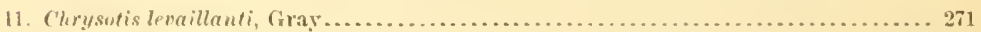

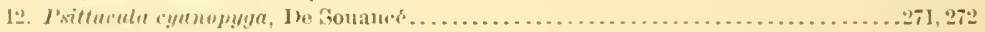

\section{TRO(IONII)}

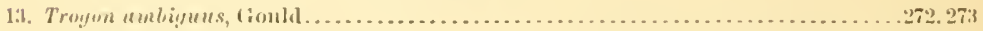

\section{(AIRIALEGID.E.}

14. Nyctilromus albicollis (tamel.)...

I'IOIDE.

15. Picus scalaris (Wagi.).

('OLITMIDA.

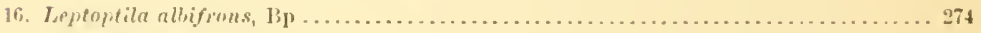

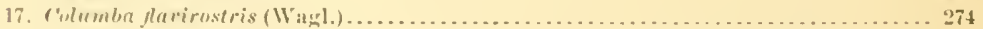

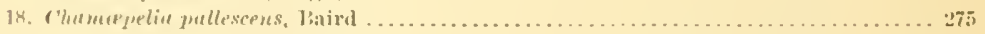

'TUJIH].F.

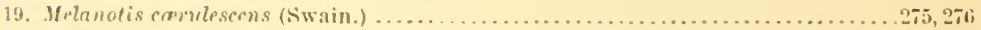

21. Turulus tluvirostris, Ṡwain . . . . . . . .

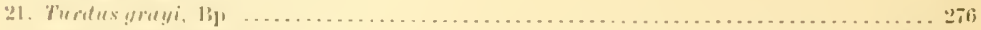

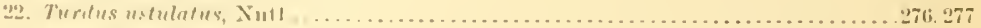

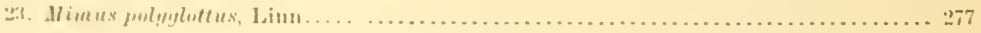

AMIELIH.E.

24. Muiwtestes uhsurus, LaJr.

MNIUTILTH.T.

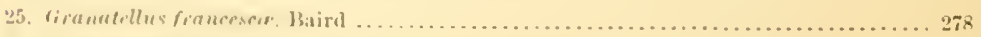

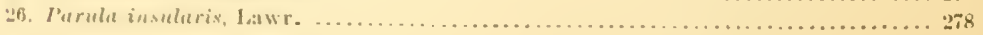


PUBLISHED WRITINGS OF GEORGE NEWBOLD LAWRENCE. 57

1872. LAWRENCE, GHORGE N.-C'ontinuedl.

TIOHLOHTTHA.

I’age.

27. Thryothores frlix, Sill 278

\section{TITINXIIS.}

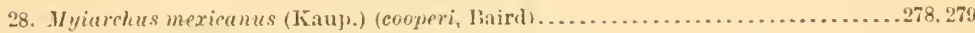

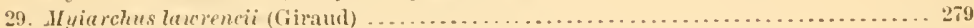

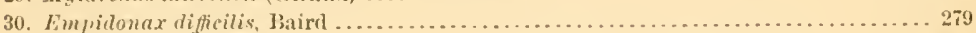

31. Elainea placens, Sel .......................................... 279

\section{('OTINGII) F.}

32. Hadrostomus aylaia: var. affinis (Eilliot) 279,280

ICTERID.E:

33. Itterus graysoni, Cassin $2 \times 0 .: 81$

TANAGIZID.E.

34. Pyranga billentata, Swain 281

VIRFONIJ)E.

35. Tireo hypochryseus, Sel 281

FIINGILIIDE

36. ('urdinalis virginiamus (Linn.) 281,282

37. Chrysomitris mexicanus, Sw 282

TROC'IILI DE.

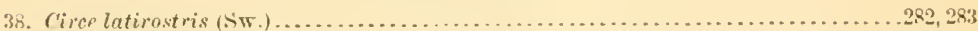

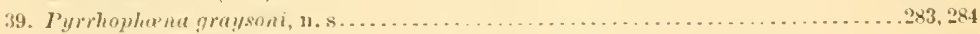

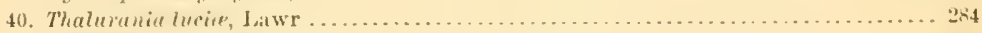

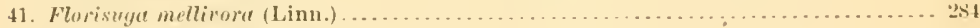

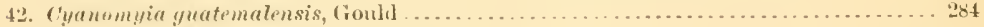

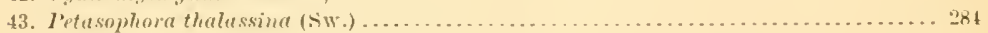

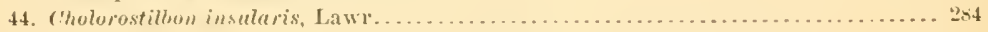

ALCEIHXIH.F.

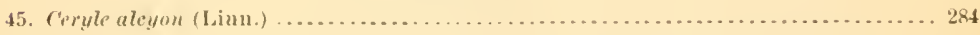

\section{II.F.II TOPOII) A.}

46. Itrematopus palliutus, T:・mm .281 .285

('II I R.A I)RIII). K.

17. Eyialitis semipulmatus (Iip.)...............

A JWHIIIE.

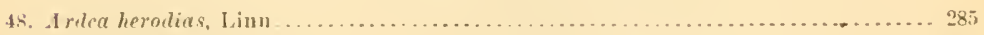

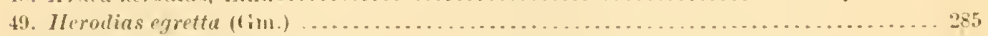

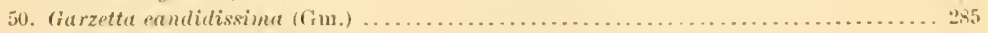

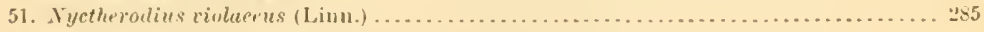

\section{I, IIII), F.}

52. IIaliplana fuliginosa var, erissalis, Baird 


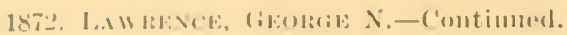

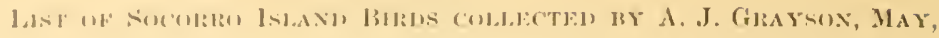
1.Nit.

IXIT'TALIILE.

Page.

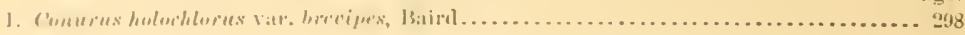

T'JIII).

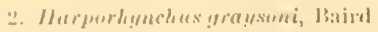
$293 \times, 299$

FIIINIII,L,I].E.

3. Pipile curmani, Lawr 299

\section{TIOGIOIITIIIA.}

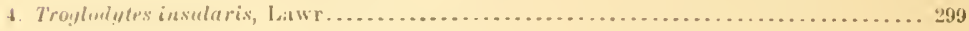

COLIMBIH.E:

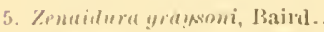

299,300

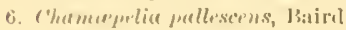
300

S'IRIOIJW.

7. Micrathene whitheyi (('onjer) 300

MNIOTLTHL.

8. Parulu insuluris, Latwr. 300

\section{FALCUNIDA:}

9. Bute' horealis vir. mondunus, Null 301

ARDEII).E.

I1). Nyctherolius violacens (Jinn.). 301

\section{LA lill).E.}

11. IIaliplana fuliynowa var. crissalis, Baird 301.302

SULII).

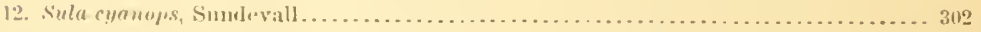

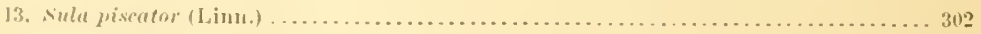

I'ELIENIIT.

14. Pelecunus fuscus: Liun 303

\section{5.}

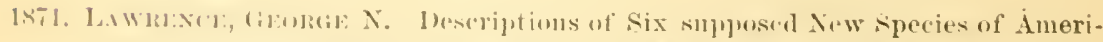

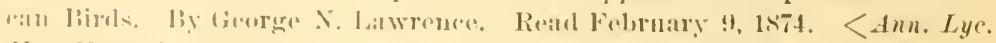

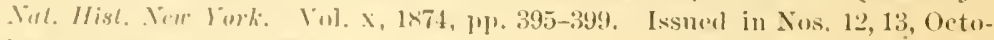

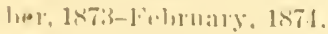

l'age.

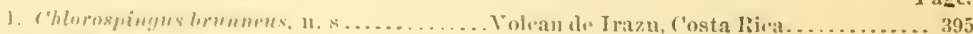

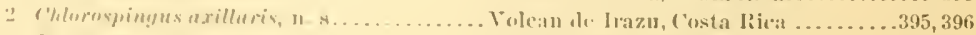

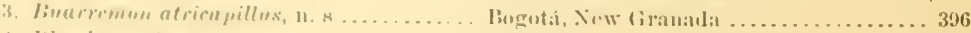

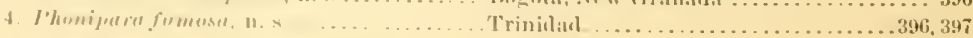

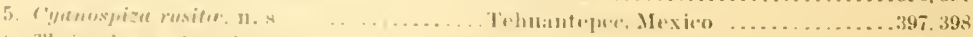

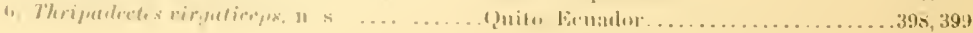


66.

1874. Lawrexes, Geores $\mathrm{N}$. The Birds of Western and Nortlowestern Mexico, based mpon colleetions male by Col. A. J. (arayson, Capt. J. Xantus, anel Ferd. Bischoff, now in the Misem of the Sunthenian fustitntion, at Waslington, 1). ('. By (ico). N. Lawrence. KMemoirs bostone Soc. Nat. Hist.

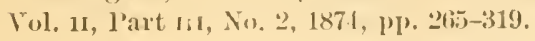

TLIRDIIIE.

Page.

1. Turdus ustulatus, Nutt .............Tres Marias .................. v6it

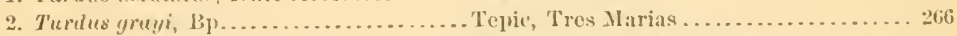

3. Turdus flavirostris, sw................. Mazatlan, Tres Marias, Plains of Colima. 2tifj

4. Ifelunotis corulescens (Sw.).............. Matzatlan, Tepis, Tres Marias, Plains of (ulima .......................... 20 .

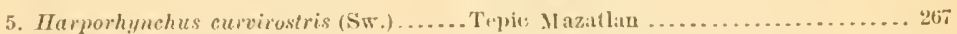

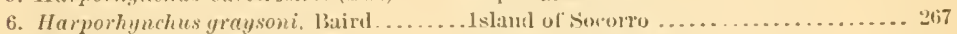

7. Mimus polyglottus (Linn.) ............ Iazatlan, Trey Marias............. 267

\section{SAXIT)LIDE.}

8. Sialia mex:ana, sw ... Mazatlan, Plitins of C'olima 267

\section{STLTIID.E.}

9. Potioptila cervulea (Liun.) . . . . . . . . . . Mazatlan, Tonila 267

10. P'olioptila nigriegss, baird Mazalau, Tejuic

TROGLO])YTID王.

11. Rhodinocichla rosed (Less.) ............ Maza1lan, Sierra Madre............ 267

12. Campylorhynchus humilis, sicl . ........ Mazatlan, Colima ................ 267

13. Cutherpes mexicanus (Sw.) ............ Tepic, Gnalalajura ................ 268

14. Thryothorus felix, ...] ................ Mazatlan, Tres Marjas............... 268

15. Thryethorus sinulou, laimd ............ Mazatlan, sinaloa, Colima........... 5268

16. Thryothores bevickii var. leucogaster,

Gould ...................... (tuatalajiari ................... 268

17. Troglodytes insula ris, baird .......... Island of 'socurro............... 268

18. C'istotherus palustris var. paluelicola. bairil........................... Mazatlan . 268

\section{MOTACILLID.F}

19. Anthus lutovicianus (1;m.)............ Mitzal lau 268

\section{SYLVICOLIDE.}

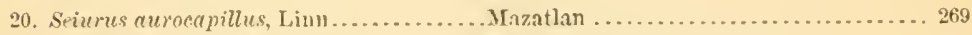

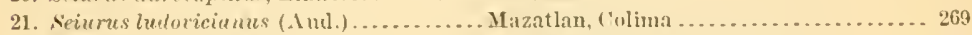

22. Mniotilta variu (Limu.) ............... Mazatlau, Colima ................... 269

23. Pamula insulavis, latwr............... Island of Socorro, Tres Marias......... 269)

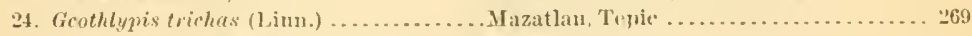

25. Geothlypis poliocephelu, liaird ......... Mazatlan .................... 269

26. Vipothlyy,is philadclphia var, macyiltorayi

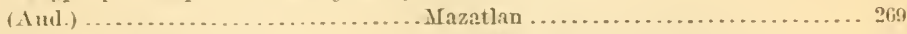

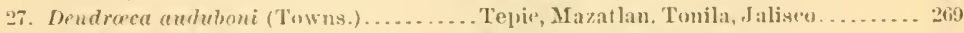

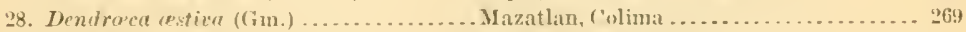

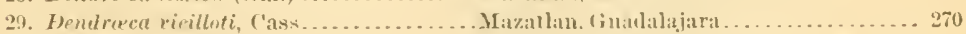

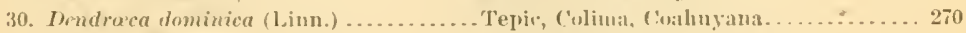

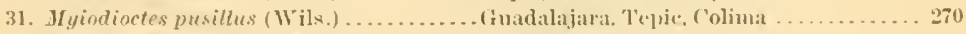

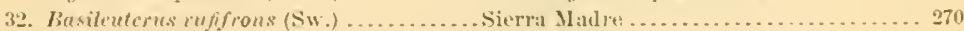

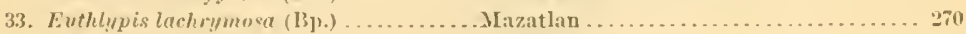

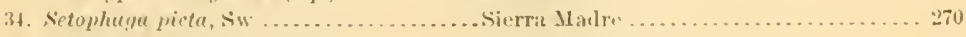

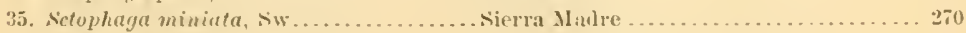

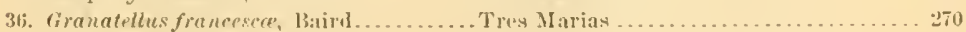

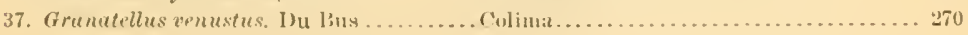




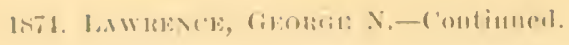

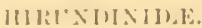

is Jerunte sulis (1.inn.)

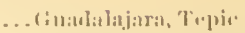

I'age.

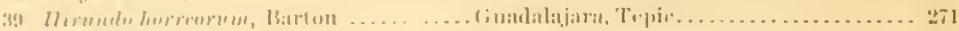

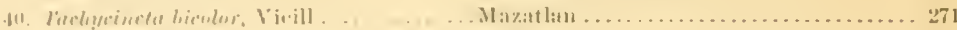

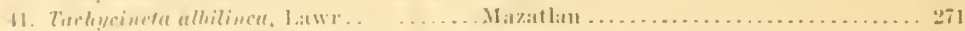

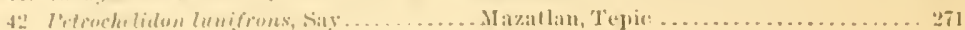

VIR⿴囗十)

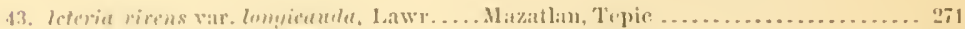

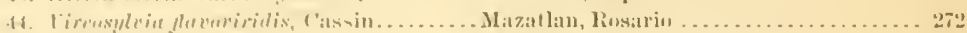

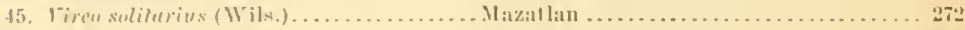

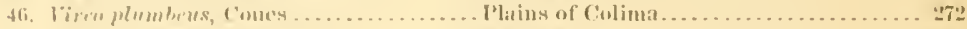

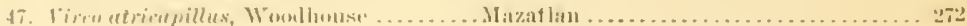

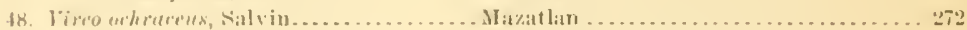

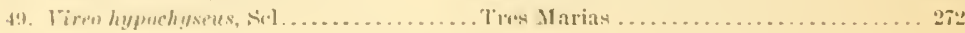

\section{L.1.IIIDE.}

50). ('ullurio ludoricianus var. "xeubiturmides,

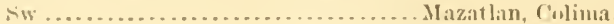
272,273

AMIELIN.F.

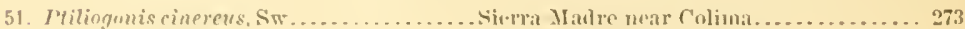

52. Myiadestes obscurus, Lat'r ............Tunila, Jalisco, Tres Marias .......... 273

TAXAGIII) E.

53. Fuphonia affinis (Less.)..............Mazallan, Nierma Malre........... 273

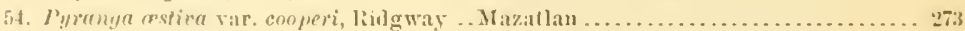

5j. Pyrama bidentata, Sw. ............ Tres Marias .................. 27t

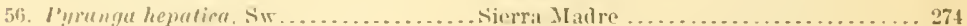

57. Sulutor plumbireps, Latwr............. Mazatlan, Tepic, Plains uf culima...... 274

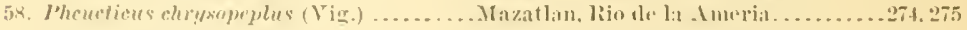

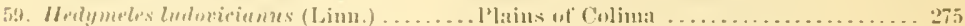

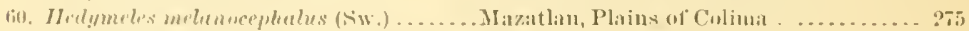

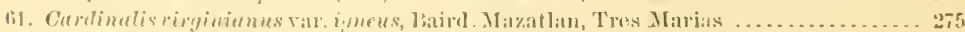

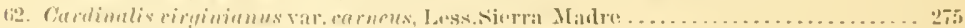

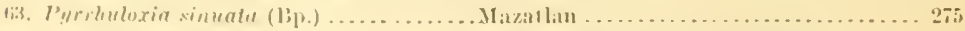

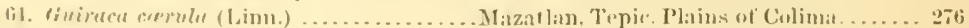

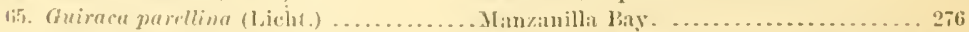

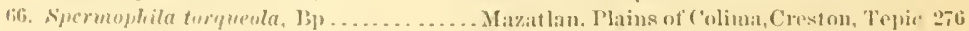

(i7. Volutinie juetrina (Linn.) ............. Mazatlan, Mazatlan River, Mountains of

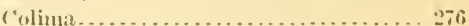

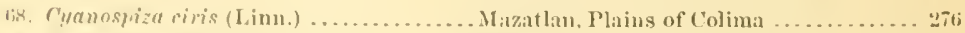

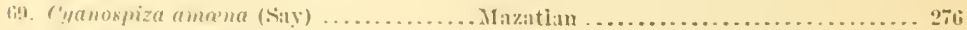

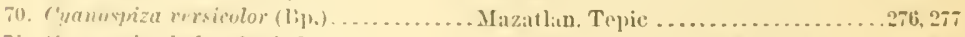

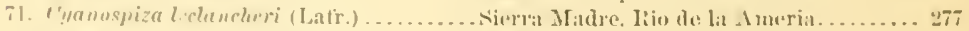

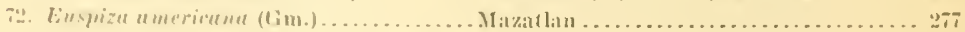

7.3. Zometriphin leweuplerys var. yetmbelii.

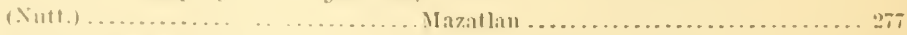

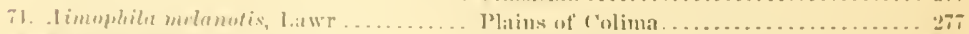

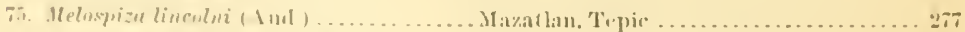

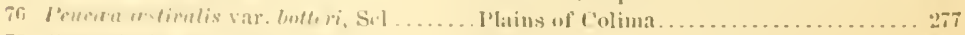

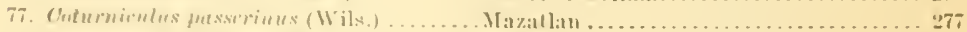

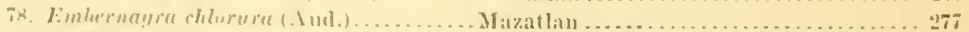

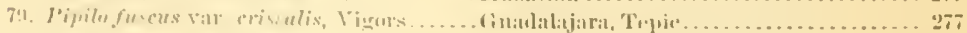

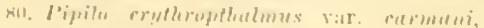

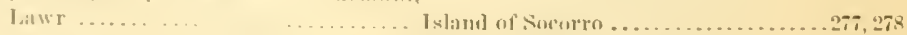

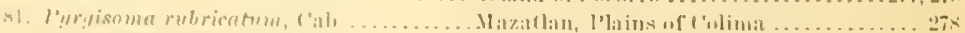

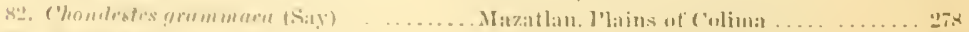

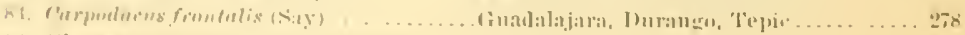

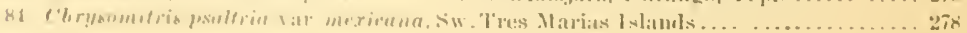


1871. Lawhisce, Grongis N.-Continued.

AHIUIH.K.

85. Otocoris alpestris var. ehrysole'mut

lage.

(W:al.) ...........................

10'TEIID.F.

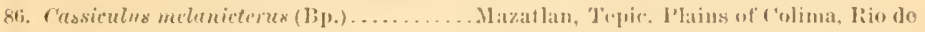

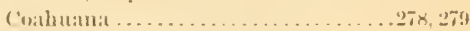

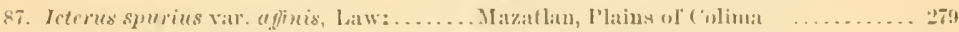

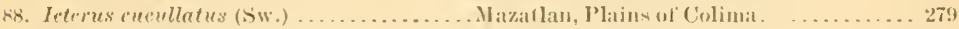

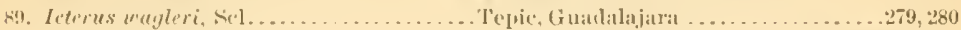

90. Ietcrus pustulatus (Wagl.) ............. T'pic, Mazatlan, l'lains of Colima, Man. \%anilla liay ......................... 280

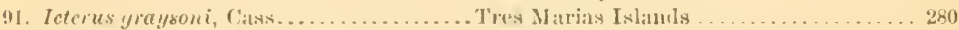

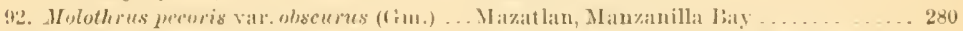

93. Julothrus eneus (Wagl.) ............... Ma\%atlan, Man\%anillat liay, Mlountains of

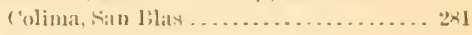

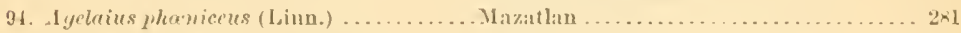

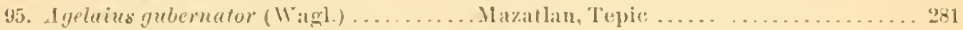

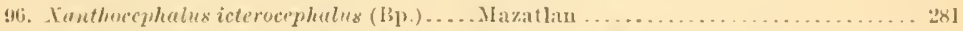

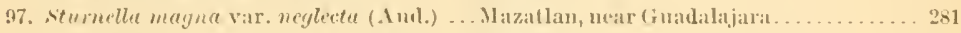

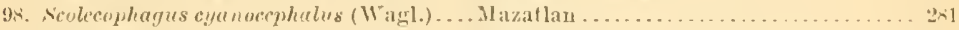

49. (Buiscalus major, Vieill................ Manzanilla Jaty, Jlains of Colima...... . 28]

100. Quisealus major var. macrourus, siw . . . inalalajara . . . . . . . . . . . . . 281

101. Quiscalus major var. pulustris (Nw.) ... dlazatlan ...................28, 28:

\section{('Olil'I P.E.}

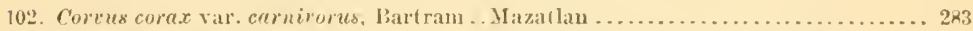

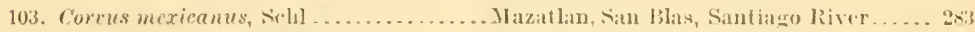

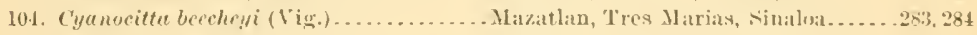

105. Cissolopha saublesianus (Latr.) ......... Plains of Colima, IIanzanilla Bay, Lat

Trochas............................ 284

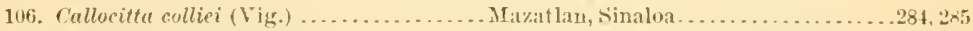

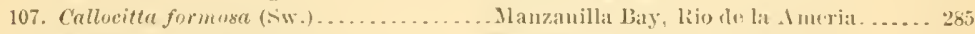

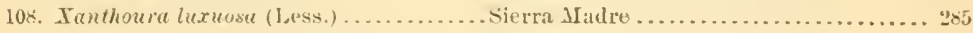

\section{DE.TIHI)('OL.APTID.E.}

109. Dendrornis mentalis, Baird ............ Mazatlan, 'Tepie. . . 285

TIRINTHIE.

110. Attila innamomous, latwr........... Mazatlan noar Tepic.............. 285

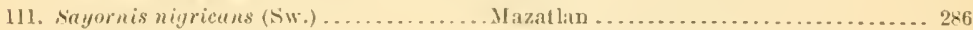

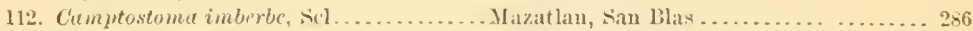

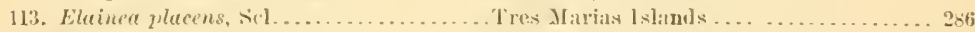

114. Myiozetetes t xensis (Girand) ........... Jazatlan. Tepir. Mlatins of Colima, Tonila. 286

115. I'itangus derbiums (Katup.)............ Mazathu. ['latus of 'olimal ........... sx6

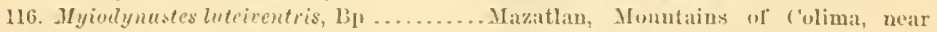

117. Pyrocephalus rubincus var. mexicanus,

sid ............................. Mazatlan. Plains of Colima, Tepic....... 28i

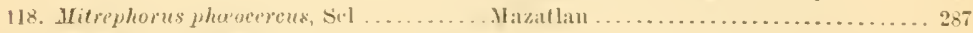

119. Empilonax flaviventris var. diflicilis,

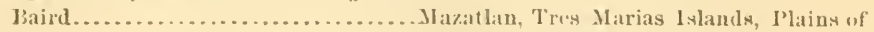

121. Empidonax traillii var. pusillus (Sw. S

Colima, Tipic................... $28 i$

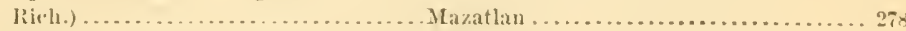

121. Contopux virens var, richarelsoni (Sw.) ..Jazatlan ...................... 287

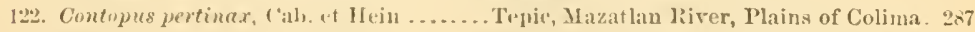

12\%. Myiurehes mexieunus (lianp.) ........... Mazatlan, T'res Marias 1slauds, Gualala.

jara, Trpir ......................287, $2 \times 8$

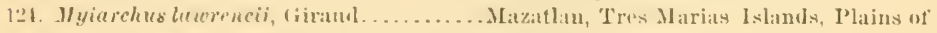

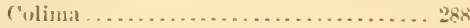

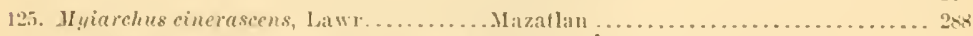

12t. Tyranus melunchelicus rar. satrapu.

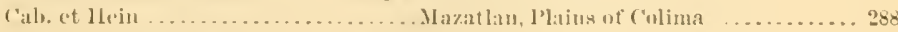

127. T!runus melanchelicus var. conekii,

bairl.......................... Inatitlau 


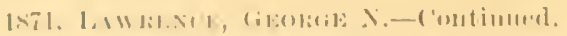

P'ine.

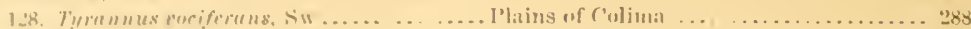

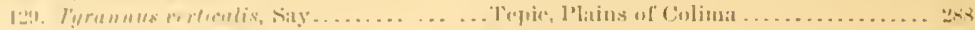

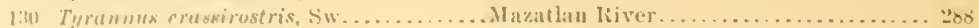

\section{(1)TISFII).}

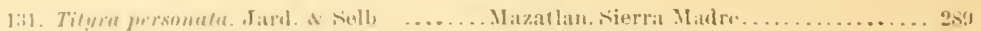

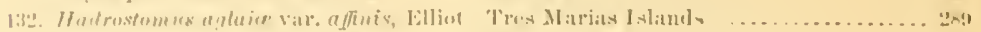

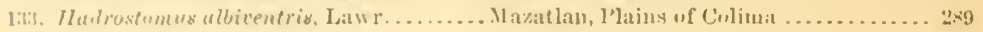

\section{M()MO'ID IS.}

134. Humu/us mixiranus, siw

Mazitlan, Plitins of ('ulima 289

\section{ALEEDIXIDX.}

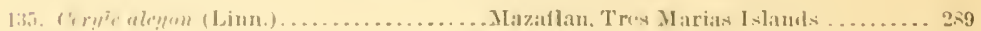

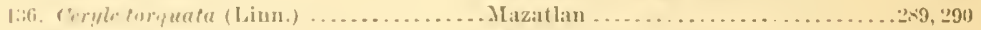

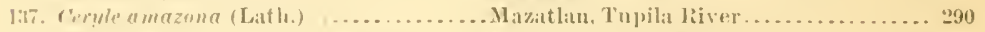

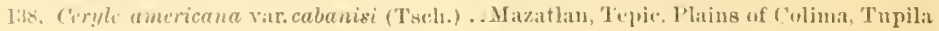

Iiver, near (juadaliajara............. 290

TROGONID)E.

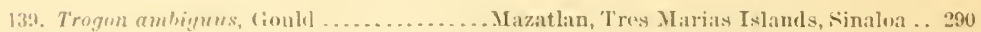
140. Tromon citreolus, (:ould. . . Mazatlan .......................200, 291

CAIRIMULGI1).

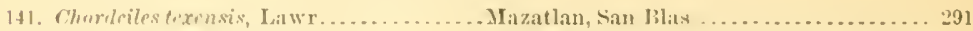

142. Syetielrom albicollis (fin.) ............ Mazatlan, Plains of' ('olima, Tres Marias

Islankls....................... 291

TlOQ:IIILID):

143. Thelurania lucux, Lawr............ Tres Marias Islands .............. 291

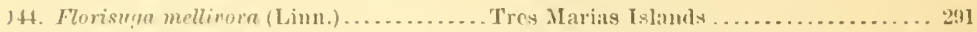

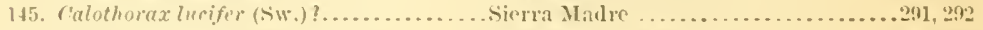

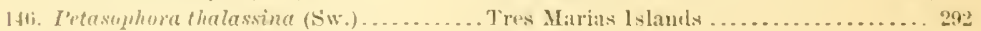

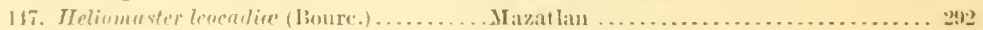

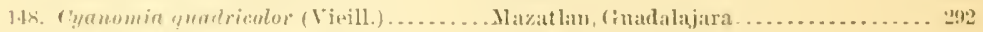

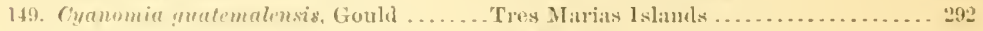

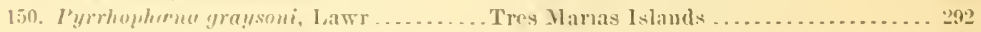

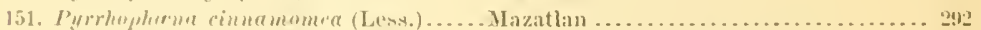

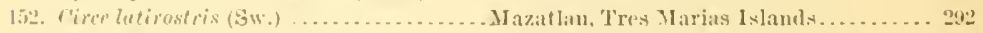

15\%. Chlorostilbon insularis, Litwr......... Tres Marias Islamls . . . . . . . . .... 29:

\section{CTCULIDA.}

15.4. C'rotophanga sulmirostris $(\mathrm{S} \pi$.$) ............ Mazatlan, Tepic, Plains of Coliına . . .292. 293$

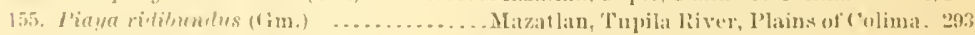

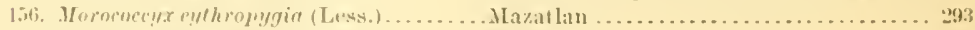

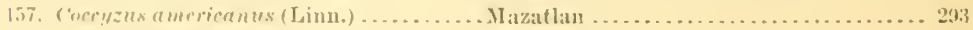

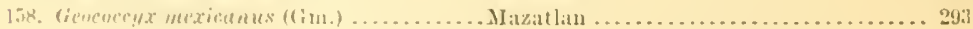

I'16113.1;:

1.5. Campryilus guatrmalensis (11artl.) . . . Mazatlan, Jio do la Aneria............ 29:3

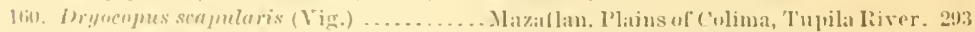

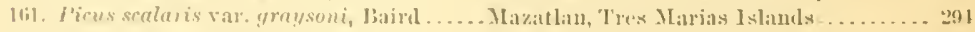

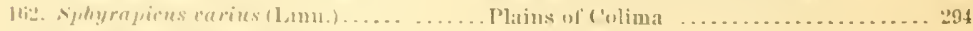

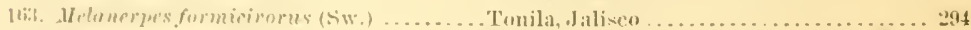

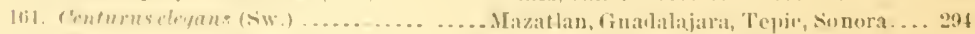

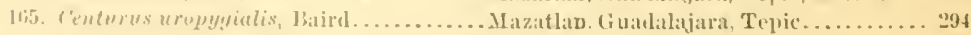

\section{I'TIT.ICID.E.}

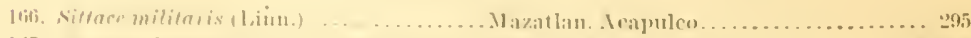

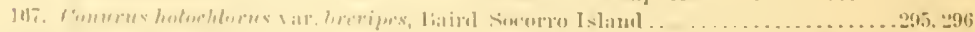

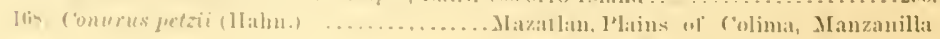

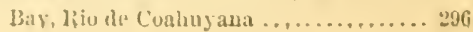




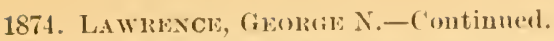

Page.

169. Chrysotis levaillantii, (iray.

Tres Marias IsImuls, Tupila liver, lio de foahuyana ..................... an

170. (hrysotis albifrons (spartm.) ......... Mazathan ...................... 996

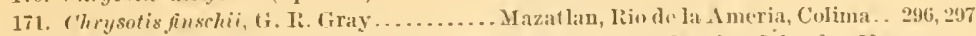
17. I'sittacula cyanopyga, De Souncé........ lazatlan, Tres Marias Islamis, Manza villa bay ....................... 297

\section{STIRIG1D.E.}

173. Speotyto eunicularia var. hypogera (Bp.). Tres Marias Islands. Mazallan ........ 29!7

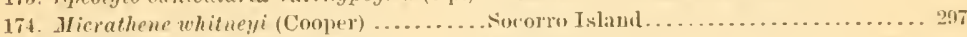

175. Vilaucidium fermginerem (Мах) . . . . . . .

176. Glaucirlium infuscatum var. grooma,

Wagl ........................ Mazallan, Colima................ 298

177. syrnium virgatum, Cass............. Mazatlan, ('olima................. 29s 178. Strix flammea var. pratincola. Blp....... Tres Mlarias Islands, Colina, lio de da Ameria............................ 298

\section{FALCONIDE.}

179. Circus cyaneus var, hudsonius (Lim.) ... Mazatlan . . . . . . . . . . . . . . . . . . 988

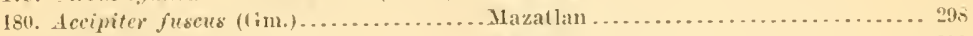

181. Aecipiter cooperi. Bp................ 1lazatlan ....................... 298

182. Isturina plagiata (Licht.) ............. llazatlam, Sinaloa . . . . . . . . . . . 298, 999

183. Jicrastur semitorquatus (Virill.) . . . . . . Mazatlan .......................... 299

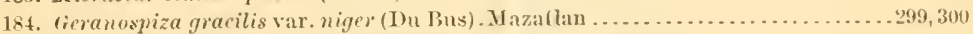

185. Herpetotheres cachimnans (Linn.) ........ Mazat lan, sunori. . . . . . . . . . . . . 300

186. Felco peregrinus var. anatum. Ijp....... Mazatlan, Thes Marias Islauds ......... 800

187. Hypotriorchis columbarius (I imu.) .... . Mazatlam, Tres Marias Islants ......... 300

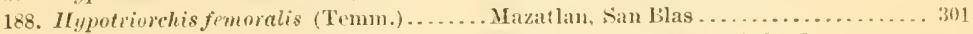

189. I! !potrinrchis rufigularis (Daud.) ....... Mazatlan, Tres Marias Islauls, San Jlas :301

190. Tinmuculus spareerius (Limu.) . . . . . . . Mazatlau. Tres Marias 1slanuls. . . . . . . 301

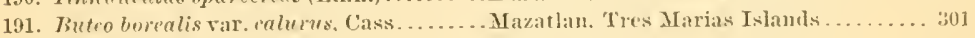

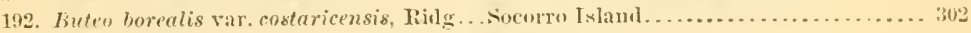

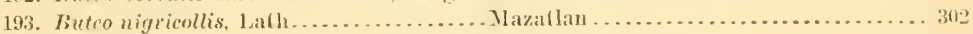

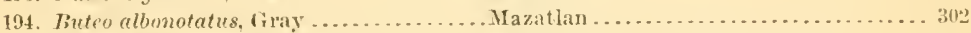

195. Craxirex unieinctus var. harrisii (Aul.) . Iazathan, Tepir, Jalisco, Colima ........ 302

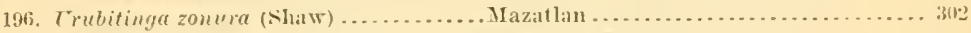

197. Trubitinga anthracina (Licht.)......... Iazatlau, San Blas................. 302

198. Iandion halië̈tus var. carolinensis (fim.) Colima, Tres Marias Islamds, Mazatlan . 303 199. Polybores thares var. auduboni. Cass.... Mazatlan, Tres Marias Islands, Colimal.. 303

\section{TULTITIIIE}

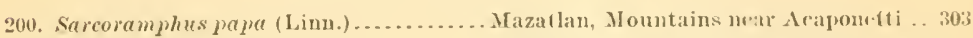
201. ('athartrs aute (Liun.) ................. Mazatlan, Tru's Marias 1stands, Colina . .303 20. Cathates atratus (1)artram) . . . . . . . . Iazatlan . . . . . . . . . . . . . ... 303,304

\section{COLIMIBII, E}

203. Columba fasciata, Say.

204. Columba flavirostivis (Wagl.)

Mlazatlan, Tres Marias Islands, Sierra Miulre ....................... 304

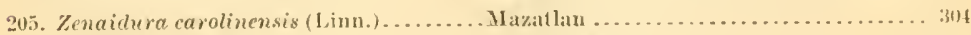

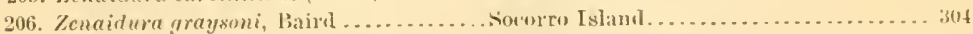

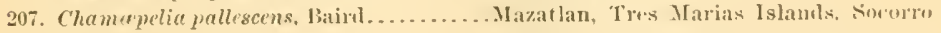

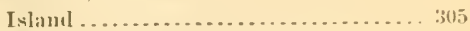

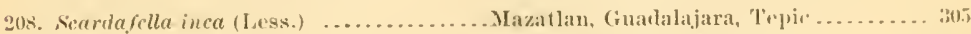

209. Jelopelie lencoptera (Limn.) ............. Mazatlan. Colima, Nonora ............ 305

210. Leptoptila allifions, Bp............... Ilazatlan. Tres Marias Islands........ 305

\section{PEXELOPIIIE.}

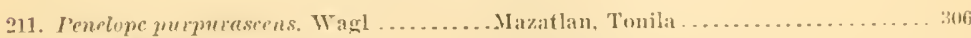

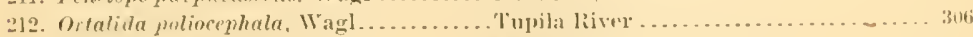

213. Ortalide wayleri, Gray.............. Alazatlan, sonora, Colina............. 306 


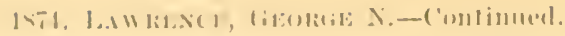

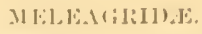

l'age.

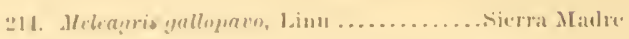
3116

\section{Plilill11).E.}

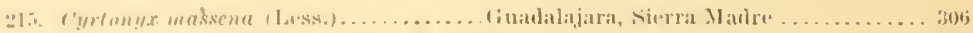

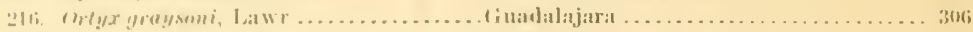

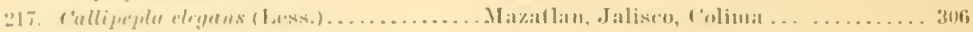

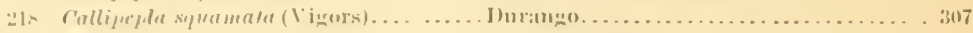

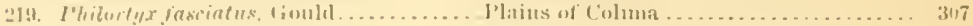

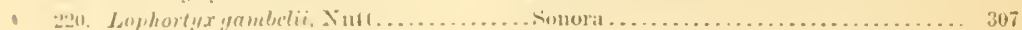

TIX.1111.K.

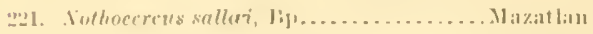
$: 07$

('II A liAl)III)T.

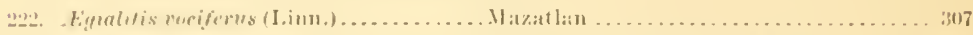

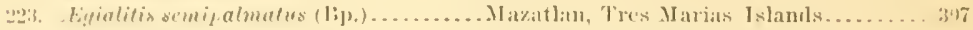

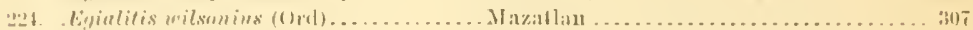

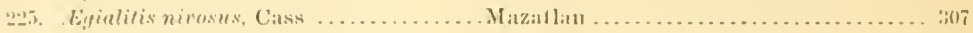

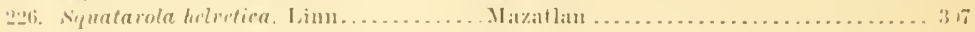

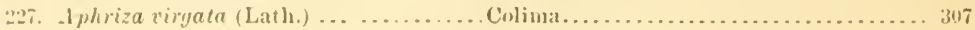

\section{1:3 I I (1) (1) II).}

2n. Homatepus palliatus. T'rmm........... lazatlan, Isabella Island, 'Tres Marias

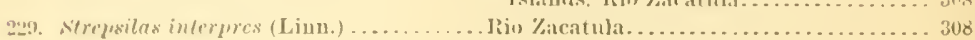

\section{IRERTRYHOSTIII)E.}

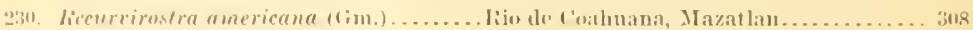

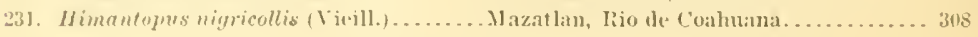

\section{S(')1, I) I'A CII). E.}

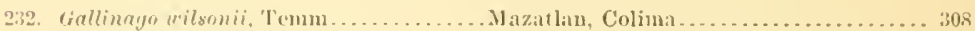

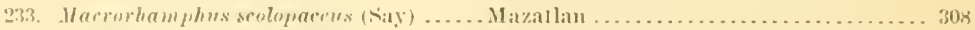

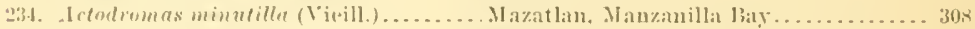

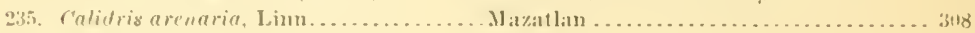

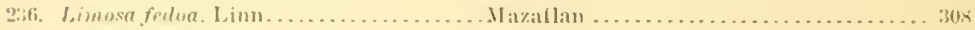

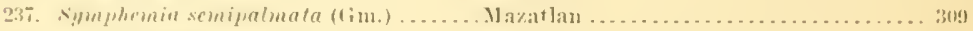

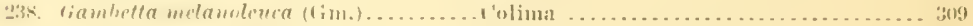

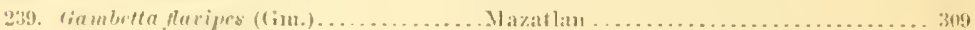

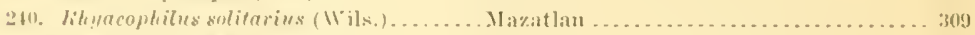

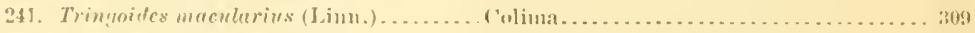

212. Dumenius lnn!, irostris, Wils......... Mazatlan ................... ;

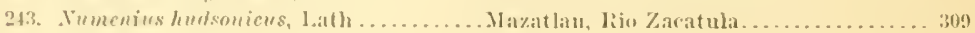

T.IXTT.AIIH.Y:

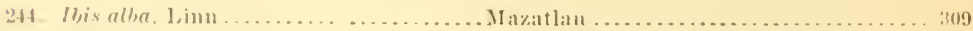

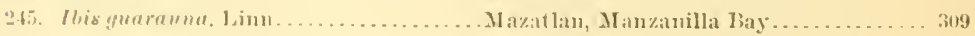

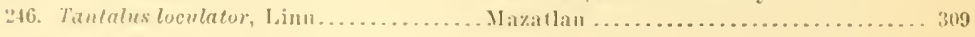

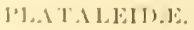

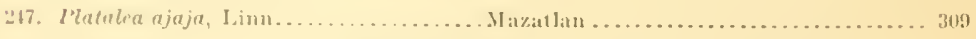

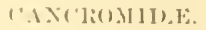

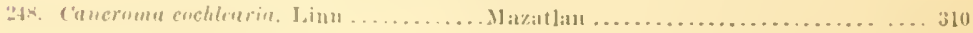


1874. LAWRECE, GEORGE N.-C'ontinued.

ARDEIHA.

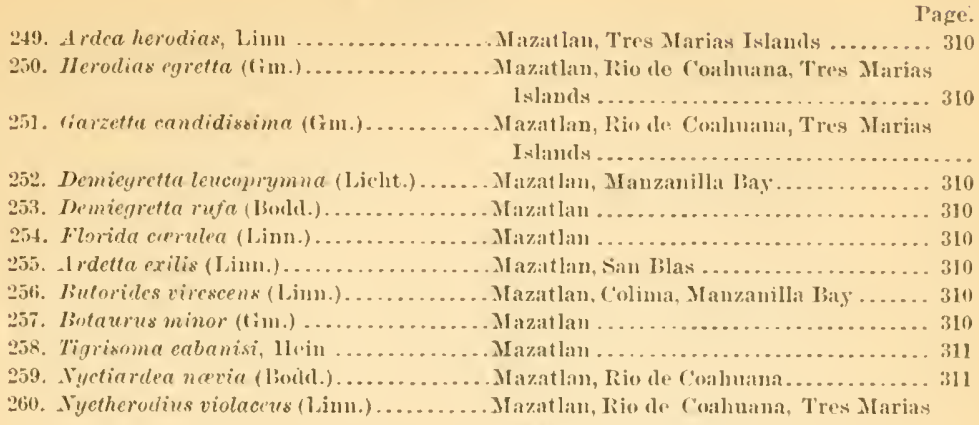

GRUIDA.

261. Cirus cunadensis, Limn

. Mazat lau

R.IILIII.F

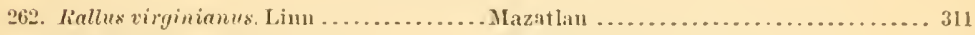

263. Lallus elegans, Aul................. Mazatlan ...................... 311

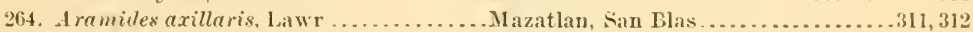

265. Porzana carolima (Linn.) . . . . . . . . Mazatlan ..................... 312

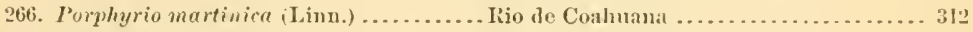

267. Gallinmla galeata, Licht................ Iazatlan, Tepie . . . . . . . . . . . ... 312

268. Fulice amerieand, Gm............... Colima. Mazatlan................ 312

\section{PALTIDEE}

269. Parra gymnostoma. Wagl.............. Iazatlan, Manzanilla Bay, Zacatula

Iiver. IRo dle Coahuana, Tepic ........ 312

\section{AX̃.TI1).E}

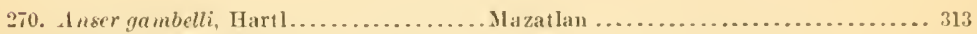

271. Berniela eanadensis var. occidentalis.

Baird......................... Durango....................... 313

s72 Dendrocyyna fulta (Gm.) ............... Yazatlan, San Ilas, Sonora, Sinaloa.... 313

2i3 Dendrocyyna utumnalis (Linn.) ....... Mayatlan . . . . . . . . . . . . . . . .313,314

$27 t$. nas boschas, Linu ................. Mazatlan ........................ 314

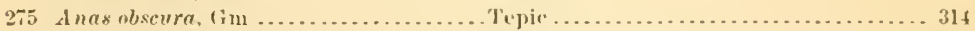

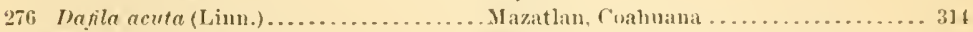

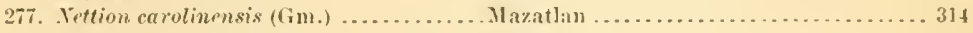

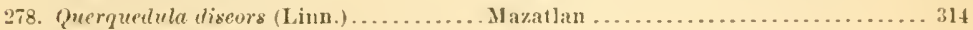

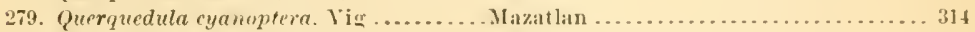

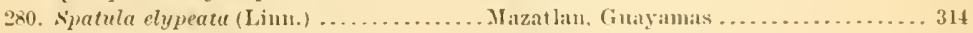

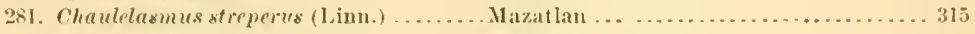

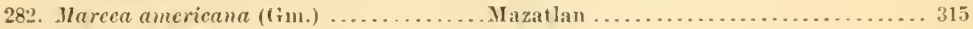

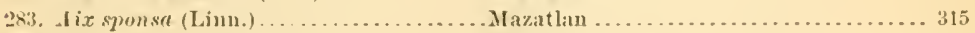

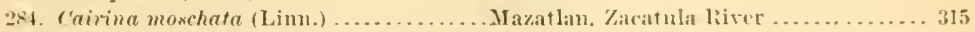

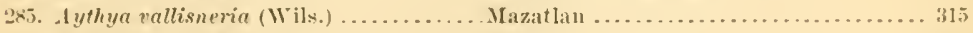

28ti. Iythya americana, Eyton ............ Mayat lan ....................... 315

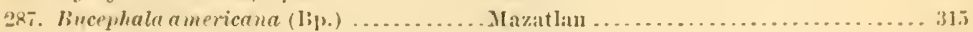

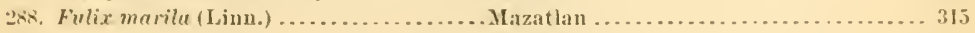

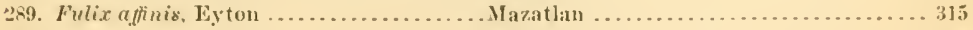

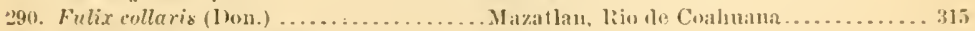

291. Erismatwa rubila (Wils.) . . . . . . . . . Mazat lan. Trpic . . . . . . . . . . . . 313

292. Erimatura dominiea (Linn.) . ....... Tepir ...................... 316

Bull. 40, N. MI.—-i) 


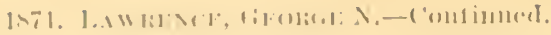

\section{l'lil.1:C.1.III.T.}

l'ilis:

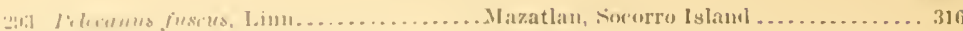

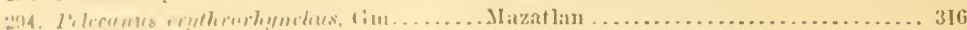

\section{SILLIII.E.}

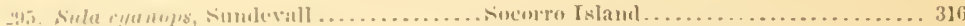

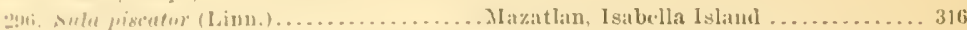

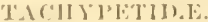

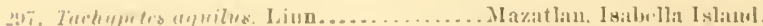

(iRATILIH)E.

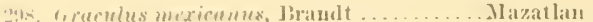

I'LOTIIIT:

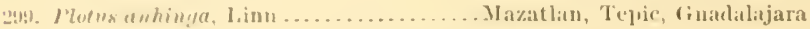

I'IIA L'TIIONII).E.

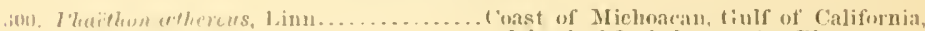

Islanel of Isibel, near Sin Blis.......317

L.ALIII.T:

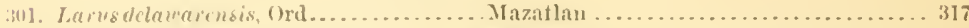

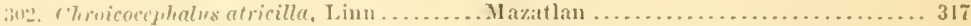

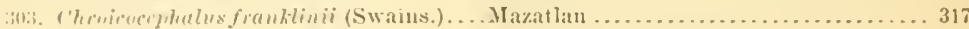

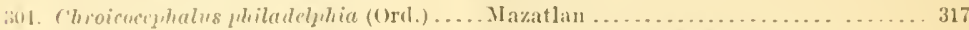

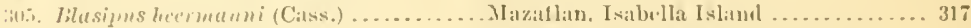

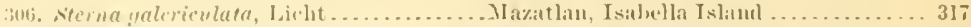

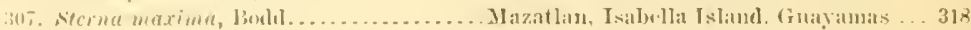

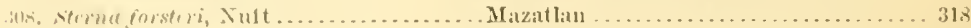

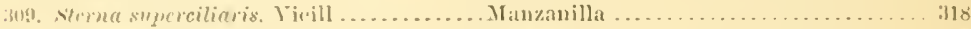

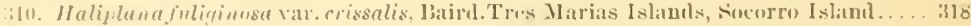

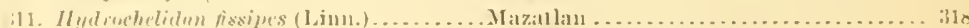

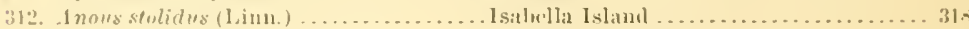

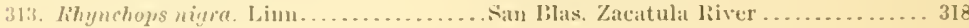

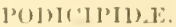

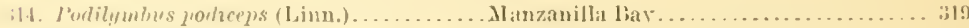

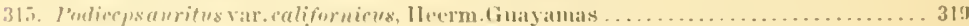

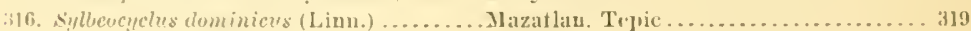

\section{7.}

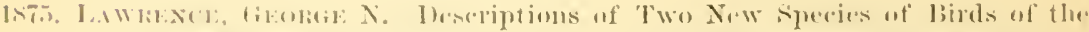

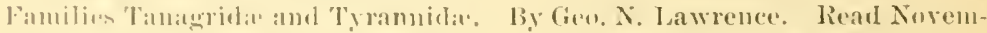

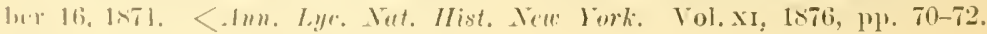
Isulat in Nis. 3, 1. Feluruary, 187.

Page.

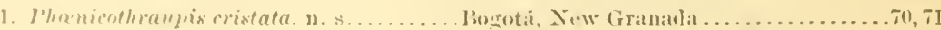

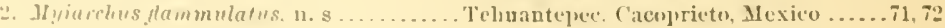

68.

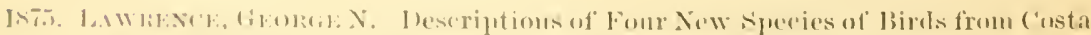

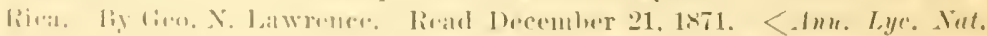

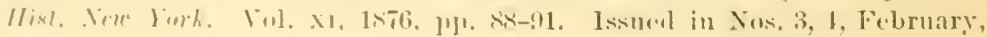
$1, \pi$ i.

I Cimmerifta arorutigule $11 . \mathrm{s}$

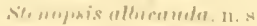

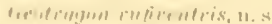

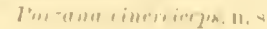

I'ate.

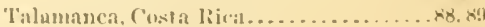

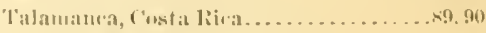

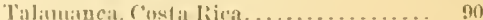

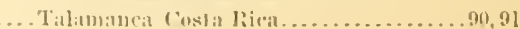


69.

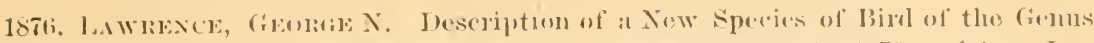
J'itangus, liy (ien. X. Lawrence, lieal Novemher 20), 1876. <.lun. L.yr. Tat. Hist, Iek Vork. Vol. x1, 1876, I'l. 288-290. Issucel in Xos.9, 10, 1) (ceulber, 187i;
1. Nitanyus yabbii, n. s
l'age. $2 n+290$

70.

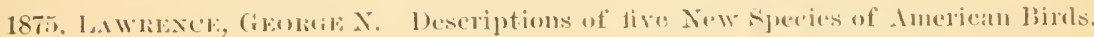

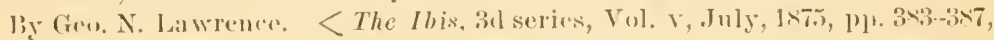
Plate No, Ix of Chlorospingus? speculiferus, Fig. 1, amel serpophuge leucura, rig. 2 .

Page.

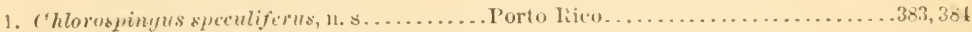

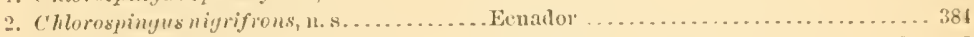

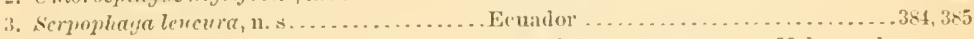

4. Orchilus atricapillus, n.s............... Costa lic'a, Ingostura, Volcum dlu

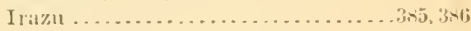

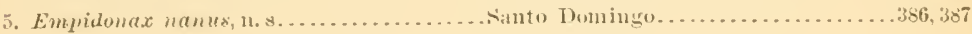

71.

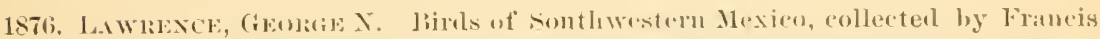

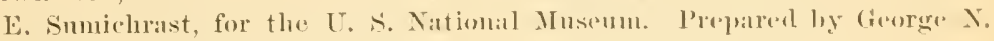

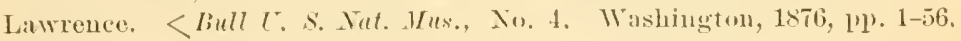
TVIRII).E.

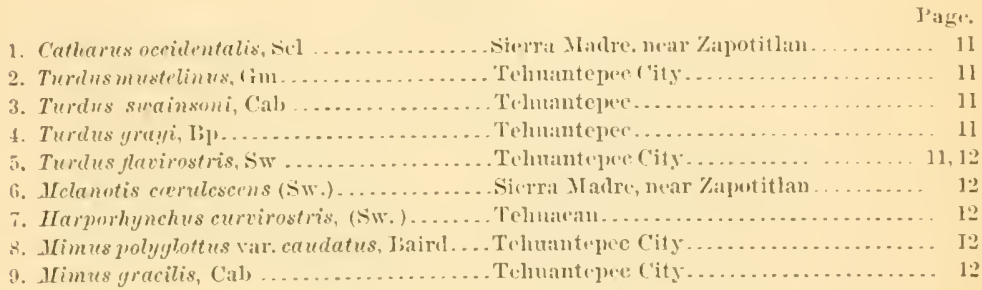

\section{SYLYIID.E.}

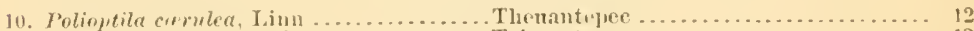

10. Polinutile albiloris,

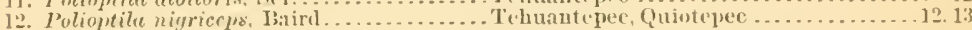

THORLOIYTTIIE.

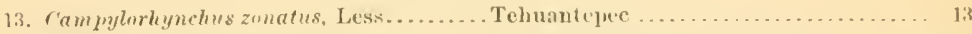

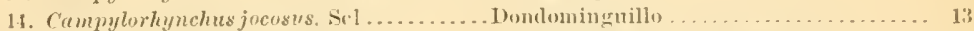

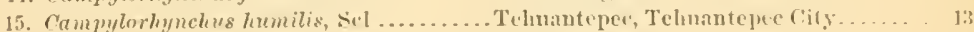

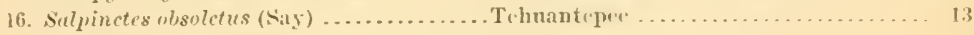

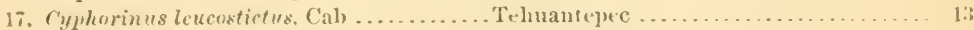

18. Theryothores maculipetus, Lafr ........ Tehnantepec .................. 1:?

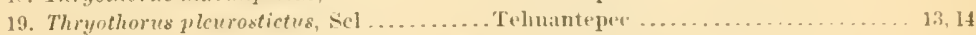

20. Thrywthorws bcuchic var. lucogaster.

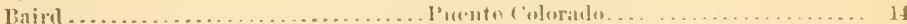

\section{MOTAILLII).F.}

21. Anthus lutoriciunux (1im.) Tehuanterue f'ity

\section{MNIOTILTID.E.}

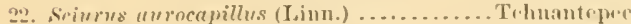

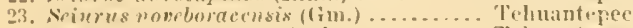

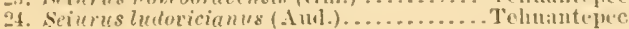




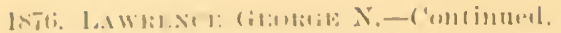

21i. I'urula "mmri enu (l,iun.)....

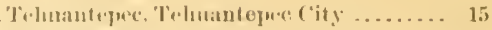

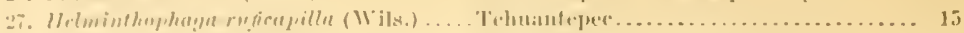

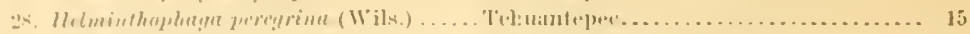

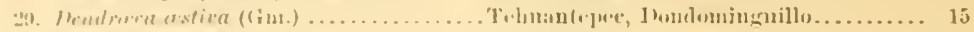

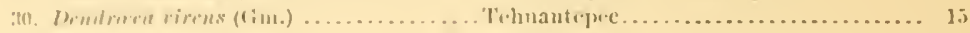

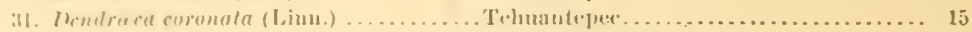

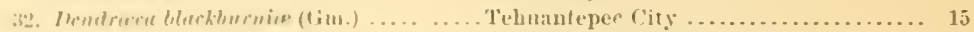

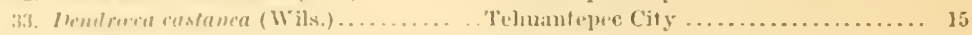

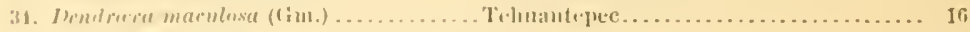

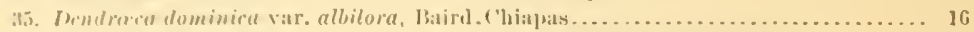

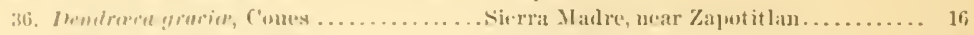

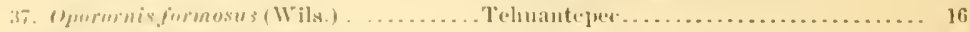

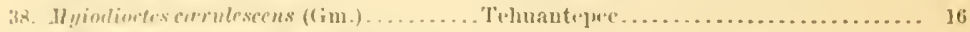

3!1. Myintiuctes pursillus var, pileolatws (P'al.

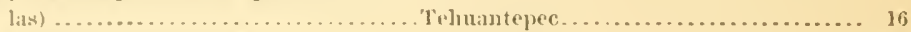

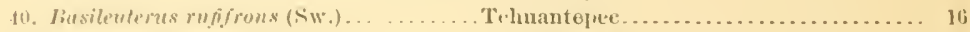

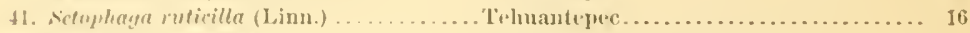

42. Nefol,

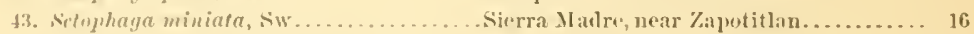

44. Granate'lus tenustus, J) Ihus........ Tehuantepec.................. 16

45. dimllhyis yiladetyiu var. macgilli-

rrayi (.Iut.) .................... Tohumteper, Tehuantepec City . ..... 17

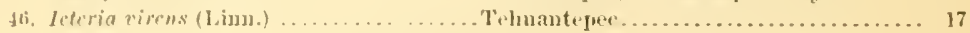

\section{IILUIMI11.T}

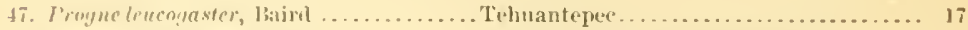

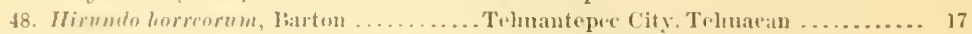

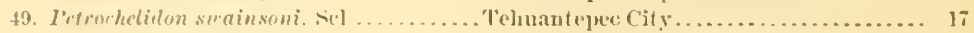

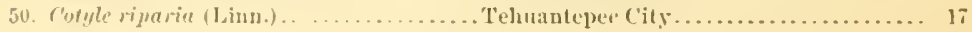

\section{VIIEONIII:}

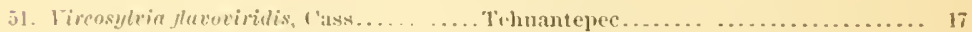

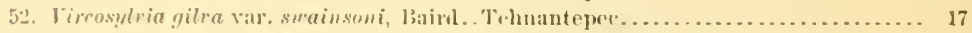

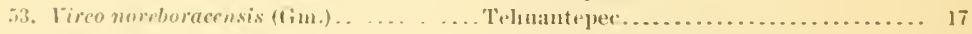

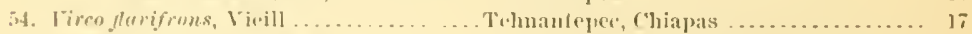

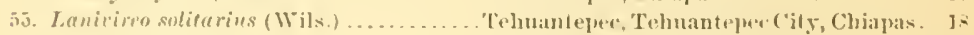

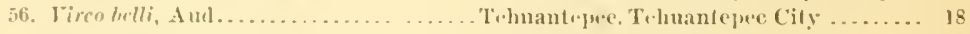

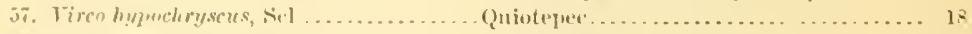

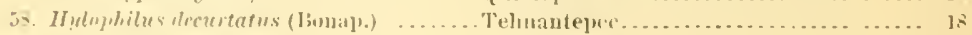

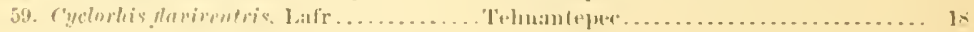

1.A.III).Y.

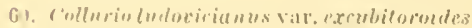

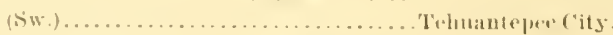

IIPELIIIS.

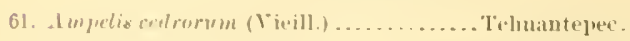

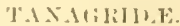

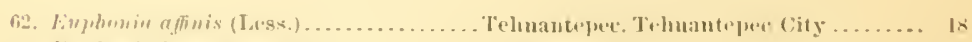

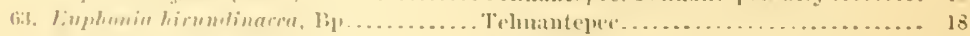

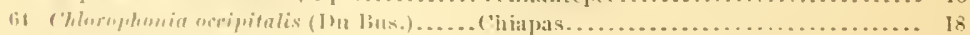

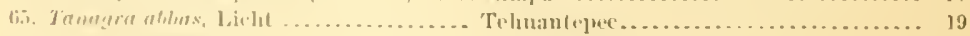

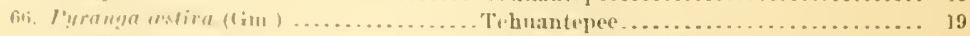

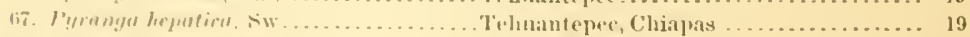

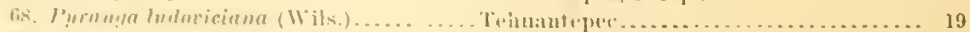

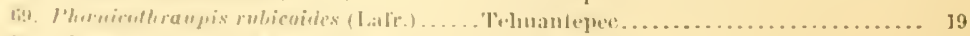

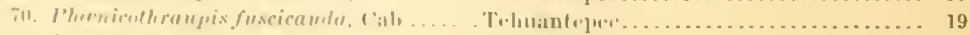

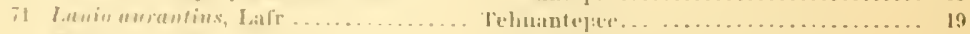

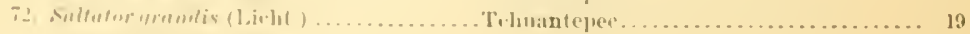

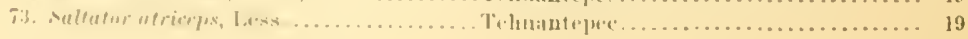




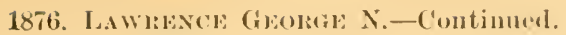

\section{FIINGILIII)}

l'age.

7t. Medymeles ludoricianvs (Linu.) ....... Trhusntepec.................. 19

75. C'urdinalis virginianus var. erermes,

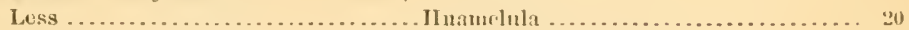

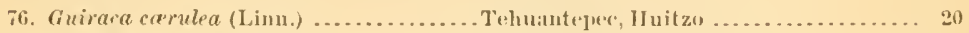

77. Guiraea parellina (Licht.) .............Thluantepee City. IIuallagn ........... 20

78. Vulatinia jacarina (Lim.) ........... Thusntepr................... 20

79. I'muipara pusilla (sw.) ............. bundominguillo .................. 20

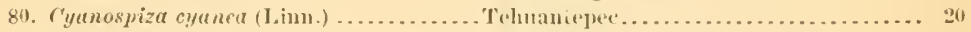

81. ('yenospiza ciris (Linn.) ............... Thelunnteper, Tehuantelec ('ity ........ 20)

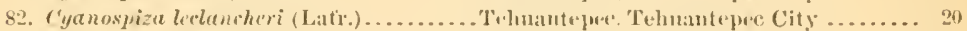

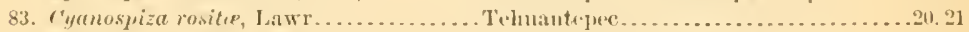

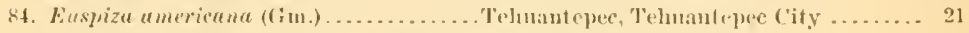

85. I'ussereulus savanna var, aluelinus, 13p. Trohuntrpec City.................. 21

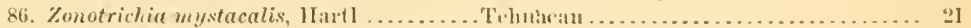

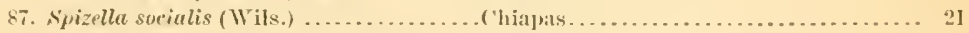

88. Spizella socialis var, arizona, ('mes..... Thnumtepec ................... 21

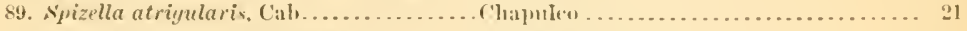

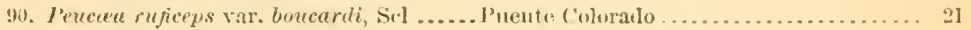

91. Coturniculus passerinus (Wils.) . . . . . . Tehuantepec City . . . . . . . . . . . . 21

92. Embernagra rujirirguta, Lawr . .......11 namelula ................... 2.

93. Hamophila rufescens, Sw ............ Tuluantepec................... 22

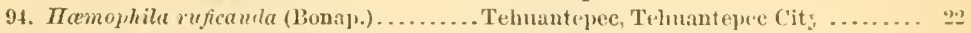

95. Hamophile sumichrasti, Lawr......... Tchnantejec.................... 22

96. Pipilo maculatus, $\mathrm{sw}$............... ('icneguilla .................. 22

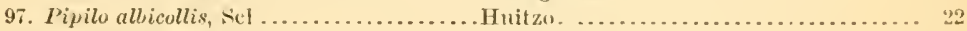

98. Chondestes grammaca (sily) ........... Th huatepec................... 22

99. Chrysomitris notata (Du liss.) ......... Chiapals..................... 2 .2

AI.ATIIIT:

100. Eremophila alpestris rar. chrysolema

(IVagl.) .........................'Thlunteper .

\section{ICTEIIDE.}

101. C'assiculus melanicterus (Bp.)..........Tehmantepec. Tehuantepec Cit $5 . . . . . .23$

102. Cassicus prevosti (Less.) ............ Trhunteprec.................. 23

103. Ieterus prosthemelas (Strickland) ...... Telunantepec.................... 23,

104. Icterus melanocephalus (Wagl.) ........ Tehuantepec.................... 23

105. Icterus mesomelas (Wagl.) ........... Tehuantepee................... 23

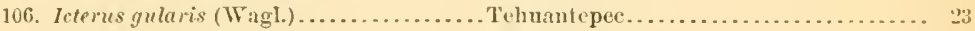

107. Iterus pectoralis (Wagl.) . ..........Thuantepec.................... 23

108. Ieterus formosus, Lawr..............Tehnantepec.................. 23

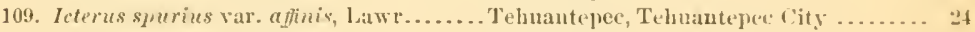

110. Molothrus ameus (Wigl.) ............. Thuantepec.................. 24

111. Jolothrus pecoris var. obseurus (1im.)...Trhantepre City................. 24

112. Stumella magna vit. mexicana, kil. ... Tehnanteper.................... 21

113 Quisculus macrurus, Sw ............. Thehantepec.................. 2k

114. Quiscales mexicanus, Cass ........... Tehuatepec................... 21

\section{CORTID.l:}

115. Cyanacitta coronata (Sw.) ............ Cieneguilliı ................... 24

116. ('yanocitta califormica var. sumichrasti.

livlg......................... vacaltepec....................... 24

117. Calucittu formosa (Sw.) .............. Telunanteper, Telunantepe ('ity .......24, 25

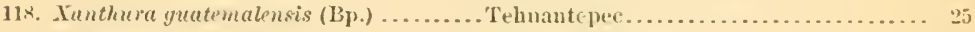

119. I'silorhinus morio (Licht.) ............ Tehuaut pec.................... 25

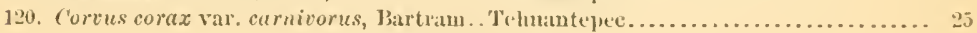

1)ENHIOCOL.AP'JID.F.

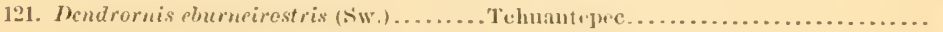




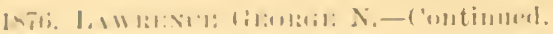

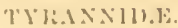

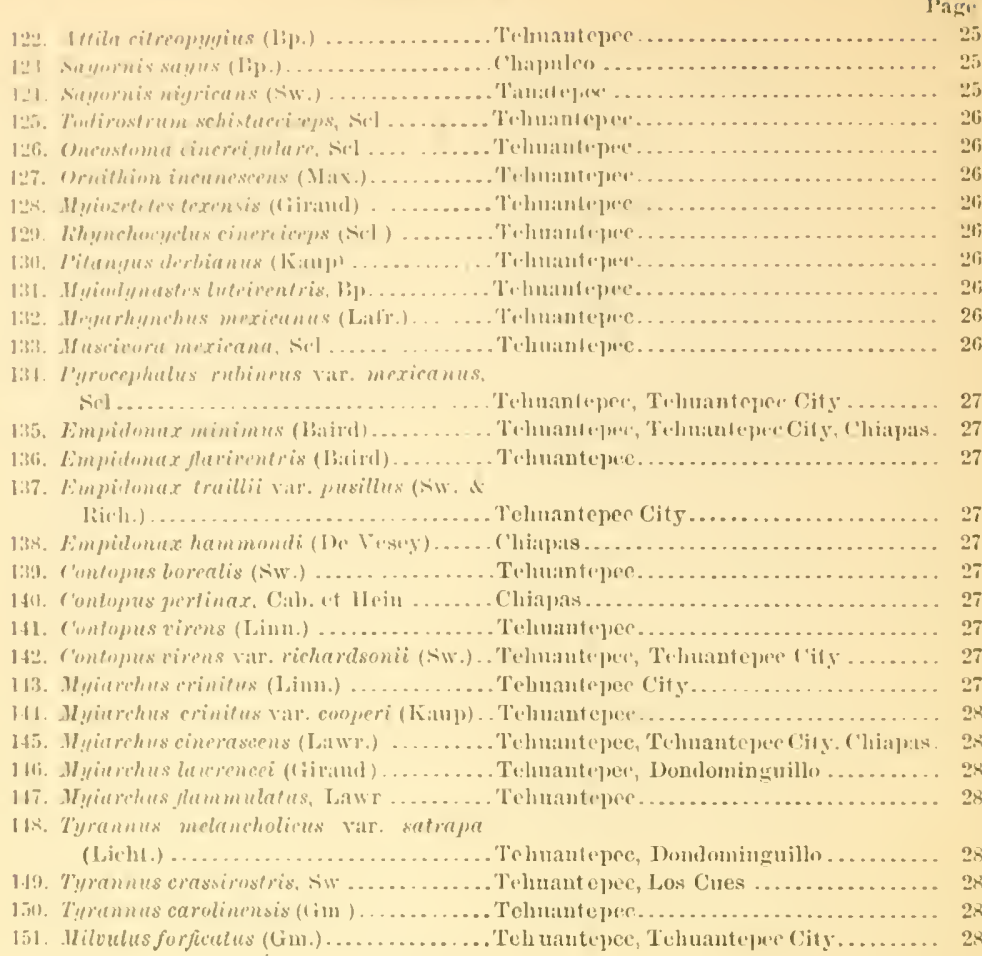

\section{(1)Tixtillet.}

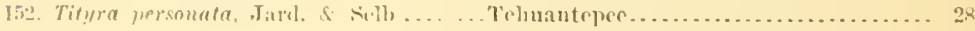

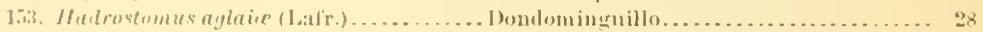

1'I1'IIII.

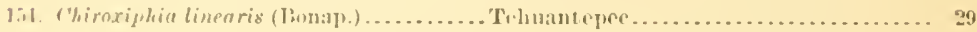

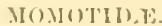

15i. Momotus lessomi, Less................ Tehnantrpec................... 29

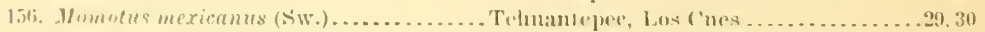

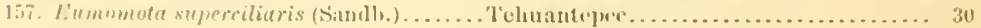

\section{A LCWIIXIDE.}

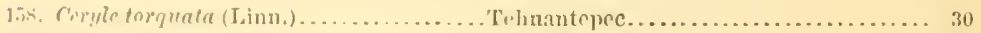

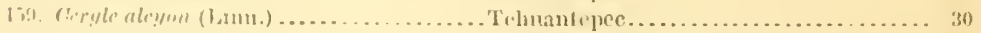

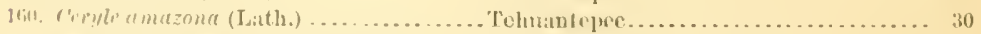

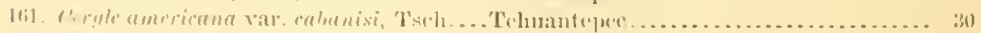

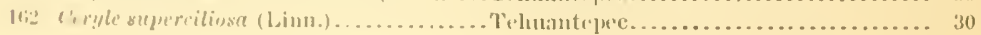

1:(19.1) 111$) .1:$

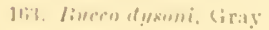

Tehnamlepers.

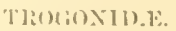

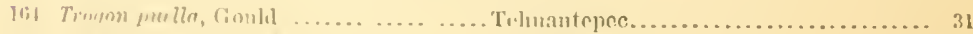

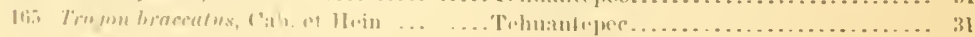

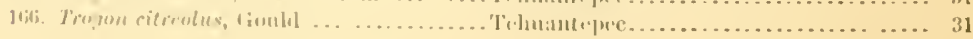




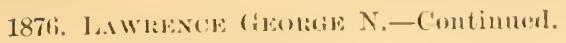

CAPRIMULIDA.

I'age.

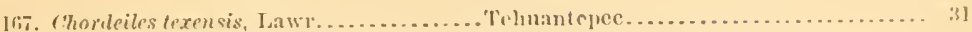

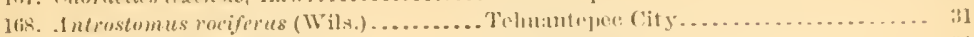

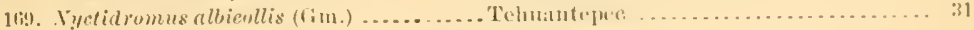

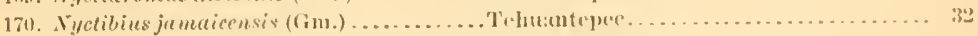

('VIPSELIH).

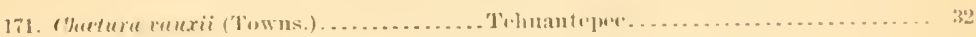

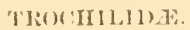

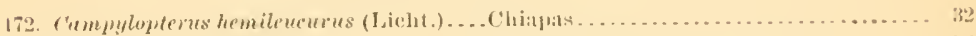

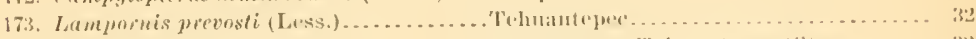

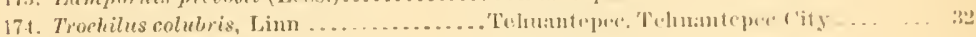

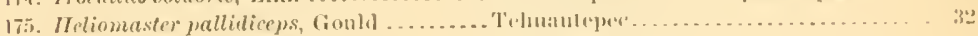

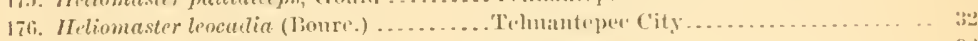

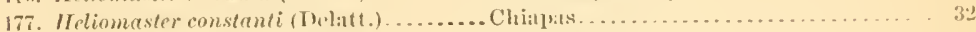

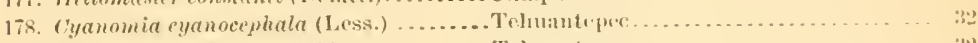

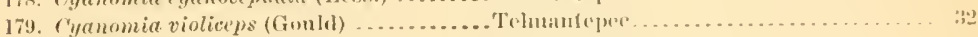

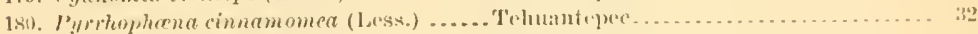

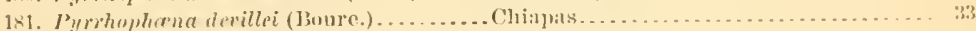

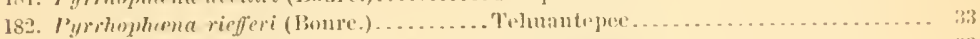

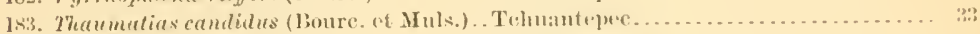

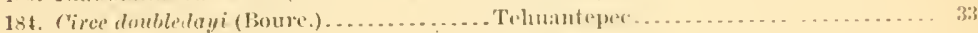

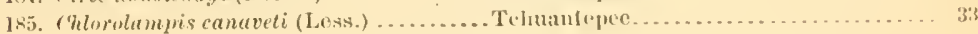

\section{CIT'LLIIIE.}

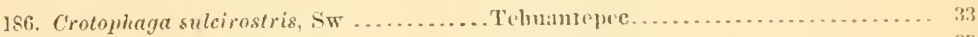

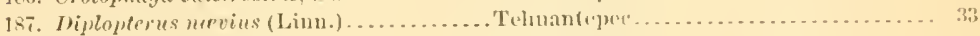

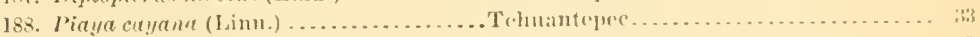

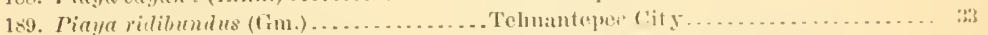

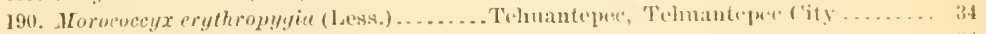

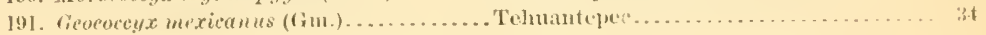

\section{I:AMIIIASTIIXE.}

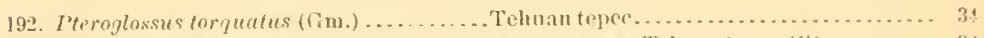

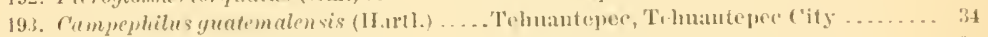

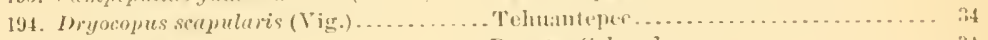

195. P'icus sealaris, Wagl ................. Puente ('olurado . .................. 31

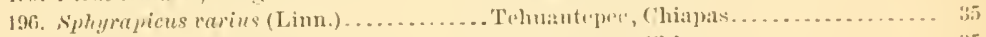

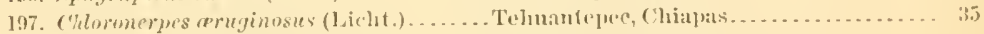

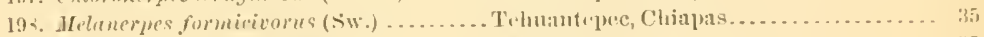

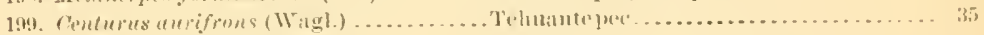

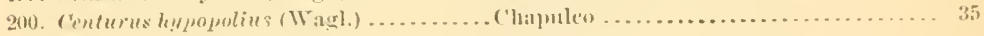

I'SITTACIUT.

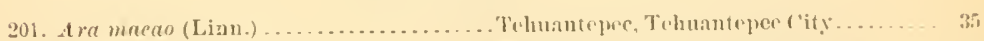

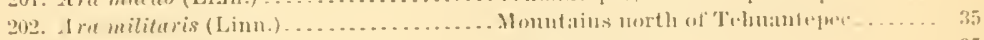

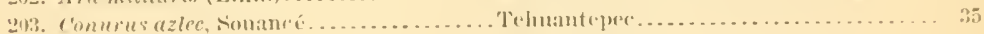

204. ('murus petzii (Leih.) ............. Tehuintrpe.................. 36

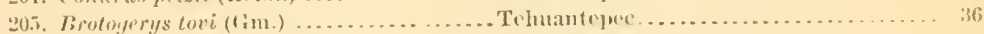

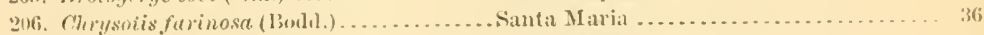

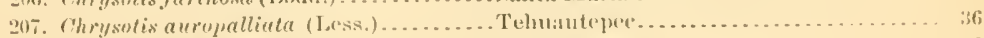

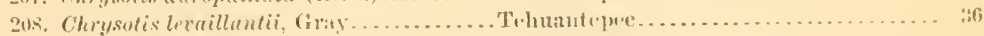

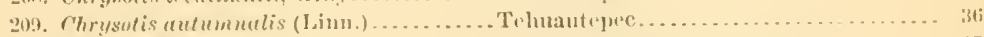

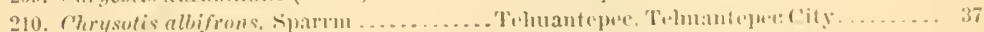

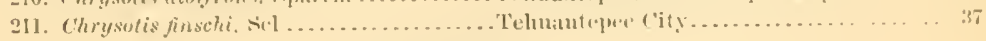

\section{STlil(il).T.}

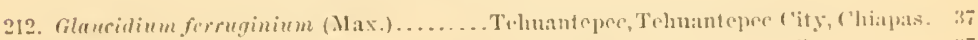

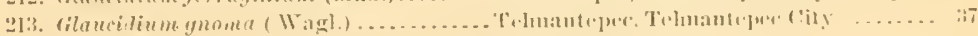

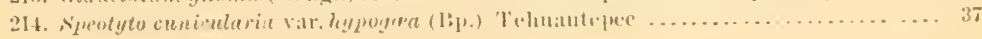




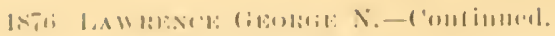

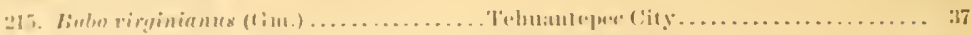

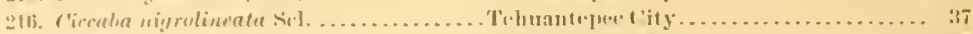

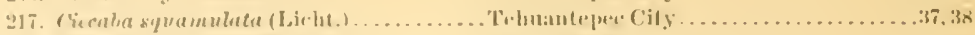

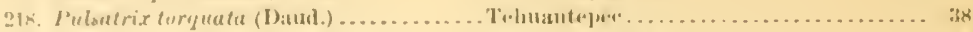

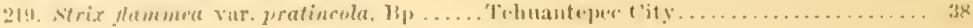

FALA'(ONII).

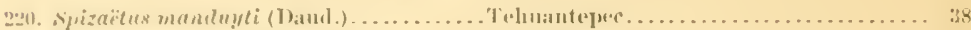

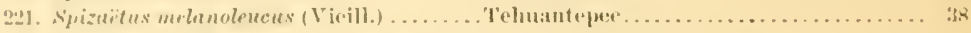

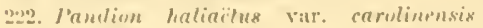

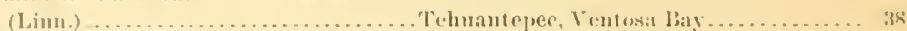

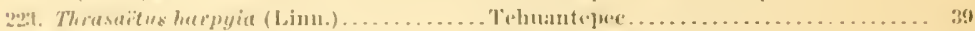

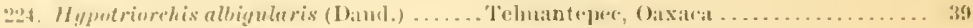

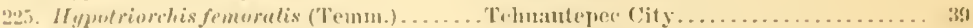

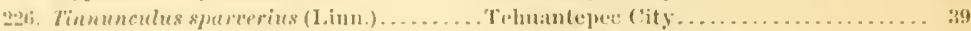

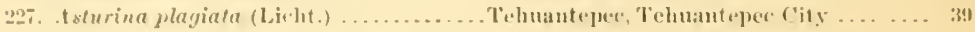

225 . Lupormis maynirostris var. grisevaula,

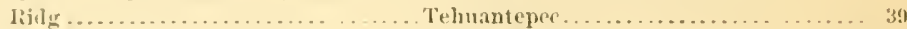

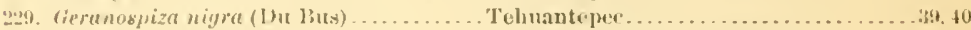

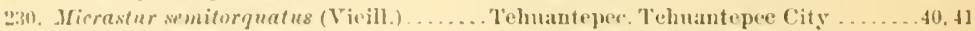

331. Werpetolleres cuchinuths (Linn.)....... Tehuantepes, Tehuantepee City ...... 41

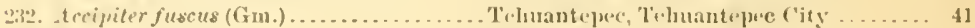

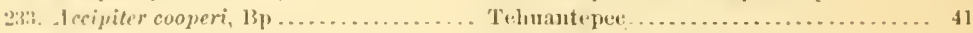

2:34. Suteo borealis (Gm.) ................. T'huntepe City. C:acopricto......... 4)

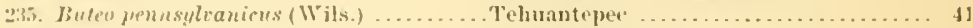

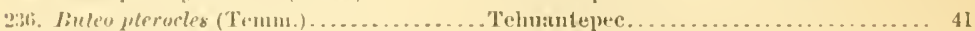

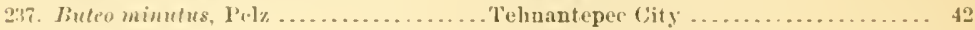

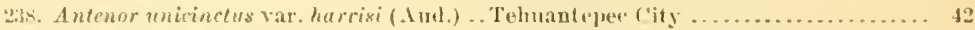

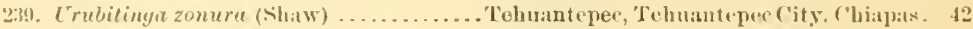

240. Trubitinga anth racinu (Licht.) ........ Telututepre................. 42

241. Cymindis cuyenensis (tim.) .......... Tuhuntepec..................42. 4:

242. Cymindis uneinatus (111.) ........... Trhututepee................. 43

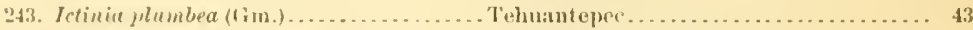

244. Circus cyaneus var. hudsomius (! inu.) .. T'ehnantepet................. 43

245. Polyborus thates vir. andubonii. C'ass... Istlunts of Tehuantepe.e........... 43

IATIIAIR'ID.F:

246. Cathartes papa (I.im.) . . . . . . . . . . . Ist limus of Tehmanteper ........... 43

247. Rhynoyruphus uuru (1.inu.) ........... Ist hmus of Tehutufupe ............ 44

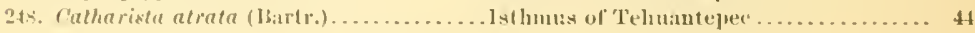

\section{CULТМIII):.}

249. Columba favirostris, Wagl .......... Teluantepee.................. 44

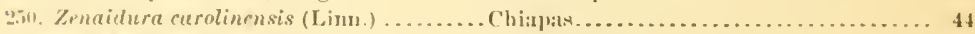

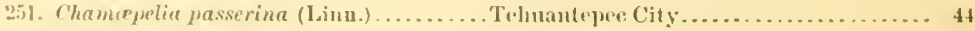

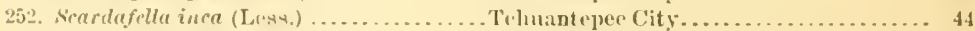

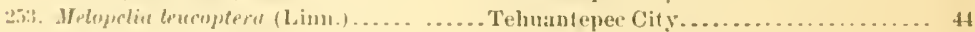

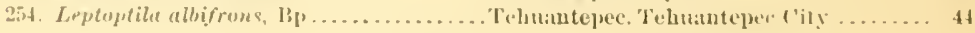

C'RiA ('I1).F

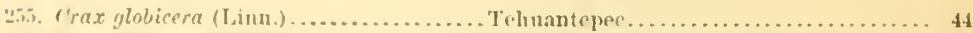

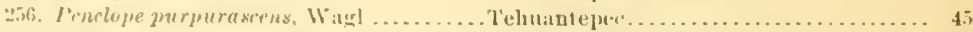

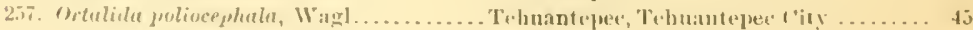

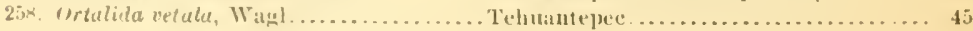

PEIIII'IIt.F.

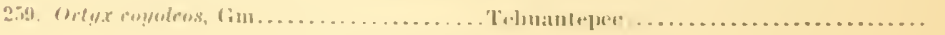

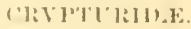

2601. Ninthescerrus sullisi, liy

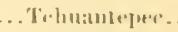


ITIBISHEI, WRITINGS OF GEOR(AL NEWHOIA IAWRENCE. 73

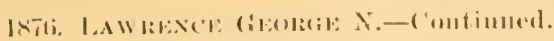

(IIAIIIRIIIS.

I'ige.

261. Edienemus bistriatus, Wigl .......... Tehunntepec.................. 45

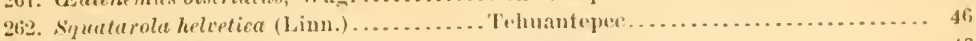

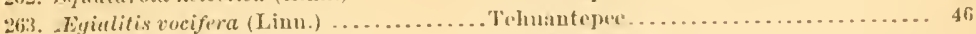

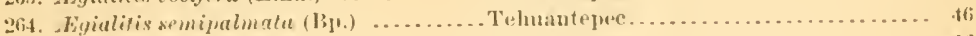

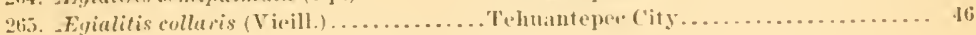

\section{II.E.IATOPIINA}

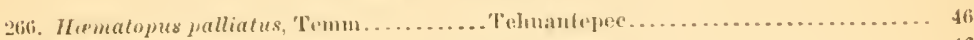

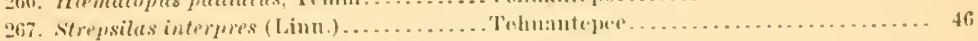

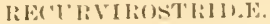

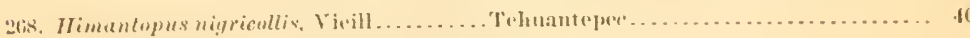

\section{I'IIL.IIRIII.E.}

269. Lobipes hyperboreus (Link.)

I'vhunutrpe.

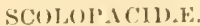

270. Mucrorhamphus grisens var. seatopaceus

(siv) ....................... Tehuanteper.................. 40

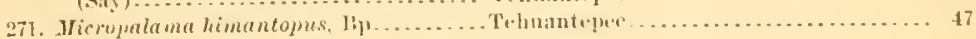

272. Erewnetes pusillus var. oceilentuliv,

Lawr......................... Tehnantepec, Tehuantepe ('ity ....... 47

273. Letodromas minutilla (Virill.) ......... Tehnantejec City................. 47

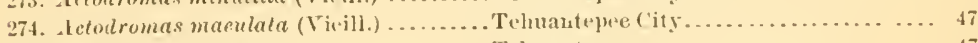

275. Calitris arenaria (Linu.) ........... Trhuantepe.................... 47

276. Limosa fedoa (Linn.) ................ Teluantepee.................. 47

27. Symphemia semipalmata $(\mathrm{im}$.) . . . . . Tehmantepwe.................. 47

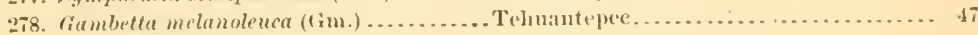

279. Gambetu flavipes (Gm.) ............. Tehuantepre.................. 4i

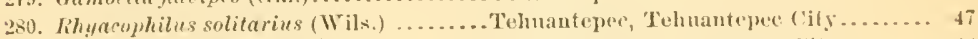

281. Tringoiles macularius (Limn.) ......... Telnanteper, Tehmantepe ('ity........ 4.

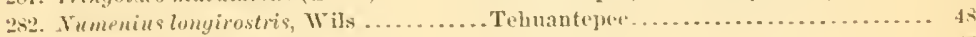

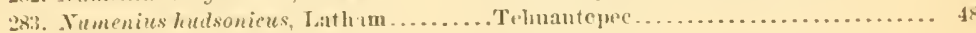

\section{T.INTAIID.L.}

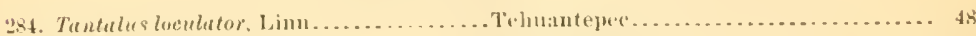

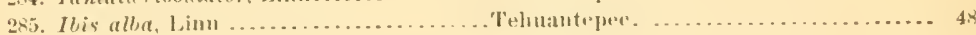

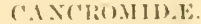

236. Cancromu corlentia. Liun .......... Tehnanteper.................. 4.

\section{I'I.ITALEII.F.}

2x7. Mlatulea ajuja, Linn

Trhuntepere.

\section{ARERIIR.F.}

288. Arlea herodius, Liun ................ Tehuanteper Ciry . ............... 4.

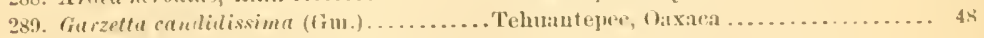

220. Demiegretta leucugastra vit?. leneo-

prymna (lieht.) .................. Tehtante pree City................. $4 s^{\prime}$

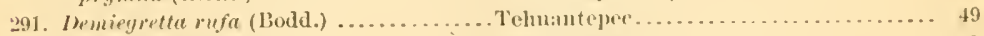

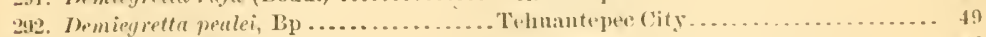

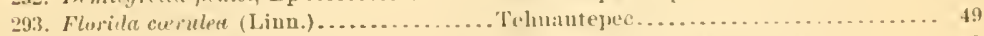

294. Buteriles vireseens (I.inn.) ............T. Thuantepec. Tehuantepe City ....... 49

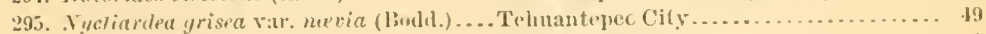

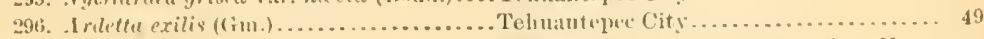

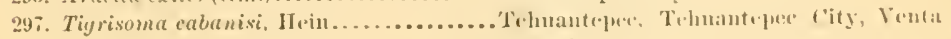

siılitl: ....................... 49 


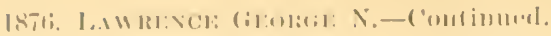

\section{1:.11,1.111.1:}

J'igne.

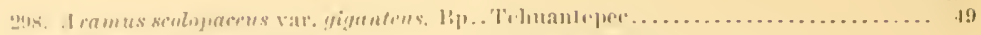

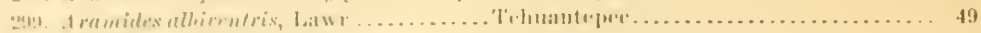

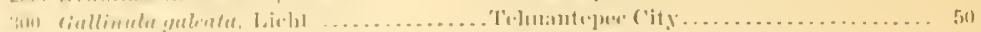

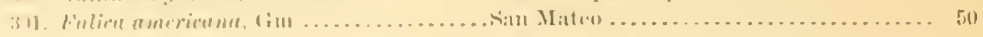

\section{I'. IRI:I11.1:}

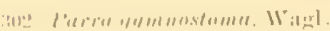

Tr.huantı.

50

\section{INAIII.1:}

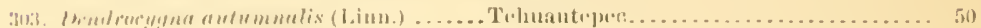

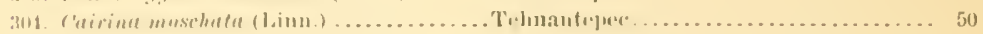

\section{I'LLEL:AN11E}

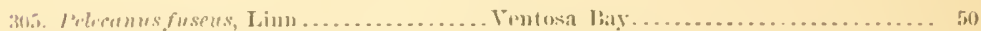

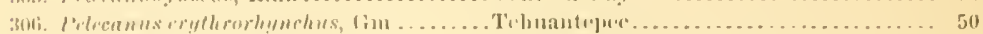

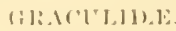

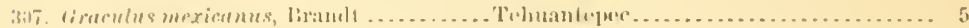

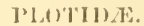

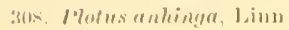
.'T'linanteprer.

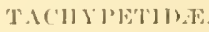

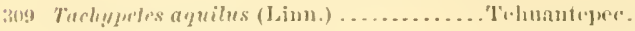

\section{LAIII) E.}

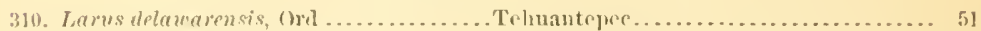

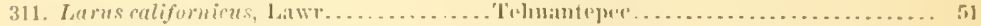

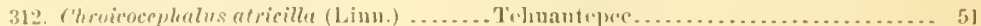

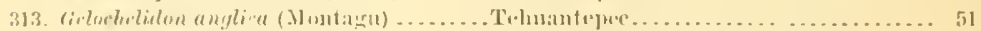

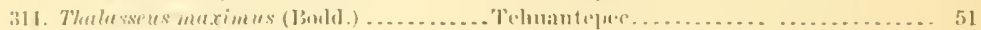

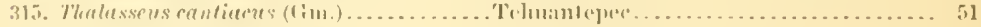

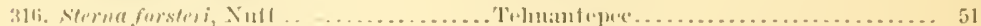

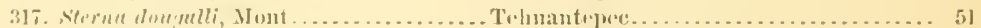

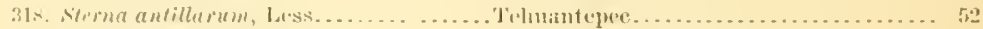

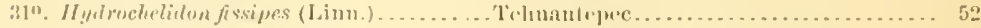

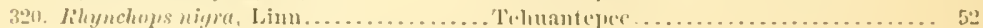

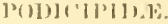

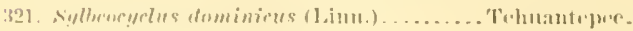

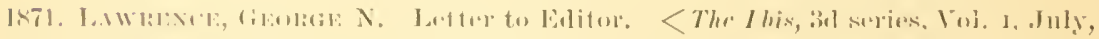
$1 \times 71,1.370$.

Prige.

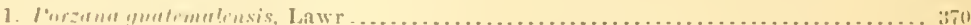

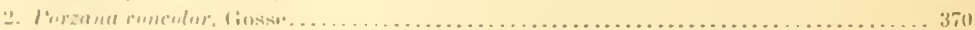

73.

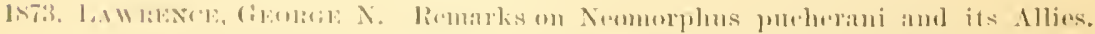

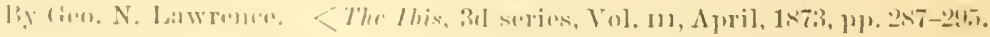

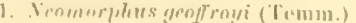

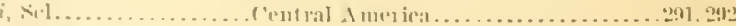

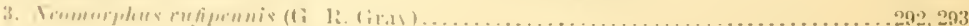

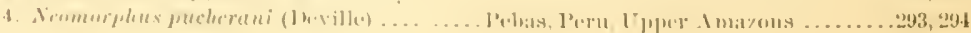


74.

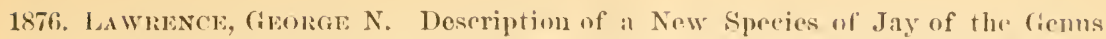
(yamocitta; also of a snpposed New speries of the Groms Cyanoworax. By

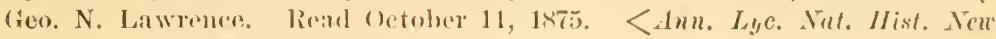
limk. Vol. xI, 1×iti. 1?1. 1683-166.

t.

1. C'yanocittu pulthra, n. s................ (quito. Ecualor.................... 16

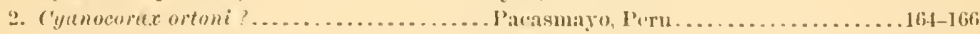

75.

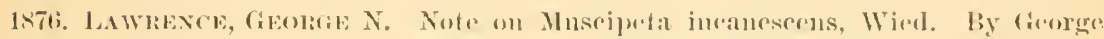

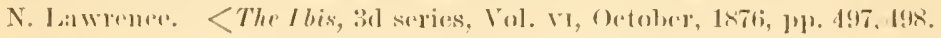

1. Muscipeta incraescens, Wircl 497.498

76.

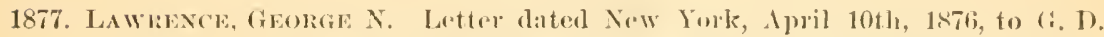

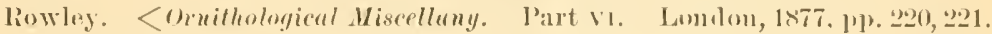

I'ilgit'.

1. Somateria labratoria (J. F. (Mmelin)

$.20,201$

\section{7.}

1876. Lawhexce, George N. Oceurrener of the bamacle (Goose (Bernicla leneopisis) on Long lsiand, N. Y. liy Geo. N. Lawrence. <liull. Nutl. Truith. C'lub. Vol. 11, No. 1, Jamury, 1877, p. 18.

1. Ternich leucopsis, Boin

Page. Long Islant, N. I 18

\section{8.}

1877. Lawriace, George N. A Note on Cupidonia enpido var. pallidionetus,

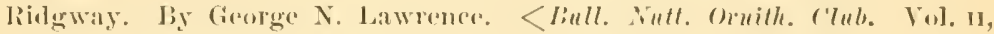
No, 2, April, 1877, 1. 52.

Piage.

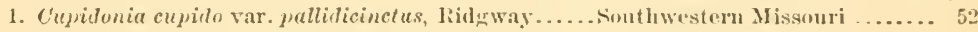

79.

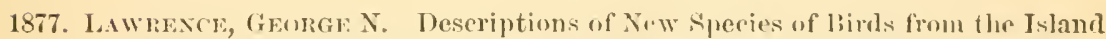

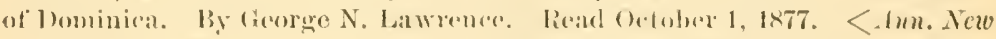
Fork Acul. of s'ci. Vol. 1, 187!, 111. 16-19. Issmed in Nos. 1, 2, Jee., 1877.

1. Thryotherus rufescens, it s

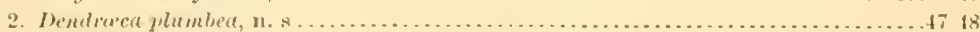

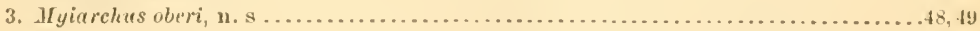

80.

1877. Lawnexce, Geonge $\mathrm{N}$. Deseriptions of New Speeies of Birds of the Families

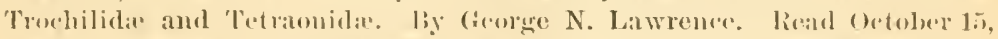

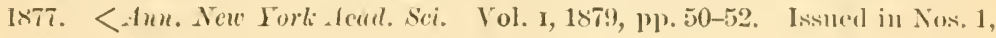
2 , 1)er., 1877.

1. Sirolaliuus liage.

New Prorieltrece, IV. I ............... 5u

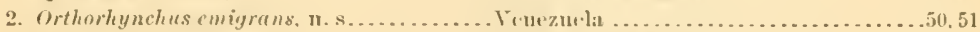

3. Cyrtonyx sumichresti, n. s.............. Sint: Etigenia, Hexico ?...........51, 52 


\section{1.}

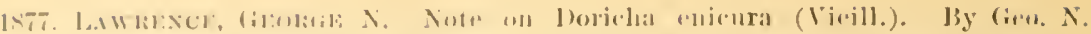

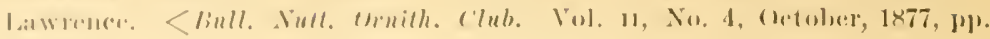
(1) $1,10 ! 4$

1. (ilutherax lacifer (Niw.) is

l'age.

Arizinin.

108,109

82.

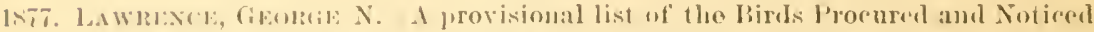

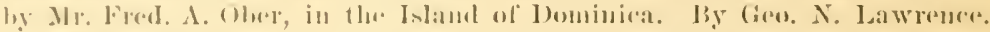

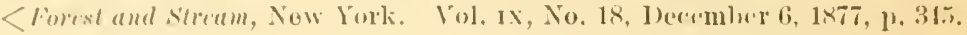

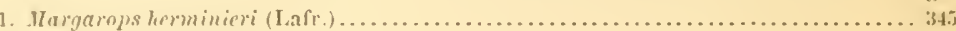

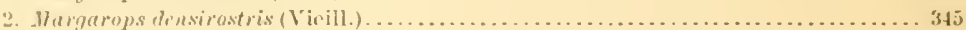

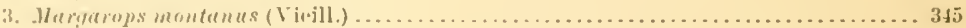

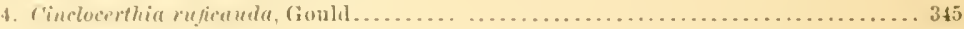

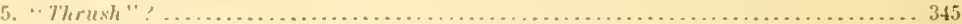

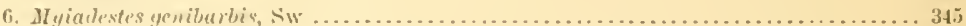

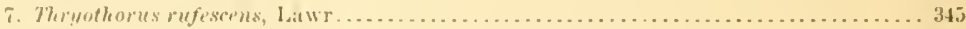

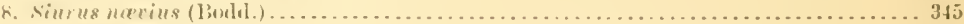

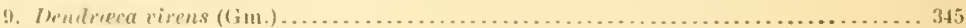

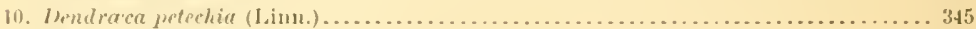

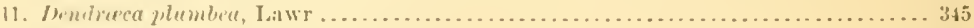

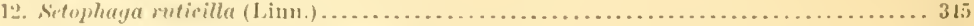

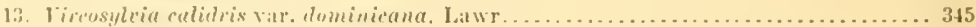

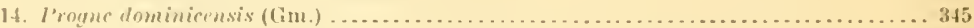

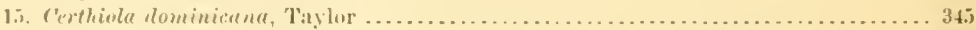

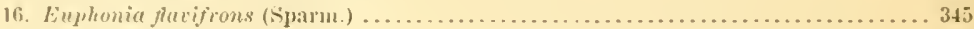

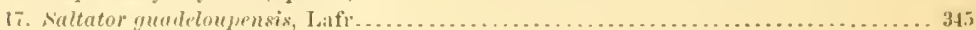

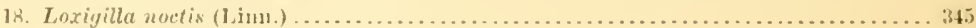

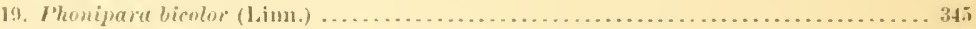

21. Elainea martinira (Linn.) . . . . . . . . . . . . . . . . . . . . . . . . . . 345

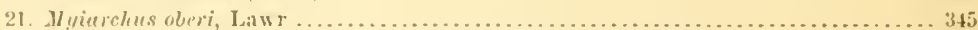

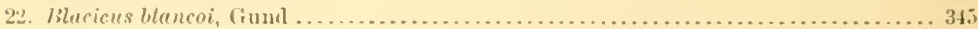

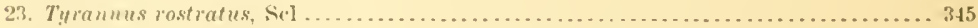

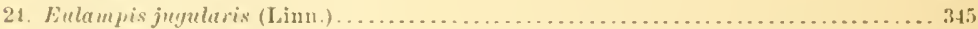

25. Eulampis holosericeus (Lim.) . . . . . . . . . . . . . . . . . . . . . . . . . 345

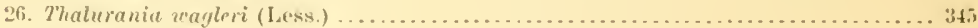

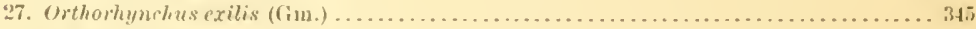

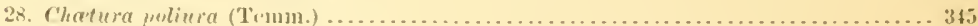

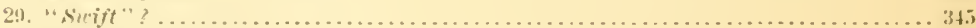

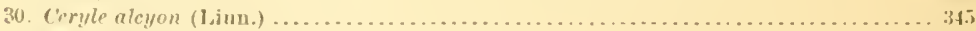

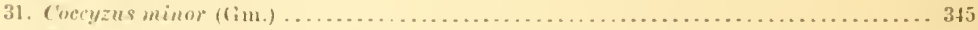

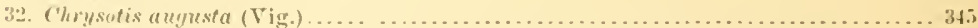

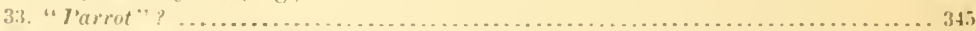

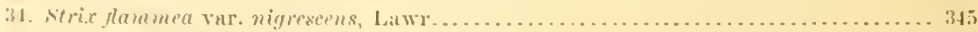

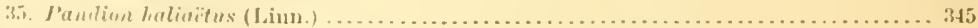

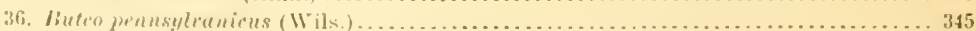

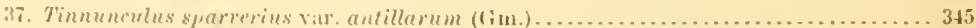

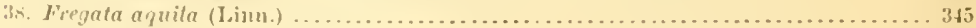

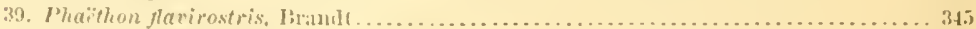

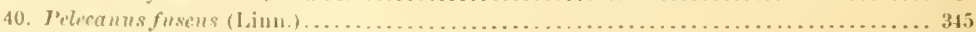

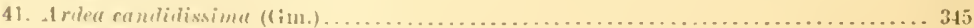

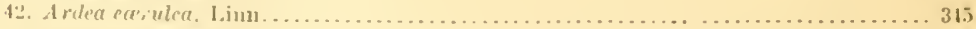

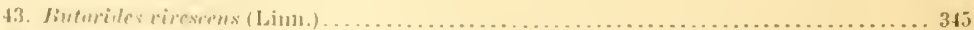

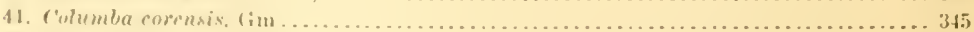

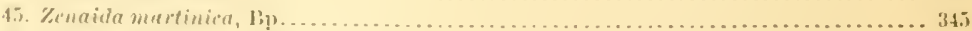

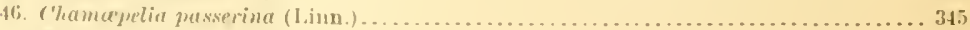

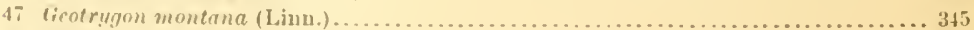

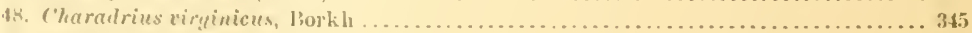

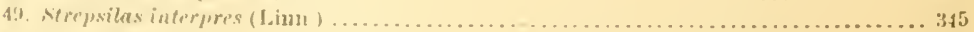

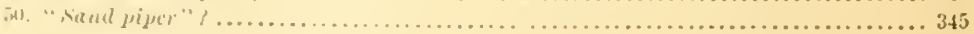




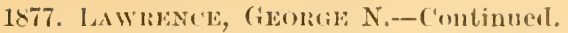

l'age.

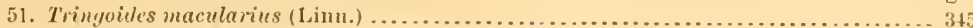

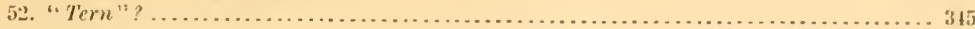

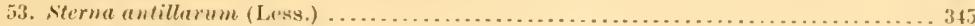

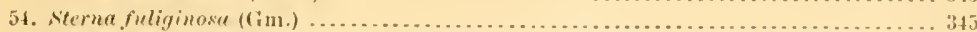

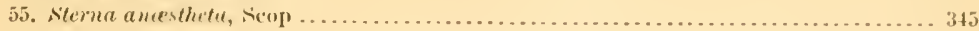

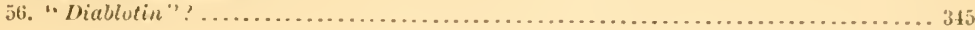

83.

1878. Lawrexce, Georit: N. Characters of a suppused new species of south American Thrush. lig (ieo. X. Lawrence. <The Ihis, the series, Vul. II, January, 187s, 1. 57, l'late No. 1.

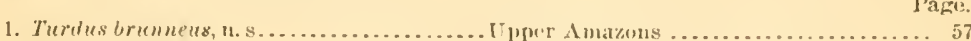

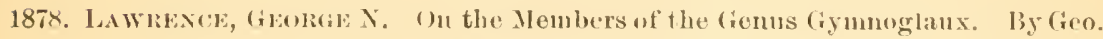
N. Lawrence. <The Ibis, Ith serios, No. 1;, Vol. 11, April, 1878, pl. 181-187.

1. (i) I’agu:

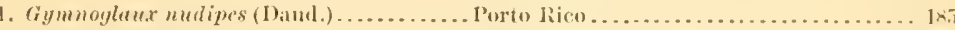

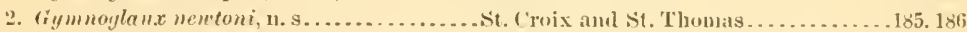

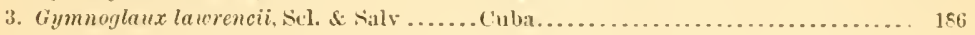

85.

1878. Lawnexce, Georate N. Deseription of a New sperias of parot of the fienus ('hrysutis. By George N. Lawrence. lieal Mareh 26, 1878, <.lnn. Yew Fork Acad. Sci. Vol. 1,1879, 11, 125, 126. Issued in Nos. 3, 1, . pril, 1878.

Page.

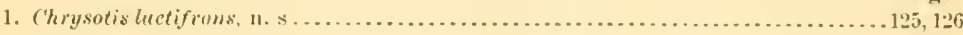

86.

1878. Liw Island of St. Vincent, Wast Indies. By George N. Lawrence. Read May 13th, 187x. <inn. New Ferk Acad. Sci. Vol, 1, 1879, pp. 146-152. Issued in Nos. 5, 6, May-September, $18 \pi x$.

1. Turtus nigrirostris, n. s................St. Vincent, $W .1 \ldots \ldots \ldots \ldots \ldots \ldots \ldots . .146,147$

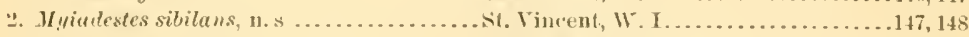

3. Thryothorus musicus, n. s................t. Vineent, W. I . . . . . . . . . . . 148, 149

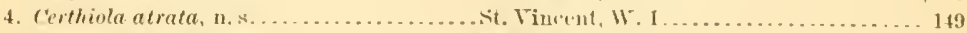

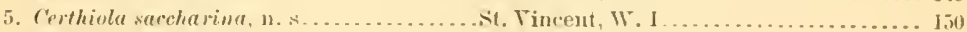

6. Leucopteza bishopi, n. s . . . . . . . . . . . . . . Vincent. W. 1 . . . . . . . . . . . . 150, 151

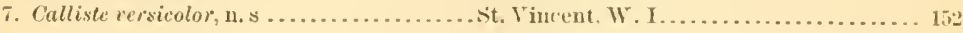

87.

1878. Lawhesce, (iEorat: N. Descriptims of supposed New spentes of Birels from

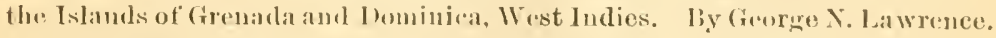
Real June 3d, 1878. <Amu. Yex Fork Icud. Sci. Vul. 1, 1879. 1p. 160-16i3.

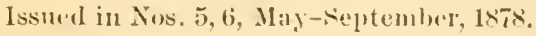

1. Turdus caribberus, 11.8 Page.

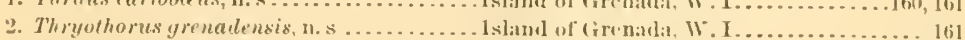

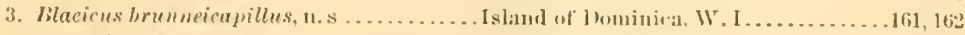

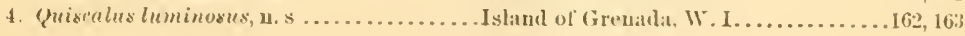




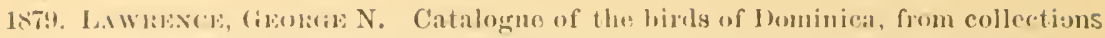
mad, for the smithsomian Institulion, by Eresleriek 1. Oher, together with

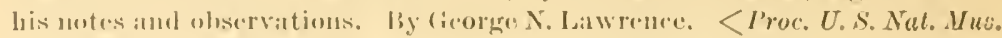
Vul. 1, IsT', Pl. I8-69.

TILIIII.

1'ago.

1. Maryurnzs herminieri (Lafr.).

2. Jlengurops densirestris (Tivill.)

3. Jlaryarejs momtamus (V'iesll.)

1. C'inclocerthia reficalu, fiould.

5. Thrush?

SYLVIII) E.

1i. Myiudestes geniburbis, sw

'TIEO(IL(II) Y'TII).F.

7. Thryothorus rufescens, Lawr.

SYLVICOLIDE.

8. Siurus necius (Indil.)

9. Dentrece virens (Gim.)

10. Itendraed petechia (linn.)

11. Dendreace plumbere. Latw

12. Setophaya ruticilla (Linn.)

VIREONIDE.

13. Viresylvie culdris var, dominieanu, n, s

\section{IIIUXIMIII.E}

14. I'rogne deminicensis (Giul.)

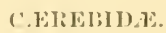

15. Certhiole deminicuna, Taytor'

TANAGTII)

If. Euphenia Jlavifrous (-.'parm.) 56 . in

17 saltator yuadelonuensis, Latr

FlIITHLLIDE.

IA. Loxigilla noctis (l,iun.)

19. Ihonipara bicolor (Linn.)

TYRANXIIE.

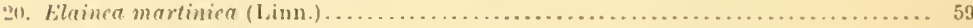

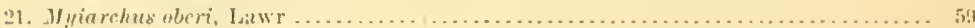

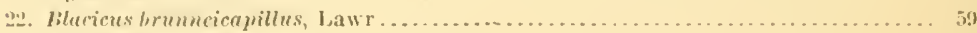

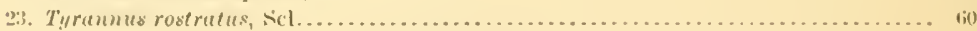

Tli()(III,I]). 1:

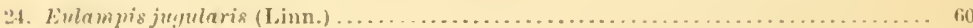

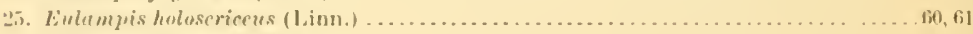

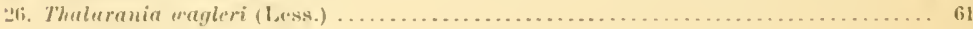

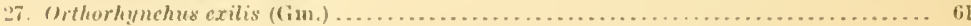

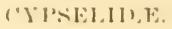

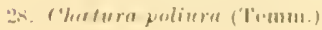

$2 ! 3$. swifl?. 


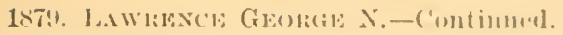

\section{IITEIINIIIS.}

30. Ciryle ulewon (linu.)

IIITLII I.X.

31. ('ocogzus mimor (fill.)

IPIT T.MPIIT:

32. ('Mryantis ungustu (1'ig.).

STlilill1.1:

34. Strix flammed vir. migrescens. II, s

\section{EALANII)E.}

35. I'antion huliailus (Linn.)

31. Luten pennsylvanicus (11'ils.)

37. Timmunculus sparverius var. antillurum. (tim.)

FlitidTHA.

38. Freyata a'fuila (Linu.).

\section{I'HA HIIONII).E.}

39. I'haithan jlavirostris, Jiraudt ..

PELEI:ANII).F.

411. Pelecenus fuseus (Liun.)

IIIIIH.E.

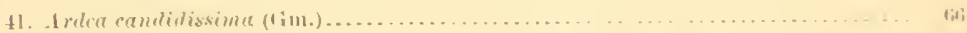

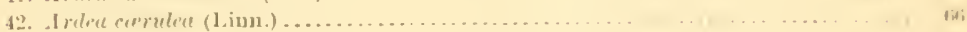

43. Butorides vireserns (Lim.)

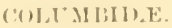

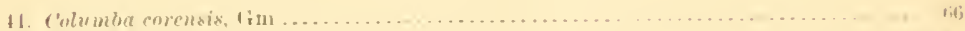

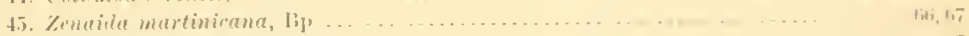

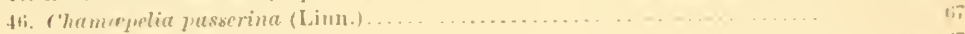

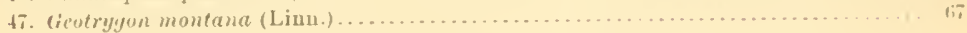

(II.II.IJIIII).E.

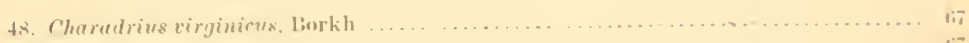

49. Strepsilas interpres (Liun.) . . . . . . . . . . . . . . . . . .

(1)LOP.ITII.L.

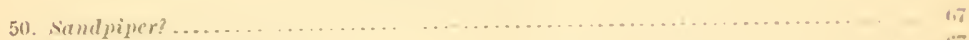

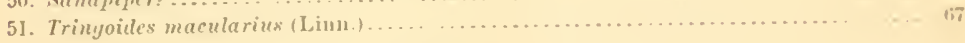

L.1I:II).E.

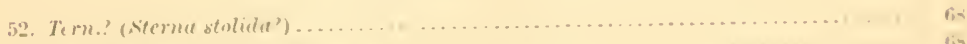

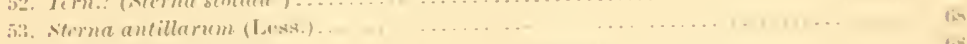

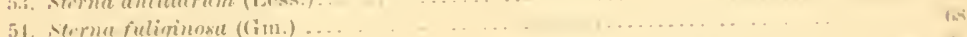

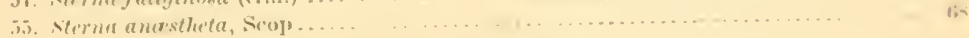

I'IRI'I:I.I.IIIII.K:

56. "Shinblutir. 
89.

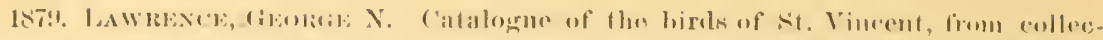

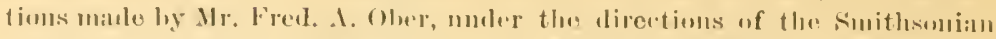

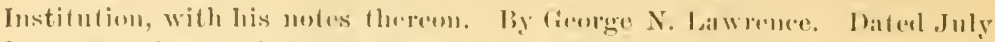

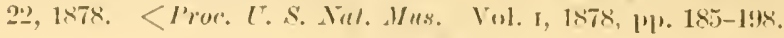

THIIIII).

1. Turdus nigrimstris, Lawr....... Jage.

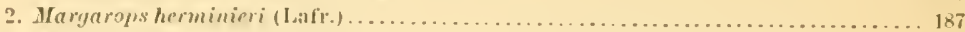

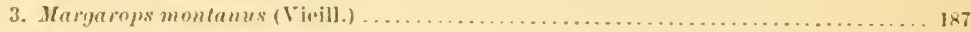

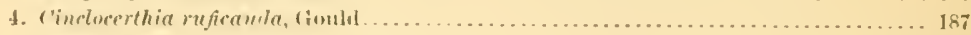

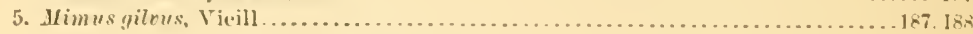

SYISIIISF.

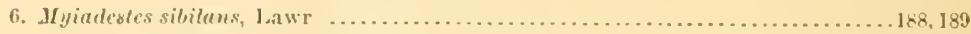

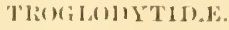

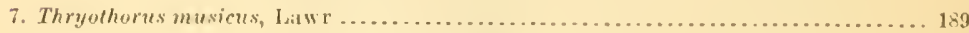

SYLVICOLIDE.

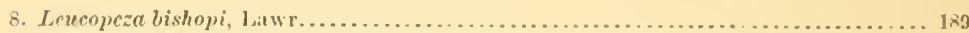

9. Sctophaga ruticilla (limu.) ........................................ 18?

VIRLONID.T:

10. Tireosylsia culidris var. dominicana, Lawr

HILUNHIXID.E.

11. Progne dominicensis (fiul.)

VELEDID.T

12. C'erthiola atrata, Lilwr.

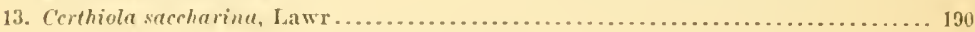

TANAGRIIE.

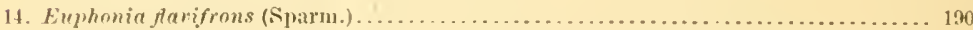

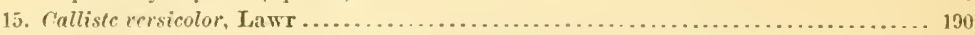

FIIINIILLIUE.

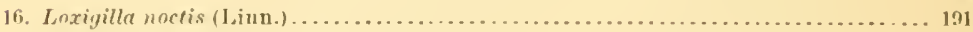

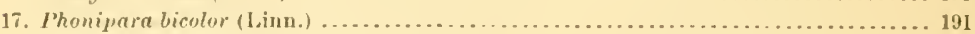

IC"T 1:R11).F.

18. Quiscalus —? 191

T'VI:ANI1).

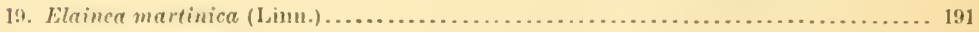

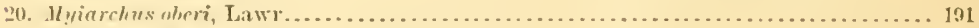

21. Tyranmes rodratus, sel........................................... 191

Tli(1) III,111.F.

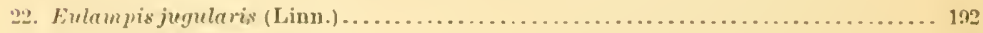

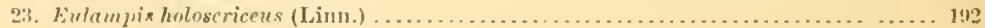

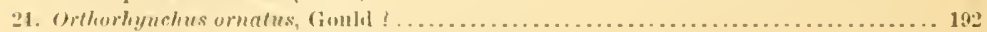

('YPSEIII).

Q5. I'holuta — : 
1879. LaWhexce, Glolige N.-Continued.

MI.CIUNII)E.

I.age.

26. Ceryle alcyon (Limn.) 193

('UC'T1,II).E.

27. Coecyzus minor (tim.)

28. Crotophaya ani, linu.

P'SIT'TAIIIDE.

29. ('hrysotis guildingi (Vigors)

$19: 194$

STRIIII)

30. strix flammea var. nigrestens, lawr. 194

FALC(III)E.

31. I'andion hatinätus (Limn.)

32. Buter pennsylvanicus (1Vils.)

33. Urubitinga anthracina (Nitze-I) ?.

FIEGATIIIF

34. Freguta a.juilu (Linn.).

PIAËTIIXIIN.

35. Phä̈thon athereus (Linu.)

J'ELECANIDE.

36. Pelccanus fuseus (Linn.) ......................................... 196

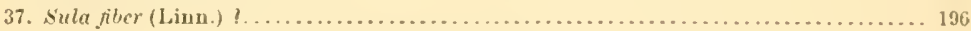

ARIMIID.

38. Ardea herodias, Lim ...................................... 196

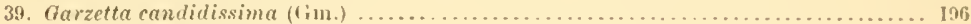

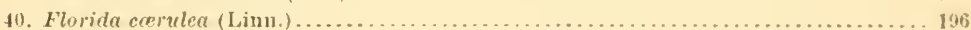

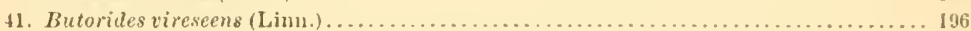

(')1.TंगIII).1:

42. Columba enreusis (fiu.). . 163

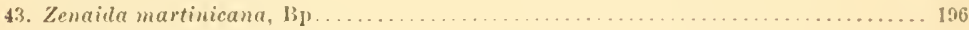

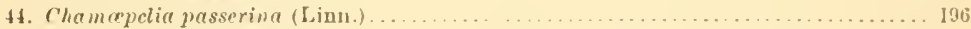

45. Geotrygon montana (Linn.) . . . . . . . . . . . . . . . . . . . . . . . 196. 197

IR.ALI,III.E.

46. Porphyrio martinicus (linul.)

('11 II.1I)liLIII.F.

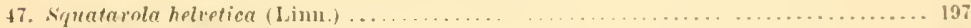

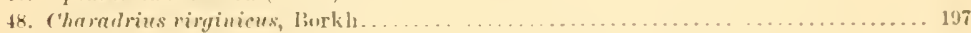

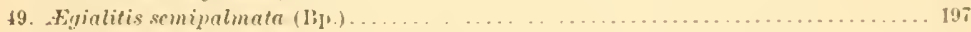

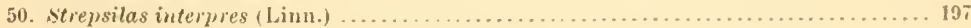

\section{GOLLOPAC'II).1:}

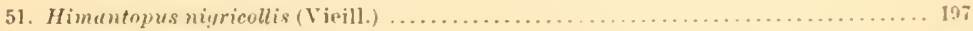

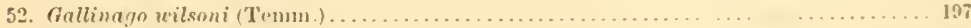

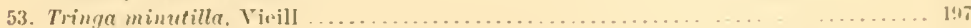

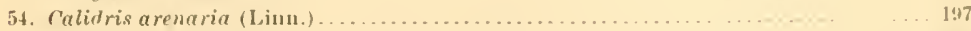

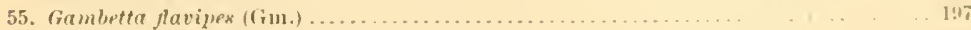

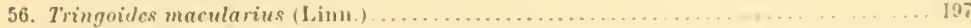

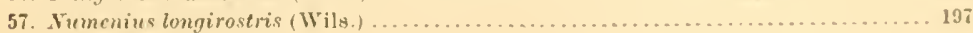

Bull. 40, N. II.—-6 


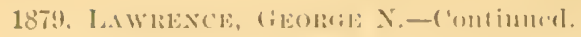

\section{L.1 I:111.T:}

Pagu.

5x. St.rna maximu, lsult

IPHICI'ITII).T.

59. I'vrieress -

\section{0.}

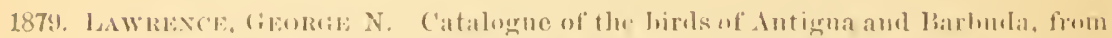

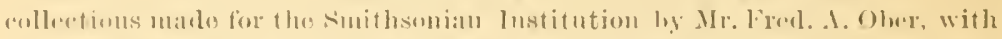

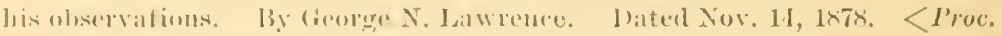

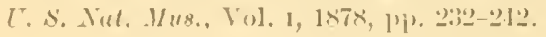

\section{A.TIIIIA.}

TURIII)

1. Maryarops densitustivis (Vieill.)

\section{SILICOLII) E.}

2 simms uerius (Bomid.)....

3. Sittrins motecille (Virill.?

4. Jemelrace petechiel (Limn.

5. Setophaya riticilla (Linu.)

\section{VIIEUNID.E.}

i). Vireosylvia calidris (Linn.)

\section{C.TREBII.E.}

7. Certhiola dominicana, Tayler. 233

\section{FIINGILLII).E.}

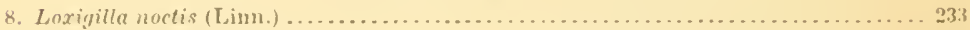

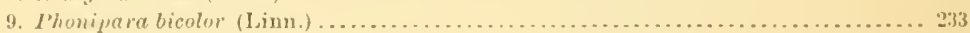

\section{TYR.INXIDE.}

10. Tyrammes rostratus, ,iel.

\section{THOCIIILIIT.}

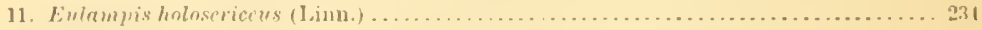

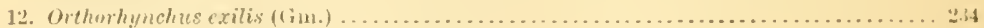

C'T'CL'LIII, T.

13. Coccyzus minor (tim.)

\section{STlilaID.E.}

14. Nijeotyto amaura, n. H

\section{F.IL(\%) (II),}

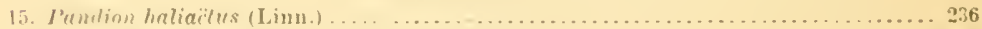

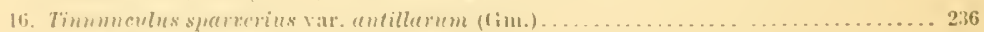

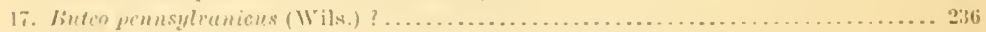

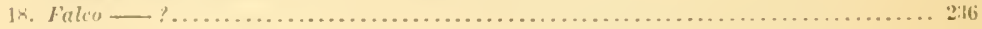

\section{FliE(XTIUE.}

19. Freyata aynila (Limn.). 


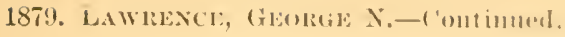

\section{AIIHEIIIS.}

21. Gurzetu candilissima (lim.).

2.). Horida corruler (Linn.).

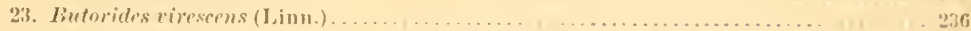

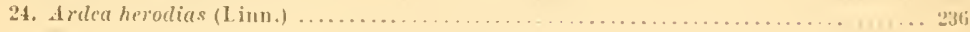

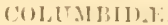

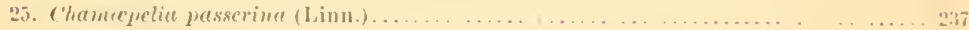

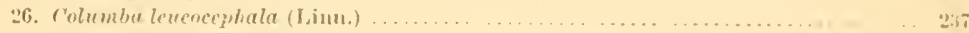

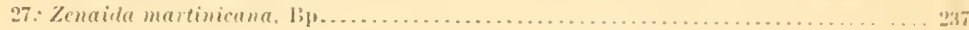

TETIRI).TIJ.T:

20. ()rtyx rirginianus (Lim,)

I:AJIAIIS.

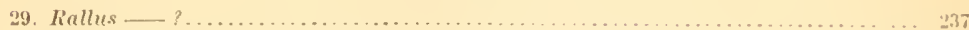

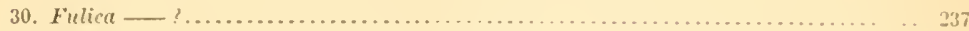

('I] IRAIIIII)_.

31. Charadrius riminicus, Borkh

\section{S(TLO)'ACIDI.}

32. Himantopus migricollis (Vieill.)

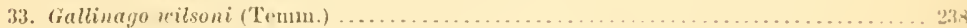

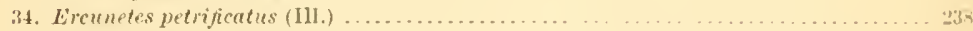

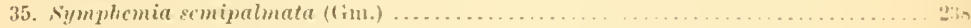

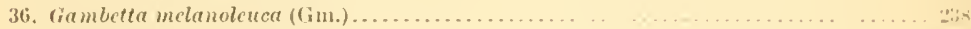

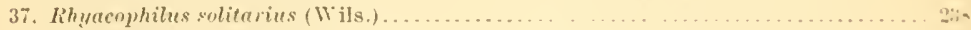

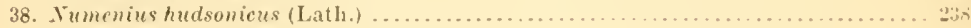

1.AliJD.E.

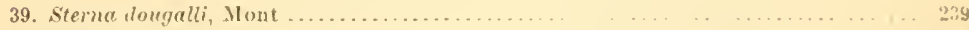

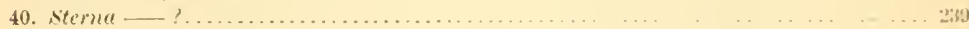

11. Larts atricilla (Jinn.) .................. . . . . . . . . . . . . . . . .

IN.TTIL.F.

42. Dafila bahamensis (Linn.).

liAkI3C1).

TLKI)II, E.

1. Cinclocerthice ruficauda, limbl.

SHTICOLIDE.

2. Dendruce pelechia (Linn.).

('EI:EISII).E.

3. Certhiole dominicana. Tas lar.

Flitirit.lut:

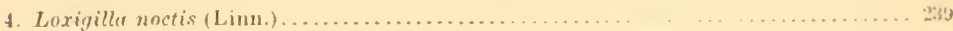

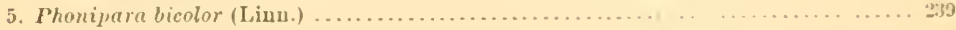

TYIIANXI1). E.

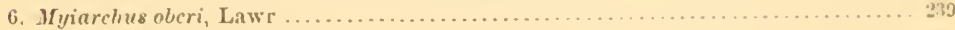

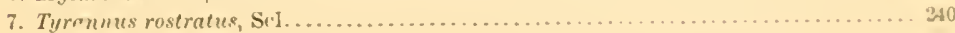




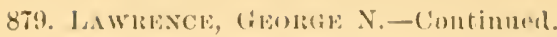

TIRO('IIH.II).F.

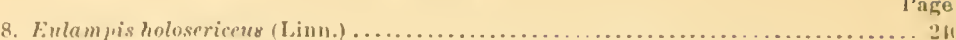

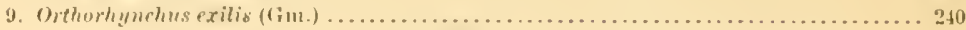

\section{IVITLII)E.}

10. ('oce!zus minm (fim.)

\section{FALC(INIIIST.}

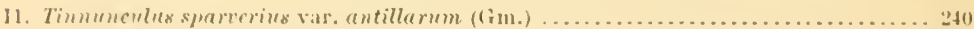

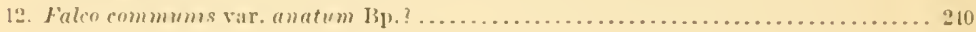

FIEGATII) F.

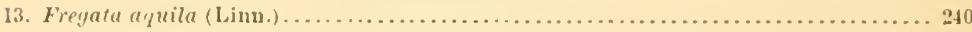

\section{IIIITIINIIE.}

14. Haïthon flarirostris, Iramit ....

PELECANIIDE.

15. Pelecanus fuscus (Linn.).

ARIDEIIIE.

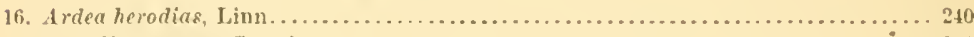

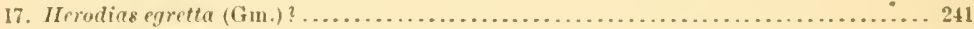

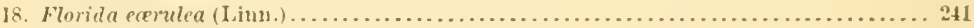

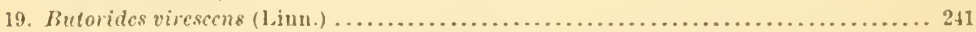

A $\mathrm{X}$ TIIS.

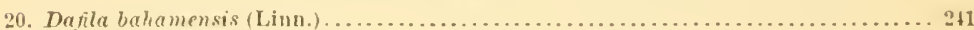

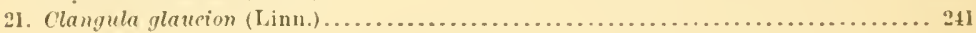

('OL. IIBH1).E.

22. Columba leucoepphala, I,iun 241

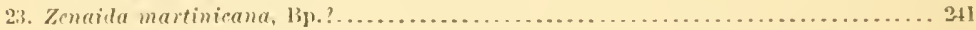

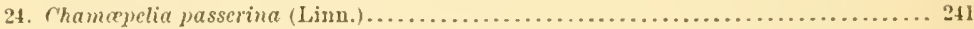

\section{NUMIIII).}

25) Trumita meleagris, Linn. Introduced...

Ii. ITIII) E:

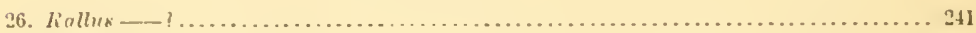

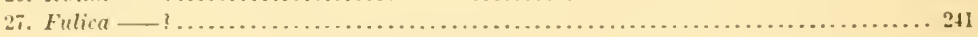

\section{FIIARAIIIII)E.}

28. Charadrius virginicus, Borkl.................................. 241

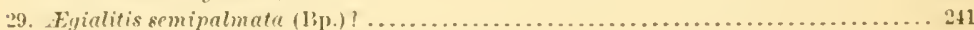

SC(HI, I'AC'ID.E.

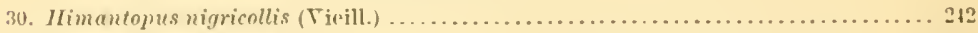

31. Gallinago wilsoni (Tomm.) . . . . . . . . . . .

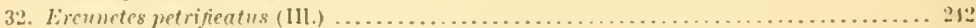

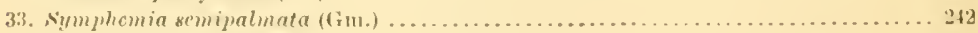

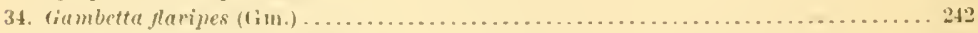

35. Thyacophilus solitarius (Wils.) ?................................ 242

36. Vumenius hudronicus (Lath.) ....................................

I. Alill). L:

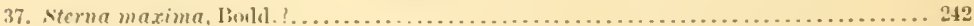

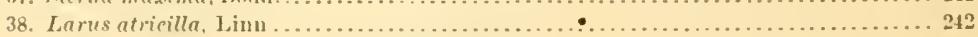

I')IIIITIII.F. 
91.

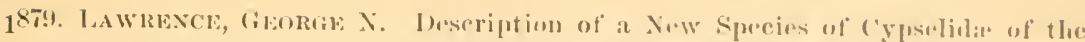

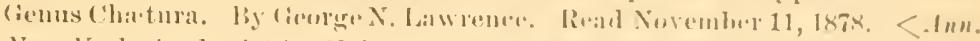

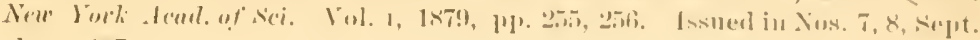
$-11 \mathrm{cec}, 1 \mathrm{~s} 7 \mathrm{~s}$.

1. ('hetsia slominisuna, n. .

Island nI Hominis'a, W. I

l'nga

92.

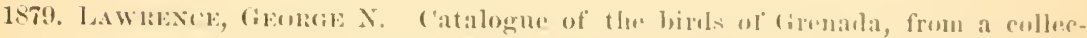

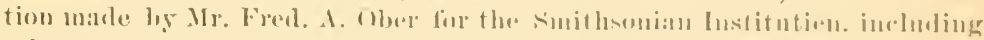

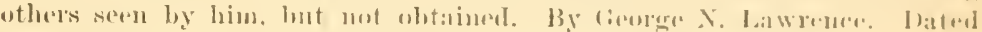

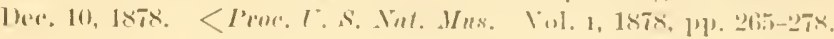

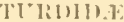

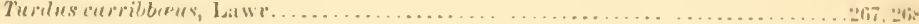

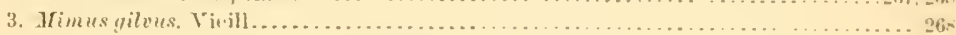

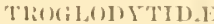

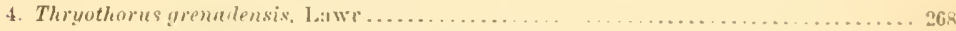

SII.VI(')L.II). E.

5. Setophana ruticilla (Liun.)

v1lionitis:

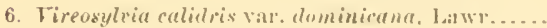

$.268,269$

IIIITIISIDH:

7. Proyne dominicensic (1)in.)?

('DliEliLI) F.

8. Certhiula atratu, Lawr

T. I IIIIIIT,F

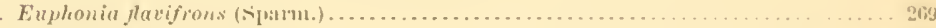

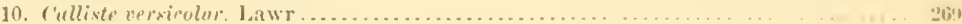

FliINiTLLII.I:

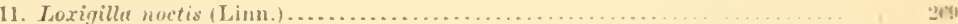

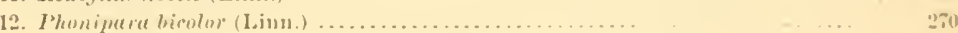

J"TElill. E.

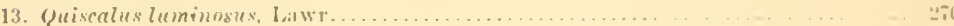

TYII.IXYIII:

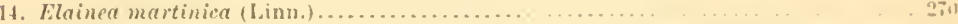

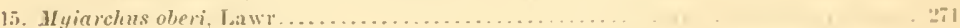

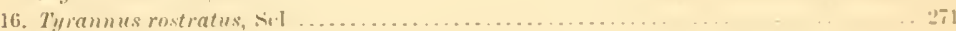

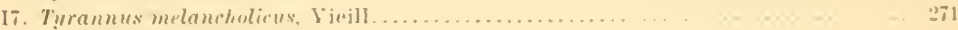

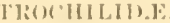

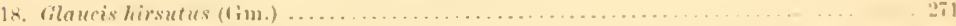

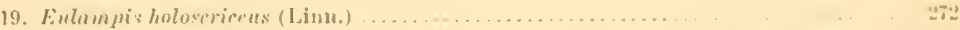

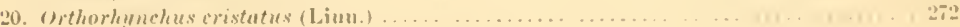

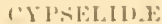

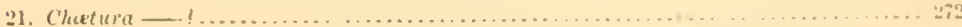




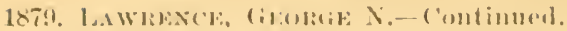

AI.THIIIII).

I'age.

22. ('ryle ale?un (Litun.)

CI\%? [.]1).1\%.

23. ('ureyzus minur (fim.)

272,273

2.1. C'rotomha!na ani, l,iun

ลัTนIกIII.E.

25. Nerix flammen var, ni!grescens. I.awr...

I:AL('II. II).E.

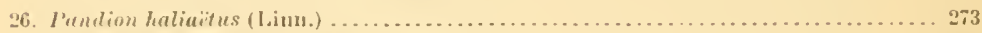

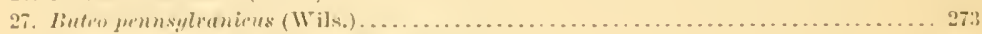

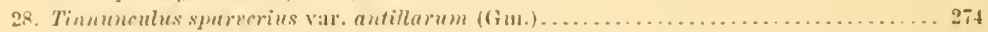

VIIECIATIUE,

29. Fregata uquila (limu.) 274

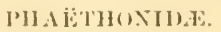

30. Phä̈thos atherews. Linn 274

\section{l'ELECANIII.}

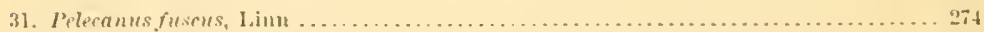

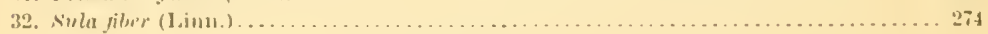

IIIFII).F.

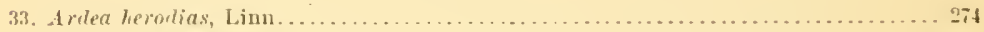

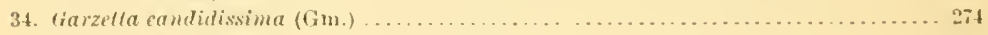

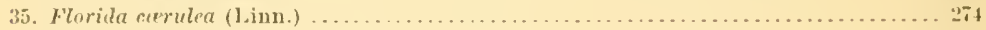

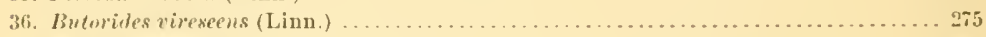

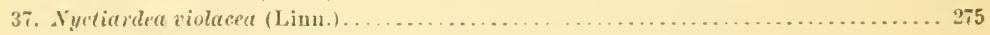

PLATALHH.

38. Plutalea ujaju (Iiun.) 275

(')I.T.MIII).T.

39. Columba eorensis, Gin ......................................... 275

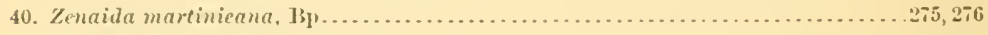

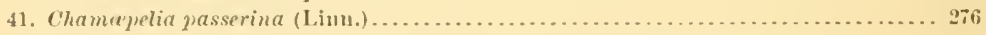

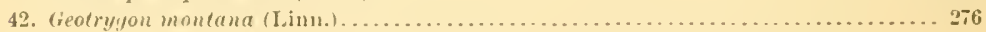

3:.11.1,11). E.

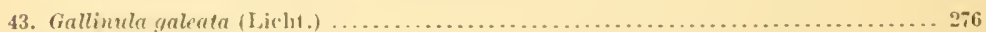

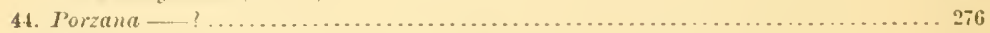

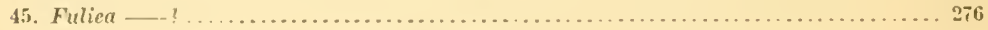

('IIALAIIRII).

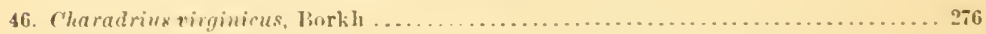

SCOL(1).IC:H).E.

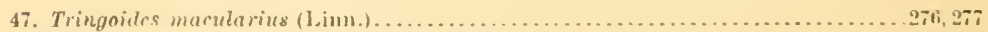

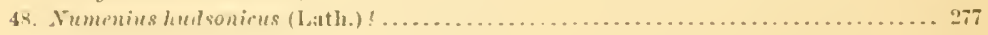

\section{I.AlIIII.}

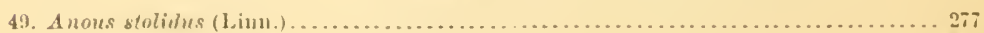

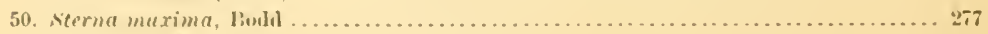




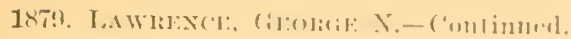

51. Neme dounalli, Mant

52. Nerma fulininessa, (im

5:. Letres uticillu, Limt.

I'(1)19.11.1\%111.,

51. I'oricigs ?

'111: (ins

1. Fumerpelie passerina.

2. Zemuidu murtivierance.

3. Quiscales lumimon:

1. F'neyzus minor...

5. ('mthimler - !...

6. Phonipara bieolu:

7. Mimus viluus.

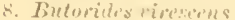

9. But on penusylmenicus.

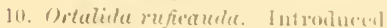

11. ONtyx viryinianus. Introsluxwd

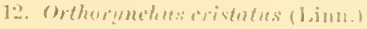

93.

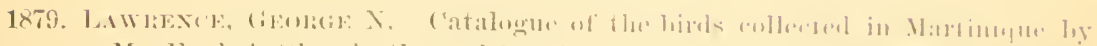

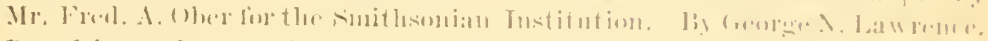

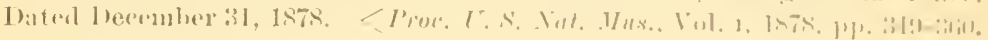
THI:III).

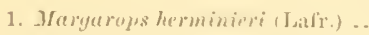

2. Maverops deusirustri; (Vteill.)

3. Jargurops momfanm" (Vieill.)

5. Ramphecisclus brechyurus (Vicill.)

SIITIIII:

6. Muiadeste's geniburlis. siw

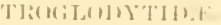

7. Thruthorus murtinicensis, sil

SIL1(1)I.111.

8. Demboes ruñgulu, Bairal

9. Setopleser rutieillu (1,inn )

VHRE(II) I:

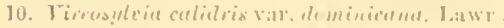

JIIRI XIIXIH]:

11. Prome dominicensis (t,m),

('):RE:BII.1.

12. Certhislet martinirnmu. lividli....

I.I.T.MiliL11.1:.

14. sitlater ynadrlowpensis, Lathr 


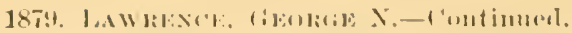

\section{FilNiII,III,K.}

15. Loxigillu nowtiv (Limu.)

16. Plonipara bioblor (Linn.) ...

11"li:lill.

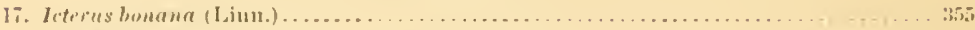

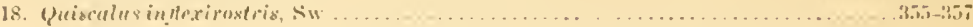

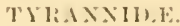

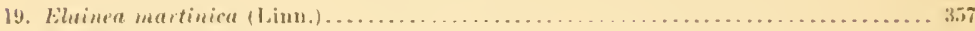

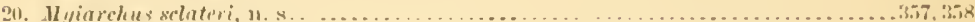

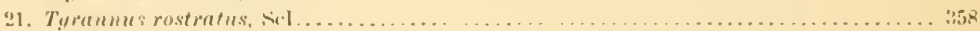

TI:U'III.I1H.X.

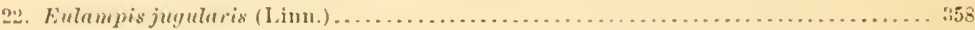

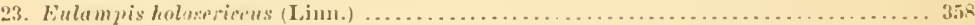

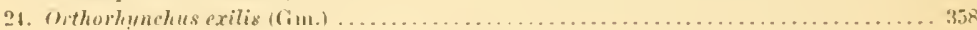

('YPEEIII).F

25. rhoturu —?

A I.'EIIXII)..

26. Cermle aleyon (Limm.) $\sin 9$

('TOILIITE:

27. Coecuzus minor (Gin.)

\section{FALCONIIF.}

28. Tinmunculus sparucrilus var. antillarum (Gim.)

FIERATIIDE.

29. Fregata aquila (Limu.)

\section{PII HTIUNIDA.}

30. Phä̈thon dlavirostris, Brandt

JPLEKANIDF:

31. Pelecanus fusens (I.imu.).

\section{AliJ)lll.}

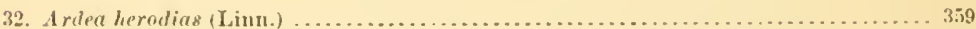

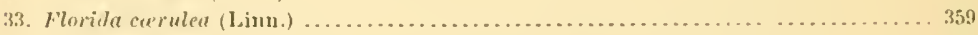

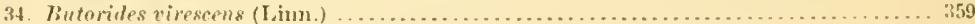

(1)1.1.MI).1:

35. C'olumbre corensis (fim.) . . . . . . . . .

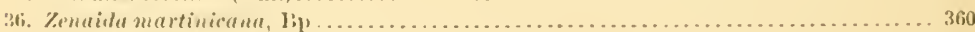

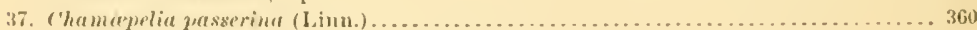

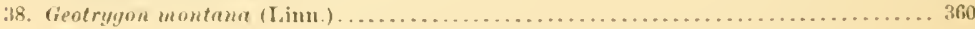

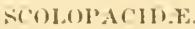

39. Tringoiles marulurus (Lim.) 360

I. I I:III.F.

40. Nterna dougalli, Mon! 
94.

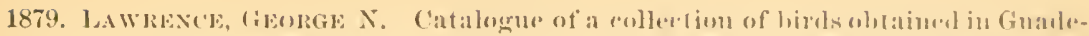

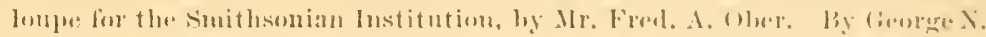

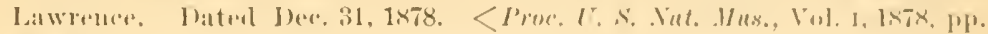
$4.19-462$.

\section{TIIIII):}

Page.

1. Maryarops heminieri (Lafr.).

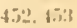

2. Maryurops densirostris (Virill.) 15.3

3. Jucreareps mesntanus (Vicill.) 4.i:3

4. ('inclocerthia rujicauda. (iomld 15.3

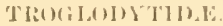

5. Thryothorus rufesefus, Lawr.

SILII(UII).E:

6. Siurus norius (lbold.)

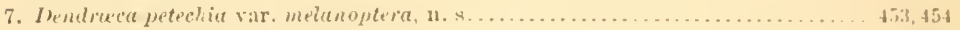

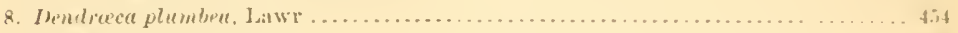

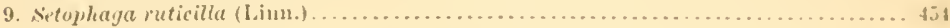

\section{VIIEONII).F}

10. Tiresylia calderis var. duminicene, Lawr 454

HIITIDIXI11.F;

II. Hirundo horreorum, Inarton

('LIEISI).

12. Ferthiola dominicana. Tụ̂lor

TAXAIIII).

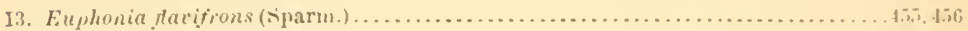

14. Naltator guadeloupensis, Lafir.....................................

FIINIILLIIIE.

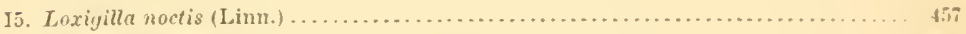

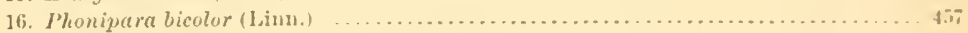

[('TEI:II),E.

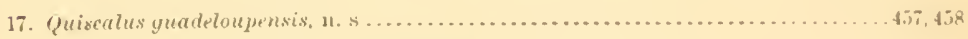

TYIR. XNII.F.

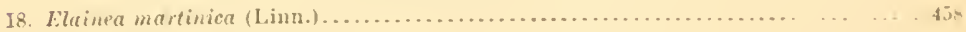

TH)(HLID.E.

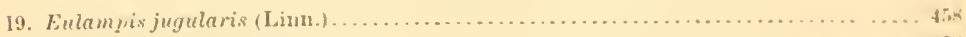

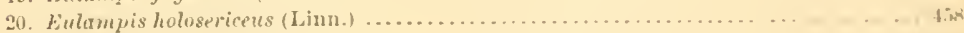

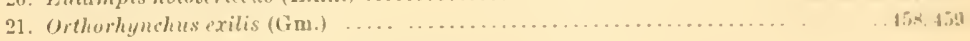

('Y I'SELID.F.

22. rypsolubiles niyer (Tim.).

I.CEUINID.E

23. ('eryle alcyon (Lim.) .....

24. C'eryle toripuata (1.inn.)

['II'II).:. 


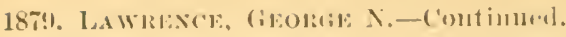

CI'TIII.K.

2f. ('uecyztls minur (lim.)

\section{FAI.(i)}

97. Tinnumeulns sparverius var. antillarum (diu.) $.459,460$

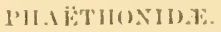

28. Häthun whereus (Jiun.)

Ali) E.11).J.

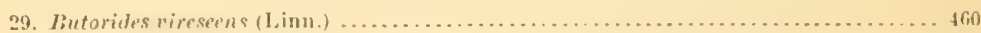

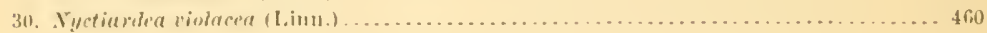

(1)L, IIISH).

31. Columba enrensis, Gm ...................................... 460

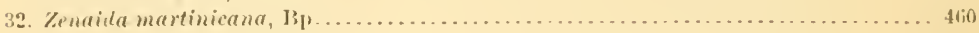

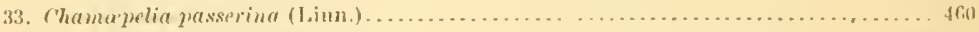

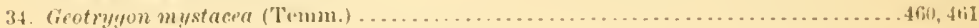

RA1.1II.T:

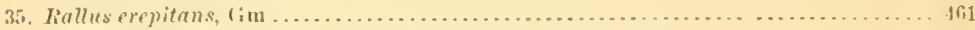

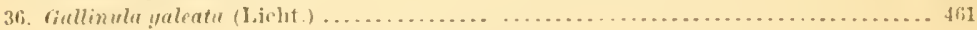

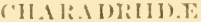

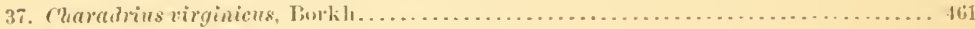

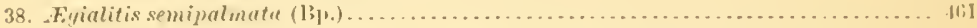

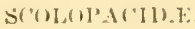

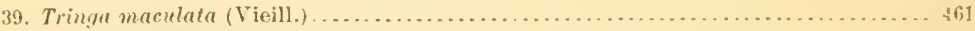

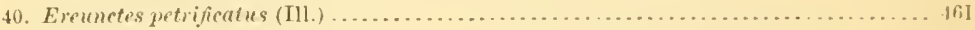

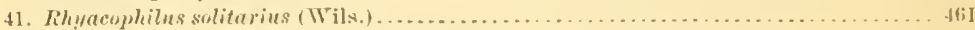

\section{L.1RIII.}

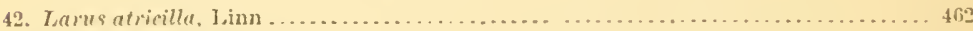

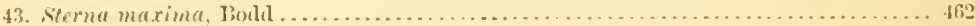

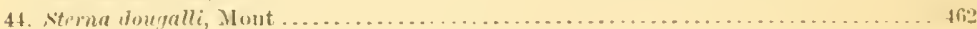

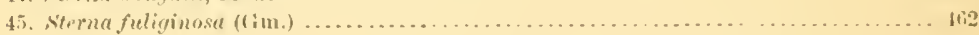

95.

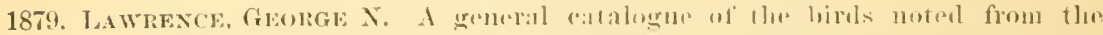

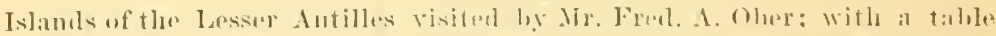

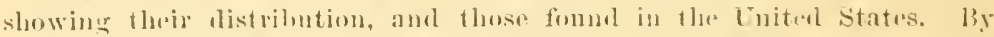
George N. Tawrenew. Datod March 20, 187!. <Mros. T. S. Tat. Mus., Vol. I, 1878,12$] .486-18$.

\section{JHUW (WF TIIE L.FSSIR ANTILIES}

Page.

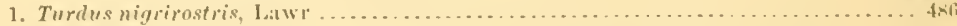

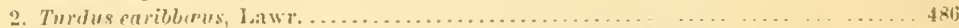

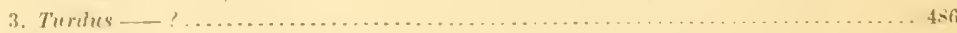

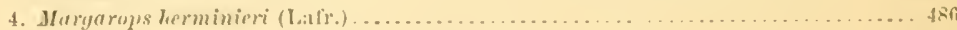

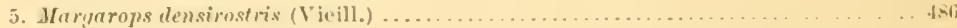

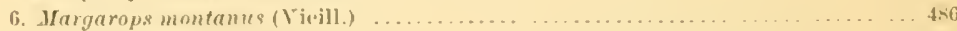

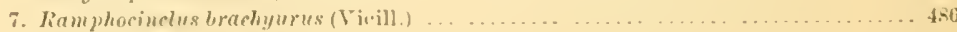

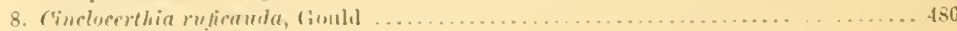

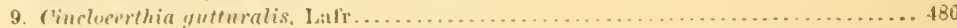




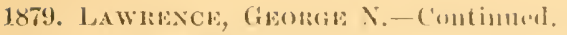

Iage.

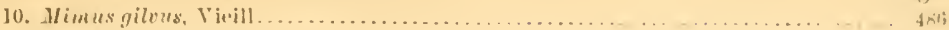

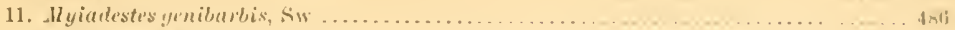

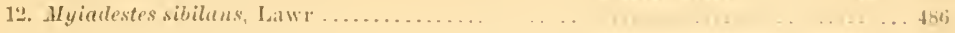

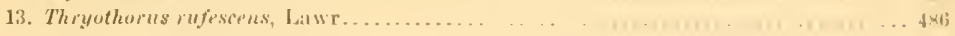

14. Thryothorus musicus, Law r................... 4.6\%

15. Thryothurus grenadensis, Lawy ......... . 186

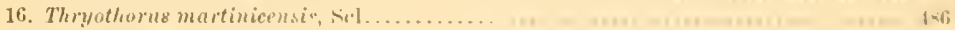

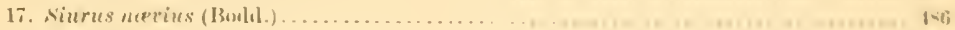

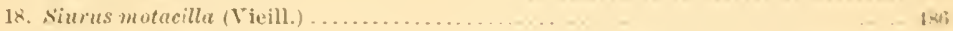

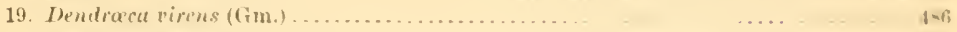

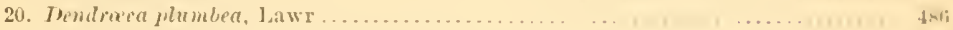

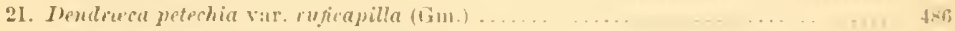

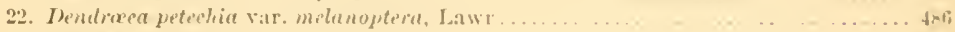

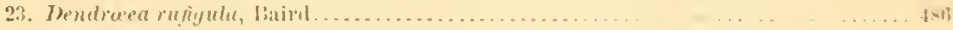

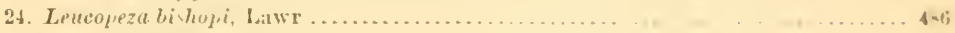

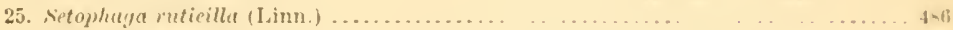

26. Vireosylria enlitris (Lim.) .............. ... . . . . . ..... isli

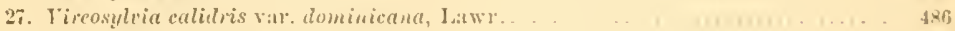

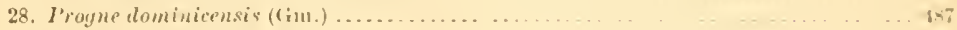

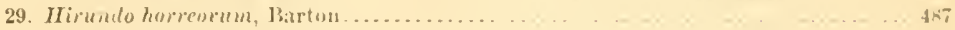

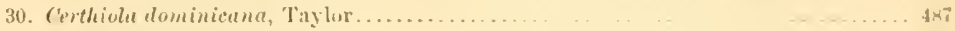

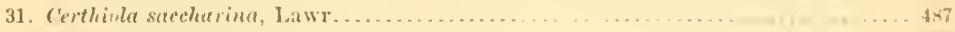

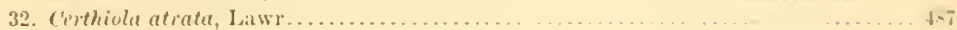

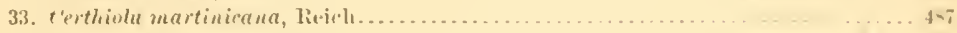

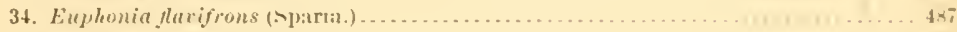

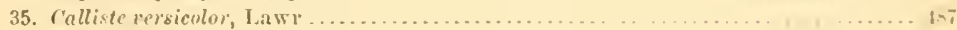

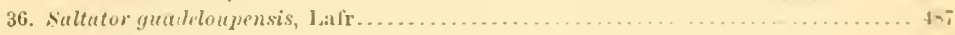

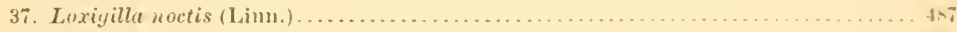

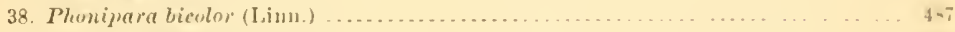

39. Quiscalus luminosus, l.awr ........................

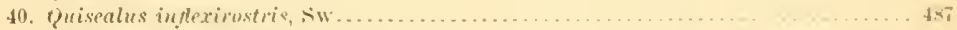

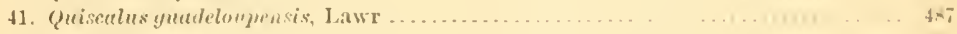

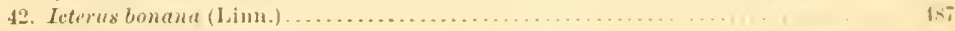

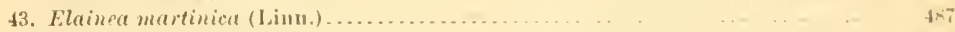

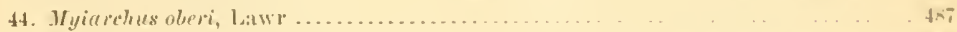

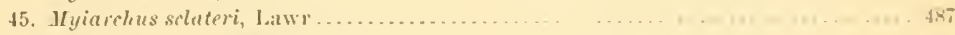

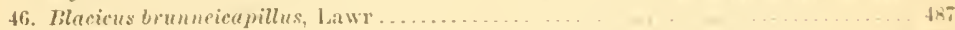

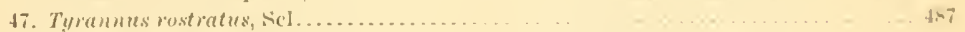

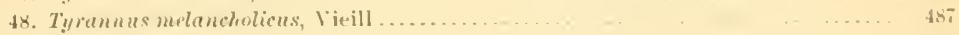

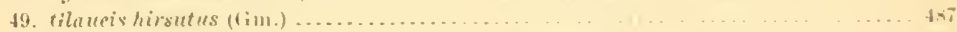

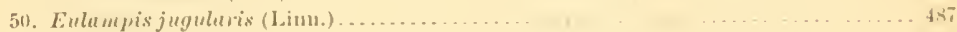

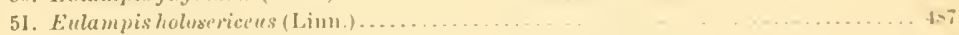

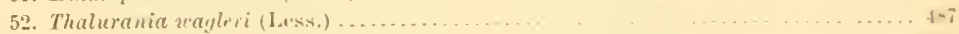

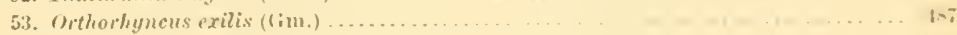

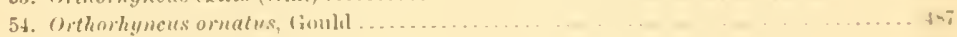

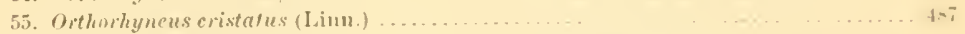

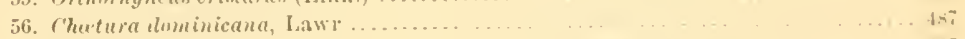

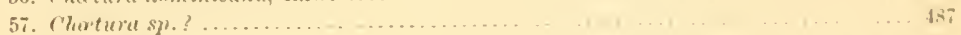

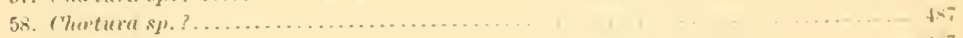

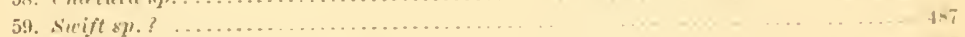

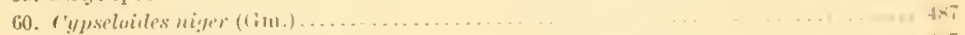

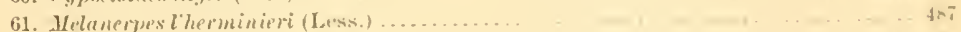

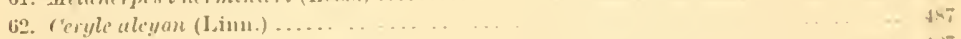

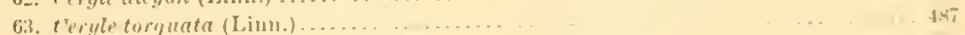

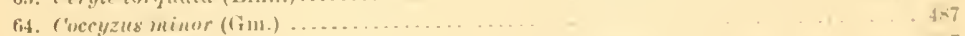

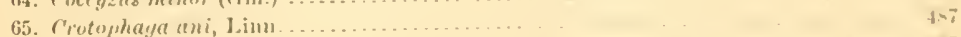

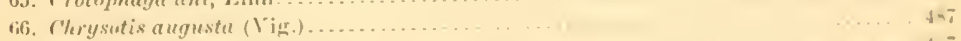

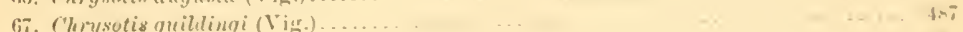

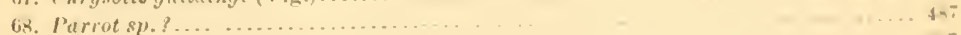

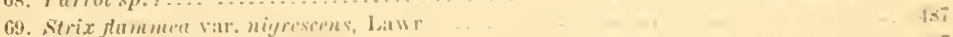

70. speotyto amaura, Lawr............

71. Pandion haliertus (Limn, )........

72. Buteo penusyleanieus (Wils.)....... . . f-i

73. Trubitinge anthracina (Xitzch.) ?.... ...... tmi 


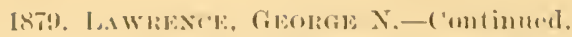

I'age.

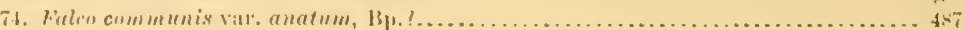

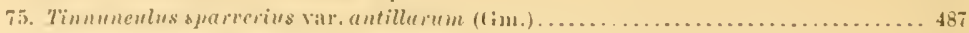

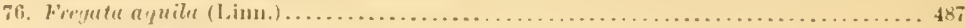

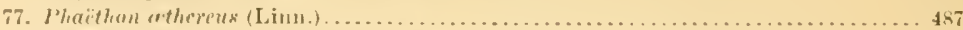

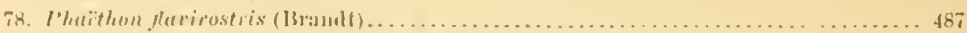

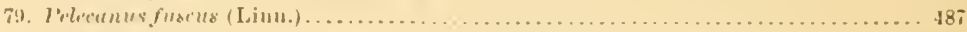

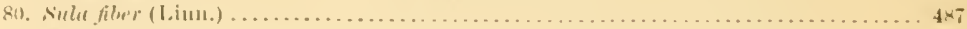

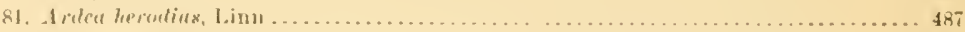

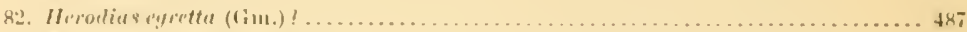

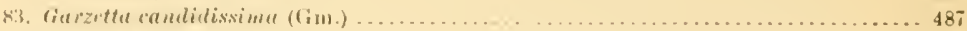

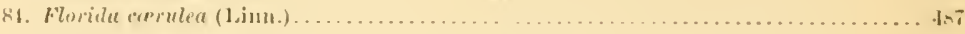

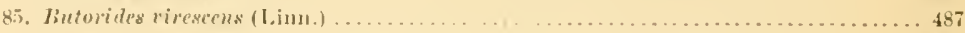

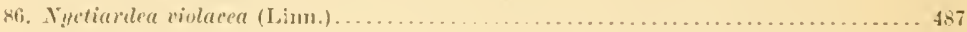

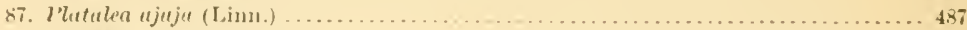

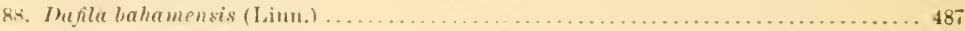

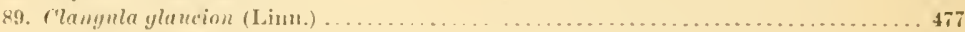

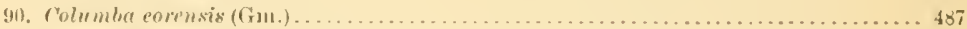

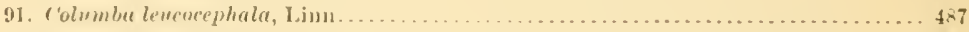

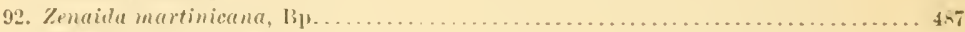

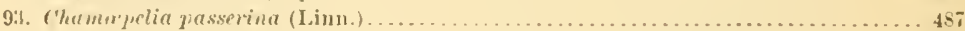

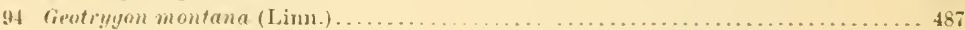

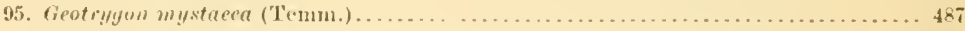

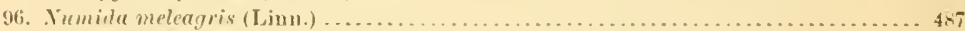

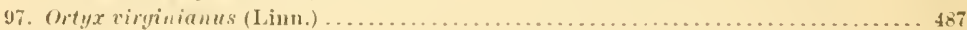

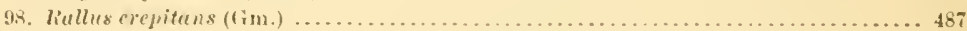

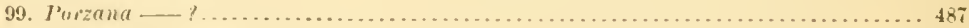

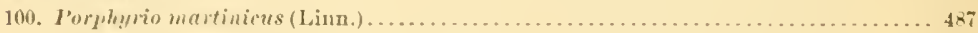

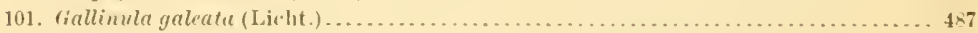

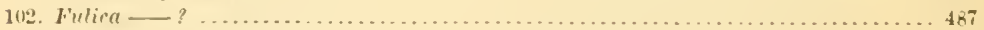

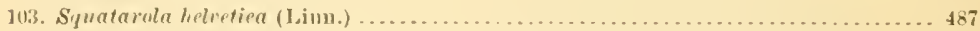

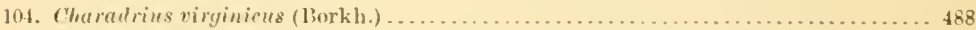

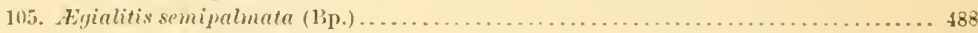

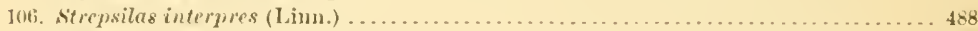

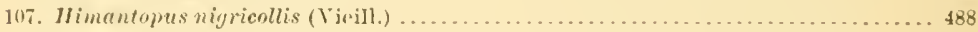

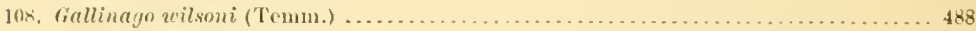

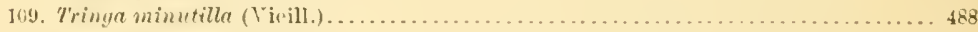

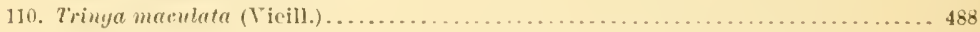

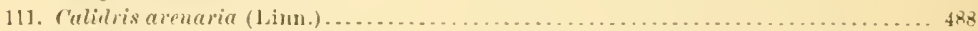

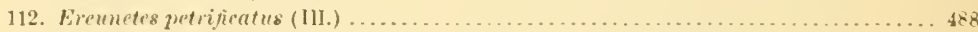

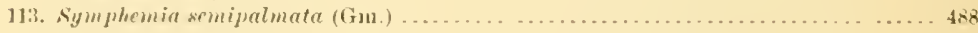

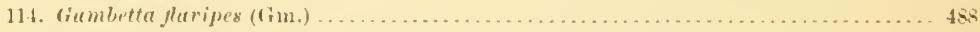

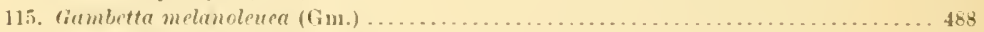

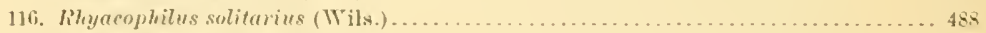

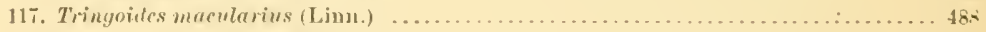

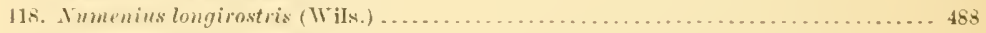

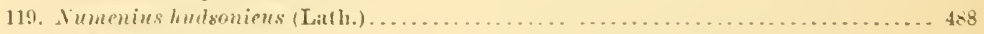

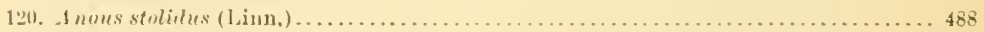

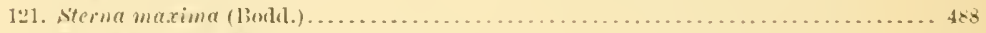

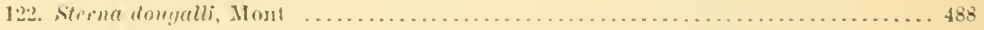

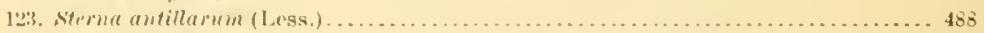

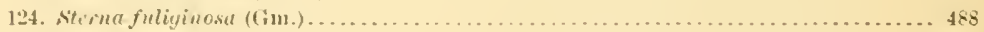

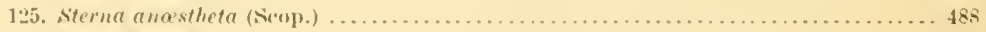

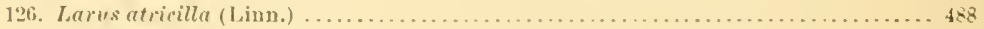

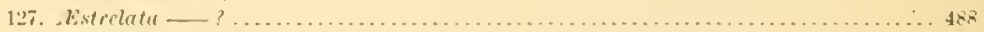

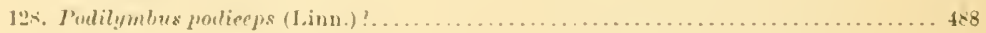

96.

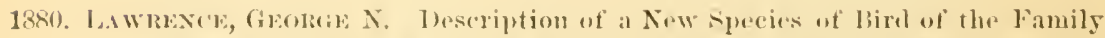
'T'urlida, from the Island of Juminisa, W. I. By Geo. N. Lawrence.

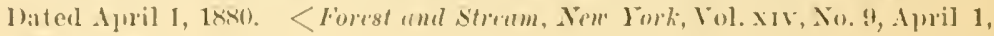
$1 \times \times 10$. 1. 16i.. 
97.

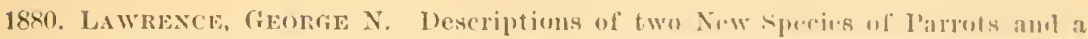

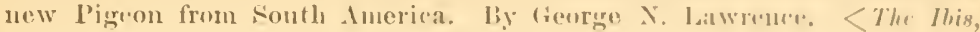

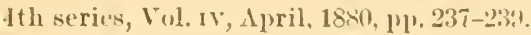

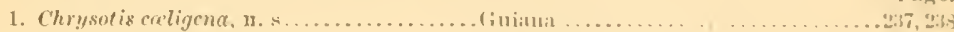

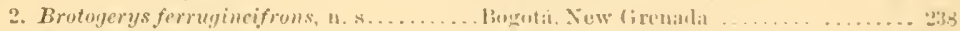

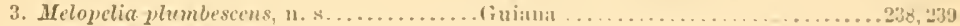

98.

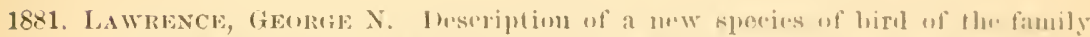
Turdida, from the Island of lominiea, W. 1. Jy Lan. N. Lawronee.

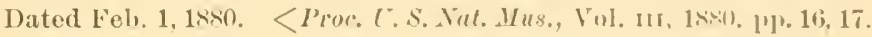

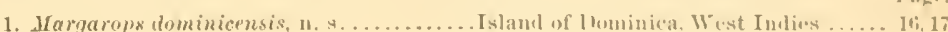

\section{9.}

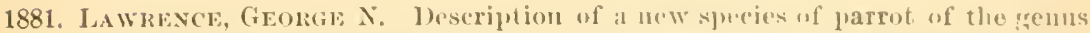
Chrysotis, from the Island of Dominira. By (inorge $\mathrm{N}$. Lawrence. Ditted

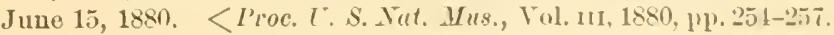

ISLAND OF HOMHICA. WEST INHES.

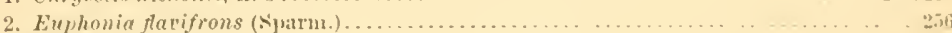

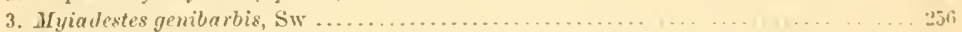

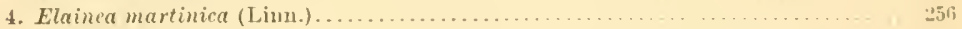

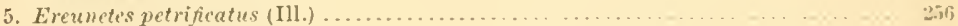

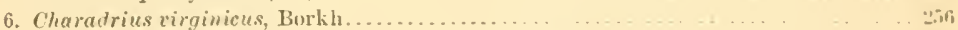

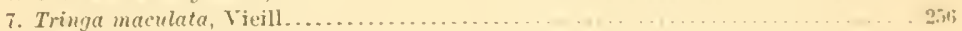

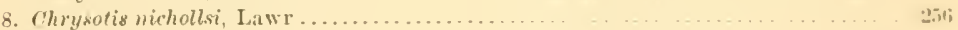

9. Nyctiarlea violacea (Linn.). ..................... . 25

10. Charudrius rirginicus, borkh .................. . 257

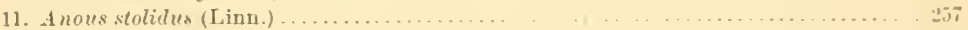

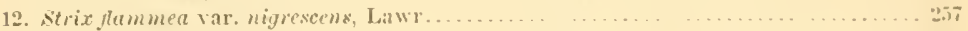

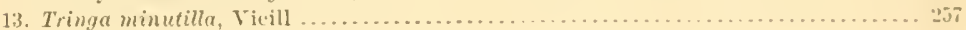

100.

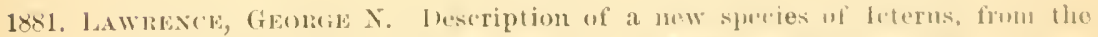

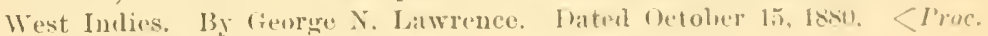
C.S. Nal. Mus., Vul. 11, 1880, $\mathrm{p}, 3.31$.

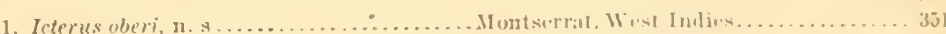

\section{1.}

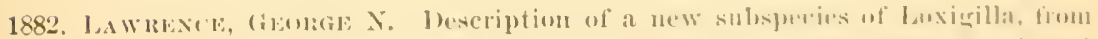

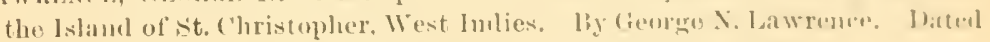

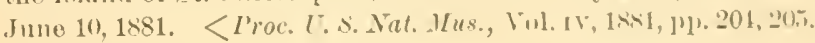

Page.

1. Inxigilla portoriecusis vir. "lrandis, $и$.

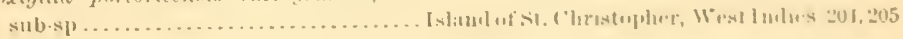

\section{2.}

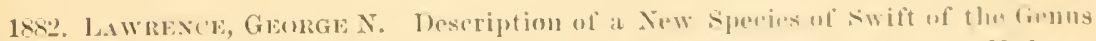

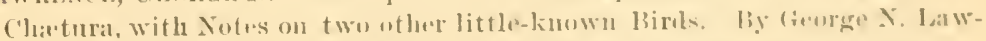

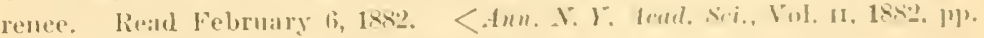

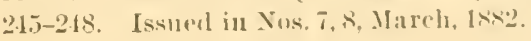

1. Chatura gaumeri,u. . ............... Yuralın 


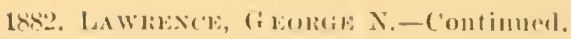

Nitris.

1. I'yrangu rosmigularis, Cabot ............ Page.

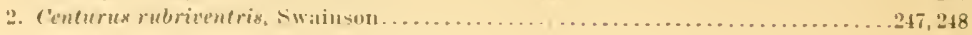

103.

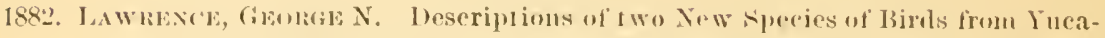

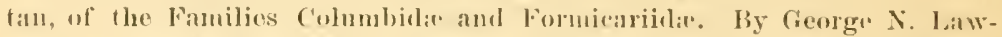

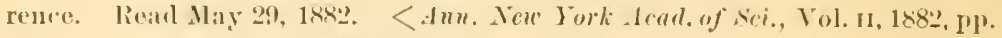
287, 288. Issued in No.!), دlay, 1882.

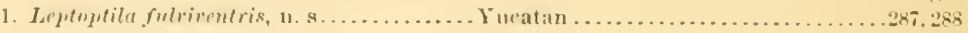

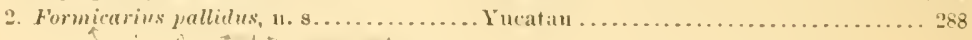

104.

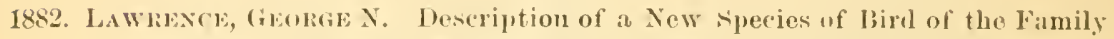

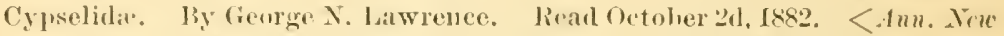
Fork Acul. of sci., Fol, 11, 1882, l'p. 355, 356. Issued in Nos. 10 aul 11, 1)ecember, 188:2.

I'age:
355,356

\section{5.}

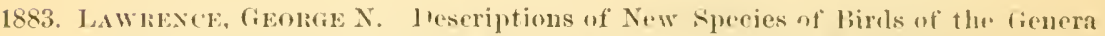
('hrysutis, Furmicivora, and spermophilis. Bý George $\mathrm{N}$. Law rence. Read

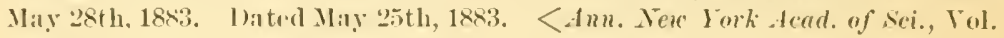

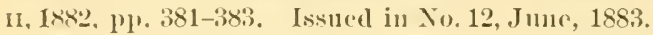

Pago.

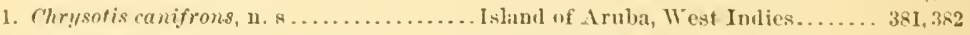

2. Formicivora griscigula, 11.8 . ........... Brit ish Guiana................. 382

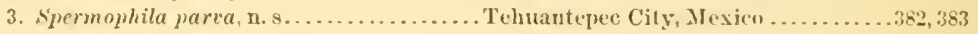

\section{6.}

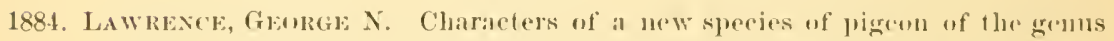
Engyptila, from the Island of Frentala. West ludies. By Grorge X. Istwrence. <The .luk, Vul. 1, Xo. 2, April, 1881. 111. 180, 181.

l'age.

1. Engyltila vellsi, n. s................... Islanwl of Grenada, West Indies......180, 181

\section{7.}

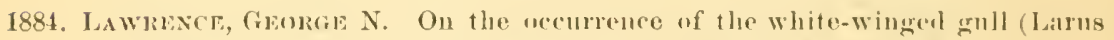
lencoptersis, Faher), in the State of Now York. By George N. Lawrence. <The Aluk, Vol. 1, No. 3, July, 1881, 11]. 210-212.

1. Larus lineopterus, Faber.

1'age.

1'eterloro, N. Y

$.210-242$

108.

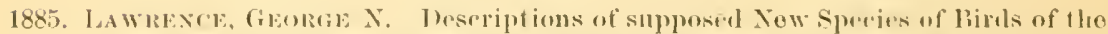

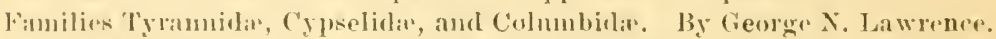

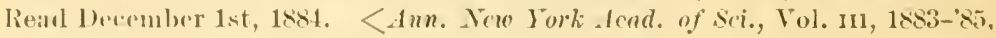
pp. 156-15x. Insued in Nos. 5, 6, Felnuary, 1885.

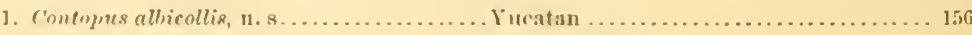

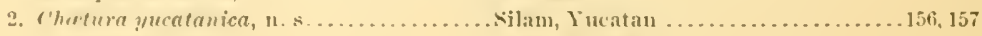

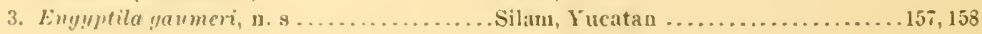




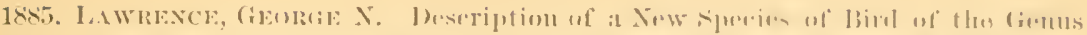

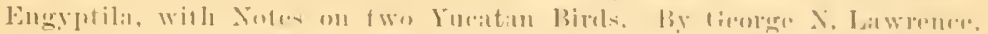

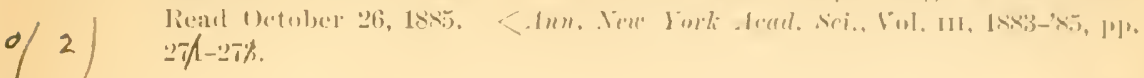

1. Enypptilec rinaceifulve, 1 . :

liage.

2. Enfyptila julvirentots. Latw2

Tromax, Yne:al:m

271. $27: \div$

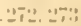

3. Clecture gummeri, Law? .............. Furitan . . $2 \pi ;:$

110.

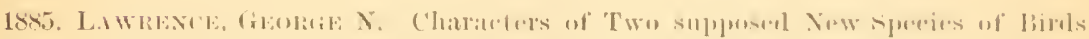

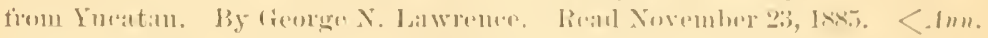

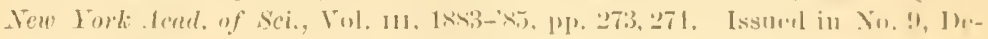
cemler, 18xis.

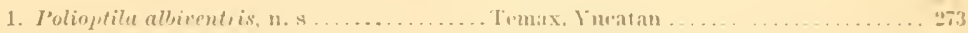

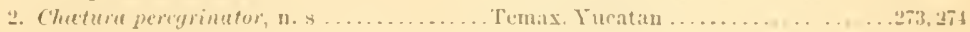

111.

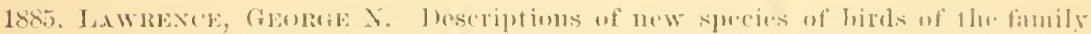

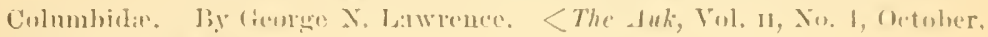
1885, 111, 357-35.9.

l’ome

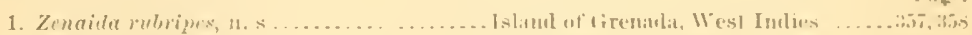

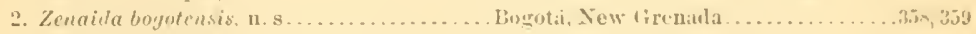

\section{2}

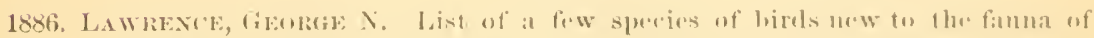

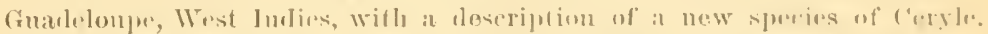

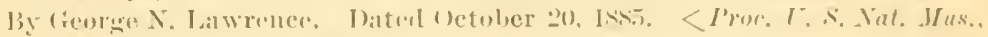

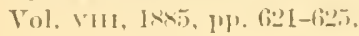

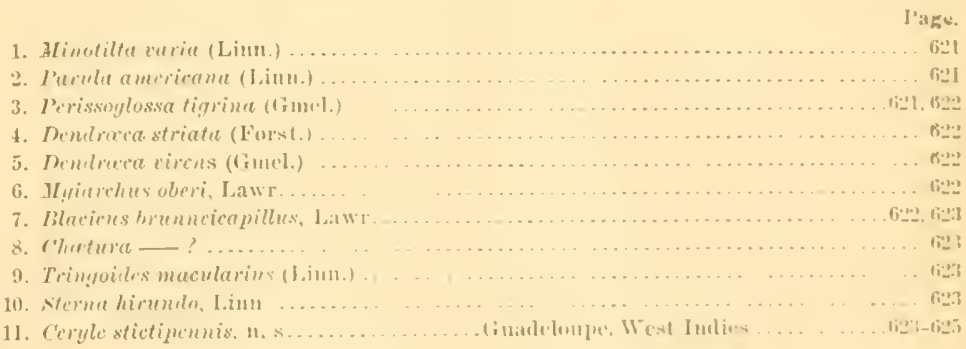

113.

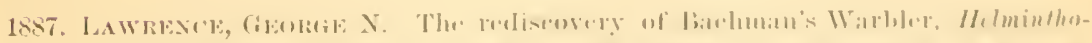

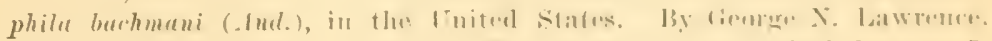

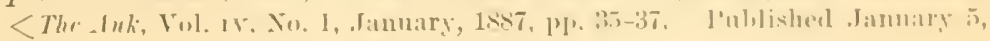
1887 .

I'age

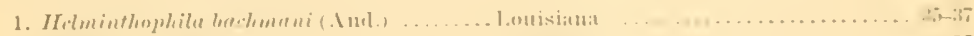

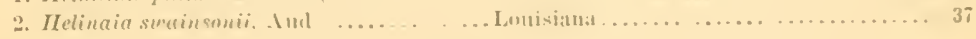




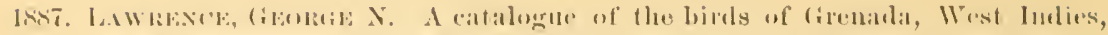

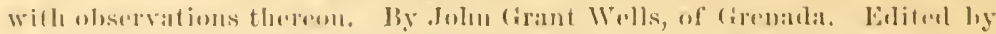

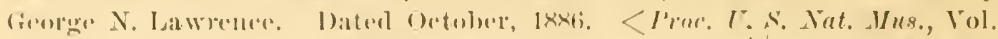

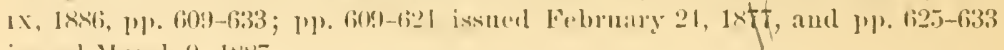

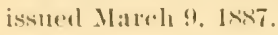

TITIIII.L.

1. Turlus migrirostris, litw.

l'age.

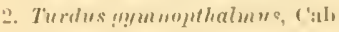

3. llimus yileus, licill

Thu(i)[MTII):

1. Thryothones yrenade'nsis. Law $\mathrm{r}$.

MNIOTILIH.E.

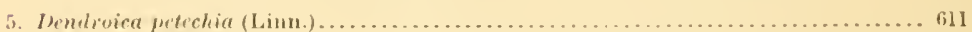

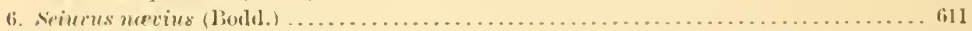

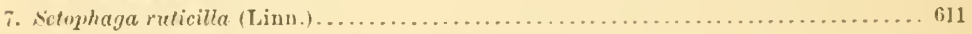

VIRE(INII).E.

8. Tireosylviu calidris vat. duminicana, Lawr.

HIIIXIIXII1).

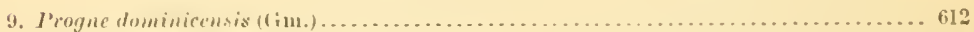

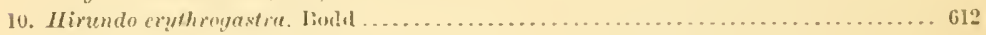

C(EIREIBI.E.

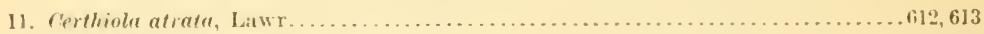

12. Certhiola saccharina, Lawr . . . . . . . . . . . . . . . . . . . . . . . . .

TANAGHIITH

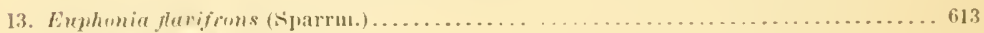

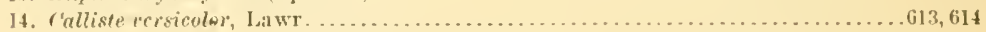

FIINTIILIII)

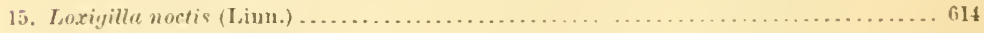

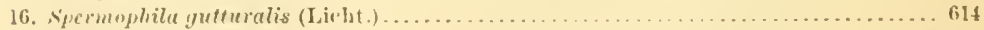

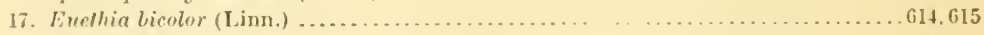

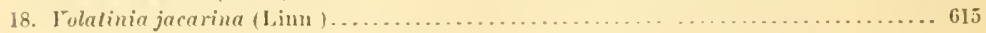

II"TEIII).F.

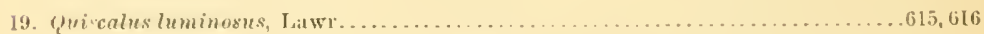

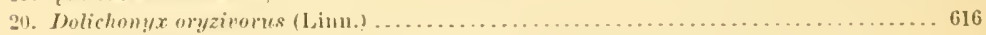

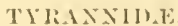

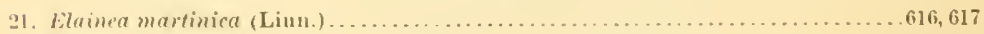

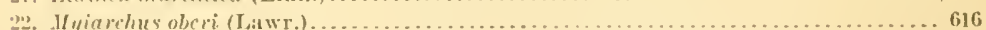

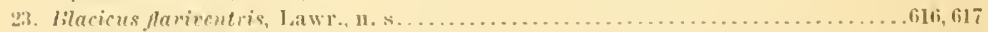

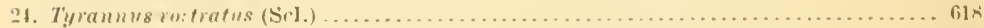

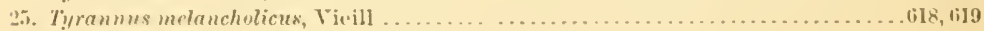

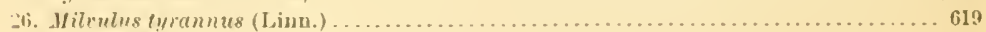

TH() HH,ID.E

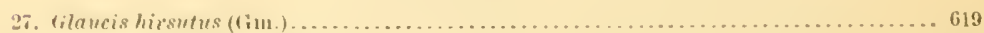

18. Eulampis holosericeus (Linn.) .................................... 619

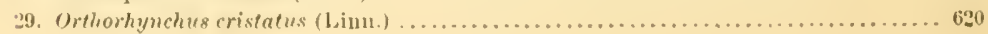


1887. LaWreNCl: George N.-Continner.

('Y]'TH:I, II)E.

30. Chutura rineretemtris, sil

Page

31. Hemiprocne zonaris (Sh:1w)

(5)-11

6211

IL. KIIXII, I.

32. C'crylc alcyun (Linn.)

(1\%(YI.II).E.

33. Coecyzus minor (1im.)

34. Crotophaga ami, Linı

NTIIIII).E.

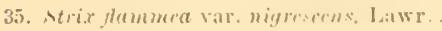

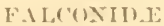

36. Fitle, culumbarius, limu...

37. Tinuusulus sparverius caribhartum (tim.) .

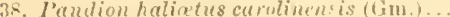

39. Butero latissimus (Wils.).

40. Regerhinu? "ncinatus (Temm.)

(')],T IIBII).

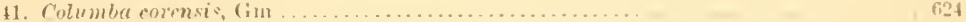

42. Zenaila martinicamu. Bp ........................ fizt

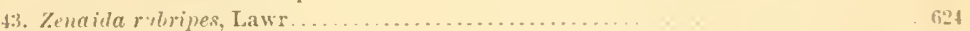

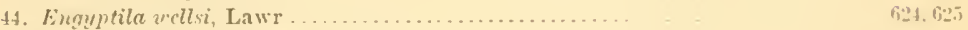

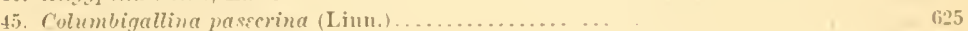

ti. Geotrygon montand (Linn.)....................... - $1: 25.626$

AIDEIDE.

47. Ardea herodias, Linn.

48. Irdea candilissima, fim

49. Ardea coerulca, Linm.

$6: 6$

50. I re?ca vire crns, Lim

1026

51. Nycticorar violacens (Limn.)

PLATILFIIIF.

52. Ajaju rosen (I.imm.)

('II.II.1DIIII).F.

53. r'huratrius dominieus. IIill .

51. ('haralrius squatarola (Linu.).

5.). Egialitis somipulmate, linnap

56. Oxyelues rociferes (Linn.)......

\section{STI,EI'SIIIII.E.}

57. Nirepsilas interp)es (I.inn.)

58. Gallinetyn delicata (1)mat)

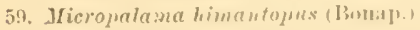

tio. Actodromas macwlata (Via.ill.).

61. Eremetes pusillus (lium)

62. Limosa fentua (Linn.) ...

63. Symphemia semipalmata (Giuel.)

6f. Ictitis maeularia (Linn.)......

65. Iartramia longicauda (Bechst.).

Bull, 40, N. M.—— 


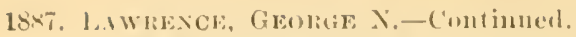

i6. Totanus ftaripes (binel.).

(i7. Totanus inelmolruces (timel.) ..

bir. Numenius hulsomicus (Lath.)

69. Vumenius borerelis (Forst.).

1:.11.1.11).E.

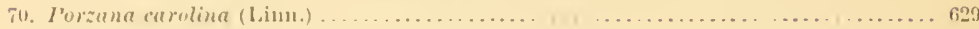

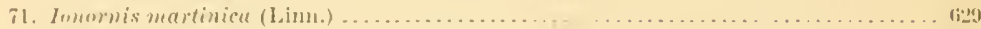

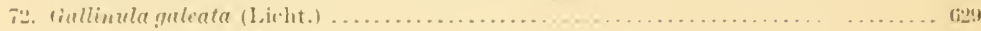

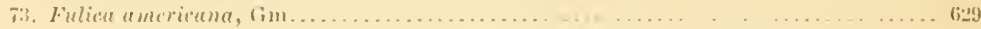

A. КTII.E.

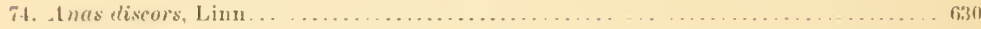

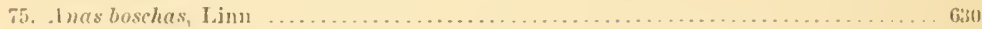

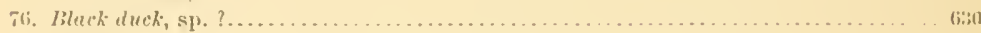

7i. Erismaturt rubida (Wils.) . . . . . . . . .

IVLEG.TTIDE.

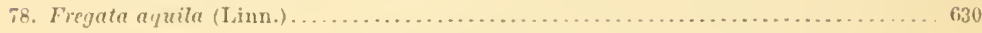

PELECAXIDE.

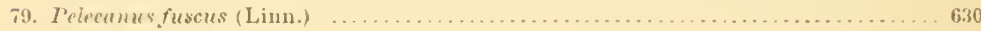

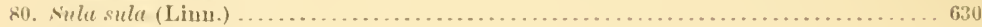

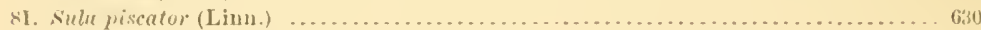

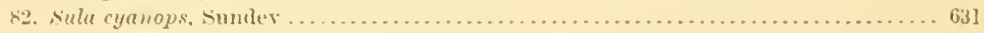

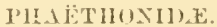

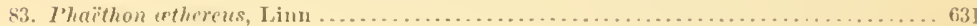

I.A IIID.E.

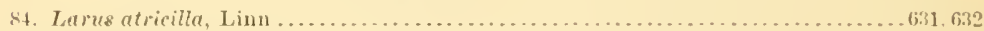

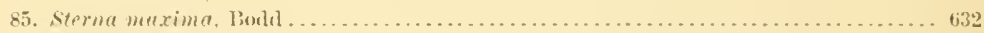

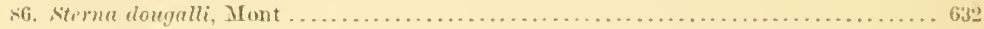

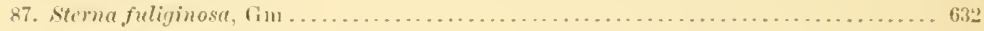

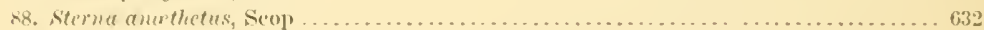

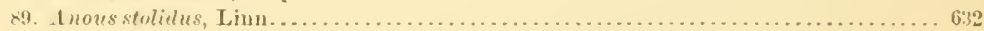

PROPLILARIII)Z.

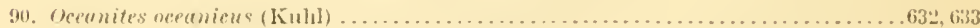

IMHCHPIHE.

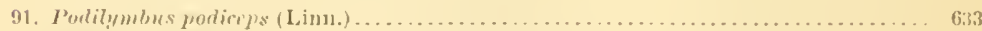

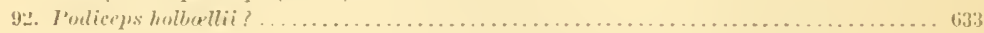

\section{2.}

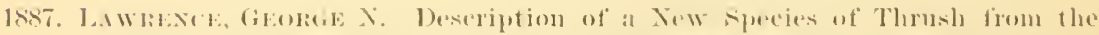

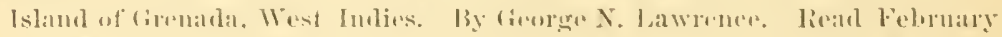

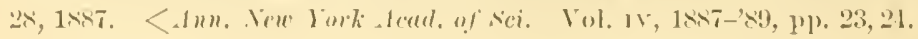

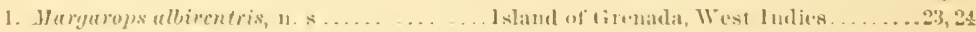

113.

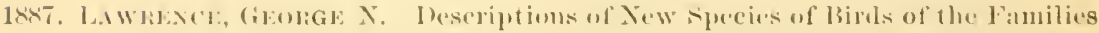

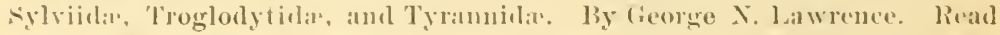

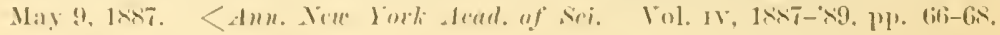

l'iare.

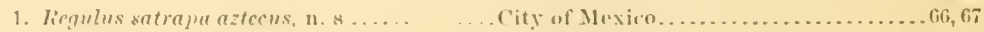

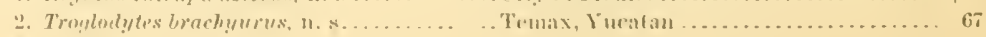

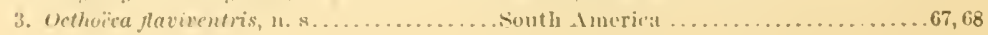


114.

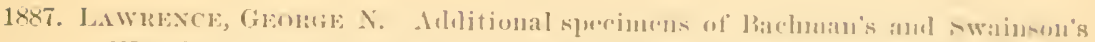

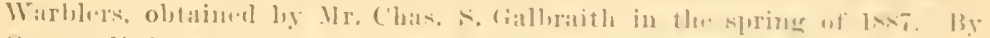

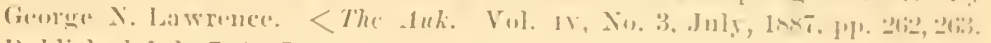
Pnhlished July $7,18 \times$.

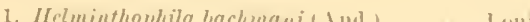

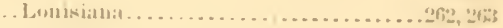

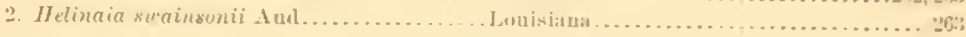

\section{5.}

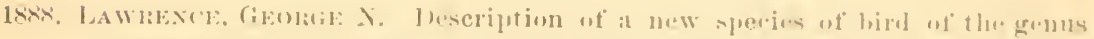

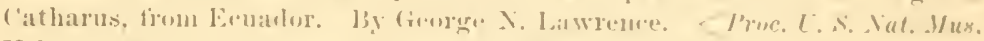

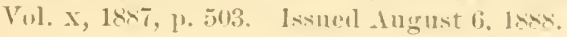

1. (hathariz berlejseni,

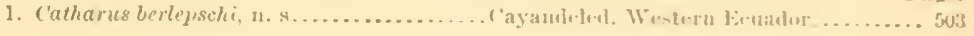

116.

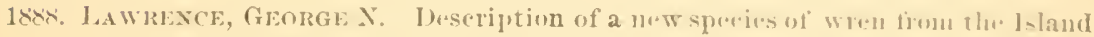

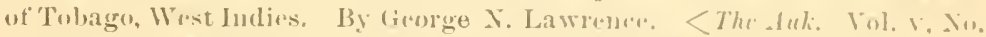
1v, Octuber, 18xx, [1. 404. Published Octols.r 23,1 xis.

P'ag:

\section{7.}

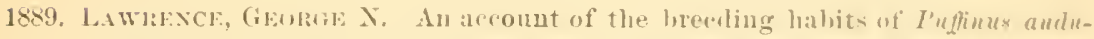

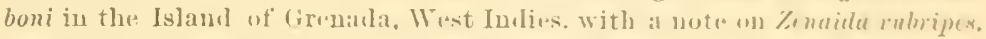

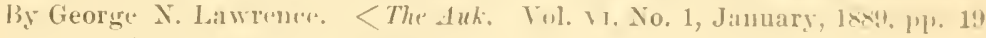
-21. Published Januiry 4, 18x9.

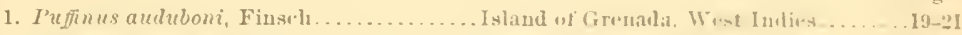

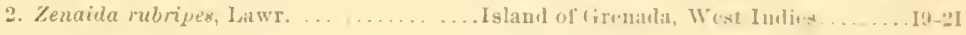

118.

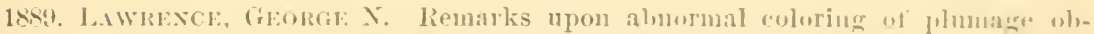

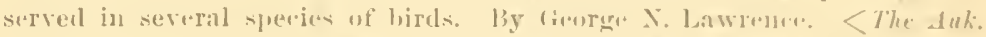

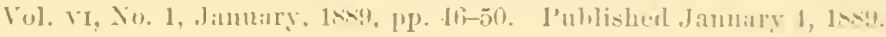

1. Isittorius suber meleus .

2. Vireolanius pulehellus ........................................

3. I'rucuias tersa ..............................................

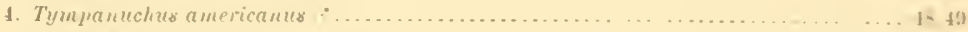

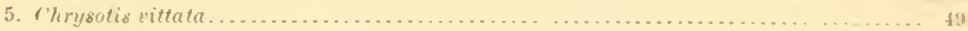

6. C'hrysotis amazonica var. dourestica.............................. 50

119.

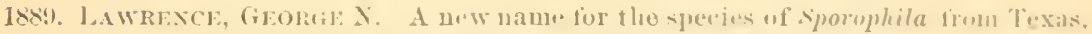

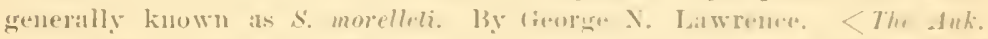

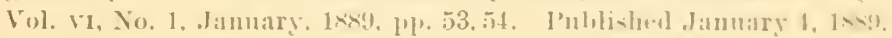

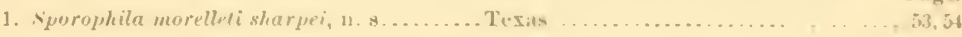

120.

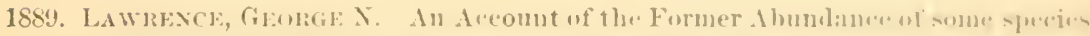
of Bircls on New York Island, at the tine of their Migration to fhe south.

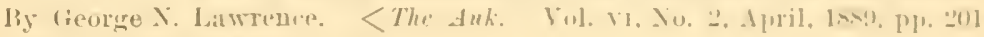
-203. I'ullisherl April 1, 1*\$9.

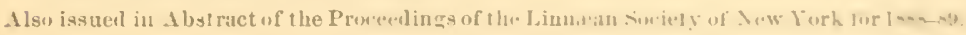

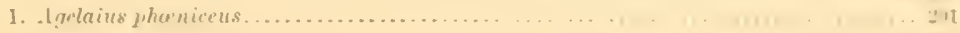

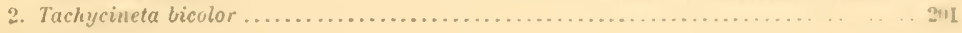




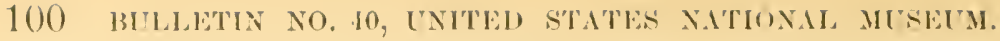

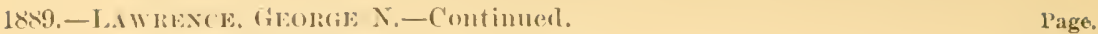

3. rheliton crylhrogaster .......................................... 201

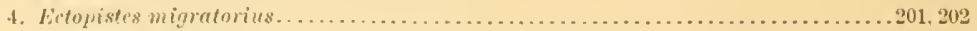

5. т!нанин !

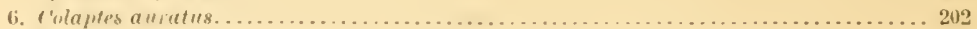

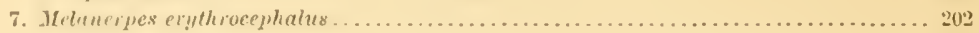

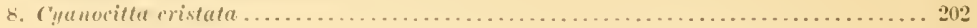

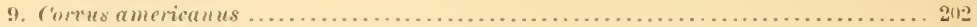

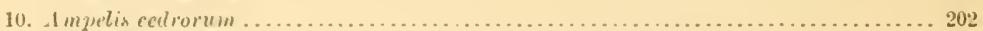

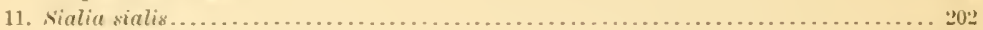

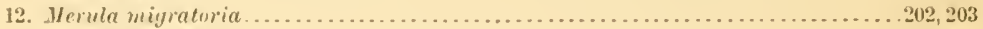

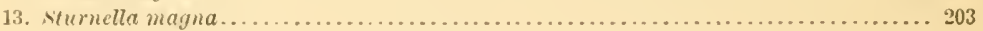

121.

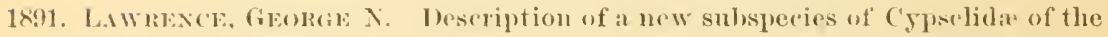
genus ('hotura, with a note on the lialolotin. liy Georese $\mathrm{S}$. Lawrence. The Auk. Vol, Vur, No. 1, Jammary, 1891, pl). 59-62. P'ullished Jannary $15,1 \times 91$.

l'age.

1. ('hatura dominicana colardeni, n. sub s.. Guarlelenpe, West Indie's. $59-61$ 
PUBLISHEL WRITLYS OF GEORGE NEWHOLD LAITENCE, 101

\section{ALPHABETICAL LIST OF NEW SPECIES AND SUBSPECIES OF BIRDS DESCRIBED BY MR. GEORGE N. LAWRENCE, 1846 TO 1891, WITH HABITAT OF TYPE SPECIMEN.}

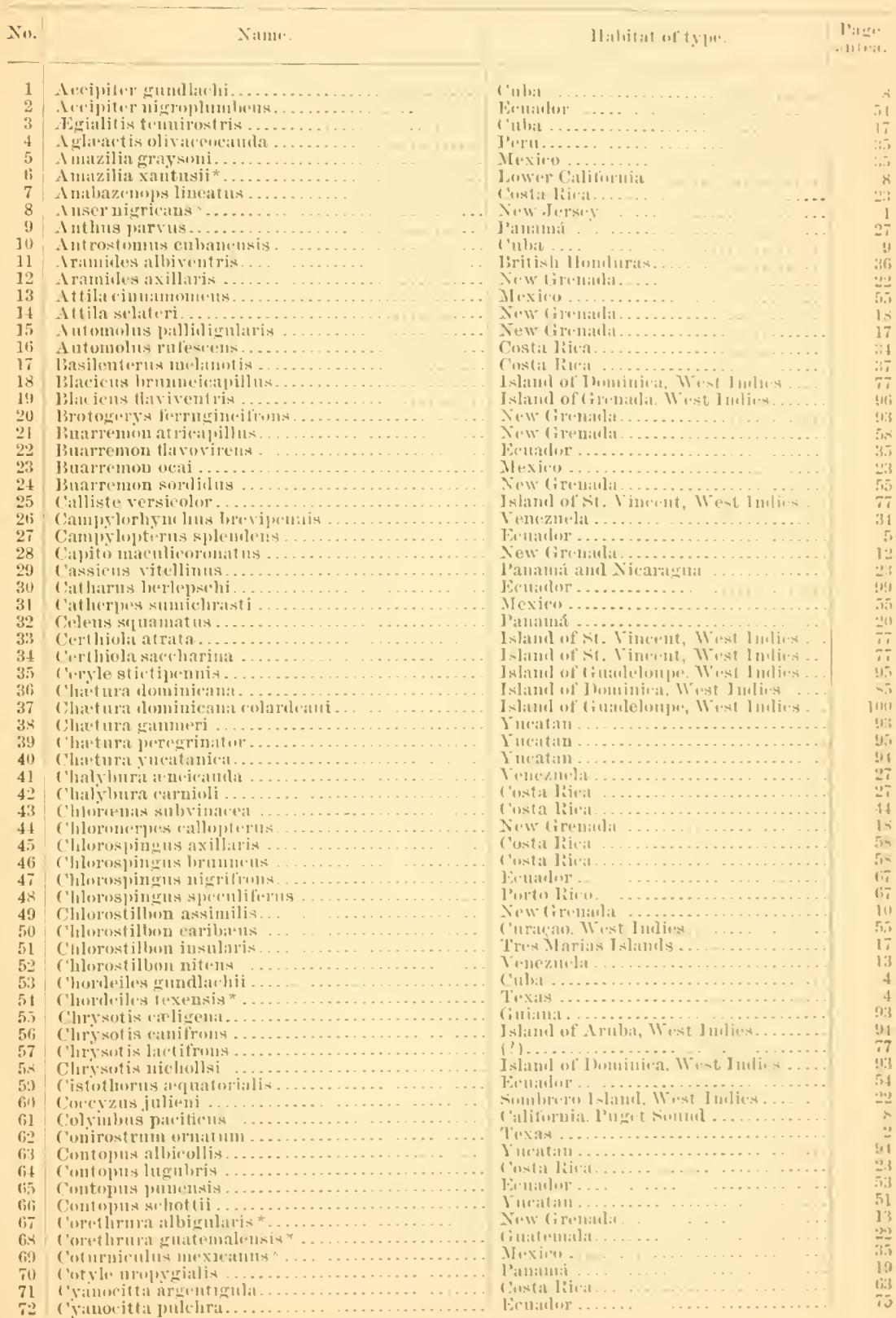




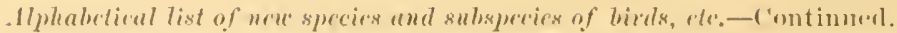

\begin{tabular}{|c|c|c|c|}
\hline Ni. & Xiture. & llabitat of 1 yede. & $\begin{array}{l}\text { I'agn' } \\
\text { anfera. }\end{array}$ \\
\hline$\because 3$ & (yamoenrax ortoni. & l'aru & \\
\hline 7! & 'Yancospiza rosilat. & Mr.xin & \\
\hline 7.7 & 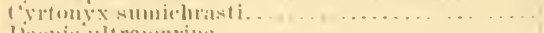 & M(.xilou & i* \\
\hline iti & 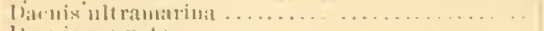 & l':inauná … & \\
\hline 77 & l:acnis vennsta....... & New limenasla. & 17 \\
\hline & lenclura capitalis. & Barlbarlus... & \\
\hline 79) & 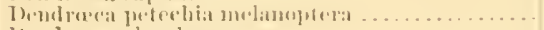 & 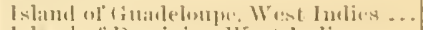 & $\leqslant 9$ \\
\hline$\Delta 1)$ & 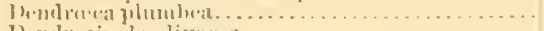 & Islamul of Duminina, Wrest Indies...... & \\
\hline ह'l & 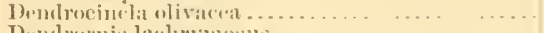 & 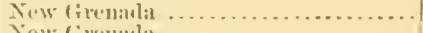 & 17 \\
\hline+3 & Demdromis lan-lirgmesus . . . . . . . . . . . . & New (iremala .............. & \\
\hline 83 & 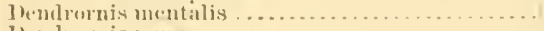 & Mexires ... & 3.7 \\
\hline 84 & J' ndlyoruis natuat ........ & Panamít... & \\
\hline$-i \overline{5}$ & Juriclua bryantal .......................... . & Cinsta licie. & 25 \\
\hline 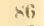 & 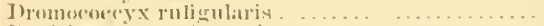 & fiuatemuala. & \\
\hline 87 & 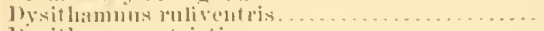 & Now firenat: & 23 \\
\hline sis & livithammos striaticepls . . . . . . . . & (onstia libra. & 23 \\
\hline$\therefore 9$ & Elininea cliringuenesis ... & New liremadil........... & 25 \\
\hline (9) & Elanesea lrantzii ..... & 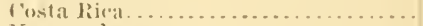 & \\
\hline !1 & $\ldots \ldots \ldots \ldots$ & Vinuezurelil. & $\therefore$ \\
\hline 92 & Elitinea semillasia ... & New Grenatda. & 25 \\
\hline 93 & ratutivirgata* & Texas. & \\
\hline 91 & Empinlonax al rirustrix...... & 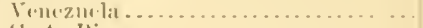 & \\
\hline 9.5 & unis $\|_{\text {a }}$ & ('ostit Jícil. & 23 \\
\hline 96 & Empilonas fiulripertus. & Mpxiro ..... & \\
\hline 97 & $\ldots \ldots \ldots$ & Ne't tirenala... & Is: \\
\hline $9 \mathrm{~s}$ & Empitlonax grisupertns.... & Eruarlur ......... & \\
\hline $9 ! 9$ & $\operatorname{Em}$ & Sanfer I lo & iii \\
\hline 100 & Empinlonax peetomali. & New Girenada............... & \\
\hline 101 & Mit 20 & 10 & !! \\
\hline 1022 & ........... & Yusitan & 95 \\
\hline 103 & n.......... & dil, Wirest Indis's........ & 94 \\
\hline 10.1 & wh.tes oceilentalis .............. & 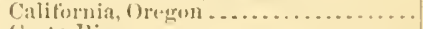 & 233 \\
\hline $10: 5$ & Eup & Cos & $3 i$ \\
\hline $104 i$ & Einjlien & 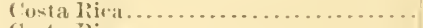 & \\
\hline 107 & il: $a^{*}$. & & 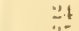 \\
\hline 108 & Eni & $\therefore$ & 335 \\
\hline 109 & & & 91 \\
\hline 110 & For: & 1) & \\
\hline 111 & ulur. & ( ) & 24 \\
\hline 112 & For & l' & \\
\hline $11: 3$ & & & 27 \\
\hline 114 & *. & liea. & \\
\hline 115 & is. & . & 44 \\
\hline 116 & ........ & a liara. & \\
\hline 117 & agmenkis & iacka.. & 35 \\
\hline 118 & t & ta Riv: & \\
\hline 11! & t & $\therefore$ & 5 \\
\hline $1: 1$ & ( & i'a. & \\
\hline 121 & 1 & & 34 \\
\hline $12 *$ & … & ... & 13 \\
\hline 123 & is & Sl Croisanelst. Thonas, Went Inelies. & 77 \\
\hline 121 & I & 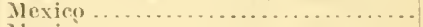 & \\
\hline $12:$ & ichrasti.... & Me: & 54 \\
\hline 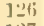 & & (i) & \\
\hline 127 & rummelis... & l': & 16 \\
\hline & & C & $3 \pi$ \\
\hline 129 & Inlilia* & f & 35 \\
\hline 1310 & & & \\
\hline 131 & Hocamla & milia ... & 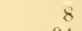 \\
\hline & & 1 $\ldots \ldots \ldots, \ldots, \ldots, \ldots$ & 94 \\
\hline $13: 3$ & il & .. & 3.5 \\
\hline & & & 27 \\
\hline 13. & H & & 14 \\
\hline & & & 14 \\
\hline $1: 37$ & liav & la.... & 14 \\
\hline & Ic & & \\
\hline $1: 2$ & 1.1 & & 5 \\
\hline & & Wrot luilies. & 93 \\
\hline 11 & $\operatorname{lom}$ & 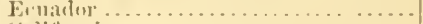 & \\
\hline & & & \\
\hline It: & kleyi. & ... & \\
\hline & $\mathrm{L} \cdot \mathrm{l} \cdot \mathrm{L}$ & & 15. \\
\hline 14 & rtii . & Mexion & $5:$ \\
\hline & & ............. & \\
\hline 1.17 & Leploptila fulviventris" & . $\ldots \ldots \ldots \ldots \ldots \ldots$ & 94 \\
\hline & & & 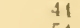 \\
\hline 1.1 & 1. shbia a & $\ldots, \ldots \ldots \ldots$ & 5 \\
\hline & & Islamel of st. Vinernt, West Imlies.... & 7 \\
\hline li. & Linevplernis ke & 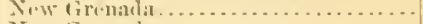 & \\
\hline & 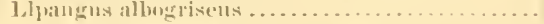 & New Gronata.. & \\
\hline
\end{tabular}




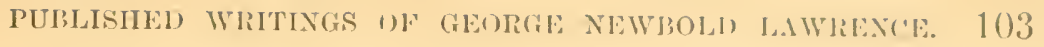

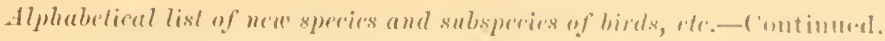

No. Nime.

ii Moxigilla portoriandsis groudis

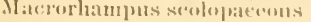

Margarops alluseutio . ....

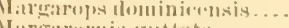

II argaroruis gutlata ......

II

Ardlisuga albo-(1)ullatal.

I[r]lisugit morrittii......

Mr.lopelia phomluestens.

Minus molanoptrins

Mimus nirriloris. . . . . . .

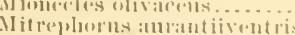

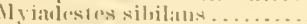

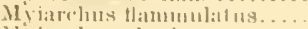

Mlyiarelies utwri

Myiarchus panamengis

Myiarelats selatteri.

Myiarehus vemezurensis

M jiarrlate volutanensis

Myiobius atric:and

MÝndymates smpereiliaris

IJjometoles gramalemsis

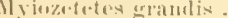

Ifiometetes inornatus

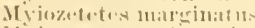

Mjozelotes rutijuluis

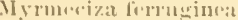

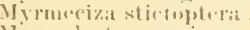

Mlimulasteseorvinus...

3) yrumblerula alliguta

Iyrmotlerula molesta

(hetherie:at thatsiverutris.

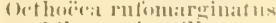

lochilus atricapillus.

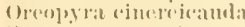

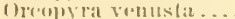

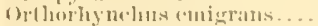

()rt $y x \leq x: y$ : $2011 \mathrm{i}$

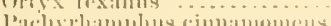

l'anylhlora joirvirostrise.

l'arilat insularis..

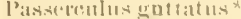

Pry romelelinlon allilimea

l'lictlomenis at rimontialis

l'lathlurnis masinii

phathormis momrei.

l'hat lonmis nieriointius

P'hart lommis villonms.

ghatom flavo-aullautius

ihemeticus tibialis.

l'hilyclor rutolnomuetis .

P'lilvilur vireatess.

l'hunicothrampis atamioli

l'hreniesthanupe eristat al

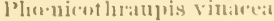

l'houipatrat limoxa.

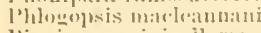

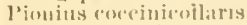

l'ipilo ratmani

l'ipral ejunantume:al

l'itamens alboviftatus

l'it:angus qubliit.... . .

l'illıs Lirolur.......

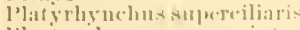

l'lerfropliames mecownit

l'uliveys relarkii

l'orlicrjos onecislemtalis

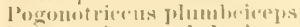

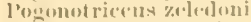

lolioptila alhiventris.

Inolioptila numanurat .

lolioptila plumbicepen.

l'olioptila suynereilitrris.

porzantat rimere+iceples

P'rocellarda brevirostris *....... llabital of ti le*.

I'agen ante?.

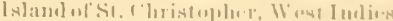

lalli.y, aml wial waril

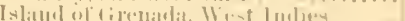

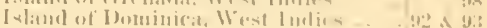

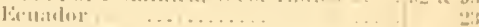

liustit livirit ... ...... . .

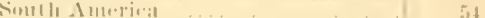

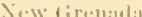

Yin limenalit

tillidail

1.12120\%11101.1

Mrxion?

fintil linial.

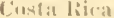

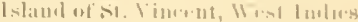

Misican

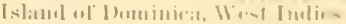

I'allamili

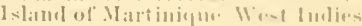

V'"114"\%1u+lat

İu:atin

T'animition

fostal lier:a

T'allimil.

I,

bincku1, lit.

I'anami

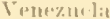

Xiw timenamia

t'ustat Iinc:at

Jiew Iirrenierlit.

New limmatal.

New tirinatilit

l'ostat liciol

Sonth 1 interirit.

Fillarlol".

('on)tia lióical

('ostia líio:al.

Contaliant

Sent?mala

Mexion

Texily

Vew finendula.

fostar livein.

Tres Marian Injomi

Lomere tialiturniat

New lirellada.

Penariur.

New limenalit.

Eirarar

Eentarlor

Finatur

t?

firsia Iticis.

Contil libira

fosta licial.

fistat licicat.

$\mathrm{New}$ limoulal

Sew limenarla

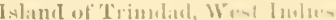

I'amaluát

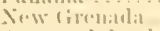

Corepra lsland

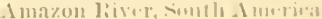

I':เ1กเแลi

Situto Dumill:-u

Xis limenallit.

l'an:m1!i .....

Tresils

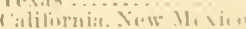

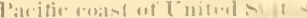

Viw limellimal...

C'ostal liviol

Yuritiull

levas raliliorulit

Ionevireln

Xiv I r r niulat.

losta likieral

Florida

llix livt. 


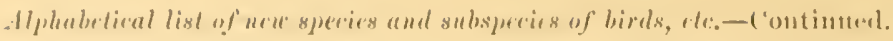

No.
Nimu!

Irowellatia mordionalis

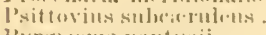

l'yrrmsmal xilltusii .

l'yrrhoplen na graysuni

pitilus humeralis.

Giinsoalus fortiresstris

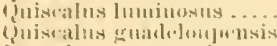

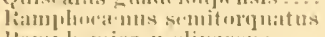

liamplumicrul alivacetis.

licgulus sat rapia azterus.

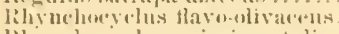

lihineluegelus grise-imentalis.

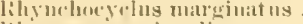

liksis sertiontrionalis.

Saltater intermudius .

Sialtator julumbiceps.

Silphirmial luminosil

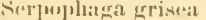

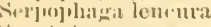

sieotyto amamra.

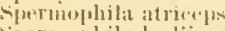

Spermophilat baliivantris .

finemenephila collatris

spermojolila fortipes

Sperounguilat licksii.

spernephlifa parsa.

Fpermophilit sebistacea

spermophilia semicollaris

sporatinus luacei.

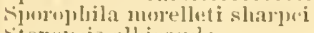

stenuplis allitaudia.

it (tur) is maculicautus.

Sterna pikei.

trix llammeta nigrescens.

Gymallaxis maculata...

Fymallaxis migrifumusa

fynallax is rufigenis.

รyтnimm line:t 111 .

Tacliyplumus at ricalpillus.

Taclyyjumts rassinii..

'fachiphomos napensis

Talchyphomus propingutis...

lacliyphonus rubritrons .

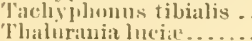

Thammophilus loblenuli

Tlrammophilus leweopygus

Thammophilus nigresentns.

Tlammophilus temuibiseiatus .

Thammophilus virgatus..

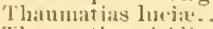

Tlimumat ias viridieamilu.

T'uriparlestes virgaticeps.

Thryothoris hrumens.

Thurothorus galbraithii.

thryotluorus gremanlensis

Thriot lourus musiens.

Thervothoriss rufescens.

Throwt lom supereriliaris.

Tinaums trantzii...

T'mlirostrmu olivas"

Fodirostrtm smeereiliaris.

Toxostemal leconter
Troshilus antigntaris

Truglowlyas hatehyous

Tomilawlytes folnotginsis

loment litiralii

Trogen ancintums.

Tromon eximits.

Turdus brinnells

Turdus cariblad ins

Tumlus niegrirostris

Timilus alisulatus.

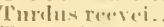

Tyrauniseus parvus... llabitat of tyle.

Florida

1

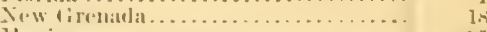

Mexiro .......................... is

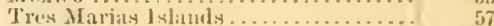

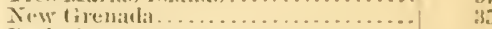

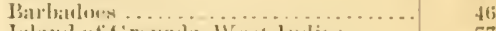

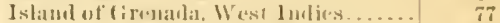

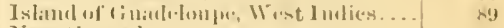

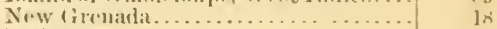

Ibliviat ......................

Inxi(c) ...........................

Nirw tirenalla...................

Contal licici................... \$11

Jinnimí........................ 47

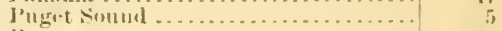

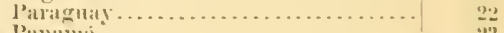

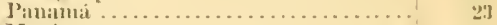

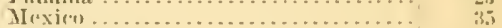

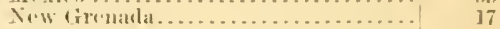

Custat likil ...................... 5.5

Eintuklor....................... 67

Islaud of dittigua, West Inties .....

Iexiro ...........................

Niciragua ................. 2f

New Gruarla..................

Niw Grentala ...................

l'allimí ........................

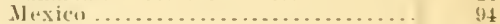

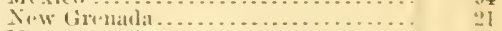

Jew tirenala ...................

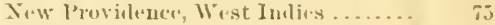

Texils ...........................

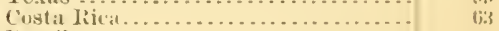

13ra»il ............................ 17

California .....................

Island of Domizira, West Indles......

l'er'll............................ 5j

Nicitragna ................... 26

fosta firit .....................

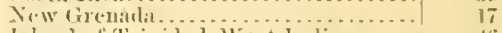

Istand of Trindad. West Indies.....

Vew dirmadia...................... 12

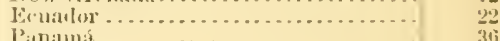

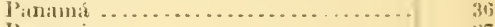

P'antulit ...

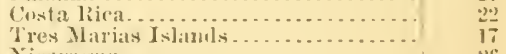

Niratugu: .......................

New lim nadia................... 35

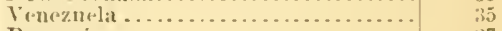

Pannmi .......................... 27

Eollader -

$X_{1} w$ Iirmala................... 46

Honduris.....................

Niw Grenama.................. 35

Beuader ... is

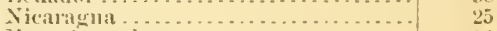

Niv (imbula................... 14

New lirrmilia.................... 14

Island we Cirenada. Wrest ludir.......

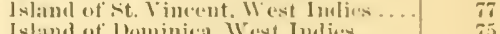

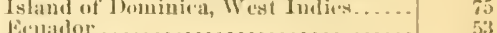

Costat licia.............................

l'amamí .........................

Vimeguelit .............................

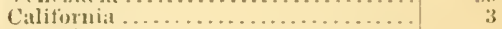

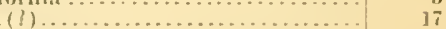

Vintallan

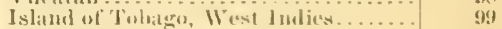

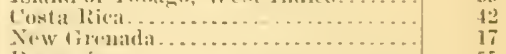

l'an:min ........................... 55

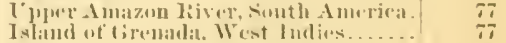

Irru......................... 51

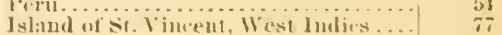

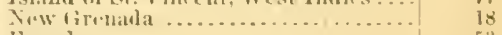

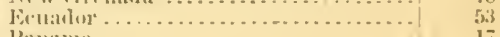

l'mulut ...................... 17 


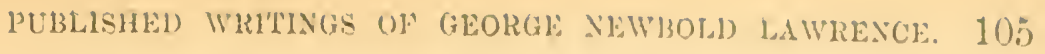

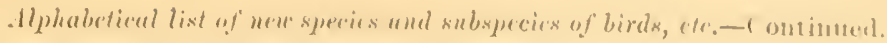

\begin{tabular}{|c|c|c|c|}
\hline No. & X:mu. & balutat of tylu" & $\begin{array}{l}\text { l'as.". } \\
\text { anle:il }\end{array}$ \\
\hline & 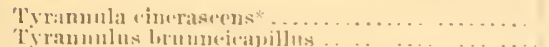 & $\%$ & \\
\hline & assiniti... & $\begin{array}{l}\text { Pan:mua } \\
\text { Yoxis: }\end{array}$ & \\
\hline & & & \\
\hline & miv. & 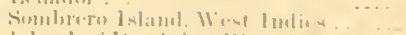 & \\
\hline & li & i lluit & \\
\hline 2 & Xil & 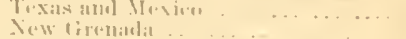 & \\
\hline $3 \div 2$ & Ye & 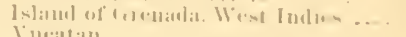 & \\
\hline & Zonotriclitil melanotis* & & \\
\hline
\end{tabular}

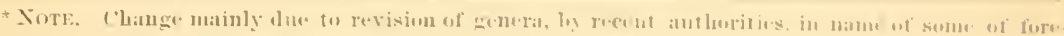
going new species and present stitus of sume ot leers:

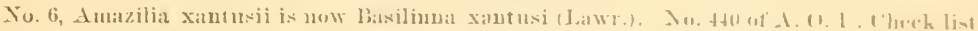

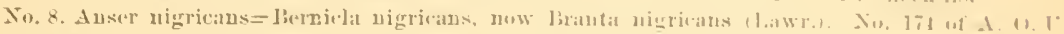
C'heck.list.

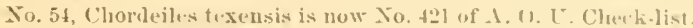

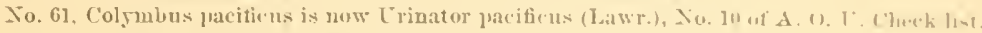

No. 67, ('oretlarural alloigularis-l'orzana albignlaris.

No. 68, Corethrura guatemalensis likewise was placed in the grans I'or\%and

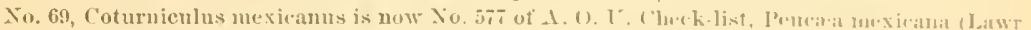

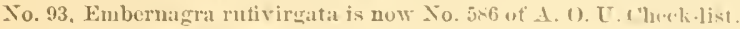

No. 104, Eremuctes orecileutalis is now Xo. 24 of . 1. 1). U. ('berk list.

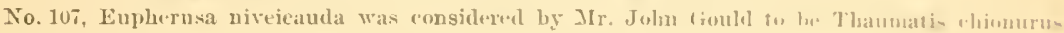
Gould.

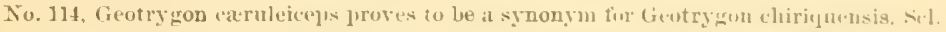

Xo. 129, Helioulaster spectabilis was afterwards plated in the genus buenes.

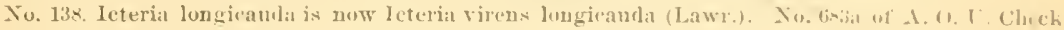
list.

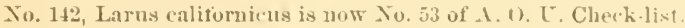

Xo. 147, Leptoptila fulviventris= Engyptila fulviventris (Latwr.).

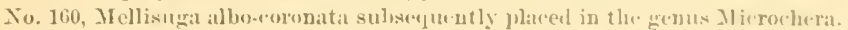

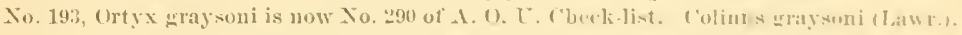

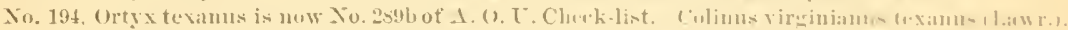

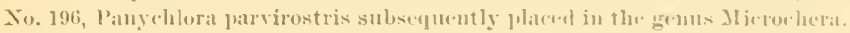

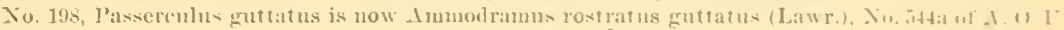
Check-list.

To. 199, Petroeluelidon alhilinea:= Tachyeineta albilin:a.

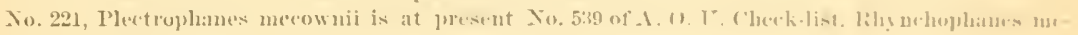
comnii (Liwr.).

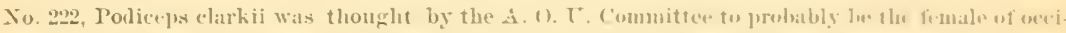

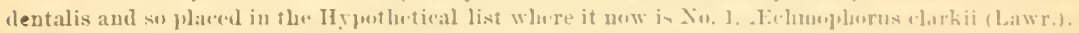

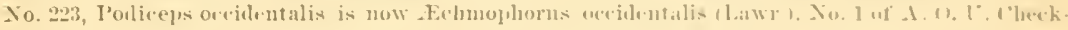
list.

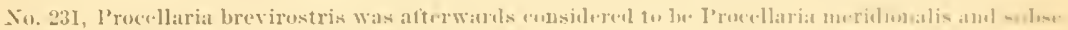
quently called . Estrelatil meridionalis.

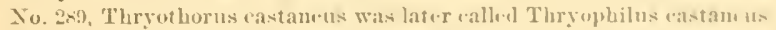

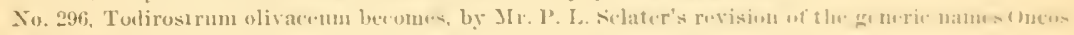
toma olivateat (Lawr.).

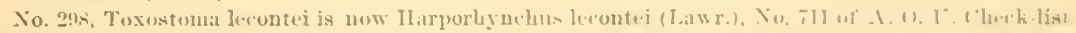

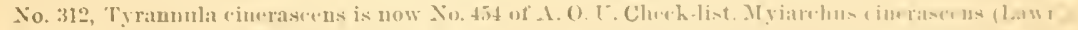

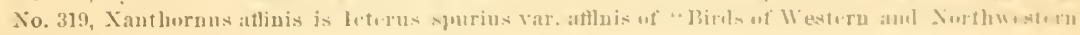
Mexiro."

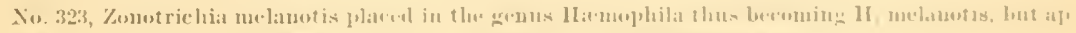
pears as Aimoplhila melanotio in " Birels of Westeru aud North western Mexico." 



\section{АLPШАВETIOAI, INDEX.}

A.

Academy of Natural Scionces, P'liladel-

phia, l'romedinger ol ......x, 22, 23,27,36,46, 55

Aecipiter collaris .................. 17 cooperii ..............2 $2,4,47,63,72$ friugilloidey ............... 9 func'las. .............2, $44,47,6.3,72$ guthlahii ................. ^. 101 nigruplumbens............ 54. 101 pileatus ................. 9,44

Aetitis matularia .................

Actiturus bart ramius ................ 16, 32, 45

Artodromas bonapartii.............. 32

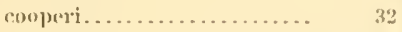

maculata.............21,32,73,977

uiuutilla................ $32,64,73$

Echmophorns markii............... 105 oreid.ntalis............ 105

Egialitivayaro ................... 16

cullaris...................

metodus .................. 32

nivoงแแ ................. 5.3,64

semipistunta $\ldots \ldots \ldots \ldots \ldots \ldots \ldots .22,32,54$

$57,61,73,81,81,90,92,97$

temirostris ............... 17, 101

vocifera ........ $19,32,45,50,53,64,73$ wilsonius . . . . . . . . .

Eqiotlın linaria ................... 30,89

Estrelata merillionalis ............... 34, 105 spl. ! …......... 92

Agelatus gubernatur................. 61

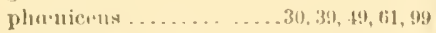

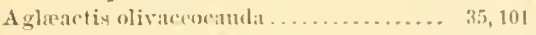

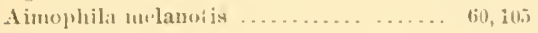

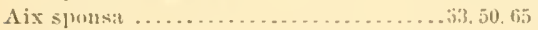

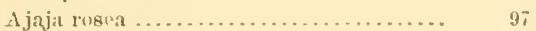

Alaskil .......................

Alanda arvensis .................... 49

A laurlidlie ........................30, 49, 61, 69

Alcesliuide ...................... 10,1:2,26.

$31,41,47,52,57,62,70,79,81,813,87^{2}, 89,97$

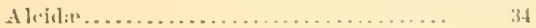

Allen, \#r. J. A ..................... xi

A manrospiza rourolor................. 39

Amazilı: dum rili ................... $5 \downarrow$

gritysuni ...................... 35,101

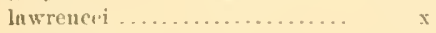

riefleri ................... 10

xantusii .................., 101, 10,5

Amเazm River ..................... 17,$7 ;$

Amblyrerons preronti............... $\quad 39$
Ameriona Musenm of Natural Iistury

Bulletin wf .................. xi

Americ:u Nitturalist, The............ \%

Amerian (Mmithelogiats' Inion........ viii

Ammoraranus (Ammolromus) calldal to maritimus. ............. 30 rostratuy gutt:atun ....... 111;

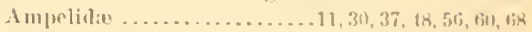

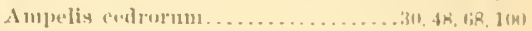
garrulus .............. :

Anabates cervinigularis ............. ochrolismun ................ 11

A nahatilla........................ 11.14

A nabuzenopes linmatus ................2:39. 311 varingatiorelw.......... 39

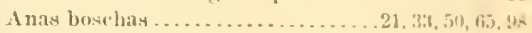
discor. .........................

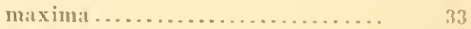

obselura ....................... 33, tis penelopen.................... 1

Anatidid ..........21, 33, 46, 59, 53, 65, 74, 83, 44, 4.

Aurylocheilus suharenatit ............ 32

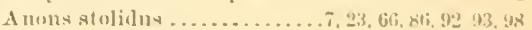

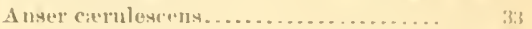
gambeli ..................... 33, bi.

byperloren. ................. 33

nigrio:aw .................. 101, 10.;

Antenor unitinctus harrisi ............

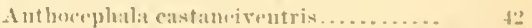

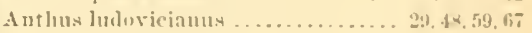

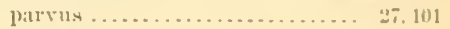
rufus....................... It

Antigna. Inlatul of...................2, 83, 101

Antillew, I.

Ant rostumus carolian.n. cub:unenvis ........... 9. 101

rorifirms................. $31,7,71$

A pluriza virgata................... of

Aquila canadensis ..................

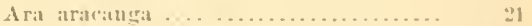

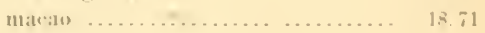

militaris....................... $1: 1$

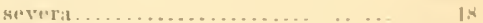

Aramides allivintris ...... _.... . at, it 101 axillaris . .............. .

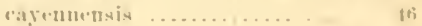
rutioullis ................ 1!1, 25

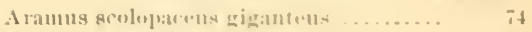

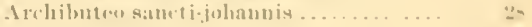

Arutir Amerira ..................... fi. $\mathrm{i}$, h geas $\ldots \ldots \ldots \ldots \ldots \ldots \ldots \ldots \ldots$ bi,, i. 
Page.

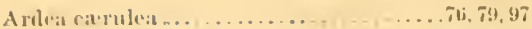

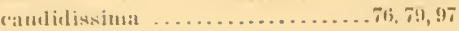

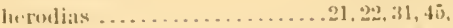

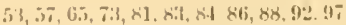

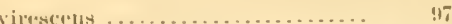

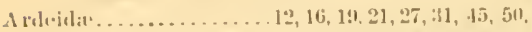
$533,57,58,65,73,79,81.83,84,86,86,90,47$

Ardeltacilis..................21, 32, 50,65, 7:

Ari\%min

Arinatellat maritima.................

Arremon aurantiirostris . ............. $12,26.3 \mathrm{~s}$ ruticlorsallis.................. it

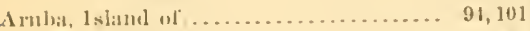

Astur at rimpillus.................... 2n

Asturinat magnirost ris .............13, $25,4.4,52$ nitillit..................... $13,44,46$ plitgiat:1.................. $16,63,72$

thene cunicularia................. 56

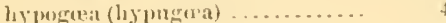

Allititic Geran .......................

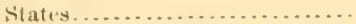

Atticora ryanoleucit unomtanat ........... 37

Atthis helena.......................

Attilit cinnamome $u$. .............. 55,61, 101 citreulygins ................. 21,70

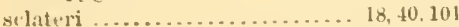
sparlicea ................... 15

Auk, The................. ix, x,94,95,99.100 Anlacorhamplus carmleigularis ....... tit

Auloumelus cervinigularis. .............. 39 palliligularis ........... 17,39, 101 rufiseris................. 34,39, 101

Asthya amerirama ................. 33,65 vallisueria .................. 33,65

B.

Ballins liay

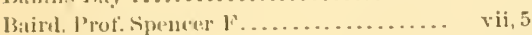

Barlo:ulos loliand .................. $46 \quad 102,104$

Barbudia, 1slamel of . ................ 82, 83, 84

Bartramia lemgicatuda................ $9 \overline{9}$

Bisilenturus culcivorus.............. 37

delattri................. 14

melamenenys............. 37

nutlanetis................ 37,101

mexulhrsus ............. 37

rutitruns............... 59,68

semilervinus .............. 14

uropygialis ............. 25,37

Basilinna xantusi .....................

Berings st raits.....................

B.11. Jolut.

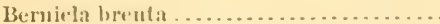

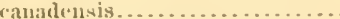

camadensis oredulentalis .........

hutrltumii ..................

honcoumin.

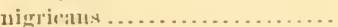

Biologia Contrali-Anericilust .............

Birds of Lomg lslimel.

Biselun liverd

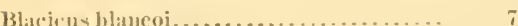

1,runure:apillus ........ $77.78,911,95,101$ 11:15 ivent ris................. 91, 101

Blasipux heermam
Bulivia......................... 22, 104

Bonap)arte. ( $.1 . \ldots \ldots \ldots \ldots \ldots \ldots \ldots \ldots$ ix

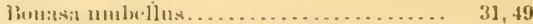

Buston Soredy of Natural llistory-

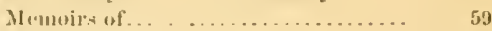

Proserelings at' ... . . . . . . . . . . . .

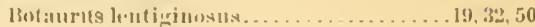

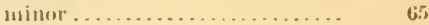

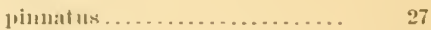

Mrathyalbat lugubris................. 54

Brachyotus asssinii................. 2x, 47

Brachypturi....................... \&

Brantat lentopsis.................. 55

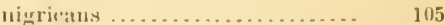

Brazil ..................... . . 17, 55, 71, 104

[3ritislı liuiaua.................... 94, 102

I3ritimh (3rnitlologists' [nimn .......... vii

Brotugerys (Brotogeris) forrugineitrums. 93,101

tovi .......... 43,71

Busremon assimilis ................. 38

atris'apillus.............. 58,101

brume.inuchus............ 38

Mrysopmgon ................ 38

crassirustris.............. 38

Havovirens............... 35,101

mesoxinthus ............. 38

weat $\ldots . . \ldots \ldots \ldots \ldots \ldots . .23,101$

sorvililus .................. . 5,501

Bubo virginiaums (virginianit)....28, 44,47,52, 72

Buceodysmi ....................... 13,

pectoralis .................... 17

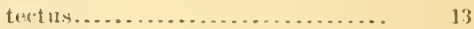

Bucconilia...............10, 13, 17. 20, 26, 42, 70

Bueplata allwola.................. 33,50

americima ................... 33,65

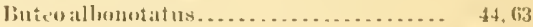

lmrralis.........................

lorealis valurns .............. 63

burealis rostarienusis............ 63

borealis montanus............ 14,52,56, 5R

prythronutus ................ 44.52

finliginısus .................. 44

gluishroghtii ................... 9

harlani ...................... 3

litissimus .................. 97

linuttus ................... 28,47

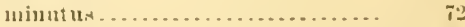

nigri(collis .................

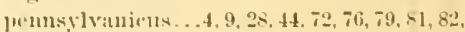

$86,87,91$

piteroulos................... 72

loutorilles brinumese'ns ................

viresce-11s . . . . 13. 32. 45 50, (65. 73, 76, 79

s1, $83,84,86,8 \bar{i}, x+4,90,92$

C.

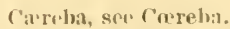

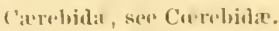

Cairint umest hat a ....................21, 65, it

Caliuris arentria...............32, 53, 64. $73,81,92$

Gillilorniat ......... ix, $3,4,5,6,7,8,101,102,104$ 1,1 w.r............ $7,8,35,101.102,103$ sontlu.r. ................ $\quad$ i,

Callipulil clegany ................... 64

sepramata.............. 4,64 
Calliste dowii $\quad$ Pils:

frincesew ........ . 12, 16, $24,20,38$

frantzii .................

guttatil...................... is

gry roloides .................. 15. 38

ieteroceplisla ................

inuruatit.................. 12

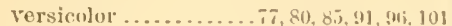

Callocitta colliei ................... (i]

formosa ...............

Calothurax lucifer................... b. iti

Camponluilus guatemalensis...........43, 1i2, 71

Cimptolaimus (Camptolaemus) labrado.

rins ......................

Camptostoma (Camptosoma) imberbe .... 1s.is. 61

Campylopterus cuvieri .............. It

hemilencurus.......... $\$ 2,7$

splendens............. 5, 101

Campylorhynchus breripunis.......... it, 101

eapistratus ........ 36

guttatus........... 50

humilis............ 59.67

jocosus ............ 67

zonatus ............ 36,67

Cancroma coclulearia . .............. 19,45, 64, 73

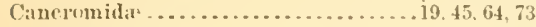

Capito bourcieri.....................

hartlaubi ................. 43

uaculicoronatus.............. 12, 101

Capitonid:e ...................... 12,43

Caprimulgilla $\ldots \ldots \ldots \ldots 9,17,31,42,47,52,56,62,71$

Cardinalis sinuatus ................. 2

virginianus................30,51,57

virginianus carnens .......... $.60,69$

virginianus igneus.......... 60

Carduelis lawrencei.................. ix

Carolina .......................... i.

Carpotacus frontalis................ 60

purpurens................ 30,49

Carpolectes nitidux............... 26,41

Caryothraustes humeralis.............. $\quad 35$

Cassiculus ictcronotus ............... 11

melanictırus .............

microrhynchus ...........

provosti.................. 11,20

uropygialis................ 11

Саянісия prewsti ................... 69

vitellinus.................. 23,102

Сам⿻і㇒lix haritus .................. 11

erassirostris................ 11

Cassin, John ..................... $1 \mathrm{x}, 5$

Cathartes utratus................ 20.63

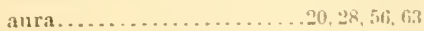

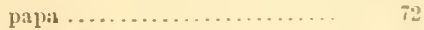

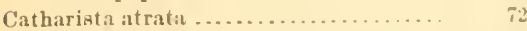

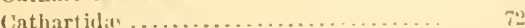

Catharus berlepschi................. (9! 101

frantzii ..................... in

fuscater..................... in

gritcilirostris.............. 30

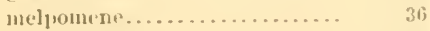

mexicamus .................

occidentalis ................. it

Catherpes mexieanus .............. 59

suluiclirasti.............. 5i. 101
$27,+3$

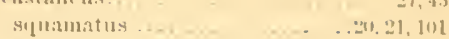

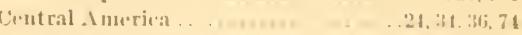

Ceut ral l'ark, Niw Jork ('ity w....... ti, il)

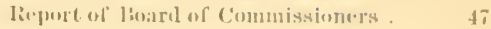

(inuturus alhifrums........... . . . 5

andifruns......... $\quad$ il

riruliuus .............. 31

rlegrins.................. $2,6,2$

gerini ................ 43

lothmani................. 41

hурищйіus................. il

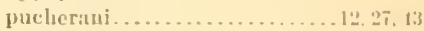

rubriventrin . .............12,52.94

santiz-cruzi ................ a

uropygialis ............... fis

Cephalopherus plabrioblis.......... . 41

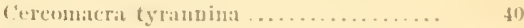

('erthiir ameritanil................ 29,43

Certhialie, sevo certhiitlit.

Certhiislat ...................... 10, 29,49

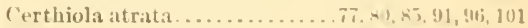
lominicaua ........... $71,78,-2,-3, \times 1,91$ lutcolis .................... 10, 21 martinicana ............... mexicana ................. 3 ; saccharina ............77, 81, 91,96, 101 ap. ?...................... ni

Ceryle alcyon ......................13,31, 11 .

$4 \overline{7}, 5 \bar{i}, 62,70,76,79,81,96, \pi 9,-9,41,47$

amazona ................11, $\$ 1,620$

autricana..................... 210

americana cabanisi............. 62,70

cabanisi.................... \$1

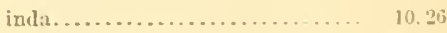

stietipennis ..................... 95101

superciliosa .................10, 41, 52, 70

torquata .............10.41,63,70. 89.91

Chrturat cinereirentris............... 97

dominicana................... 91. 1111

dominicana colardeami......... 100101

g:aumeri ....................93,95, 101

pelasgia ................. 31,47

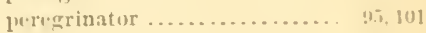

poliura.................. iti. is

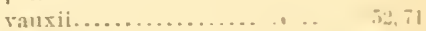

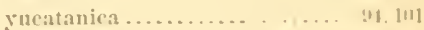

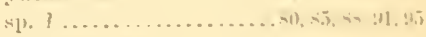

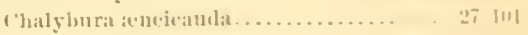

earni li.............. $27,1 \geq, 101$

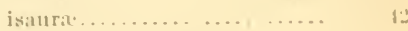

mulaurlina .............

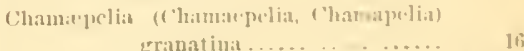

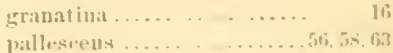

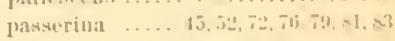

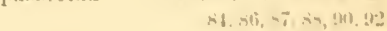

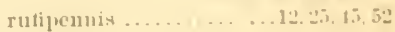

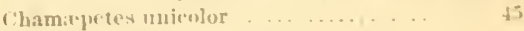

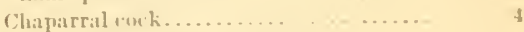

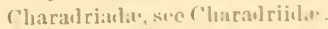

(')aratrisla, seo Charalriidil:

Charatriidir .......16, 17, 19, 32, 45, 511, 53, is , st 64 $73,79,-1,83,-1,-6,90,45$

('haraulrius tominieus.............. vi 
Charalrius sụuatarolis. virninieus.

P'age.

97 $8,50,76,79,8$ $83,84,86,90,92,93$

Chasmorlyoue tricarumculat

Chaulwasmus streperus .............. 33, 650

C'heliclon erythrogaster ............... 100

('hiac kit-lata.......................

Chiromalueris andei .................

Chiroxiphia taudata ................. cyancociptilla ..............

lincaris ................... mutalis ................... vitellinal .................

Chlerewas albiliuea ................. flitvirostris ............... uisrirostris .................

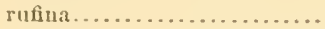
subrinacea............... vinavea ..................

Chlorolampis camaseti . ................ salvini ..................

C'hloronerpes ie rurinosus ............... callouotus................ callopterus ............... oleaginus ............... nropygialis .............. yueutanensis............

Chlorophanes atricapilla.............. spiza.................. spiza guatemalensis .......

Chlorophonia callophrys.............. occipitalis .

Chlorospungus albitemporalis

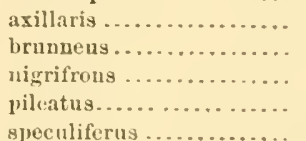
speculiferus .......... $6 \pi, 101$

Chlorostilbon assimilis ................ 10, 43, 101 caribeus ............. 55, 101 insularis...........17,57,62, 101 nitens............... 13, 101 81. ?.................. 14

Chondestes grammaca .............. 60, 69

Chordeiley brasilianu.s ............... 2,42 gundlacliii ............... $\$, 101$ minor.

9 popetue.................9, 31, 47 texensi. .......4,42,52,62, $71,101,105$

Chroicoceplualus at ricilla..........6, 23, 34, 66, 74 rucullatus........... 6 tranklinii........... 6,60 minutus........... philadelphia $\ldots \ldots \ldots \ldots 6,34,60$

Chrysumitris bryantii................ 39 columbiana .............. 39 mexicanus (mexicana) .....16, 39, 57 notita................ 6 ? pinus .................. 30 pyaltria mexicana......... 60 tristis ................. $30,4 !$

Chrymotis alhifrons................4.,52, 63, 71 amaznica domestica......... 99 august a .................... .76, 79,91 auripalliatal.............. 46.71
Chrynt in antmumalis............... Thage.

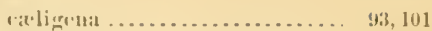

ฯatuifrons.................. 91, 101

farinosit.....................

filısthil.................. 63,71

guatemal: ................ 40

guildin ${ }^{1}$................... 81,91

lut tif rous................... 77,101

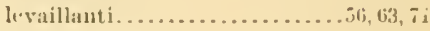

nichollsi................ 93, 101

julverulentil ............... 43

viriligemaliy .............. 4.3

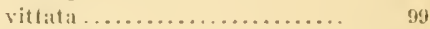

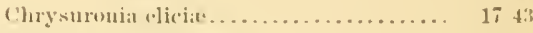

Ciecaba nierolintata............... 44,72

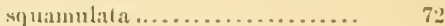

('inclislia ........................

Cinclocerthia gutturalis.............. 87,90 ruficuul: $\ldots \ldots \ldots .70,78,80,83,89.90$

('inclus arelesiacus .................. 30

Circe derubledayi.................... $i 1$

latirost ris................... $5 \bar{i}, 6_{2}^{2}$

Cirens cyaneus hudsonius........... 63, 72

hudsmicus...................13, 28.44

Cissolopla sanblasiamus.............. 61

Cistothorus equatorialis............. 54, 101

palustris .................... 23

palustris palndicola. ........ 59

stellaris............... 29

Clangulit glaucion................. 84, 92

Coceyqus americanus...............31,43,4i, 62 (rythrophthalmus.........19,31, 43,47 julieni................... 22, 101 mìur .76, 79, 81, 82, 81, 86, 87, 88, 90,91,97

r'ereba carncipes...................10, 13, 24

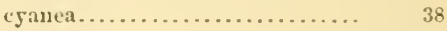

lucila $\ldots \ldots \ldots \ldots \ldots \ldots \ldots \ldots . \quad 10,13$

Ceretridie.............. 10, 13, 17, 23, 25, 37, 78, $80,82,83,85,87.89,96$

Colaptes auratus.................... 31, 47, 100

Colinus graysoni................... 105

virginianus texamus ........... 105

Collurio (Colly rio) horealis ........... 30,48 lulovicianus excubi. toroides .......... 60,68

Colombia........... $x, 4,6,8,9,13,16,17,19,20$, $22,24,27,35,36,46,54,55,58,66$, $93,91,95,101,102,103,104,105$

Columlut corensis........76, $79,81,86,88,90.92,97$

fasciata ................... 63

1lavirostris.............., 4, 56, 63, 72

lexcoes phala ................ $83,81,92$

lencoptera ................

Columlia Tiver..................... 8

('olumlijlite .....12, 16, 19, 21. 25, 31, 41, 49, 52. 56, 5x́,

$72,79.81,83,84,86,88,90,94,95,97$

Cohmubizallina passcrina ............. 97

Colymbidits ................... $8,13,16,34,50,63$

Colymbus areticus................... 8,34

paciticus................, 101, 105

suptent rionalis.............. 8,34

torquatus ................ 8, 34,50

Conimotrum ornatm................. 2, 4.101

(')

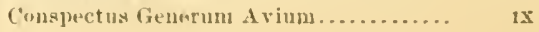




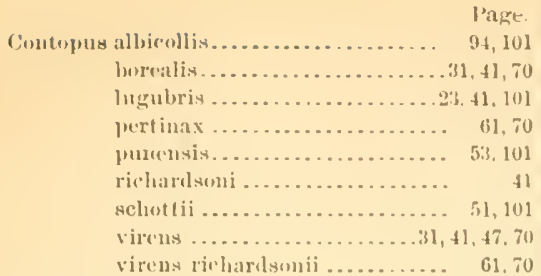

('onurus astee ...................27,52,71

chrysogny. ................ 21

hothmamui ................. 43

holochlorus brevipe............ 55, 58, 64

prtinax .................... 10 .

potzii........................43,69.71

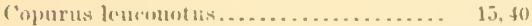

Comethrura alligularis............... 13. 101, 105

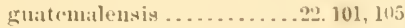

(orvi1 $1_{i 1} \ldots \ldots \ldots \ldots \ldots \ldots \ldots 11,30,39,49,51,61,69$

Convus amcric:tuns ................. $31,49,100$

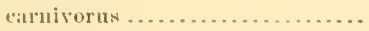

corax carnivorus............. 61,63

mexic:mus ......................

өssif mugus.....................

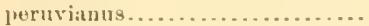

Cory, Cliarles li.................... x

Costa Riea....... X, 22. 24, 27, 34, 35, 36, 46, 55. 58, 66,

67, 101, 102, 103, 104

Cotinga amabilis.................. $1 \times, 41$

Cotingidla. ......... I1, 15, 14, 21, 26, 41,51,57, 62, 70

Coturniculus lenslowi .............. $\quad 30$ mexic:mus............35, 101. 105

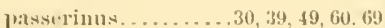

Cotyle flavigastrit ................. $1: 3$ riparia $\ldots \ldots \ldots \ldots \ldots \ldots \ldots \ldots . \ldots \ldots, 37,4 \nVdash, 68$ uropugialis................... 1!, 20, 101

Cotues, I'rot: Elliott. . . . . . . . . . . . . . . 5

Cracida .....................12, 16,21,45,72

Crax globicera ..................... 45,72

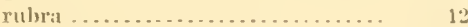

Craxirex univinctus harrisii...........

Creagrus fureatus.................. 7

Crotophaga ani ................. 12,81, $86,91,97$ rugirostris ............ g sulvirostris......21, 43,52,54,62,71

Crypticns platyrhyneluns............ 10

Crypturidla ........................ 45, 72

C'rypturus sallixi.................... 45

Cuba................., 4, 5,7,8,9,17,77,101

Cuculidie.......12, 19, 21, 22, $25,31,43,87,52,54,102$,

71, $79,81,82,81, \pi 6,88,90,97$

Culicivora atricapilla.............. 3 lemberei ................. 5

Cupilonia rupirlo. cupidu palliclicinctus........

Curacas, Is]and of . . . . . . . . . . .

Cyauocitia arecotioulit beecheryj ....

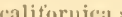

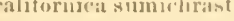
coronatil. ............. crassirustris..................... cristata ................. 301, 49.1100 pulklira ...................... 75.101

Syanocorax lucfnostus.................. lıxแ1) ortoni

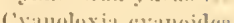

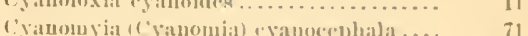
gualomalendis .............. 57, 62 qualulriwolor $\ldots . . . \ldots \ldots \ldots . .6$ vlolireps..................

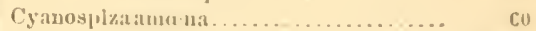
viris .............. 2t, 39, 51, 6t), 69

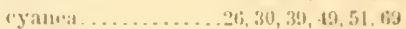

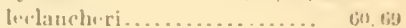
rosita ................ 58, 69, 10: virujulus .............. 60

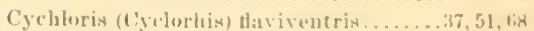
subllivityorns ..... 37

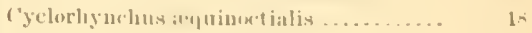
lirevirustrix ........... 15 cim.r.ienp............. 15 subhruming........... 18 sulphureseris.......... I8

Cygnus am»ricatu. ...............

('yubilanius ('ymblihaimus) limstus.... 11, t0

tyminlis cayenu-nsis ................13.41. 72 nueinatus ................. 14,72 wilsoni ................. 9

Cyphorinus bamb]it ................. 14 mutaus .................. 10 fiscialuventris............ If lawrencii ................. . 20, 20,30 lencophliry................. 36 Ioncostictus ................ 14.36, 67 philumela .............. 13

Cyprelitlin. . .31, $4 \pi, 52,71,78,80,45,84, .99,94,9 \pi, 111$. Cypretoides niger ................. sy 91

Cyjeclus collaris.................... 5

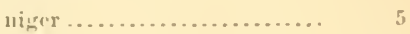

l'yrtonyx massena ................. \$ 64 suluiclirasti .............. 75,102

1).

Darnis earebifulor. 10 nlt rimarina................23, 25, 57,102 venusta.................. 17, $3 \pi, 102$ sp. ! ....................... 13

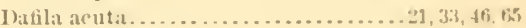
hahamensis ......................, nt, 92 Damophila amahilin................ 10.43 Daption гарепнік................... Demiegretta lentemgantrat leneopyrmna. Iis lenvopyrminat ......... 6. ludwvi ianta.............31, 4,5,5i

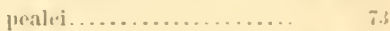
ruf:ı ..................53, thi, $\mathrm{i} 3$

[w-1)drocinela lumigata ........ ..... 14 rlivacea ............. 17. 112 s1. ?................

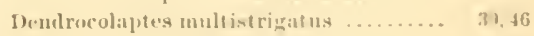
puarticellis . N...tert. 46

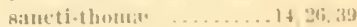

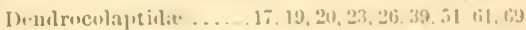
Dendrocrgua autumnalis ........ $2.21,46,65$, it

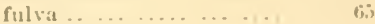
viuluat:i

Dindruca, nev Demalroica.

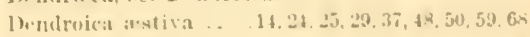
ulbiculliy . 
I'age:.

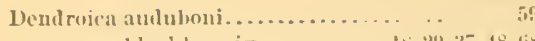
blat.klmurnise .......... 18, 29, 37, 18, is (1)rul.ia ................... 14,50 manulensis ............... $29,4 x$ rapitalis ................. 46. 1102 castimea ................ 14, 29. 18,68 corvuata ..............20, 29,37, the discolor ................. 29. 48 dominiea............... 51,59 dominira albilora .......... 68

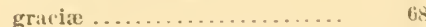

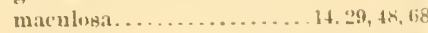
palnarum................ pemsylvanie . ........14, 29, 37, 4, 50 petechia.............76, 78, $42,83,06$ petechia melamoptera ........8, : 91, 102 potechia ruticapilla.......... 91 pinus ................... plumbea ..........75, 76,78, 89,91, 102 rutigula ................. $8 \vec{\imath}, 91$ striat a .................... . 29. 48, 95 timrina . . . . . . . . . . . . . . 29.48 vieilloti .................... $37,51,59$ virens. ....... 10,29,37,68,76,7x,91,95 Dentromaues homoclirous ........... 17 Dendrornis (hurueirostris.......... 51, 64 "rythopygia ............. 39 gutt:it 119............... 10 Jachrymosus ............. 17, 102 lawrencei ................. $x$ mentalis ..................35,61, 102 nawa $\ldots \ldots \ldots \ldots \ldots \ldots \ldots . . \ldots, 20,102$ pardalotus ...............20,26,39 tenuirostris ............. 10

s1. ?...................... 10

Dendrortyx lencophrys ............... $\$ 5$

Viablotin . . . . . . . . . . . . . . . .77, 79, 100

1)iglossa plumhea.................. 37

Diomeda brathyura................. 5

chlororhynehus ............. 6

exulans......................

fuliginosa................. 6

Diplopterus excellena............... 12 nipvins ...................25,43.71

Dolichonyx oryzifolns ............22. 30, 49, 96 Dominica, Jaland of . . . . . . . . . $75,76,77,7,79$, $85,92,93,101,102,103,101105$

Doricha bryant: ....................35, 42, 10:2 enienra....................

Doryfera ludevisia .................. 42

Dromocuceyx mexicanus............. 19 rutigularis ............. $36, b_{1} i_{2}$

Dryocopus mallerbii .............. 12 scapularis ........... 16,43,52, 62, 71

Duck, Black, sp. ?.................. 9s

1)ysithamus rufiventris . ........... 23, 102 semiciner(11s........... (1) striaticops............23,40,102

I..

Ectopistes migratorins................31, 49. 100 Ecuador ............5, 22, 34. 35, 53, 54, 58, 67, 75 . $99,101,102,10: 3,104,105$

Elainea arenarum 40
Elaina phiriguensis ................ Page. (riutzii .............................. macilvainii .................. $55.10 \%$ martinie:a....76, 78, 80, 85, 88, $49,91,93, ! 96$ placens.................. $15,40,51,57,61$ somitlava .................25, 26, 1112 subpagana .................. 15,25,40

Elanojdes fureatus.................. 9,11

Elliot, 1), G........................

Eubernagra chlorira ............... 60

conirostris ............. 16

rutivirgata .........4, 4,51,69, 102.115

st riatien

sujerciliosa............. 39

Limpidias fuscus.................... 31,47

Empirlonax acalictus................ 9,31

atrirustris.............. 55, 102

bairdii $\ldots \ldots \ldots \ldots \ldots \ldots \ldots . .6 \ldots$

braclytitrsus ............ $\quad 15$

dithicilis ................ 57

tlavescens ..............2:3, 41,112

flaviventris ..........21, $31, \$ 1,47,70$

Harivenlris ditlicilis........ 61

fulvipectu............... 55, 102

griseigrularis ............. 18, 102

griseipectus............. 53. 102

hammondi ............. 70

lawrencej................ $x$

minimus ............... 31, 47, 70

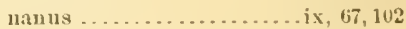

pectoralis ............... $: 45,102$

trailli . . . . . . . . . . 21, 31, 41,51

trailli pusillus............ 61,70

Engyptila fulriventris ............... 95, 165

gannseri ................... 94, 102

viuaceitnlva............. 95, 102

w'llsi .................94,97, 102

Ephialtes cloliba ................... \& 3

Eremophila alpestris chrysoliema ....... 69

cornuta.................. $\quad 80$

Freunetes oweidnutalis ..............23, 102, 105 petrificat 11 s ...........83,84,90,92,93 pusillus . . ................22,32.97 pusillus of cirlentalis ......... 73

Evismatura douninica ................ 65 rubitit...................33,65,98

lirythronotil edwardi ................ 10,4:t

Eusometiscristata .................. 12 spodocephala ............... 38

Enet hia hiculor .................... 90 Eugenes spetabilis.................. 42, 55, 105 Enlampis chlorolamus............... 22 holonericens............... 76,78 , $80,82,84,85,89,89,91,96$ jugularis............. $76,7 R, 80,8 ., 89,91$

Enmomoti вupereiliaris..............11.52.70

Eupherusa chionura .................. 43 cupreiceps .................35, 43.102 egregia ................... 46 eximia.................. 43,46 nigriventris ...............36, 43, 102 nivejrauta .............24, 35, 102, 105

Fuphonia aftinis ................. $38,51,60,63$

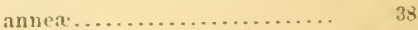
crassirostris.............. 
Suphonia elogantiasimat

$1: a g r \cdot$

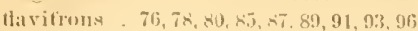
grilitho. grarilis.................. 38 hirmulinacea .............. 12.38. 68 humilis ................ 38 lateicipilla. minutil .......... purpurea. rutivertex.

35,102

ritiserext.

Eupsilostoma jusillum.

Furvesga (Euripysa) lerlias

miljor........ 19,46

Forypgina (Euripygirla*)

Euro] $x^{2}$

Euscirtlumus squanieristatus.........

Euspizat auneriume - a Euthlypic

Eutoxeres apuil salvini

43,40 40 16

I.

Falo anatım.................... $\quad \Omega 8$

volumbarius ................ 97

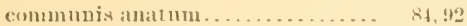

perengimu anat um . . . . . . . . . . .

peregrinus nigrienps . . . . . . . . . $5 t^{0}$

sp ! . . .

Falconitlit, $1,13,17,20,25,27,29,44,46,47,52,54,56$. 53. $63,72,79,81,82,84,86,8 \div 4,9: 1,57$

Florida ................... 1, 2, 6. $7,8.103,104$

Florida ejerulea, $16,31,46,53,65,73,81,83,84,86$

$\pi x^{2}, 92$

Florisuma melliveria .............. 10,42,5i, 62

Fly C'ateher, Lawrence's............. IX

Forest and streinu ............... 76.92

Formicarida $11,14,13,19,20,23,26,35,40,51,53,94$

Formiı arius analis................. 15,40

hoftmanui ............ 40

pallitlus ................ 94. 102

Formuirivora buceardii ............... 18,40 grisidgula .............. 94, 102 melarua.

quix"msis . . n

selisticolor ............24, 40, 102 virgata ................ 19, 20, 102

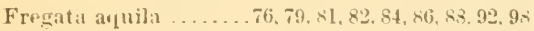

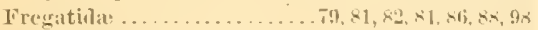

Fregettal lawrencii................... IX, 6

Frinuillida, 11, 16, 18, 21, 26, 30, 38, 19, 51, 57, 54, 69,

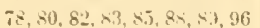

Fulica anurieana ............23, 33, $46,50,65,74,93$

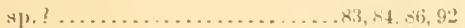

Fulix attinis ................... 33, 4t. 53,65 (n)llaris .................... 33,65 marila .................... 33,65

Fur comntricy................... 6. 7.8

\section{G.}

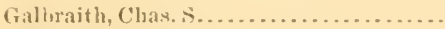

(iallula melamogenia.

(ialbulistar

41

Galeoscoptes ramolinemsis............2s.49. 51

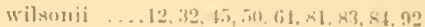

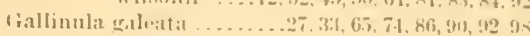
mitrtinita................ $13, \ldots$

frambetta tha

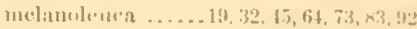

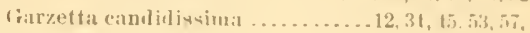

(6.5), $73, \times 1, \times 3$ 8fi, 13

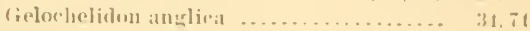

(icorocery mexicimus................52, 6:31

viatticluk..................

tieorgia............................

licothlypis manegilliv ray i .............. $\quad 37$

philamlelplial ................14,29,37

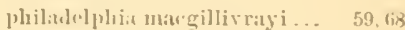

polimwplata .............. 51.50

triclıas ...........29, 37, $13,51,59$

fieotrrgun albiventer. ..............27, 14, 102

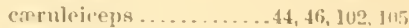
chirigumbis ............... $40,10.5$ costaricunsis.............. 4t, 10:

lawroncii ................ montana ......44, 76, 79,81, 86, no, 92,97 mystawea .............. 99,92 rufirentris ............... 66, 102

veragurusis................ 35, 111:

violaceal................ 1!)

Goranumiza werulese+ns, ........... gracilis niger .............

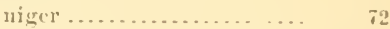

Girand, Jateol, I'.., jr................ IX, I

(ilaucidium lervuginem (ferruginium).. 63,71 gnuma. .............. 41.71 infuัเatum ................

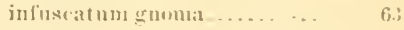
siju

9

Glatu is aneus ...................... 36, 42,102 allinis..................... 5,102 hirsutus ..................., 85, 91, 36 ruck.ri ................... 14,42

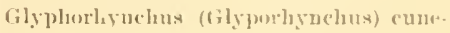

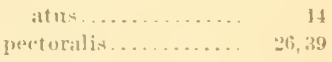

Golman, F, In Cimt.

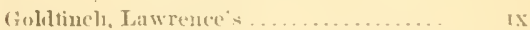

Gouldiar wouversi. . . ................ 14. 12

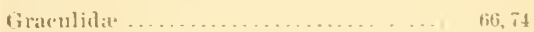

Graculns varbo .................. riucinnatus ............... 7 dilophus ................. 73 ?

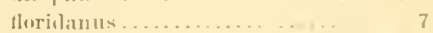
mexicantu............

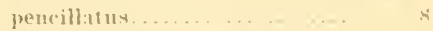
perspivillatus f... violiwents.

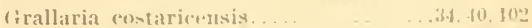
dives

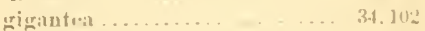
jwipicillatil ... ... 13, 15, 41), 102

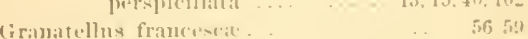

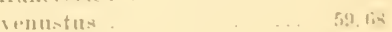

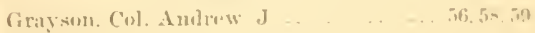
lirm-nl:mul ... 6. 7

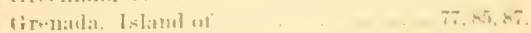

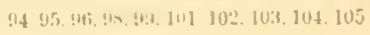


Gronalimes. The...

Gimisl:u

Ginateloupe, lslanilul.

$89,90,95,101,100^{\circ}, 104$

Gilatrmalit ................22, 36, 17, 101, 102

(iniana.......................... 93, 101, 103

Guirica eisrulea (eilrula) ....... $30,39,51,60,69$ roncreta ...................

parcllinit................... 60, 69

(Gull, White-winged.

(imullich, H)r. John

Gymnocichla muliceps

(iyunoglaux lawreneii

$$
\text { nowtoni }
$$

nndipes

Fyjuarelus papa

\section{II.}

Hatrostomus aglaie ................41,51,70 iglaia athinis........... 57,62 alliventris ..............35,62.102

II:rmatopordidia (II:ematopirlax) .32,45,53,57,61.73 llienatopus palliatus .........32, 45, 53,57,64,73 lawrentii ................ melanotis .............. 47,105 rufiscens ............... rufieauda................. 69 sumichrasti . ...........54, 69, 102

IIaiti.

Haliatus (Italiä̈tus) leveocephalus. Haliplama diseolor ................... 23.

fuliginosa ............... 27

fuliginosa erissillis ..........57,5×, 66

Harelıla glacialis..................... 33

Harpagus bidentatus ................

ficiatus................ $47,10^{2}$

Harpia destruclor .................. 46

Harpiprion earemensis............... 19

Harporbyu.lus eurvirostris . . . . . . . ... 59, 59

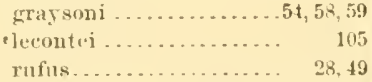

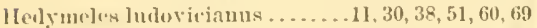
mel:mocephalus........... 60

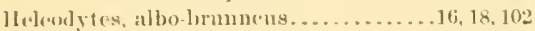

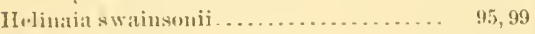

1h.dioloxa lenryi ....................3., 42,162 jarula ................... 12

Jlelimmaster eonstanti ............... 42,71

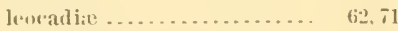

longirustris .............. $\$ 2$

pisllidiceps............... 43,71

selateri ................ 20,13

spectalilis.............. 35, ]02, 105 striatic .................., 10, 102

ILliopielica rastancucaula........... \&. 102

H(diornis fulica .................... 13

Heliot hrix anritus ................... 10 barroti

10.42

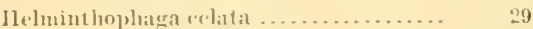
(heryopterat ........10.29.37,4s liawencii peregrini ......14, 24, $29,37,68$ pinus............. 29.48 ruticapill:ı ......... 29.6n 95.99 lawrencei ........... IX

$11 \cdot$ imitherus veruivorus.............29, 37.50

Hemiproene minur................. 91, 102

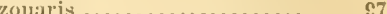

Hemitlyylaen (Homytlyylat'is) hoftmanni.. 43

Ilt-nicorichla auresapilla .............. 25

IIerolias enrelta ...........21, 31,45,57,65,84,92

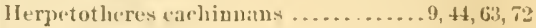

ILerriık, IIarolel.................. Ix

Jlesperipluma vespertina ............. 30

Heteropelua verplacis (roropacis) ...... 18,41

IIitks, Frut ...................... 24

II irrofaleo islamelicus................. 28

1fimanlopms nigricollis . 21, 32, 64, 73, $81,83,84,92$ II irumdinidie.... 13, 19. 211, 29. 35, 37, 48, 51, 60. 68, $7 x$, $80,85,87, \times 9.96$

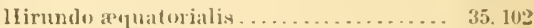
liveolor. . . . . . . . . . . erythrogastra .............. $\quad 96$ horr.orum.......13, 22, $29,45,60,68,89,91$

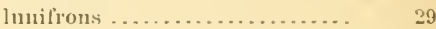

Ilistrimicus torpututus ..............

Honduris. . . . . . . . . . . . . . . . . . . . Pritish .................... 36,55,101

ILumming birl, Lawrence's .......... x

II yorochelinon tissipes................34, 66. 7t plumbea.............. 7,13

Hylophilus acutieaudus ............. 27, 102

aurautiffrous ............ 14. 102 cinereiecp................ 37 decurtatus................ 37 , 68 ochraceiceps .............. $3 i$ pusillus ................14. 25, 37, 102 viriditlavus ............... 14,102

Hy]otomus pileatus................. 31

Ify hantes balt imore ...............

Hy jocnemis naroides................ 15, 10

schistacea ................ 15 sp. ?................... 15

Hypotriorelis albigularis ............. 72 aurautius ............. 52.56 columbarius .......28,44, 47.56.633

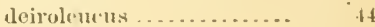
fumoralis............ 63., 72 rufigularis . . . . . . . . . 17.56, $\left(i_{3}\right.$

llypuruttila lutloni ..................

\section{I.}

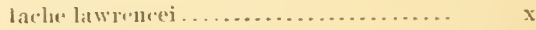

Ibis athat . . . . . . . . . . . . . . . 32, 45, 6if, is cuaramia.................... orlii ......................... 32

Ibis, The............... 16. 19,67,74, 75, 77,93

Iby.ter amerivinus .................. 9,44

Isteria longirandis ................... $3,102,105$ virens...................... 37.51 .68 virens lom ricanda . . . . . . . . . . . 60, 105 virittis .................... 29,48

Irteridar ....................... 15. 23 , $26,30,39,49,57,61,69,80,85,88,89,96$

Icterus auratus ................... 54 baltimene: ................. 15, 24, 30, 49 lonanil . . . . . . . . on. 91 cucullatus .................2, 4,9.6] 
Ieterus dominicensis

Page.

formosus. . . . . . . . . . . . . . 55, 69,102

girandii . . .

11

graysomi ......................

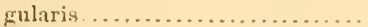

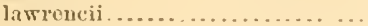

melauocephalus.............. 69

mesonuelas...................11, 26, 69

oberi..................... 93, 102

pecturalis ................. $\quad 89,69$

prosthemelas................. 69

pustulatus................... 61

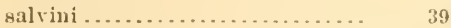

spurius .................. 15, 21, 30,49

spurins aftinis ..............61.69, 105

wagleri

Ietinia plumbea.

61

Iomolatima firontalis

9,7,

Ionoruis martinica.

5,102

98

\section{.J .}

Jacamerops (Jacaneraps) graudis.......

Juliamyia (Juliamya) typica.

10

Junco hyemalis.

10,43

\section{Is.}

Klais guimeti

I.

Labrador

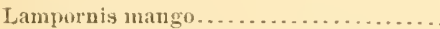
prevestii . .

25,42

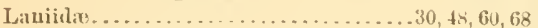

Lanjo aurautius.

lawreneji...

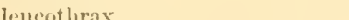

Lauirireo tlavifroms. solitarins...................

68

$x$

Latridif...6, $27,34,50,53,57,58,66,74,79,82, \times 3$. $84,86,88,90,98$

Larus argentatus

6,9

at ricilla..............83,84, 87,90,92, 94

ralifornicots. . . . . . . . . . 4, 6, 74. 102, 105

chaleopterus ...................

dlelawarrusis ...............6, 34, 66, 74

glaucescens ....................

ylatıеu. .................... 6,34

lutehinsii.................... 34

lencoptrins .................. $\quad(i, 94$

marimus ................... 1i. 3.1.50

occislentalis .........................

smithsmianus ............... 34

suckleyi..................... 5.6. 102

Lawrence, Geo. N., Biograplical skutch of vu

Lawreuvia ...................... I. $10^{2}$

Legatus albienllis.................. 18, 2.5.40 variøgatus ................. 15,10

Lepidemats speciosa ................ 12

Leptopogon amaurocephalus..........

tlavorirens............. 18, 10.2

Leptontiha albifroms . . . . . . . . . . . 52. 5ti, 63, 72

lomapatrti.............. 55.10 .2 cassinii .................36, 44, 102 fulvivintris . . . . . . . . . 94, $10_{2}^{2}, 105$ l'age.

Leptoptilit riottrif.... ............ +t. 102

vireauxi ............... $\quad 16,4$

Lesthia amaryllis..................

ortoni .................... 54.103

Lenerpezza bishopi .............. $77,80,91,102$

Inucopternis priusp p..............

s:-miplumbens .......9,44,46, 102

Limusa ferloa ................32,53,64, 73,97 hudsonica.............. 32

Linnaran Society of Jew York........ viii, 99

Lipaugus allogrismus .............. 21, 102 holerythrus ............... 15,41 rufe.*iens................ 15,41 nuirulus ...............

Lohipes liyperborms ............... 73

Long Island, N. Y ......... 1, 2,3,4.7,28;1,75

Longi munes....................

Lopluodytes ancullatus ............. ;3\} biculor .................

Lophornis delattri ................ 17

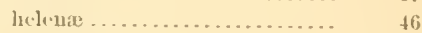
gambellii ................. if

Lophostrix stricklindi............... it

Lonisiana ........................ . $7,7,95,99$

Loxia americana ................... $\quad 30$

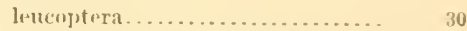

Loxigilla noct is......76, 7s, $80,82,83,85,82,89.91,96$ porturicensis grandis.......... 93. 103

L yetum of Natural II istory of New Fork.

Anuals of . . 1. 2, 3.4, 5, 9, 13, 17, 20, 22. 23, 24, 25, 2R. $34,35,36,50,53,54,55,5 \%, 66,67 \pi, 75$

\section{II.}

McCown, ('apt. J. 1'

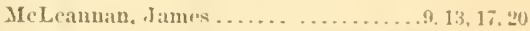

Maerorhamplus ( $\mathrm{Mlacror}$ amplute griseus 9,18 ,

22. 32 grisinus scolopiceus..... 73 scolopitcens . . 1, 4, 9, 32, 45, 64, 103

Malan'optilit rostari(ensis . ........... frontalis................. 13

inornatit. ................. 20,12

pimamensis ............... 10

r.ropacis (verapatis) ....... 2fi, 42

Mareral americallil .................. 33,05

prolope...................... 33

Margarops alloiventris ................. \$8. 103

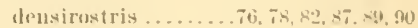
dominicensis . . . . . . . . . 92. 93, 1113 fustatus ...............

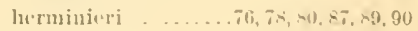

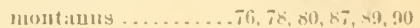

Markarornis brunnescens.... ....... gutt:1ta............... $2: 103$ rubiginosa ............23, 3!!, 103

Martiuingu. Islaud of ................., so 103

Marylaud ........................ \&

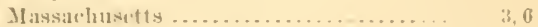

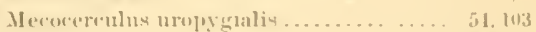

Megarliveluts mexi anuy ..........26, 41.51, 70

Melamerjes erythroephalus ..........31, 47, 1110 tirmicivorus ..............

l'hrminieri............ 84,91

Melanetta velvetina ................

Mrelunt is cirrulescens . . . . . . . . . . . 56,59,67 
Mremagriuia"

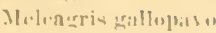

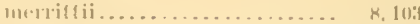

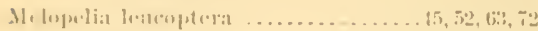

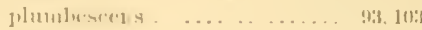

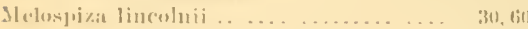
meludia ................ \$30, (!)

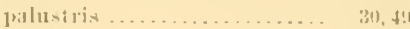

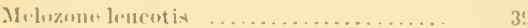

Mlergulus allo ... . . . . . . . . . . . .

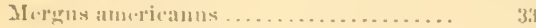
s.r.

Mrrulit migratoria..................

Mlefillura quitensis . ................ 5t

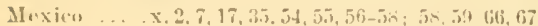
$-74,75,94,96,101,102$ 103, 104. 105 (iult of ....................... $6,7,103$

Miørastur gilvirollis................. 1: poliengaster .................. 13

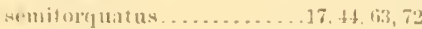

Micrathene whitneyi ............... $58,(i 3$

Mierochera allmeoronata ............ 42, 10. farvipustria . . . . . . . . . $42,10$.

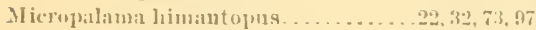

Midhllo states ot Cuited states ........ i

Milvulux furficatus................. \& \&1, 71

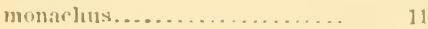
tyrannus...................25, 11.96

Mimns gilvus.............. gilvus lawrenerei ............. $x$ gracilis........................ 50,67 Iuelanoptr.rus................ 2. 103 nigriloris.................. 55,103 jolyglottus................2x, 49, 56,5 ) polyglottus cilulatum ..........

Mionefles ismimilis ................ 15,40 (bleaginus ............... 40 olivaruus ................ $\$ 0,203$ striativollis .............. 15

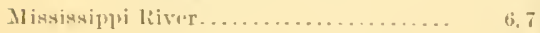
Valley................. 1.103

Mlisмm liver..................... 6,8

Mitrephorns aturationent ris.........24, 11.10 .3 phitocereus ............. $\$ 1,61$

Muintilta varia . .......... 14. 24. $29,37,48,59.68,95$

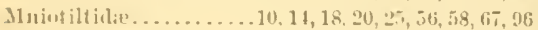

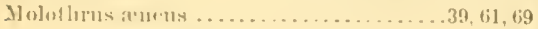
perori.s.................. 30,49

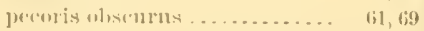

Mlomotilla . . . . . . . . . . . . 10, 15, $55,41,52,62,70$

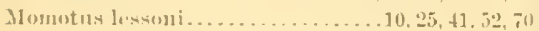
martii .................... 10,41 11, xi subrufesrens $\ldots . . . . \ldots \ldots . \quad 13$

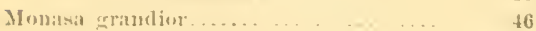

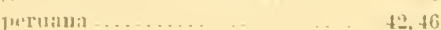

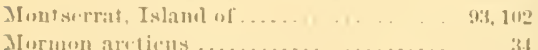

Jirmon ardicus...

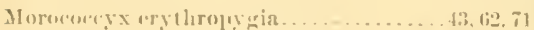

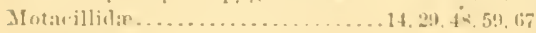

Mlinric spla roronatia. lawernevii

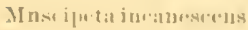

Musrivora maxicitnat............ .11, 15, 41, 51, 70

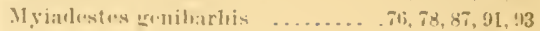
unclimen. . . . .

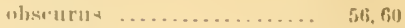
silvilims $\ldots \ldots \ldots \ldots \ldots .77,80,91,103$

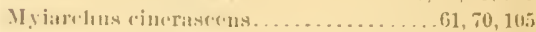

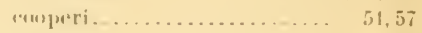
rrinifus ............ 15, 31, 41, 17,70 crinisus «nер.ті ........... Hatumulatu............... . .6. 70, 103 1:1 wr.ur.ii ...... $1 x, 36,41,51,57,61,70$

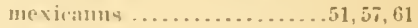
niøri,apillus ............ 41 nigricu|ls............... 15 olw.ri . .75, 76, 78, 811, 85, $85,91,95,96,10: 3$ pinamensiя ..........9, 11, 27, 41, 103

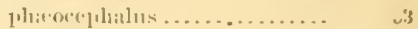
sel.1t(1.1.................. remenclensis ............. 27, 103 yne:ltancusis .............. 5.5. 113 Myiobius atricaulus................19,21, 103 1) arbatu世................... 15 apitalis .................. 41

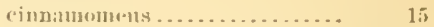
"ry1] rurus .................. 18,41 s!1] ] ]

Myimliontus carruleserns.............. 68 ('in)ulensis............... 18, 49.37 mit mif us..............20,29,48,51 pusillus ................. 29, 37,39 pusillns pilmolatus......... 68

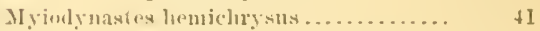
Jutriventris ............... 40,61.70 mol,ilis ..................11, 25, 40 sujerciliaris ..........35, $\$ 1,103$

Myiozutetes caycumensis.............. 11 columbitmus ............. 15, 25

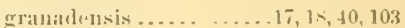
gramlis................... 55, 103 inuruatus................. 54, 193 marginatus............ 19. 21, 40, 10:3 rutipwnuis............... 54. 1133

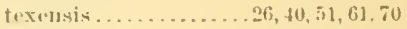

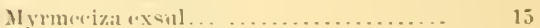

ferrugiura.............. 18. 103

immatemlata $\ldots \ldots \ldots \ldots \ldots \ldots$. 40

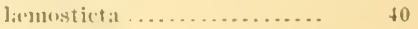

longipres.................. 15

stivtolıtrit ...............23,40, 103

Myrmelasfex corvium ................ 19.20, 103

Myrotherula (Mrrmetlerula) al higula . 23, 40, 103 lulvis'utrik............ 18, 40,103 melarnil............. (1) nodlestit .............. 40. 103 pymeal ..................... 14 surinamensis............ 11 s1, l $\ldots \ldots \ldots \ldots \ldots \ldots \ldots \ldots$

\section{N.}

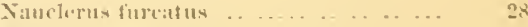

Xertijs fuligintsus . . . . . . .

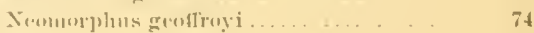

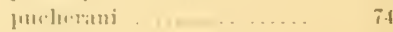

ruti unis.......... it

milvini 
Nettion ramolinemsis

1'an. creeca

$33,4,7$

Now Engramd sitate.

Now Gren:alia ....4. 8. 9-13; 13, 13-16, 17-19; 19 $20,22,21,27,35,36, .46,54,55,5 \mathrm{k}$ $6,6,93,91,95,101,102,103,104,105$

New Jirsex ............ Ix 1, 2, 3,6, 7, 28-34, 101 Now $\$$ Iexien ........... ........... 8, 102

Now Providenes, I slam of . . . . . . . . . . 75,104 New Tork . ........ 1, 2, 3, 4, 6, 7, 22-34, ti $50,75,94$

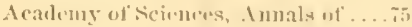
$77,8,3,93,91,95,95$

City, Central l'ark......... . 1750

rity, Vicinityot ............. 3, 2x-31

Island, $\mathrm{X} . \mathrm{Y} \ldots \ldots . \ldots 28-34,17-50,9 !$

Sowf

lianks of ................

Nica ranua .....................21, 25, 101, 104

North America, Allantic coast. of .......

Northwest coist of .... (i. 7

Paeritic coust of .......5,6,7,8, 23

Western coarst of ....... 8

North Carolina.................... 7.5 .5

Northern States of the ["uited States...

Nothererris sallivi.

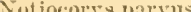

Nowascotia.

(i1, i-

27

Numcnius borealis........................ ludsonicus .23.32, 64, 73, 83, 81.86, 92, 94 Jongirostris . . . . . . . . . 32, 61, 73, 81, 12

Tumila meleagris

it. 92

Xinmiclulas

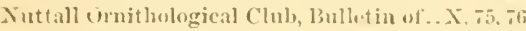

Nyrtale acaticit................... 28

Nretua nivea .................... 2x,

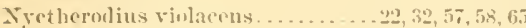

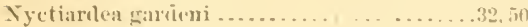
grisea nieria............. n:pia................. violacea................

Nyetibins graulis

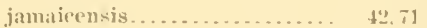

Nreticorax riulacens................

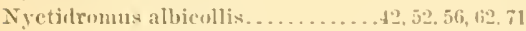
americinns ............. g

couranensis .

\section{O.}

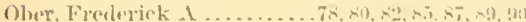
(kennitus oreanicus................. Orthoïcas (Oehthoeca) flavivutris....... 4s. 10:3 rufomarginatus ... 5. 5. po:

Orralus wagleri.

4. 10.

()louterplums quiamensis . . . . . . . . . . .

guttarus ................
leucolimmus............

lencolirmus.

melanotis. .

veragnemsis

()iilicntmus listriatus.

OLio liver.

Oillemia amıric:ma.

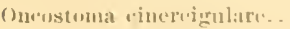
wlivitwial.

Oporornis atrilis. formosis. .............., 2y, 37, ti,
(Mrehilns at rimapillus ....... .... tiד. 10:3

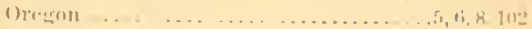

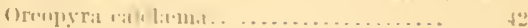

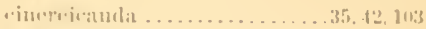

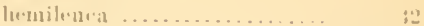

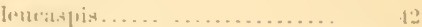
vemusl:1 . . . . . . . . . . . 35. 12. ]113

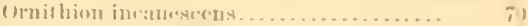

(1)ritlida matcilli ................... 52

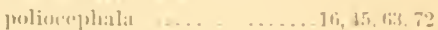
rutivauta ............... vetulat .................. 24. 42 warleri ....

()rthorlyyu'hus crintatus..........55 $\times 7,91,31 ;$ 1.migraus............ 7... \{103 "xilis ....2.2. $76,79,82,44, n x, 4991$ ornattus ............... sil), 91

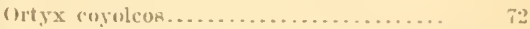

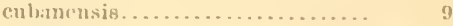
graysoni .....................35, 64, 103. 105

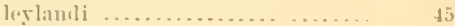
nigrogularis .................. 52 t('xauus ..................... 3, J113, 105 virminianus ..............31, 50, $8:, 7,92$

Oryzoborns a thiops................ 10.

Ostinops r ristatus .................. 11

montezumi................ 11.39

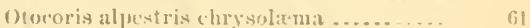

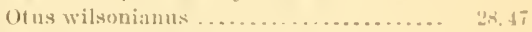

Oxyechus vomiferus................

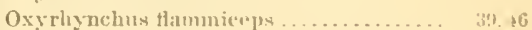
trater $\ldots \ldots \ldots \ldots \ldots \ldots \ldots$ to

l'.

1'arhylh:amphus eimuruiventris ....... ciucrens............ 15

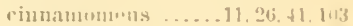

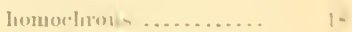
marerimus ... ........ purvelurupteras. sp. ? ............ 11

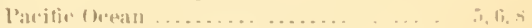

laiilmoul Explerations

l'agophila br:aclivt.1ri .............. ; rlmmea................. ;

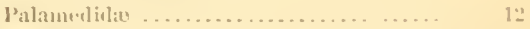

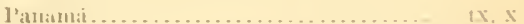
13, 9. 13, 16, 19, 21, 27, 55, 101, 102., 103, 1613, 105

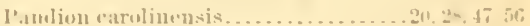

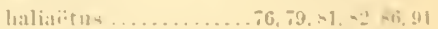

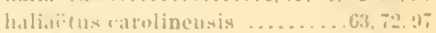

l'antr.rpe insignis. . . . . . . . . . . .

l'anyehlora parvirontris . .... . . . . $: 7,103,1115$

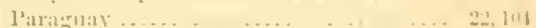

l'arillir ....

l'arra gumostmua .......

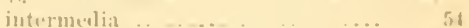

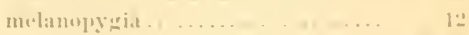

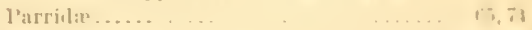

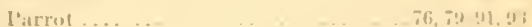

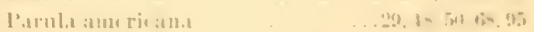

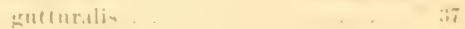
inem 11:11.6

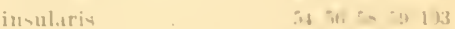
pitiaymui.... ... 53 
Pneri,

P'arus at rir:apillus. carolinensis .

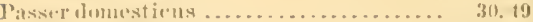

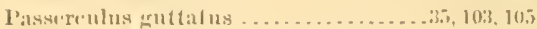
s:iviluna. ............... satramua alaulinus........

J'asserella iliat'a .................... 31, 4!

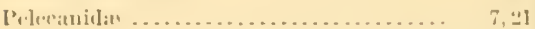
$33,53,66,71,79, \times 1,82, \times !, 86, \times 8,98$

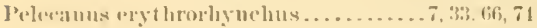
fusc.us .............. 66, 74, 70, 79, +1, 82, $81, \times 6, \times 5,92,9)$

Politlnit ameriona ..................

Polionetta wrspiq.illat: . . . . . . . . . . . .

P'mblulinus prostlsemelas

I'(')

$21,45,63,72$

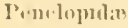

$15,52,6: 3$

P'musclyania

$3,6,8$

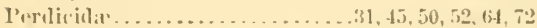

J'risurrus eanatensis................

Prrissuglossal ligrinil . . . . . . . . . . . . . .

l'ristera cinerea.................... 1:, 4

munkletura ................ 45

I'eru. ...........35, 54, 55, 74, 75, 101, 1112. 303, 104

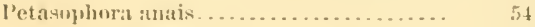

ryant is ................ 42

thalissina............. $5 \vec{\pi}, \mathrm{fi}$

Petrel, Black aud whito Stormy ....... ix

Pretrochelidun alhine: .............. 20, 108, ]115 leturopterit ............ 13 lunifrons.............. 13,60 swainsoni

Pencara astivalis botleri. mexienatia rufieres bourardi.

Peroumetery capitalis

J'hires brua cuviuri.

I'hä̈thon itt therens .........23, $60,81,86,90,92,98$ flavirostris........ $8,23,76,79,84,8, .92$ tlave-anrantil1s ................ 8,103

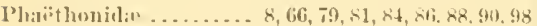
Pliathornis adolphi ................ at rimentalis............ 5. 50.3 rassinii................. 35, 103

1'mil:0 ...................

mirynomu. ...............

longirostris...............

moorei.

14,42

nigrivinetus .................

villesus

5,103

5,103

5,1013

Phatacroreraridlat. .

7,33

$: 32,73$

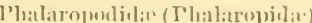

Phataropus inlicarius.

$$
\begin{aligned}
& \text { hyperborells } \\
& \text { wilsonii...... }
\end{aligned}
$$

Pharomacrus luts-rinuo......

Pluevetious ehrysopmolus.

$$
\text { tibialis ........ }
$$

Pheugopulus at rogularis. fastiativemluis

Plhibhela minor...............

Phikmarhus jugnax

Ploilurtyx fasciatus

pliblyor rufolnrumuens. virgatus
60

115

2

\section{I}

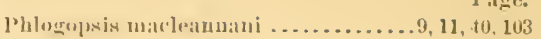
l'hu'uicut hraupis (l'huenivothraupis) car-

mioli .............. 38,1113 erist:ttit............ $61 ; ;, 103$

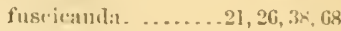
rubievidles.......... 11, 68 vilute: $\ldots \ldots \ldots \ldots . .36,38,10.3$

Plonipara lieoler.....................76, 78, sil xi3, 8:1, 85, 87, $82,891.91$

l'nuosal ................. 58,103 gutturalis ................. 1: pusilka .................12, 39,51, (49)

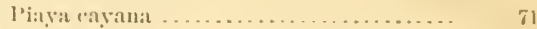

mehleri .................. 43,51

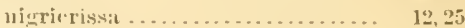

rillihumlus ................. 62,71

rutila ...................... 12

I'irid: $1^{1}, \ldots 12,16,16,20,21.27,31,43,47,52,54,5 f, 62, \times 9$

Pirolaptrs allinis.................. 39

lrumucicapillus...........

compressuis ................ 39

lintatiops................. 39

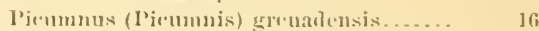

l:ıwrnii....... $x$

I'ic'ns lworealis ...................... 31

rhrsixanlosus................ 5

h:ırvisii..................... 43

,iarlinii..................... 4 ,

pullestrns ..................... 31,47

sularis ..................... 52,56, 11

sealaris grazsoni .............. 62

villosils.................... 31

Pigron .......................... 93,94

Pilluerwius pileatus................. 12

Pinieola cinatensis .................. 30

Pionius coccinicollaris............... 18, 103 larmatutis.................. 12,4

1urustrums......................... 12

senilis.................... 27,4

albicollis .....................

(.rythroplithatmus. . . . . . . . . 30

reythrophthalums cammini......

fuscus crissalis............... 60

maculat 1 . ................... 69

Pipra cinnumomea ................ 47, 1103

lencorrhon................... 41

mentalis................... \$1

Piprilia...................... it

I'iprites gristiceps................ 41

P’tangus alhevittatus.............. 17, 18, 103 flerliamus ..............40,51,61,70 gablii ................... 67, 103 livtor....................... 15

Pithỵs birolor .....................20,40,10:3

l.tle aspis ..................... 15

rufigularis .................... 11

l'ittasomat michleri $\ldots \ldots \ldots \ldots \ldots \ldots \ldots .11,15$

Pitylus (I'ytilus) groxsus ............ 12. 3\%

humeralis ............ 35, 104

guliugaster ......... 38

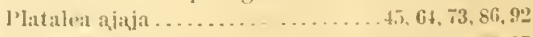

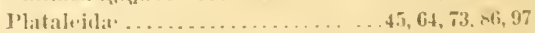

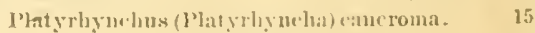

rancrominus........... 40 


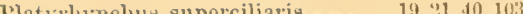

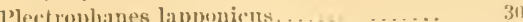
m(ฯ)พnii... ... ...3,4, 103,105 nivalis.... . . .... $30,4 !$

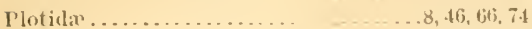

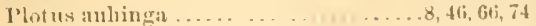

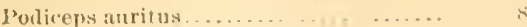

auritus calitomicus - .......

eilitiornicis .............. \& \&

clarkii..................., 103, 105

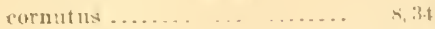

rristatits ................ $8, \Omega 4$

dominirms ..............

griseigria ................ $\quad x, 34$

hullucllii ..........

nceridentalis ..... ........ 108, 105

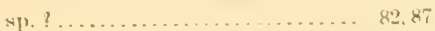

Porlivipidla $\ldots \ldots \ldots \ldots \ldots \ldots 46,615,74,52,44,87,98$

Podilymbur deminicus.............. pulivep. ...........8, 34, 66, $84,92,9 x$

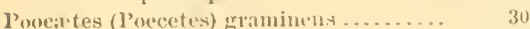

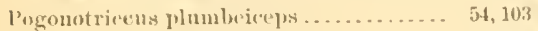
zelerloui .............. 46,103

lobliontila albiloris..................

alliventria $\ldots \ldots \ldots \ldots \ldots \ldots . .65,103$ (ax rule: $\ldots \ldots \ldots \ldots \ldots \ldots .28,50,59,73$ mel:mura ................. 4. 103

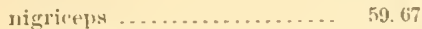
plumbic(e) $\ldots \ldots \ldots \ldots \ldots \ldots .27,103$ superciliaris .........13,14, 25, 36, 103 Polyborus andubui . . . . . . . . . . . . . 44, 52, 56 thame anduloni ............ 63,72

Pophyrio matinic'1s (utartinica) ........65, 81,92

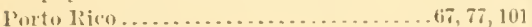
Porzana albigularis ................... 16,105 carolina ................... 19. 33,65, 98 viuorejuep................. 66, 103 conculor .................... $7 t$ guatemalensis ................. 74,105 noveloracensis .............. 33 sp. ? . . . . . . . .

Prionirlyonchus platyrliynchus ......... 41

Procellaria brevirostris . .............. 103, 145 сареимін ..................

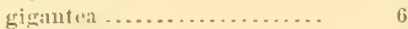
glacjillis ................... 6 lelleorrlion ................. 34 merislionalis ..........., 3, 6, 104, 105

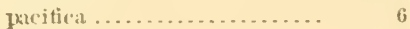

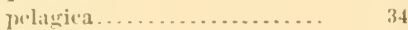
tennirustris .............. 6

Procellaridin .......................5. 34, 79,98

Procnian tersil....................... 99

Progne chalybea ................... 13

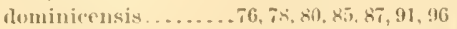
lencoganter ..................... 37,6 . purpurea.................. 29,48 subis........................ 60

Protonotaria vitrut .................. 37.50

Psenderolaptes lawreneii ............. $\mathrm{x}$

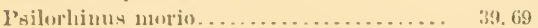
I'sit tacilit . . . . . . . . . $56,58,(52,71.79 .81$

P'sitticulil eyanopratra................ 5t; ti3 Psittacus pulrerulent us

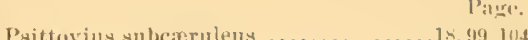

$1:$

Premeyanea rapuleata................

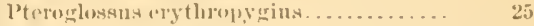
trantrii............ . torq̨uatus ............... 13, 71

Ptologonys (l’tiliogonis) atudatus...... is vincreus ................ 60

P'utlinus anglorum . . . . . . . . . . . . . . . 34

auluhumi................. 9!

cinurens ....................

fuljginusuк................

mitjor .......................... 6,31

obseurus...................

s]p. ?........................ is

Puget Sumul... ............... , 6.8. 101, 102. 104

Pulsatrix torquata ..................

lygmormis alolpli.................. 42

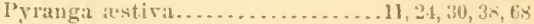

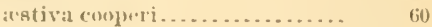

billent:ata...................38,57,60

ergthromelat.11:............ 38

hepation.................... 6il, 68

lulovicitna................. tis

roseimularis................... 91

rubrat.................. 15,30,36.48

Pyrgisomi liarematmu .............. . 39

eabanisi $\ldots \ldots \ldots \ldots \ldots \ldots \ldots . .40 .46$

keintri $\ldots \ldots \ldots \ldots \ldots \ldots \ldots \ldots . \quad 39,46$

rubrisatum..............

rantusii ................ 35.101

lyroceplalus soromat us.............. 4

nехіныны............. 51

nimus ................ 53

rubineus................

mulinens mexi:anus...... 61,70

Pyrrhomitrisuneullatus..............

Pyrrheplatna vimumome

levillei................ i]

graysmit...............35.57, 62. 104 Jietlieri ................. $27,43,71$

l’yrrhuloxia siumata ................ 60

Q.

Querquedula 'ranoptori ............ 6.5 diserers . . . . . . . . .

Querula cruentat ................... 11,4]

Quisealus fortirostriv .............. 46, 104 gualelum

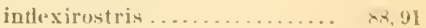
Inmiuosus..........77, 85, 87, 91, 96, 104 nacrourus . . . . . . . . . .2, 4, 2t;, 39,69 major.................... 61 major macroarus .......... 61 uajor palustris............. 61 mexic:uи ................ ритритен............... $\quad 49$ versicolor ................ 30 sp. ?.................... 80

12.

Ii:t!islit 13, $19.22,25$, $27,33,46,50,54,65,74,-1, \times 3,44,81,90,08$ Rallus crepitins .................... $33,90,92$ 
Risllus eleogans.

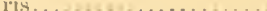

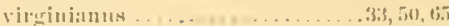

s]).

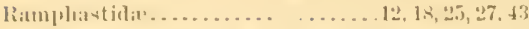

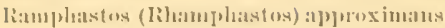

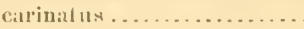

piscivorus ................

towird ....................

liamplucienus ruliventris . . . . . . . . 20. 20 semitorezuatus. .......... 18, 40,104

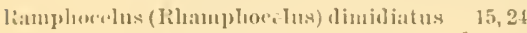
ictoronotus.............. 11

lucialitil............... 15

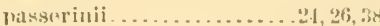

saucruimulentus.......... 38.

Ramphocinclus bracliymus...........

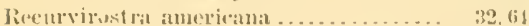

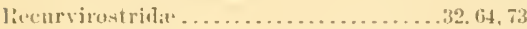

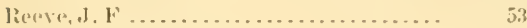

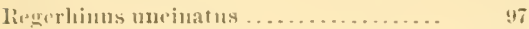

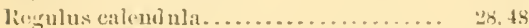

sittritpit ..................... $2 x, 48$

satrijua azteris............... 98 104

lihamploutcron (liamplowmieront) olivit.

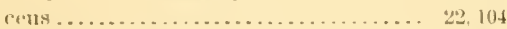

lihodimeichla rosetz . . . . . . . . . . . . 10, 36, 59

Iiluklostrthiit roseit .................. i

Rhyouphilus sulitarius..... $16,32,45,71,64,73, \times 3$

$84,90,92$

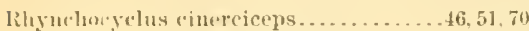

flavo-olivareus......... 21, 104

griseimentalis ......... 40,104

marginatns ............ 47.104

sujphurescens......... 41,46

l:hyn.lophanesmecowsii............ 105

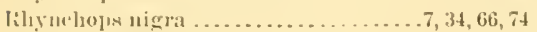

lilinogryphus aura ................ 72

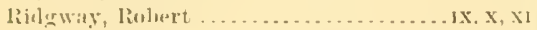

Rissil hevirostris..................

nivea ..................

septentrimalis................ 5.6,104

Irialactyla (tridindylus) .......... 6, 34

lowtrlinmm (liosluramms) sociabilis.... th th

Jiowley, (x. 1) . . .

lowley's oruthologienl Miserllany .....

liujurnis mignirmstris grisucanta.....

linssitm Americit .................. 6,7

Sit. Aulrows Islieml

Sit. Christopher, Islimel of.

St. Iroix, Inlimel ut

Si. Latwrouce, finlf of

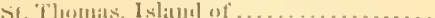

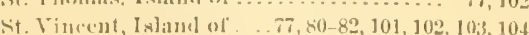
Silluin.tes olsoletus ................

Satlatur atrieceps....................

fulviventris ................. 20.2101

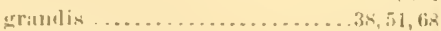

Enadelompensis .......... $70,78,87,89,91$

intermudius .................... 24. 104

milgnoidlo.s................ 26,38

misguแ⿰ .

11
I"age.

Siltitur plumbiredes .................35, 60, 101 striatiputus............... 15,:11

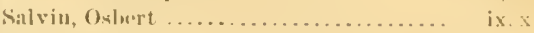

simulpif. . . . . .

Sinto Jouningo ............... ix, 65, 67, 102. 103

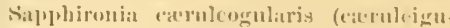

linis) $\ldots \ldots \ldots \ldots \ldots \ldots \ldots$ 14. $1: \%$

lmminosa............... 17.10!

Sarroramplus pat $\ldots \ldots \ldots \ldots \ldots \ldots \ldots .20,16.1$

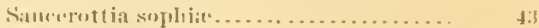

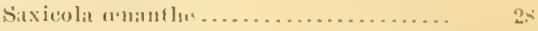

Siximolibte ........................ 28, 48.5?

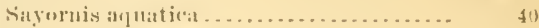

niщricans ..................... 6170

sayus.........................

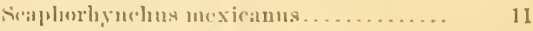

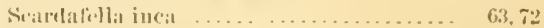

sirlater, 1 . 1................ ix, $x$

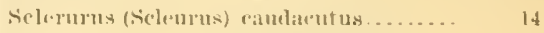

fnal entalensis..... 20

lawromed ..........

mexictums ........

Sieslecophagus eranocephlalus..........

firrugint.11........... 30,1 ?

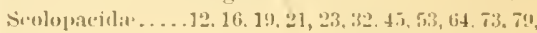
$81,80,41,861,82,991,97$

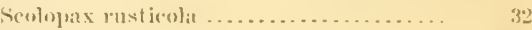

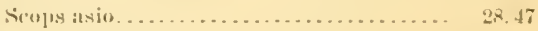

brasiliatuts . . . . . . th

cholib:1 ...................... 17

mulipe. . .................. 44

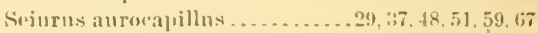

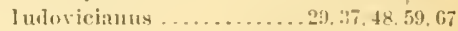
motineilla ................ 82,91

nat vi11, .............. $76,78,82,89,91,96$ movelurivensis .........14. 29.37, th 51.67

Sielasphorus tlammuliı............. 412 scintilla............... t2

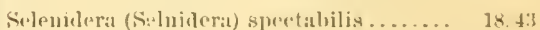

Siriuts entulria .................... 49

firpephagat griseit . ................. 55.104

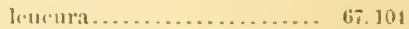

Setopluaga aurantiakea ................ is miniatu.................. 59.6n piet:1................. 59.68 ruticillia ......14, 2.-2, 24, 29, 37, tr, fix. 76 .

$7 R, 80.82,85,87,89,91,96$

torq̨uatit ....................

Sialia mexict11il . . . . . . . . . . . . . . . . 59

sialis ........................ 48,100

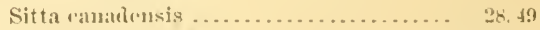

carolime

Sittace maceao ..................... 43

militaris ................ $43,6 \%$

Sittasomms sylvioiles............... 39

Siurus, sere Sciurus.

Sixlen mo species of Trxis birds ....... ix

smitlasnian Institution .......... $5,24,25,53.78$,

so. $+2.85, \pi \%, 89$

Sueorro Islamel ......54, 55, 58, 59, 60, 62, 63, 6.5. 60, 10 :

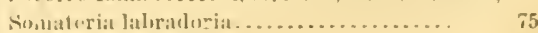

molliquima .................

spretipbilis ...............

sombrero Islind .................2.2, 101, 105

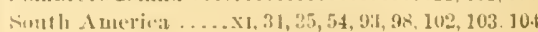


l'iles:

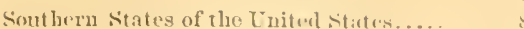

Spatula clyjuata..................... 9.53.65

Spentyto :matura..................., 91, 104 ("unicnlaria hy'pegara ........... 63,71

Spermoplita albigularis.

3

atriceps ................. 35,104

aurila................ 16

barliventris ............... 91104

rinceren . . .

rollinis ................. 24, 104

corvin: ................. 29, 3!

fortipes............... 24, 104

mutturalis ...................

hickisii .

(6)

hollm:ınui ................

liumat:t..................

minuta .................

morelluti

parra .

selistacea

semicullaris

tormpurolat

21,104

$3 !$

1,4

16

39

94. 114

21. 104

21, 104

$1 ; 0$

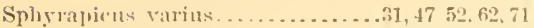

spizacitus mamluyti............... 72

m(1)

muatus ................... 4

1 vramums................. 13,4

Spizella at rigulari\$................. .

monticolit ................... 330.49

pnsilla ..................... 30. 49

surialis................... 9, 30, 4!, 60,

sorialis arizone.............. $\$ 99$

sporadimus loraci................... 75. 104

sjorophilit motelloli ................. 99

morelleti sharpei........... 99. 111

Siquatarola helvetica..........32, 53, 64, 73, 81,92

Staten Islint1, X..Y.............. 28-34

Strlyiloptrix tulvigul:t.............. 37

fulvipemis........... 51

Strnopsis allic:audla ................. bfi, 104

maculiraulus.............. 17. 106

Struorarins buthi................... 34

eaturracter............... 1, fi

сер)

parasitious ............... 6. 6.34

pomarims..............

pematorlinus............ in

Sterna :cutlaviulit ................. 7.53

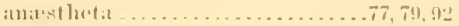

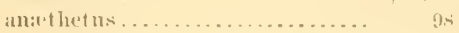

antillarum............23, 3.1. 74, 77, 79, 92

ar:anea...................

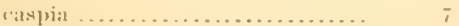

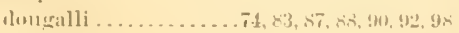

legrms ........................ ;

torsteri .................. $3,7,31,6 \mathrm{tit}, 74$

fremilia . . . F...............

fuliginusa .......... galleriv'ulat'n .................. titi

liar.llii.....................

himınlı.................. 34.95

mak'rur:ı ......................

mixima ............66, 82, 84, 86, 910, 92..98

furalisua.................. 7.34 liage:

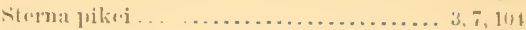

regial ..........................

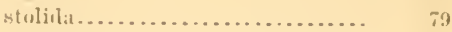

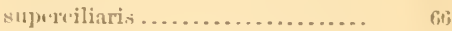

tmulıaii ...................... $\quad 7,34$

wilkmi ....................... $\quad 7$

s]). ?....................... 8, 83

Sternidir.........................

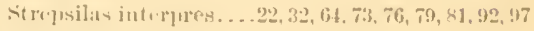

Strupilisla .....................

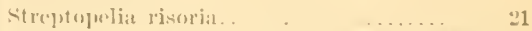

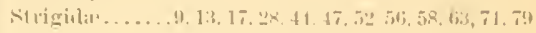

\$1, $\approx 2, \times 6,9 \%$

Strix flamuma nigreser.ns. .76,79,81,810,91, $93,97,114$ tlamme: pratincola............. (i:3. 72

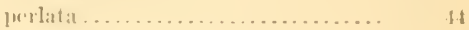

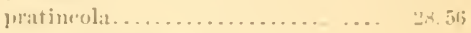

St robilıplagat + nule bator............. ;

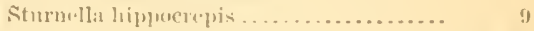
luitoviriamat .................. mitenit .....................30, 19.100 manna mexic:an:............ 69 magna meglecta.............. mw. xirana ...................

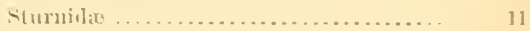

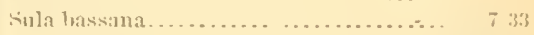

ryanops..........................

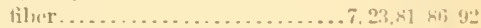

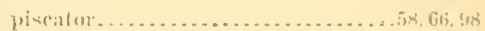
sula ....................................

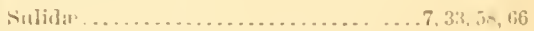

Surnia nlula ...................... gs

switt ......................... 76, .n, !1, 93

Syibrex.yeln- dominieus.............. fit; it

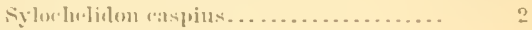

Nylvia antummalis .................

Sylviøla ju+nilis ..................

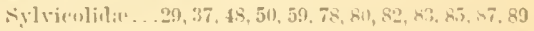

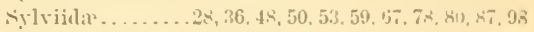

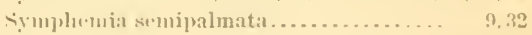

$53,64.73 .43 .81 .9297$

Synallaxis (Nyanallaxis) brumedomalalis 14

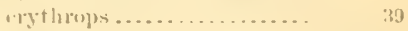

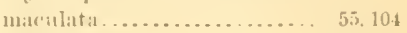

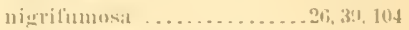
rutigrnis. ................ 331,104

syrumm liutatum ................. 17. 104

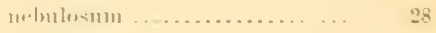

[nerspicillatum............... 9. 44

rirgatum ................... 1:3 41 6i3

T.

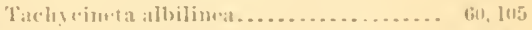
biowhr............... 650, 99

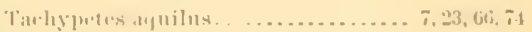

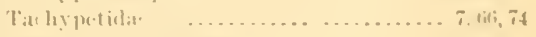

Tat.h, assinii. . . . ........ 12 3s.1144 delatirii ................ 15 ;

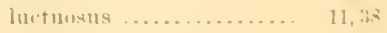

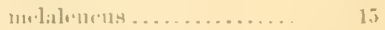

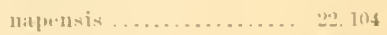
propinguus. .......... 36. is 114 rubrifrums............. tibialis ..............2.2. 35. 104

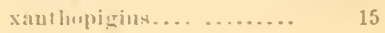


Tauagrat abloas................ Pagre. ímil . . di:(4) 21. 26.38 melameptreril

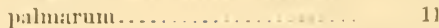

Tantagrid:1............. 15, 21, 22, 23, 26, 30, 38 $48,51,53,57,60,66,68,78,80,85,87,89,96$

Tuntaliclat ................ 16. 19, 32,45, 61, 73

Tantalus locnlator................ 16, 64, 73

Trin .............................. $7 \pi, 79$

Tetragome is frant $\%$ ii................. 4 .3

Trtranislit ..................... 12, 31, 49, 75, 8:3

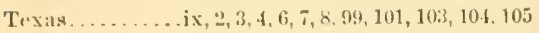
Thilassens cintiatus ...............

cispins ...................

maximus....................

renitus. . .

Thallatswdromat fregetta

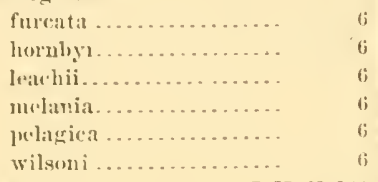

Thahurania luciat . . . . . . . . . . . . . 17,57, 6i2. 104

velunst: ................. 10, 42

wagleri ................. $76,78,91$

Thammistats anabitimus .............

Thannophilus allinis ...............2(i, 40, 51

allimulatis ........... 53

amiazonicus . . . . . . . . 14 .

bridgesi .............. 40

doliatus ............. 11.40

hollindi ............... 26,104

lezropyins.............. 34,104

melanorissus .......... 40

nevius ............... 40

nimrescens.............. 35, 104

nigricristutus ......... $2 \bar{\imath}, 104$

punctatus............. \$ $\$$

temuifaseintus ........... 35,104

transandeanus .......... 11

virgatus............. $\$ 6,104$

Thammatias candislus.................

chionurus ..................

Ineia.

virilicauslus

3.5. $10 \mathrm{~J}$

36,104

35,104

Thrasä̈tus haruyia ................. 17.72

Thripadects virgatieeps............. 52, 104

Thrush ...................... 76,78

Thryophilus eastaneum .............. 36,105

molestus .................

pleurostictus ..............

ruf:ulluts...

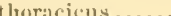

Thryothorus allimmbit ..............

bewiekii leneograster ...... 59,67 hrmunets............. 25, 104 r.tstanens .................14, 104. 105

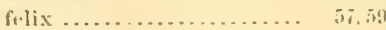
felix lawneii ........... $x$ gall)rathii ............... 14, 104 trroualdusis.........7\%, 85, 91, 96, 101 leueotis ................. 14 longirostris 'lhryothorus lutoviciams........... 29.

matulipetus ............

martinicensis ............ $8 \mathbf{7 , 9 1}$

modistus............... 20

misicus.............77, 40, 91, 104

nigrivipillus............

pleurostietus ........... 67

rufallus .............. 20.24

ruft:stens ........75, 76, 78,89,91, 104 rutilus.................. 14

sinaloa................ 59

superciliaris ............. 53, 101

T'igrisoma brasilie.use............... 12 cabinisi ...................46, 65. 73

tigrinil ..................... 21

Tinamidice.......................... 16, 19, 64

Tinanus frantzii..................... t5. 104

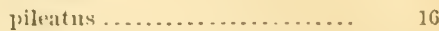

robustus ................... 19,45

Timnunitulus sparverius . . . .22, 44, 47, 52, 56, 63, 72 sparverius antilharum ..76, 79,82,84,

$86,88,90,92$

sparverius caribhacarum.... 97 sparreroides............ 8.22

Tityra alhiturutu's .................. 11, 26, 41 jersonatal ...............11, 41,51, 62, 70

'Tobago, Island of . ................... 99, 101

Todirostrum • inereun................11, 26, 40 ecaurlatum............. 40 megacephalum........... 15 nigrieeps................ 15,40 olivaceum . ............17, 104, 105 plumbenm .............. $5 f$ schistacriceps........... 18,70 supercilintis............ 55,104

Totanus flavipes................... 98 melanolencus................ 9.9

Totipalmi ........................ 7

Toxostomal curvirostris ................ lecontei .................. 3, 104, 105

Tres Marias Islands...... x, 17, 35, 54, 56, 57,59, 60, $61,62,63,64,65,66,101,103,104$

Tringa canutus .................... 32 nacrulata ...................90,02,03 minutilla...................81, 92,93

wilsonit .................... 10

Tringoicles.....16, 32, 45, $50,64,73,77,79.81,80,88$, 92,95

Trinflat, Island of .......... x, 46,58, 103, 104 Trochilidia......10, 14, 17, 20, 22, 25. 27, 31, 35, 42, $47,52,54,57,62,71,75,78,80,82,-4,85,88,89,96$ Trothilıs aquila .................... aurigularis................. 17,104 colubris..................31, 42, 47,71

Troglodytus aïlon ................... 29,49 americaums.............. 29 brachyurus.............. 9k, 104 hyemalis ................. 29,49

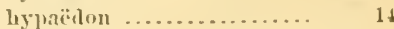
inqquietus ............... 24, 36 insularis................54, 5s, 59 internedius .............. 36,50 tobagensis............. 99, 104

Troglodytidia ......10, 14. 18, 20. 25, 29, 36, 49, 50,53, $55,57,58,59,67,7 \pi, 80,85,87,89,96,98$ 
Pagre. 56,62

aurantiventris .............. 10.42

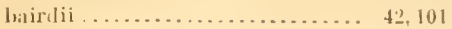

bracc"itlts ...................

caligatux .................... 10,42

(ritre(ulus .................. 62.70

clathritus .................... 42

(oncimun................. 17,42, 104

(ximius.......................... 55. 104

matcrourat ................... I0

massem .....................10. 26, 42

molanocephalı. .............. 20

purlla ..................... $4 ., 70$

toncllus...................... 20, 26, 12

viriclis ....................... 10

Trogonili: $\ldots \ldots \ldots \ldots \ldots \ldots 10,17,20,26,42,56,62,70$

Tryugites lufieserens................ 32

Turdid: $x^{2} \ldots \ldots .15,16,18,20,2 x, 36,48,49,50,53,56,54$ $59,67,78,80,82,83,85,87,89,92,93,46$

Turdus aliura. . . . . . . .

lirumnelus .................. 77,104

‘aribhatus ............. 77. 85,90, 104

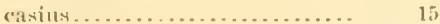

Havirostris................56, 59,67

fusctisen. . . . . . . . . . . .

grayi …............24, 36, 50,56,59,67

grmuopthalmus .............. 96

hi:11. welli ................... 54, 104

lenchauchen ................. 36

migraturins................ $2 x, 48$

minimus.................

mustrliums.................28, 48,67

nevius .................. 3,28

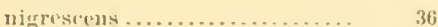

nigrinostris ..........7\%, $8(1, x .5,90,96,104$

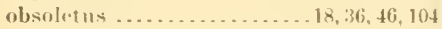

pallawi ..................... 28,48

plebius..................... 36

reevei ...................... 53, 104

solitariny .................. 4

swainsonii $\ldots \ldots \ldots \ldots \ldots \ldots . . \ldots 24,36,48,67$ ustulatus ................. 56,59

sp. ? ......................... 90

Tympanuelus amerieanus ............ 99

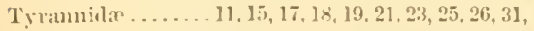
$35,40,46,47,51,53,55,57,61,66,70,78$,

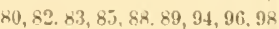

Tyraniscus parrus.............. 17, 18,40,104 villisimus.

'I'yramula ciurascens................ 3, 4. 105 thariventris ................. 15

Tyramnulus brumnejeapillus........17, 18,40, 105 s.mitlavus.............. 26

Tyrumus carolinensis ............26, 31,47, 70 castinii .................. 2,105

crissiostris................ 62.70

gris.us.................. 22, 26

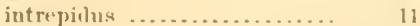

melan.holicus .......11, $41,53,85,91,06$

melanchulicus enouchii ........ 61

m.litucholicus satrapa....... 61,70

rostratus $\ldots 76,78, \times 0, \times 2, \times 3,85,25,91,96$

satrajpit ................... 26,51

tyramus ................... $1(80)$

vertiralis ................

vocitirans...............
U.

I'agre.

Tnited States, Atlantie coast of. ........ 6

United Statı.s National Musemun........ 67

Bul|,tin of .....................

l'recellings of . ...................

$80, x^{2}, x_{i}, 87,89,90,93,94,96,49$

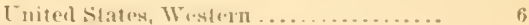

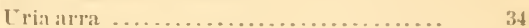

grylle........................... 34

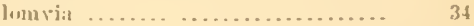

Erinator parilieus................... 105

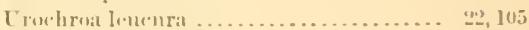
pile:ta .................. 11

Tresl jettruticrimsa .................... 22, 105

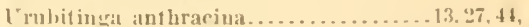
$54,15,72,81,91$

minincia ............... 54

yommra ................13, 44. 52, 63, 72

Tramania torla ..................

\section{1.}

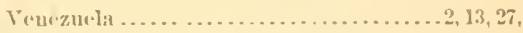
$34,35,54,55,75,101,102,103$

Viruo atricapillus................... 60.

b.lli........................... Gs

rirmioli......................... 37

flavifims . . . . . . . . . . . . . . . $14,29,6 \sigma^{\circ}$

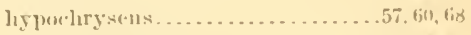

noveluracensis.................2!). 4*, ti8

ochracelus..................... tio

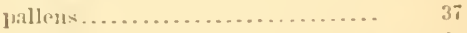

plumble-11. ....................

soliturius .....................2n, 18,60

Vireolanius pulchellus............... $18,37,09$

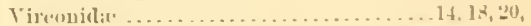

$22,25,29,37,45,51.57 .60,08,78,80,80,85,57,82,96$

Vireosylvia altilompa............... 9, 22

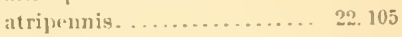
lugutensis. ................ 20

caliulris .................. \&2.91

cilidris dominicana. .......76, $7 . . . \times 0$, (i) $\times 7,89,91,90,105$ tlavovirulis.............. $14,37.60$, the gil rat .................... 29, 4x gilvil swainsuni ............ o. josepha ................... 37 miluster................ 55 olivareal .................29,3i,48 philiulelphia ............ 37

Tolatinia jacarina......... 16i. 24 394 51, 600, 69, 90

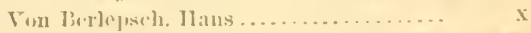

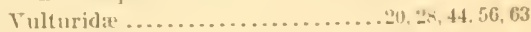

W.

Wirlbler, Bachman's............... 95,99

Law rencris ..............

Swainson's ..............

Wishingtom Territury.............. $7, x$

W'.lls, dohn Grant ..................

Wistern (I'...) livers...............

Wrell ..........................

Liw 
X.

Sintloceplonlus ietorer.phlıalus. spurius ....

Xanthentra (Xinthura) guatlonalensis.... luxuแsia..................

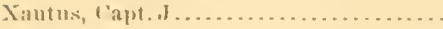

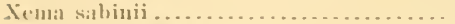

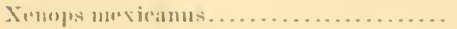

Xiphurhynelus lafresnayamo.........

I.

Yucitin 50--5i3, 54, 55, 93, 94, 95, 98, !(1)1, 102, 103,105

(i)

2,105

69

61

Z.

Page.

39

59

7. 34

14. 39

10

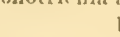

Zoological horety of Loment, l'rocondingy

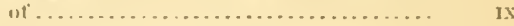







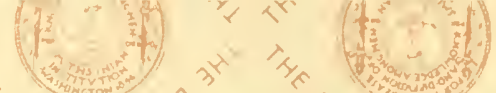

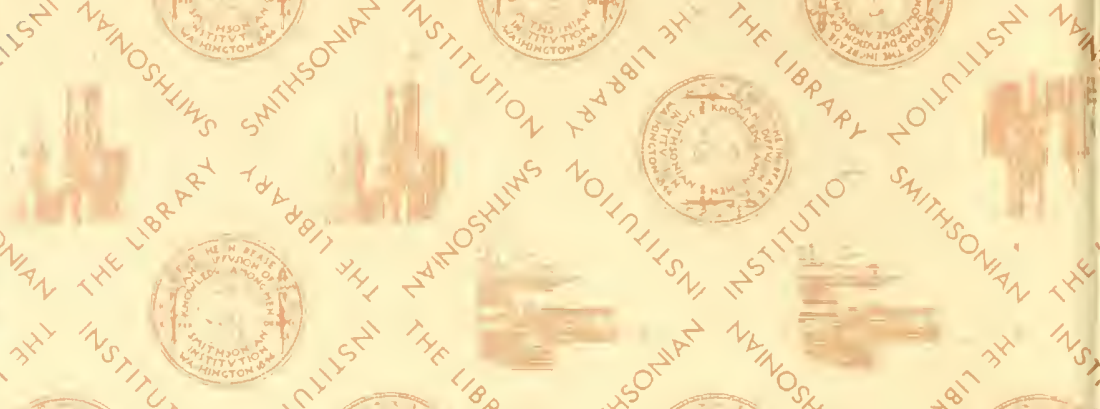

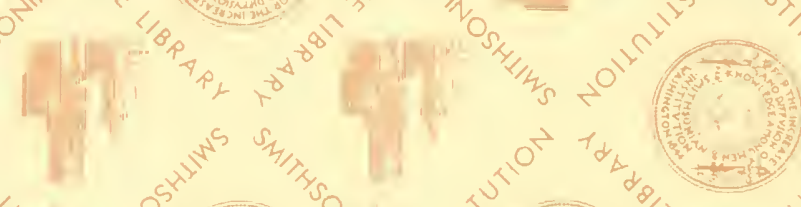

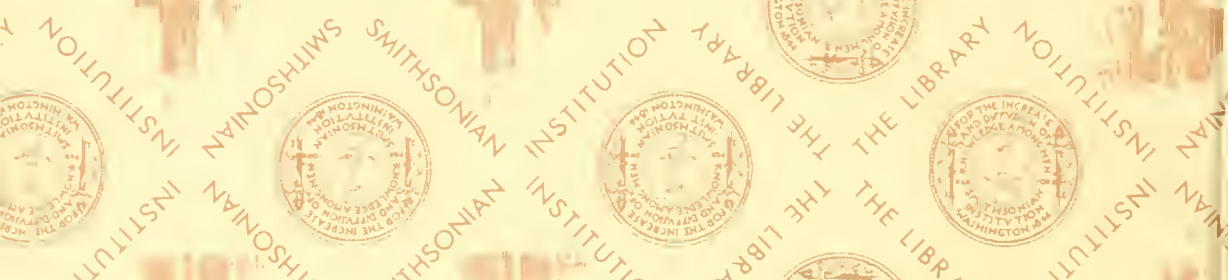

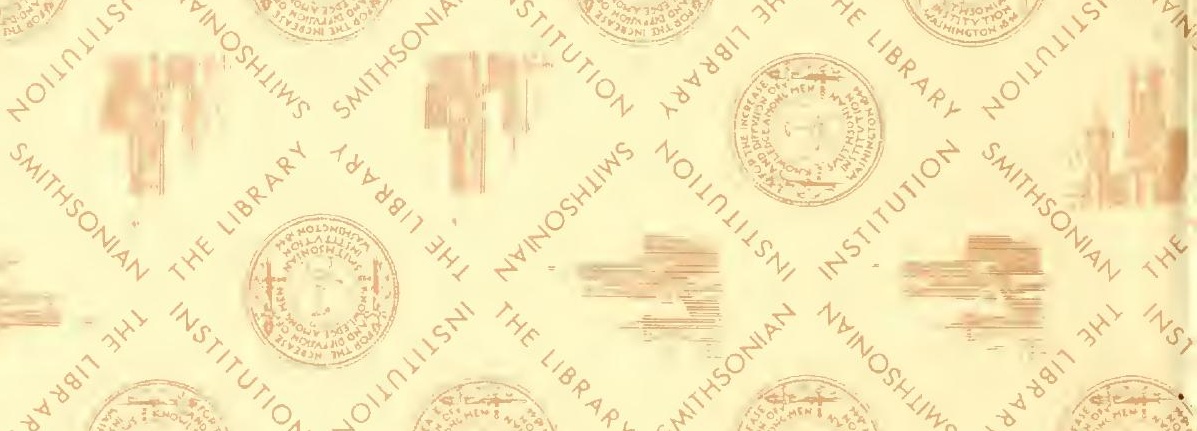

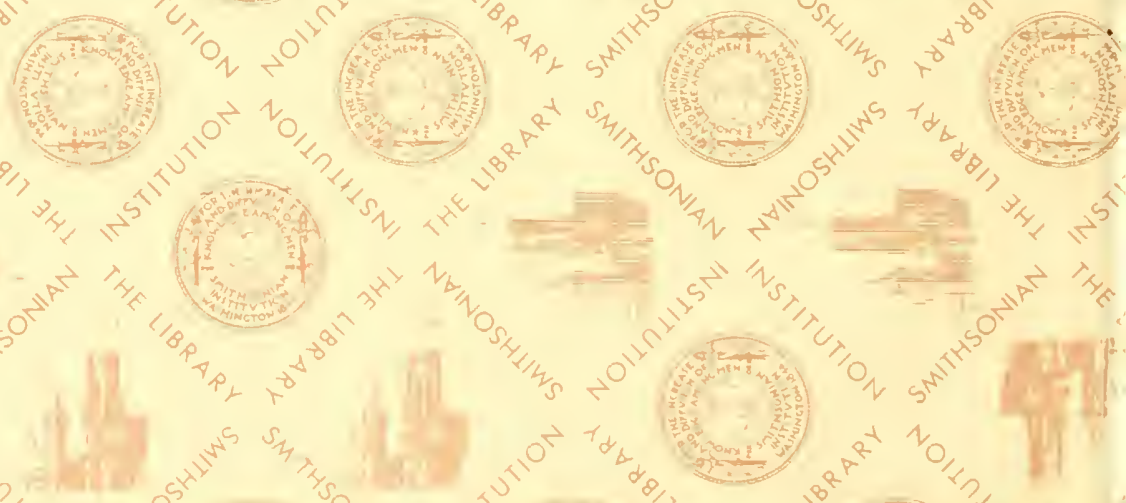

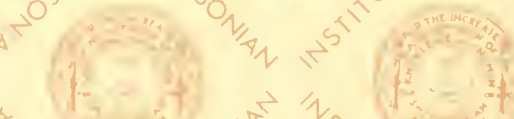


